\title{
Design and implementation of event-based multi-rate controllers for networked control systems
}

September 2020

Author: José Joaquín Alcaina Acosta

Director: Dr. Ángel Miguel Cuenca Lacruz 



\section{Preface}

Networked control system (NCS) is a relatively new technique with obvious advantages compared to conventional local control, such as reduced wiring and consequently, a decrease in the space and weight occupied by these systems. This leads to an increased battery autonomy, more robustness to changes due to the modularization of the systems and, in some cases, the control centralization of a range of different elements in a single point. There is also the possibility of using wireless connections to control systems located at long distances, which would be otherwise inaccessible through wiring. These advantages make NCS extremely attractive in a number of fields such as robotics, avionics, the medical and chemical fields, or simply in systems requiring an optimization of costs, maintenance or space.

Nevertheless, the presence of a network in a feedback loop adds analysis and design problems to a control system, which do not occur in conventional control systems, such as delays, packet disorders, packet dropouts, limitation of bandwidth imposed by the network, etc. This thesis will address the problems occurring in networked-based control and will try to reduce network usage, trying to respect the system's ideal performance as much as possible.

The current thesis is divided into three parts.

- The first part, composed by paper 1, addresses event-triggered control to minimize the bandwidth consumed in NCS. Specifically, it approaches a particular case of periodic event-triggered control (PETC) and depicts a simulation method to obtain its optimal parameters for the system in which it is used.

- Papers 2, 4, 6 describe packet-based control and multirate control, that address NCS problems such as network delays, packet dropouts and packet disorders, and the scarce data due to slow sensor usage in order to save battery in sensing tasks and transmissions of the sensed data. Also addressed, is how despite the existence of measurement noise and disturbances, time-varying dual-rate Kalman filter based prediction techniques observe the complete state of the system. paper 7 tackles a non-linear model that uses all the previous solutions together with an extended Kalman filter to present another type of structure for an autonomous vehicle that, due to future information obtained through these techniques, can remotely carry out high level tasks, such as decision making and monitoring of variables. 
- The third part consists of papers 3 and 5 , presenting a method describing how to obtain and analyse SISO dual-rate systems frequency response. By using QFT (quantitative feedback theory) procedures its behavior is studied when faced with specific uncertainties or problems in the network.

\begin{abstract}
Aim of thesis
This thesis attempts to solve some of the most common problems that exist in NCS, such as time-varying delays, packet disorders, packet dropouts and bandwidth limitation. Other frequent problems are scarce computational and energy resources of the local system devices. In order to deal with these problems, the integration of multirate control, packet-based control, predictorbased control and event-based control techniques has been proposed.
\end{abstract}

\title{
Thesis outline and publication history
}

The structure of the thesis is as follows: chapter 1 is an introduction presenting concepts and particularities of networked control systems and the different mechanisms used when faced with these particularities. chapter 2 to chapter 8 are the papers published about the works developed throughout this doctorate programme. chapter 9 closes this thesis presenting conclusions and ideas for future works.

\section{Paper 1. Control of the rotary inverted pendulum through threshold-based communication}

This paper deals with the real implementation of a periodic event-triggered control in a Networked Control System. The bandwidth used by the control loop in the shared communication medium is reduced by using a thresholdbased communication. The paper explains an easy procedure to determine by simulation the optimal values of these thresholds in order to minimize a cost index.

The paper has been published as: 
V. Casanova, J. Alcaina, J. Salt, R. Pizá, Á. Cuenca. "Control of the rotary inverted pendulum through threshold-based communication", ISA Transactions 62, 357-366, 2016, DOI: https: //doi. org/10.1016/j. isatra. 2016.01.009

\section{Paper 2. A packet-based dual-rate PID control strategy for a slow-rate sensing networked control system}

This paper introduces a delay-dependent packet-based dual-rate control strategy to face time-varying network-induced delays, packet dropouts and packet disorder in a Networked Control System. Slow-rate sensing enables to achieve energy saving and to avoid packet disorder. Fast-rate actuation makes reaching the desired control performance possible. The proposed control solution is able to approximately reach the nominal (no-delay, no-dropout) performance despite the existence of time-varying delays and packet dropouts. Control system stability is ensured in terms of probabilistic Linear Matrix Inequalities (LMIs). Via real-time control for a Cartesian robot, results clearly reveal the superiority of the control solution compared to a previous proposal by the authors.

The paper has been published as:

Á. Cuenca, J. Alcaina, J. Salt, V. Casanova, R. Pizá. "A packet-based dual-rate PID control strategy for a slow-rate sensing networked control system", ISA Transactions 76, 155-166, 2018, DOI: https://doi.org/10.1016/j. isatra. 2018. 02.022

\section{Paper 3. Dual-rate sampled-data systems. Some interesting consequences from its frequency response analysis}

The paper introduces a new procedure in order to properly analyze SISO dualrate systems (DRS) and provides straightforward answers to some common general questions about this kind of systems. Frequency response analysis based on DRS lifting modeling can lead to interesting results about stability margins or performance prediction. As a novelty, it is explained how to understand DRS frequency response and how to handle it for an easy computation of magnitude and phase margins keeping classical frequency domain methods. 
The paper has been published as:

J. Salt, J. Alcaina, "Dual-Rate Sampled-Data Systems Frequency Response: Interpretation and Some interesting consequences", International Journal of General Systems 48(5), 554574, 2019, DOI: https: //doi. org/10. 1080/03081079. 2019. 1608984

\section{Paper 4. Delay-independent dual-rate PID controller for a packet-based networked control system}

In this paper, a novel delay-independent packet-based dual-rate control structure for a networked control system is proposed. This work is a better variation of the solution presented in paper 2. As a delay-independent control solution, no network-induced delay measurement is needed for controller implementation. In addition, the control scheme is applicable to open-loop unstable plants. As in paper 2, control system stability is ensured in terms of LMI and simulation results are experimentally validated.

The paper has been published as:

J. Alcaina, Á. Cuenca, J. Salt, V. Casanova, R. Pizá, "Delayindependent dual-rate PID controller for a packet-based networked control system", Information Sciences 484, 27-43, 2019, DOI: https: // doi.org/10.1016/j. ins. 2019.01.059

\section{Paper 5. Multirate control strategies for avoiding sample losses. Application to UGV path tracking}

The objective of this contribution is to analyze and compare dual-rate inferential control and model-based dual-rate control trying to assess their behavior under different perspectives. The algorithm explained in paper 3 is used for computing a MR system frequency response for these control structures. The robust performance and disturbance effects are studied in detail under sample losses and process uncertainty and some considerations are reported. A new QFT (quantitative feedback theory) procedure for dual-rate systems analysis 
is also described. Analysis and simulation examples and experimental results are introduced.

The paper has been published as:

J. Salt, J. Alcaina, Á. Cuenca, A. Baños, "Multirate Control Strategies for avoiding samples losses. Application to UGV path tracking", ISA Transactions 101, 130-146, 2020, DOI: https: // doi.org/10.1016/j. isatra.2020.01.025

\section{Paper 6. Energy-efficient control for an unmanned ground vehicle in a wireless sensor network}

In this paper, an energy-efficient control solution for an Unmanned Ground Vehicle in a Wireless Sensor Network is proposed. This novel control approach integrates solutions from papers 1,2 and 4 and a time-varying dual-rate Kalman filter. The systematic combination of these control techniques allows the UGV to track the desired path preserving performance properties, despite: i) existing scarce data due to the reduced usage of the wireless sensor device, which results in less number of transmissions through the network, and hence, bandwidth and battery saving, ii) appearing some wireless communication problems such as time-varying delays, packet dropouts and packet disorder, and iii) coping with a realistic scenario where external disturbance and sensor noise can arise. The main benefits of the control solution are illustrated via simulation.

The paper has been published as:

J. Alcaina, Á. Cuenca, J. Salt, M. Zheng, M. Tomizuka, "Energy-efficient Control for an Unmanned Ground Vehicle in a Wireless Sensor Network", Journal of Sensors, Vol. 2019, ID. 7085915, 16 pages, 2019, DOI: https: // doi. org/10. $1155 / 2019 / 7085915$ 


\section{Paper 7. A remote control strategy for an autonomous vehicle with slow sensor using Kalman filtering and dual-rate control}

This work presents a novel remote control solution for an Autonomous Vehicle, where the system structure is split into two sides through a communication network, which introduces time-varying delays and packet disorder. An Extended Kalman Filter is used to cope with the non-linearities that appear in the global model of the AV. The EKF fuses the data provided by the sensing devices of the AV in order to estimate the AV state, reducing the noise effect. Additionally, the EKF includes an $h$-step-ahead state prediction stage, which, together with the consideration of a packet-based control strategy, enables facing the network-induced delays. Since the AV position is provided by a camera, which is a slow sensing device, a dual-rate controller is required to achieve certain desired (nominal) dynamic control performance. The use of a dual-rate control framework additionally enables saving network bandwidth and deals with packet disorder.

The paper has been published as:

A. Cuenca, W. Zhan, J. Salt, J. Alcaina, C. Tang, M. Tomizuka, "A Remote Control Strategy for an Autonomous Vehicle with Slow Sensor using Kalman Filtering and Dual-rate Control", Sensors 19(13), 2983, 2019, DOI: https://doi. org/10.3390/s19132983

\section{Acknowledgements}

Cabe destacar que, desde que terminó mi contrato predoctoral con la universidad hasta el momento en que termino este libro debe haber alrededor de cien mails en mi bandeja de correo diciéndome, en resumidas cuentas, "Venga vago, termina de una vez de escribir la tesis". Por lo tanto, veo necesario, en primer lugar, dar mi más sincero agradecimiento al director de esta tesis, Ángel Cuenca. Sin la ayuda que me ha prestado durante todo el proceso de realización de la misma me hubiese sido imposible elaborarla; sin su meticulosidad y rigurosidad seguro que los resultados que hemos conseguido publicar hubiesen sido de mucha peor calidad y sin su apoyo y ánimos seguro que este libro no se hubiese terminado de escribir. 
De la misma manera, gran parte del mérito de esta tesis se lo debo al resto de mi grupo de investigación. Sin esperar nada a cambio me han acogido en su grupo y han dedicado todo el tiempo que he necesitado en ayudarme en lo que fuera. Julián, Vicente y Ricardo, mi más sincero agradecimiento por vuestro trato tanto profesional como personal.

Agradezco a todos los coautores con los que he conseguido publicar. Son responsables de que la contribución de esta tesis sea de muchísima mejor calidad que si no hubiese trabajado con ellos.

A mi señora, Elisabet, le debo que esta tesis esté escrita en el idioma de Shakespeare y también el de su madre (la madre de Elisabet, aunque también la de Shakespeare). Ella ha tenido la capacidad de aguantarme durante estos años, cosa que también tiene su mérito. Además me ha elegido, hasta que se demuestre lo contrario, como el padre de su hijo, Joan, la persona que, junto a ella, más quiero en el mundo.

Por último, agradezco al resto de mi familia su apoyo incondicional.

\section{Financial support}

- This thesis was funded by "Contratos predoctorales del programa para la Formación de Personal Investigador (FPI) de la UPV 2015 - Subprograma $1 "$.

- This research was partially funded by Grant RTI2018-096590-B-100 from the Spanish government, and by the European Commission as part of Project H2020-SEC-2016-2017 - Topic: SEC-20-BES-2016 - Id: 740736 - "C2 Advanced Multi-domain Environment and Live Observation Technologies" (CAMELOT). Part WP5 supported by Tekever ASDS, Thales Research and Technology, Viasat Antenna Systems, Universitat Politècnica de València, Fundação da Faculdade de Ciências da Universidade de Lisboa, Ministério da Defensa Nacional - Marinha Portuguesa, Ministério da Administração Interna Guarda Nacional Republicana. 



\section{Abstract}

This thesis attempts to solve some of the most frequent issues that appear in Networked Control Systems (NCS), such as time-varying delays, packet losses and packet disorders and the bandwidth limitation. Other frequent problems are scarce computational and energy resources of the local system devices. Thus, it is proposed to integrate multirate control, packet-based control, predictor-based control and event-based control techniques. The control designs have been simulated using Matlab-Simulink and Truetime, the stability has been analysed by LMIs and QFT, and the experimental validation has been done on an inverted pendulum, a 3D cartesian robot and in low-cost mobile robots.

Paper 1 addresses event-based control, which minimizes the bandwidth consumed in NCS through a periodic event-triggered control and presents a method to obtain the optimal parameters for the specific system used.

Papers 2, 4 and 6 include packet-based control and multirate control, addressing problems such as network delays, packet dropouts and packet disorders, and the scarce data due to low sensor usage in order to save battery in sensing tasks and transmissions of the sensed data. Also addressed, is how despite the existence of measurement noise and disturbances, time-varying dual-rate Kalman filter based prediction techniques observe the complete state of the system. Paper 7 tackles a non-linear model that uses all the previous solutions together with an extended Kalman filter to present another type of structure for an autonomous vehicle that, due to future information obtained through these techniques, can remotely carry out high level tasks, such as decision making and monitoring of variables.

Papers 3 and 5, present a method for obtaining and analyzing the SISO dualrate frequency response and using QFT procedures to study its behavior when faced with specific uncertainties or network problems. 



\section{Resumen}

Con esta tesis se pretende dar solución a algunos de los problemas más habituales que aparecen en los Sistemas de control basados en red (NCS) como son los retardos variables en el tiempo, las pérdidas y el desorden de paquetes, y la restricción de ancho de banda y de recursos computacionales y energéticos de los dispositivos que forman parte del sistema de control. Para ello se ha planteado la integración de técnicas de control multifrecuencial, de control basado en paquetes, de control basado en predictor y de control basado en eventos. Los diseños de control realizados se han simulado utilizando MatlabSimulink y Truetime, se ha analizado su estabilidad mediante LMIs y QFT, y se han validado experimentalmente en un péndulo invertido, un robot cartesiano $3 \mathrm{D}$ y en robots móviles de bajo coste.

El artículo 1 aborda el control basado en eventos, el cual minimiza el ancho de banda consumido en el NCS mediante un control basado en eventos periódicos y presenta un método para obtener sus parámetros óptimos para el sistema específico en que se utilice.

Los artículos 2, 4 y 6 añaden el control basado en paquetes, así como el control multifrecuencia, que aborda problemas de falta de datos por bajo uso del sensor y los retardos, pérdidas y desórdenes de paquetes en la red. También afrontan, mediante tecnicas de predicción basadas en un filtro de Kalman multifrecuencia variable en el tiempo, los problemas de ruido y perturbaciones, así como la observación de los estados completos del sistema. El artículo 7 hace frente a un modelo no lineal que utiliza las anteriores soluciones junto con un filtro de Kalman extendido para presentar otro tipo de estructura para un vehículo autónomo que, gracias a la información futura obtenida mediante estas técnicas, puede realizar de forma remota tareas de alto nivel como es la toma de decisiones y la monitorización de variables.

Los artículos 3 y 5 , presentan una forma de obtener y analizar la respuesta en frecuencia de sistemas SISO multifrecuencia y estudian su comportamiento ante ciertas incertidumbres o problemas en la red haciendo uso de procedimientos QFT. 



\section{Resum}

Amb aquesta tesi es pretén donar solució a alguns dels problemes més habituals que apareixen als Sistemes de Control Basats en xarxa (NCS) com son els retards d'accés i transferència variables en el temps, les pèrdues y desordenament de paquets, i la restricció d'ampli de banda així com de recursos computacionals i energètics dels dispositius que foment part del sistema de control. Per tal de resoldre'ls s'ha plantejat la integració de tècniques de control multifreqüencial, de control basat en paquets, de control basat en predictor i de control basat en events. Els dissenys de control realitzats s'han simulat fent ús de Matlab-Simulink i de TrueTime, s'ha analitzat la seua estabilitat mitjançant LMIs i QFT, i s'han validat experimentalment en un pèndul invertit, un robot cartesià 3D i en robots mòbils de baix cost.

L'article 1 aborda el control basat en events, el qual minimitza l'ampli de banda consumit a l'NCS mitjançant un control basat en events periòdics i presenta un mètode per a obtindré els seus paràmetres òptims per al sistema específic en el qual s'utilitza.

Els articles 2, 4 i 6 afegeixen el control basat en paquets, així com el control multifreqüència, que aborda problemes de falta de dades per el baix us del sensor i els retards, pèrdues i desordre de paquets en la xarxa. També afronten, mitjançant tècniques de predicció basades en un filtre de Kalman multifreqüència variable en el temps. Els problemes de soroll i pertorbacions, així com la observació dels estats complets del sistema. L'article 7 fa referència a un model no lineal que utilitza les anteriors solucions junt a un filtre de Kalman estès per a presentar altre tipus d'estructura per a un vehicle autònom que, gracies a la informació futura obtinguda mitjançant aquestes tècniques, pot realitzar de manera remota tasques d'alt nivell com son la presa de decisions i la monitorització de variables.

Els articles 3 y 5 presenten la manera d'obtindre i analitzar la resposta en frequencia de sistemes SISO multifreqüència i estudien el seu comportament front a certes incerteses o problemes en la xarxa fent us de procediments QFT. 



\section{Contents}

Preface $\ldots \ldots \ldots \ldots \ldots \ldots \ldots \ldots \ldots \ldots \ldots \ldots \ldots \ldots \ldots$ ii

Aim of thesis $\ldots \ldots \ldots \ldots \ldots \ldots \ldots \ldots \ldots \ldots \ldots$ iv

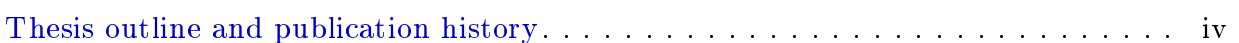

Acknowledgements. . . . . . . . . . . . . . . . . viii

Financial support $\ldots \ldots \ldots \ldots \ldots \ldots \ldots \ldots \ldots \ldots \ldots$ ix

Contents xvii

List of Figures $\quad$ xxiii

List of Tables $\quad$ xxxi

List of Acronyms $\quad$ xxxiii

1 Introduction 1

1.1 Sampling methods $\ldots \ldots \ldots \ldots \ldots \ldots \ldots \ldots \ldots \ldots$

1.2 Networked control systems. . . . . . . . . . . . . . . . . . 3

1.2 .1 Advantages . . . . . . . . . . . . . . . . . . . . 4

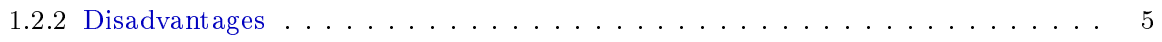

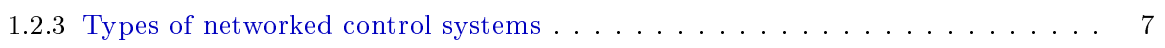

1.3 Event-triggered control . . . . . . . . . . . . . . . . . . . . . 10

1.3.1 Time Triggered Control (TTC) and Event Triggered Control (ETC) . . . . . . 10

1.3 .2 Periodic Event-Triggered Control. . . . . . . . . . . . . . . . . 11

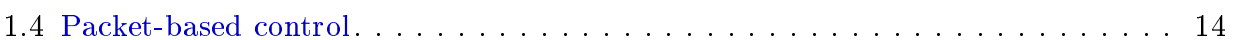

xvii 


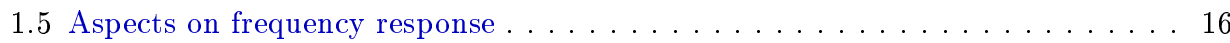

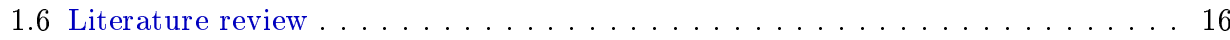

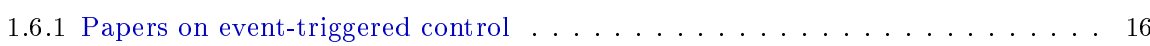

1.6.2 Papers on packet-based control . . . . . . . . . . . . . . 17

1.6.3 Papers on multi-rate frequency analysis. . . . . . . . . . . . . . 17

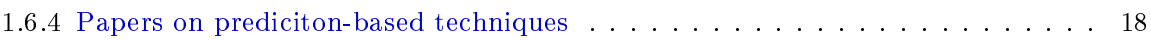

1.6.5 Papers on multi-rate control. . . . . . . . . . . . . . . . . . . . . 18

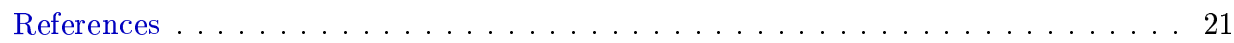

2 Paper 1. Control of the rotary inverted pendulum through thresholdbased communication

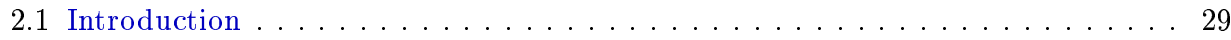

2.2 Conventional control of the rotary inverted pendulum . . . . . . . . . . 33

2.3 Threshold-based control of the rotary inverted pendulum . . . . . . . . 38

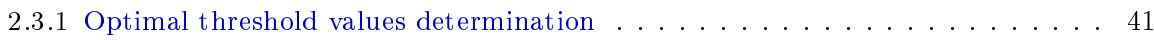

2.3.2 Experimental results with threshold-based control . . . . . . . . . . . . 46

2.4 Conclusions and future work $\ldots \ldots \ldots \ldots \ldots \ldots \ldots \ldots$

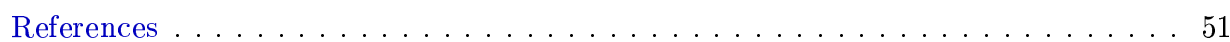

3 Paper 2. A packet-based dual-rate PID control strategy for a slow-rate sensing networked control system 55

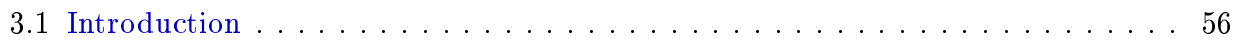

3.2 Problem description $\ldots \ldots \ldots \ldots \ldots \ldots \ldots \ldots \ldots \ldots$

3.3 Packet-based control strategy. Prediction stage . . . . . . . . . . . . . 63

3.3 .1 Slow-rate PI controller . . . . . . . . . . . . . . . . . . . . . . . 65

3.3 .2 Rate converter . . . . . . . . . . . . . . . . . . . . . . 66

3.3 .3 Fast-rate PD controller . . . . . . . . . . . . . . . . . . . . . 67

3.3 .4 Prediction Stage . . . . . . . . . . . . . . . . . . . . . . . 68

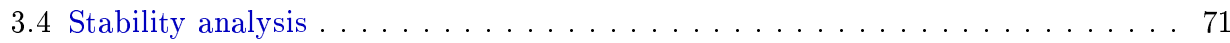

3.4 .1 Closed-loop model via lifting . . . . . . . . . . . . . . . . . . . . 71

3.4 .2 Closed-loop stability . . . . . . . . . . . . . . . . . . . . . . . 73

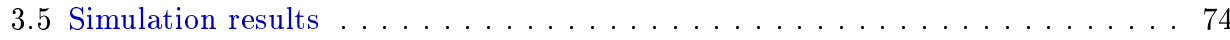

3.5.1 Simulation data . . . . . . . . . . . . . . . . . . . . . 74

xviii 
3.5.2 Data analysis via cost indexes. . . . . . . . . . . . . . . . . . 77

3.6 Experimental results. . . . . . . . . . . . . . . . . . . . . 79

3.7 Conclusions. . . . . . . . . . . . . . . . . . . . . . . 81

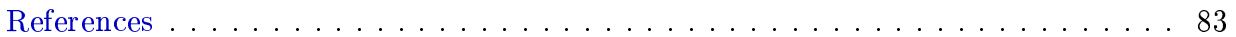

4 Paper 3. Dual-rate sampled-data systems. Some interesting con$\begin{array}{ll}\text { sequences from its frequency response analysis } & 87\end{array}$

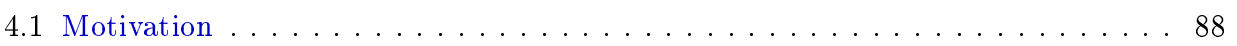

4.2 DRS frequency response computation: a classical interpretation. . . . . . . . 89

4.2 .1 Preliminaries and notation. . . . . . . . . . . . . . . . . 89

4.2 .2 Sampled-data lifted systems . . . . . . . . . . . . . . . . . . . 91

4.2 .3 DRS Frequency Response . . . . . . . . . . . . . . . . . . . . 92

4.3 Frequency Response Interpretation . . . . . . . . . . . . . . . . 93

4.4 Stability Margins for DRS . . . . . . . . . . . . . . . . . . 98

4.4 .1 Example. . . . . . . . . . . . . . . . . . 99

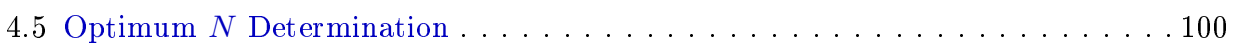

4.5.1 Example 1. . . . . . . . . . . . . . . . . . . . . . . . . . . . . 102

4.5 .2 Example 2. . . . . . . . . . . . . . . . . . 103

4.6 Conclusions. . . . . . . . . . . . . . . . . . . . . . 107

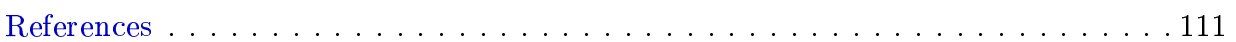

5 Paper 4. Delay-independent dual-rate PID controller for a packetbased networked control system 115

5.1 Introduction . . . . . . . . . . . . . . . . . . . 116

5.2 Problem description . . . . . . . . . . . . . . . . . . . . . 120

5.2.1 Time-varying delays, packet dropouts, and packet disorder . . . . . . . . . . 120

5.2 .2 Control structure . . . . . . . . . . . . . . . . . . . . . . . . . . . 122

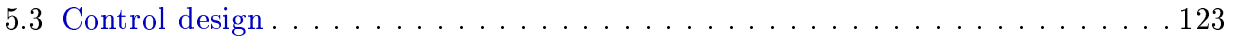

5.3 .1 Preliminaries . . . . . . . . . . . . . . . . . . . . . . . . . . . . 124

5.3 .2 Slow-rate PI controller . . . . . . . . . . . . . . . . . . . . . . . 125

5.3 .3 Rate converter . . . . . . . . . . . . . . . . . . . . . . 126

5.3 .4 Fast-rate PD controller . . . . . . . . . . . . . . . . . . . . . . . 127

5.3 .5 Prediction Stage . . . . . . . . . . . . . . . . . . . . . . . . . . . . . . 129 
5.4 Stability analysis . . . . . . . . . . . . . . . . . . 130

5.4 .1 Closed-loop model via lifting . . . . . . . . . . . . . . . . . . . 130

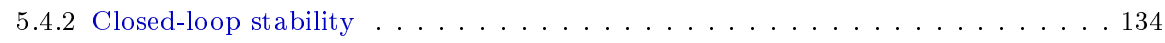

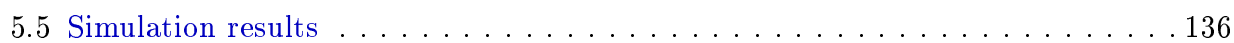

5.5.1 Simulation data. Control system stability assessment . . . . . . . . . . 136

5.5 .2 System responses. Cost indexes $J_{1}$ and $J_{2} \ldots \ldots \ldots \ldots \ldots \ldots \ldots$

5.5.3 Model mismatch. Cost indexes $J_{3}$ and $J_{4} \ldots \ldots \ldots \ldots \ldots 14 \ldots \ldots \ldots$

5.6 Experimental results. . . . . . . . . . . . . . . . . . . . . . . 144

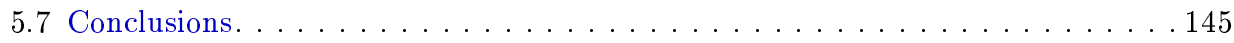

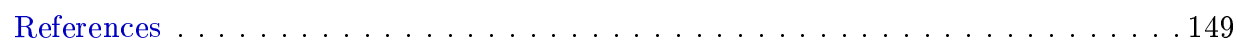

6 Paper 5. Multirate control strategies for avoiding sample losses. Application to UGV path tracking 155

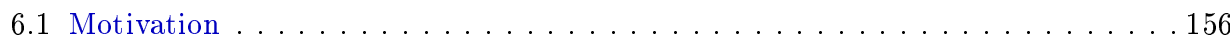

6.2 Problem Statement. . . . . . . . . . . . . . . . . . . . . . . . . . . . 159

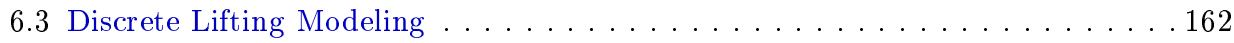

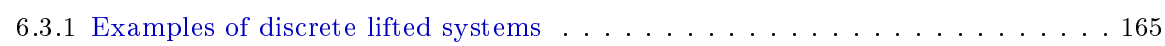

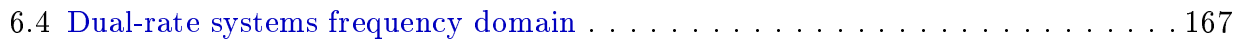

6.4.1 DR Systems Frequency Response Computation . . . . . . . . . . . . . . 167

6.4 .2 Frequency Response Interpretation . . . . . . . . . . . . . . . . . . 168

6.4.3 Dual-rate systems quantitative feedback theory . . . . . . . . . . . . . . 170

6.5 Closed-loop lifted models. . . . . . . . . . . . . . . . . . . 171

6.5 .1 Example. . . . . . . . . . . . . . . . . . . . . . . . . . 174

6.6 Simulations . . . . . . . . . . . . . . . . . . . . . . . . . . 180

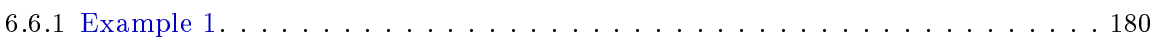

6.6 .2 Example 2. . . . . . . . . . . . . . . . . . . . . . . . 184

6.7 Experimental Application . . . . . . . . . . . . . . . . . . . . 188

6.7 .1 Path tracking. . . . . . . . . . . . . . . . . . . . . . . . . . . . 189

6.7 .2 Dual-rate controller . . . . . . . . . . . . . . . . . . . . . . . . . . . . 189

6.8 Conclusions. . . . . . . . . . . . . . . . . . . . . . . . . . . . . . . . . . 192

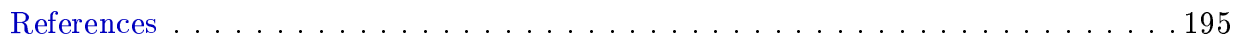


7 Paper 6. Energy-efficient control for an unmanned ground vehicle in a wireless sensor network

7.1 Introduction . . . . . . . . . . . . . . . . . . . . . 202

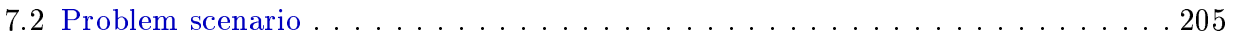

7.2.1 Time-varying network-induced delays, packet dropouts, and packet disorder . . 205

7.2 .2 Control structure . . . . . . . . . . . . . . . . . . . . . 207

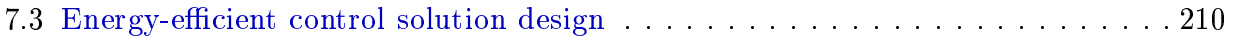

7.3 .1 Plant modeling. . . . . . . . . . . . . . . . . . . . . . . 210

7.3 .2 Event-triggered conditions . . . . . . . . . . . . . . . . . . . . 212

7.3.3 Time-varying dual-rate Kalman filter . . . . . . . . . . . . . . . . . . . 213

7.3.4 Pure Pursuit path tracking algorithm . . . . . . . . . . . . . . . . . 215

7.3.4.1 Differential kinematics. . . . . . . . . . . . . . . . . . . . . . 216

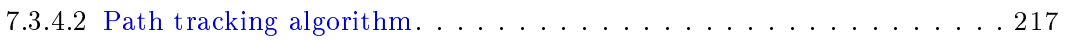

7.3 .5 Dual-rate controller . . . . . . . . . . . . . . . . . . . 220

7.4 Cost indexes for control performance and resource usage . . . . . . . . . . . 222

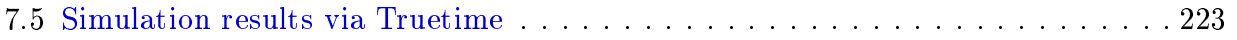

7.5.1 Application data . . . . . . . . . . . . . . . . . . 223

7.5.2 Truetime simulation and cost function evaluation. . . . . . . . . . 225

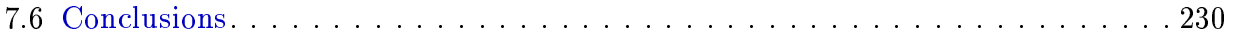

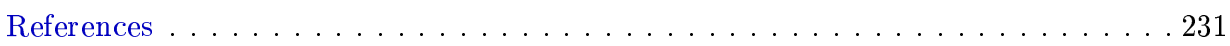

8 Paper 7. A remote control strategy for an autonomous vehicle with slow sensor using Kalman filtering and dual-rate control 237

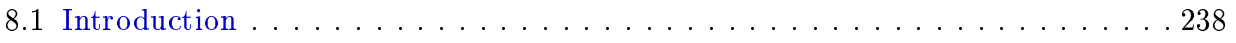

8.2 Problem Scenario. . . . . . . . . . . . . . . . . . . . . . . . 241

8.2 .1 Control Structure . . . . . . . . . . . . . . . . . . . . . . . . . 241

8.2.2 Time-Varying Delays, and Packet Disorder. . . . . . . . . . . . . 243

8.3 Motion Planning and Control Solution Design. . . . . . . . . . . . . . . 246

8.3 .1 Plant Modeling. . . . . . . . . . . . . . . . . . . . . . . . . . . . 246

8.3.1.1 Kinematic Model . . . . . . . . . . . . . . . . . . . . . . . . 246

8.3 .1 .2 Dynamic Model. . . . . . . . . . . . . . . . . . . . . . 247

8.3.2 Extended Kalman Filter, Including an $h$-Step-ahead State Prediction Stage $\ldots 248$

8.3.3 Pure Pursuit Path Tracking Algorithm . . . . . . . . . . . . . . . . . . . . . 251

8.3.4 Dual-Rate Controller . . . . . . . . . . . . . . . . . . . . . 253 
8.4 Cost Indexes for Control Performance . . . . . . . . . . . . . . . . . . . 254

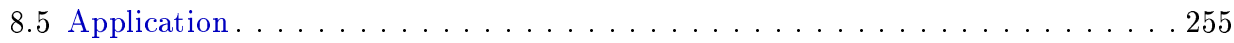

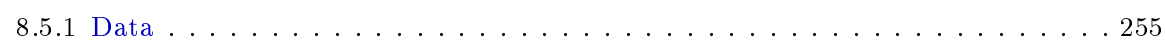

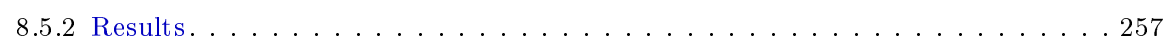

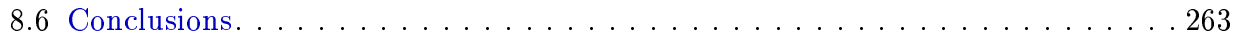

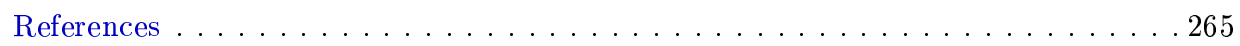

9 Conclusions 271

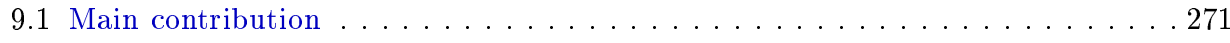

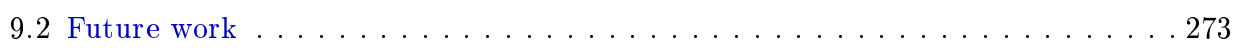

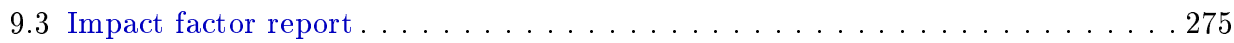

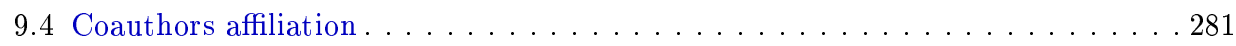




\section{List of Figures}

1.1 Conventional sampling . . . . . . . . . . . . . . . . . 1

1.2 Asynchronous sampling . . . . . . . . . . . . . 1

1.3 Multirate sampling . . . . . . . . . . . . . . . . . 2

1.4 Cyclic sampling . . . . . . . . . . . . . . . . 2

1.5 Random sampling. . . . . . . . . . . . . . . . . . . 3

1.6 Continuous control (left) and discrete control (right) configurations 3

1.7 Networked control system configuration . . . . . . . . . 4

1.8 Problems of NCS: Network access delay (A), Network transmission delay (B), Packet dropout (C), Packet disorder (D), Clock desynchronization (E) and Frequency difference between different clocks $(\mathrm{F}) \ldots \ldots \ldots \ldots \ldots \ldots$

1.9 Using network only to change reference and to monitor the system 7

1.10 NCS without local control . . . . . . . . . . . . 8

1.11 NCS with local open-loop control . . . . . . . . . . . 8

1.12 NCS with local closed-loop control . . . . . . . . . . . 9

1.13 Time-triggered control (left) and event-triggered control (right) configurations ................... 11

1.14 TTS transmissions . . . . . . . . . . . . . . . . 11

1.15 ETS transmissions . . . . . . . . . . . . . . 11

xxiii 
1.16 Periodic event-triggered control configuration . . . . . . . . 12

1.17 PETS transmissions (top) and overstepping the threshold without transmitting data in PETS (bottom) . . . . . . . . . . 13

1.18 Nominal case (no-delay, no-dropout) . . . . . . . . . . 14

1.19 Delay-dependent control . . . . . . . . . . . . . . . . . 15

1.20 Delay-independent control . . . . . . . . . . . . . . . 15

2.1 Quanser rotary inverted pendulum . . . . . . . . . . . 34

2.2 Top and front view . . . . . . . . . . . . . . . 35

2.3 Control structure in ideal conditions . . . . . . . . . . 36

2.4 Theta and alpha angles: $\mathrm{T}=10 \mathrm{~ms} \ldots \ldots \ldots \ldots$

2.5 Theta and alpha angles: $\mathrm{T}=15 \mathrm{~ms} \ldots \ldots \ldots \ldots$

2.6 Threshold-based communication flowchart . . . . . . . . . 40

2.7 Theta threshold . . . . . . . . . . . . . . . . . . 42

2.8 Alpha threshold . . . . . . . . . . . . . . . . . . . . 42

2.9 Control action threshold . . . . . . . . . . . . . . . . 43

2.10 Performance index . . . . . . . . . . . . . . . 43

2.11 Theta and alpha thresholds . . . . . . . . . . . 44

2.12 Theta and control thresholds . . . . . . . . . . . 45

2.13 Alpha and control thresholds . . . . . . . . . . . 45

2.14 Theta and alpha: Threshold control . . . . . . . . . . 46

2.15 Theta angle: Captured and transmitted . . . . . . . . . 47

2.16 Theta transmission flag $\ldots \ldots \ldots \ldots$. . . . . . . . 48

3.1 NCS scenario . . . . . . . . . . . . . . . . . 60

3.2 Prediction stage in detail . . . . . . . . . . . . . . 62 
3.3 No packet dropout $\left(d_{k}^{r l}=1\right) \ldots \ldots \ldots \ldots 63$

3.4 Packet dropout $\left(d_{k}^{r l}=0\right) \ldots \ldots \ldots \ldots \ldots$. . . . . . . 64

3.5 Cartesian robot (3D CRANE module) . . . . . . . . 75

3.6 Delay histogram . . . . . . . . . . . . . 76

3.7 Simulation results: nominal vs delay-dependent with packet dropouts and no prediction vs delay-dependent with packet dropouts with prediction . . . . . . . . . . . 77

3.8 Experimental results: nominal vs delay-dependent with packet dropouts and no prediction vs delay-dependent with packet dropouts with prediction . . . . . . . . . . . . 80

4.1 Generalized Dual-Rate System _. . . . . . . . . . . 90

4.2 Kranc's vector switch decomposition . . . . . . . . . . . 91

4.3 DRS Bode . . . . . . . . . . . . . . . . . 94

4.4 DRS Bode Interpretation. $w=3 R / s \ldots \ldots \ldots \ldots$

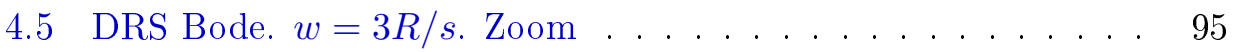

4.6 Output Components Sum Comparison . . . . . . . . . . 96

4.7 DRS Bode. Comparison of Methods . . . . . . . . . . 96

4.8 DRS Bode. Comparison of Methods . . . . . . . . . . . . . 97

4.9 Output Components Sum at $T_{0}$ Comparison . . . . . . . . 98

4.10 Simulation Diagram. Case $N_{y}=3 \ldots \ldots \ldots \ldots$

$4.11 T_{0}$ Bode Stability Margins Determination _ . . . . . . 100

4.12 Simulation of DRS with Stability Margins consideration . . . . 101

4.13 Metaperiod $T_{0}$ DRS Bode and the greatest Singular Value . . . 101

4.14 DR control scheme applied in example 1 . . . . . . . . . . 102

4.15 Time Response for different values of $N \ldots \ldots \ldots$ 
4.16 Root Locus for different values of $N \ldots \ldots$. . . . . . . . 104

4.17 Open Loop Bode diagrams for different values of $N$. . . . . . 104

4.18 Closed Loop Bode diagrams for different values of $N \ldots \ldots$

4.19 Closed Loop Dual-Rate Control. Non-conventional Dual-Rate Controller Structure used in example 2 . . . . . . . . . . 106

4.20 Time Response for different values of $N \ldots \ldots$. . . . . 107

4.21 Root Locus for different values of $N \ldots \ldots$. . . . . . 107

4.22 Open Loop Bode diagrams for different values of $N \ldots \ldots$. . 108

4.23 Closed Loop Bode diagrams for different values of $N \ldots \ldots$

$5.1 \quad$ NCS scenario . . . . . . . . . . . . . . . . . . . . 120

$5.2 \quad$ Packet dropout $\left(d_{k}^{r l}=0\right) \ldots \ldots \ldots \ldots \ldots$. . . . . . . 124

$5.3 \quad$ No packet dropout $\left(d_{k}^{r l}=1\right) \ldots \ldots \ldots \ldots$. . . . . . . 124

5.4 Cartesian robot (3D CRANE module) $\ldots \ldots \ldots \ldots$

5.5 Delay histogram . . . . . . . . . . . . . . . . 138

5.6 Comparison: nominal vs no prediction vs delay-dependent vs delay-independent . . . . . . . . . . . . . . . . . 139

5.7 Comparison: nominal vs delay-independent (model mismatch) . 142

5.8 Comparison: nominal vs no prediction vs delay-dependent vs delay-independent . . . . . . . . . . . . . . . 145

5.9 Comparison (Lissajous curves): nominal vs no prediction vs delay-dependent vs delay-independent . . . . . . . . . . . 146

6.1 Fast digital control . . . . . . . . . . . . . . . . . . . 159

6.2 Dual-rate inferential control . . . . . . . . . . . . . 160

6.3 Model-based dual-rate control . . . . . . . . . . . . . . . . 161

6.4 Generalized dual-rate system . . . . . . . . . . . . 162 
6.5 Kranc vector switch decomposition. General case . . . . . . . 163

$6.6 \mathrm{Y} / \mathrm{R}$ MBDR lifted process $\ldots \ldots \ldots \ldots \ldots$

$6.7 \mathrm{Y} / \mathrm{R}$ IC lifted process . . . . . . . . . . . . . . . . 173

$6.8 \mathrm{Y} / \mathrm{d}$ MBDR lifted process $\ldots \ldots \ldots \ldots \ldots \ldots$

$6.9 \mathrm{Y} / \mathrm{d}$ IC lifted process $\ldots \ldots \ldots \ldots \ldots$

6.10 Example 1. Time response without MPM . . . . . . . . . 182

6.11 Example 1. Time response with MPM . . . . . . . . . . . 182

6.12 Example 1. Y/R DR systems Bode. Methods comparison . . . 183

6.13 Example 1. MBDR case QFT analysis . . . . . . . . . . 183

6.14 Example 1. IC case QFT analysis . . . . . . . . . . . . . 184

6.15 Disturbance rejection comparison . . . . . . . . . . . . . 184

6.16 Example 2. Time response without MPM . . . . . . . . . 185

6.17 Example 2. Time response with MPM . . . . . . . . . 186

6.18 Example 2. Y/R DR systems Bode. Methods comparison . . . 186

6.19 Example 2. IC case QFT analysis . . . . . . . . . . . . . 187

6.20 Example 2. MBDR case QFT analysis . . . . . . . . . 187

6.21 Autonomous robot used . . . . . . . . . . . . . . . . . 188

$6.22 \mathrm{UGV}$ QFT analysis . . . . . . . . . . . . . . . . . 191

6.23 UGV Path tracking . . . . . . . . . . . . . . . . . . 191

6.24 UGV. Linear and Non-linear servomotors comparison . . . . . . 192

7.1 Energy-efficient control system for a WSN . . . . . . . . . 206

7.2 Structure of the Time-Varying Dual-Rate Kalman Filter . . . . 214

7.3 Circumference between current point and target point . . . . 217

7.4 Structure of the Pure Pursuit path tracking algorithm . . . . . 219 
7.5 Structure of the dual-rate controller . . . . . . . . . . . . . 221

7.6 Time-triggered single-rate control at period NT (no noise, no disturbance, no delays, no dropouts) . . . . . . . . . 225

7.7 Time-triggered single-rate control at period $T$ (no noise, no disturbance, no delays, no dropouts). Nominal performance . . . . 226

7.8 Time-triggered dual-rate control (no noise, no disturbance, no delays, no dropouts $\ldots \ldots$. . . . . . . . . . . 226

7.9 Time-triggered dual-rate control (no noise, no disturbance, delays, dropouts) . . . . . . . . . . . . . . 227

7.10 Time-triggered dual-rate control, with TVDRKF, and packetbased control (noise, disturbance, delays, dropouts) . . . . . . 227

7.11 Disturbance estimation by TVDRKF . . . . . . . . . . 228

7.12 Periodic event-triggered dual-rate control, with TVDRKF, and packet-based control (noise, disturbance, delays, dropouts) . . . 228

8.1 Remote control structure for an autonomous vehicle . . . . . 241

8.2 Time axis example for the remote-to-local channel . . . . . . . 244

8.3 Time axis example for the local-to-remote channel . . . . . . 245

8.4 Lego robot. . . . . . . . . . . . . . . . . 256

8.5 Results for the single-rate control at $T=0.1 \mathrm{~s} \ldots \ldots 258$

8.6 Results for the single-rate control at $N T=0.2 \mathrm{~s} \ldots \ldots 258$

8.7 Results for the dual-rate control at $N T=0.2 \mathrm{~s}$ and $T=0.1 \mathrm{~s} .259$

8.8 Results for the dual-rate control with delays . . . . . . . . . 259

8.9 Results for the control proposal (EKF, prediction stage, and packet-based control) with delays and a lower level of noise . . 260

8.10 Control signal for the control proposal (EKF, prediction stage, and packet-based control) with delays and a lower level of noise 260 
8.11 Results for the control proposal (EKF, prediction stage, and packet-based control) with delays and a higher level of noise . . 261

8.12 Control signal for the control proposal (EKF, prediction stage, and packet-based control) with delays and a higher level of noise 262

9.1 First page of paper 1 published in ISA Transactions . . . . . 275

9.2 First page of paper 2 published in ISA Transactions . . . . . 276

9.3 First page of paper 3 published in International Journal of General Systems . . . . . . . . . . . . . . . . . 276

9.4 First page of paper 4 published in Information Sciences . . . . . 277

9.5 First page of paper 5 published in ISA Transactions . . . . 277

9.6 First page of paper 6 published in Journal of Sensors . . . . . 278

9.7 First page of paper 7 published in Sensors . . . . . . . . . . 279 



\section{List of Tables}

2.1 Alpha and control action thresholds . . . . . . . . . . . 48

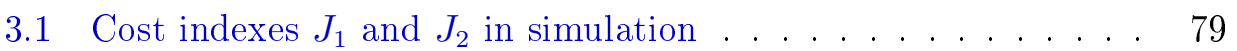

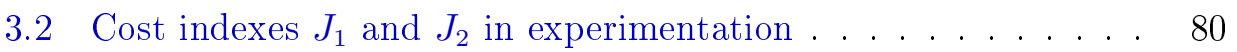

5.1 Simulation: accumulated error $E_{Y}$ and cost index $J_{1}$; overshot $O_{Y}$ and cost index $J_{2} \ldots \ldots \ldots \ldots \ldots 14 \ldots \ldots$

5.2 Accumulated error $E_{W}$ and cost index $J_{3} \ldots \ldots \ldots \ldots$

5.3 Overshot $O_{W}$ and cost index $J_{4} \ldots \ldots \ldots \ldots$

5.4 Experiment: accumulated error $E_{Y}$ and cost index $J_{1}$; overshot $O_{Y}$ and cost index $J_{2} \ldots \ldots \ldots \ldots \ldots \ldots$

$7.1 \quad$ Cost indexes . . . . . . . . . . . . . . . . . . 229

8.1 Example for $h=1 \ldots \ldots \ldots \ldots$. . . . . . . . . 261

8.2 Cost indexes for each scenario . . . . . . . . . . . . . 262

9.1 Quality metrics of the publications. . . . . . . . . . 280 



\section{List of Abbreviations}

$\begin{array}{ll}\text { A/D } & \text { Analog-to-digital } \\ \text { AV } & \text { Autonomous vehicle } \\ \text { CA } & \text { Controller-to-actuator link } \\ \text { CAD } & \text { Computer-aided design } \\ \text { D/A } & \text { Digital-to-analog } \\ \text { DR } & \text { Dual-rate } \\ \text { DRS } & \text { Dual-rate system } \\ \text { EKF } & \text { Extended Kalman filter } \\ \text { ESO } & \text { Extended state observer } \\ \text { ETC } & \text { Event triggered control } \\ \text { ETS } & \text { Event triggered sampling } \\ \text { FR } & \text { Frequency response } \\ \text { IAE } & \text { Integral of absolute error } \\ \text { IC } & \text { Inferential control } \\ \text { IMU } & \text { Inertial measurement unit } \\ \text { IP } & \text { Internet protocol } \\ \text { IRC } & \text { Instantaneous rotation center } \\ \text { LAD } & \text { Look ahead distance } \\ \text { LMI } & \text { Linear matrix inequalities } \\ \text { LQR } & \text { Linear-quadratic regulator } \\ \text { LTI } & \text { Linear time-invariant } \\ \text { LTV } & \text { Linear time-varying } \\ \text { MBDR } & \text { Model-based dual-rate } \\ \text { MIMO } & \text { Multiple-input multiple-output } \\ \text { MPM } & \text { Model plant mismatching } \\ \text { MR } & \text { Multi-rate } \\ \text { MRIC } & \text { Multi-rate input control } \\ & \end{array}$

xxxiii 
MRS Multi-rate system

NCS Networked control system

PETC Periodic event-triggered control

PETS Periodic event triggered sampling

PWM Pulse-width modulation

QFT Quantitative feedback theory

RIP Rotary inverted pendulum

SC Sensor-to-controller link

SISO Single-input single-output

SN Sensor network

SVD Singular value decomposition

TCP Transmission control protocol

TTC Time triggered control

TTS Time triggered sampling

TVDRKF Time-varying dual-rate Kalman filter

TVDT Time-varying discrete-time

UDP User datagram protocol

UGV Unmanned ground vehicle

VSD Vector switch decomposition

WSN Wireless sensor network

ZOH Zero-order hold 


\section{Chapter 1}

\section{Introduction}

\subsection{Sampling methods}

Conventional sampled systems are known as those that include devices whose variables are sampled every $\mathrm{T}$ (period) units of time (see Figure 1.1). This conventional sampling will be synchronous when all the system samplers are synchronized, and asynchronous when there is $\Delta$ delay in the sampling of the variables of different devices (see Figure 1.2).

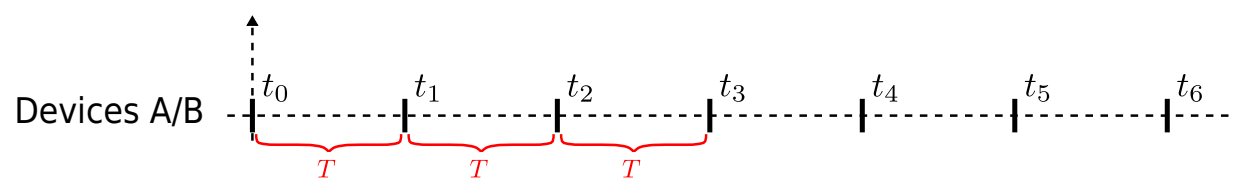

Figure 1.1: Conventional sampling

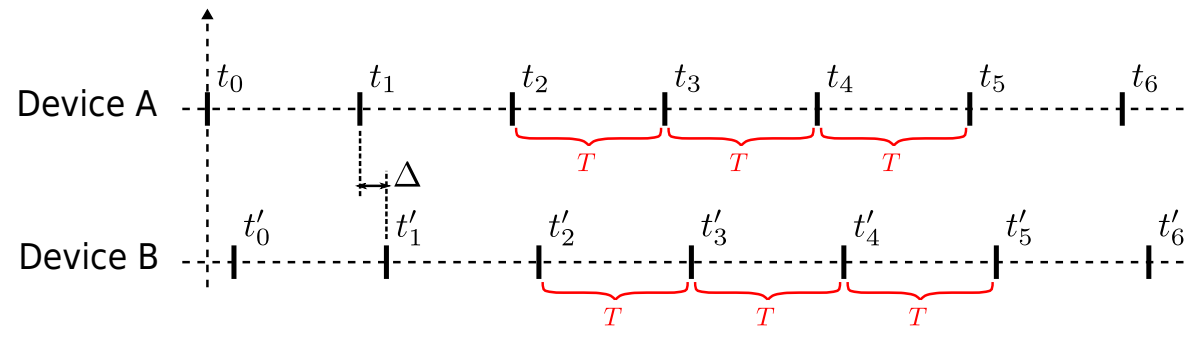

Figure 1.2: Asynchronous sampling

Multirate sampled systems are known as sampled systems that include devices whose variables are updated at different frequencies (see Figure 1.3). Cyclic 
sampling can be considered an extension of multirate systems. It is a type of sampling in which variables are sampled at variable time intervals with an NT global period, sometimes named metaperiod (see Figure 1.4).

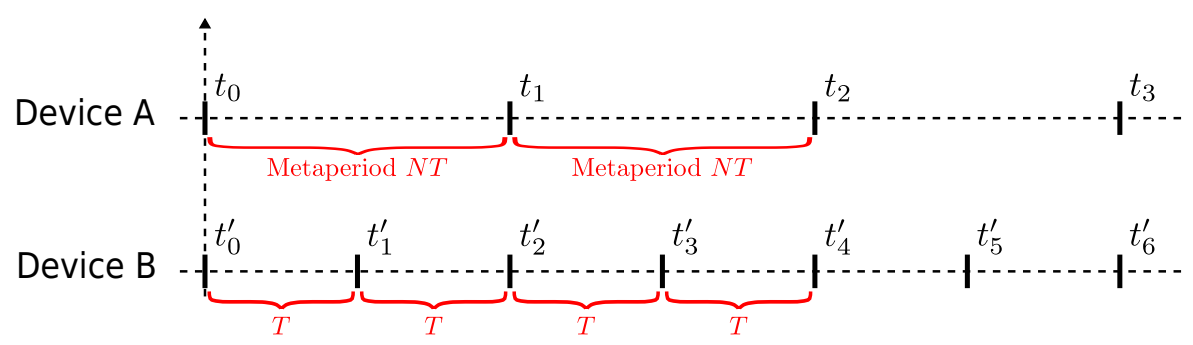

(a) Multirate sampling. NT local

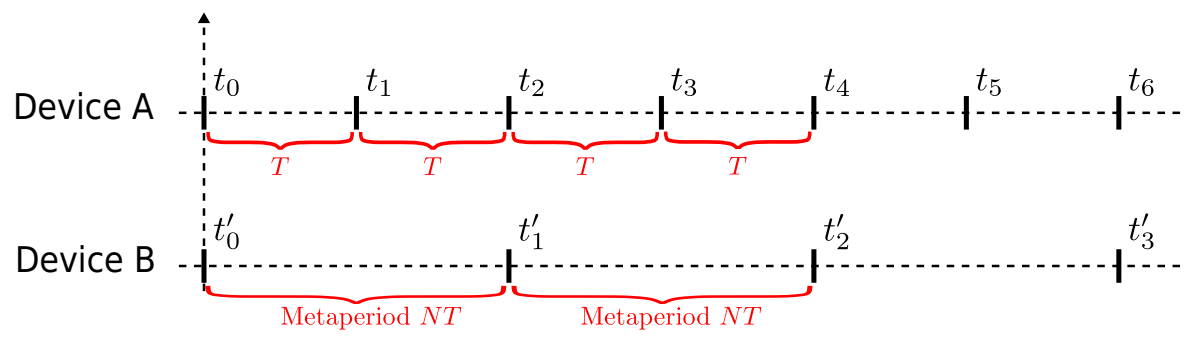

(b) Multirate sampling. NT remote

Figure 1.3: Multirate sampling

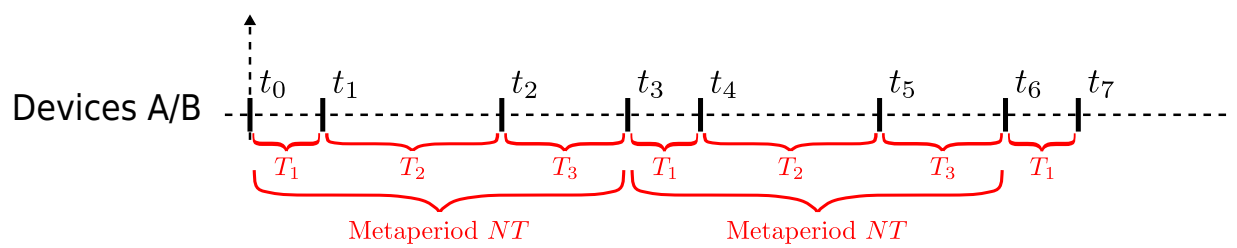

Figure 1.4: Cyclic sampling

Lastly, systems sampled randomly are those in which sampling is done at variable time intervals (without the restriction of having to form a sampling pattern that is repeated every global T period) (ver Figure 1.5). 


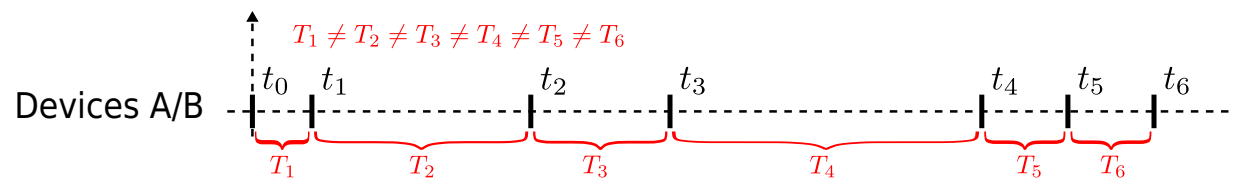

Figure 1.5: Random sampling

\subsection{Networked control systems}

This section introduces networked control systems compared to conventional control, and it lists the advantages and disadvantages of its use as well as its most common configurations.

In continuous control (Figure 1.6, left) all variables are continuous; this is a type of control that has fallen into disuse due to the emergence of digital computers as control elements in the 80's. Thus, discrete control is born (Figure 1.6, right), requiring $\mathrm{A} / \mathrm{D}$ and $\mathrm{D} / \mathrm{A}$ signal converters, that work at a sampling period.

Whether the control system is continuous or discrete, conventional control is characterized by having a direct connection between the controller and the process. Nevertheless, networked control introduces a shared communication medium between the control system's devices and the process. (Figure 1.7).
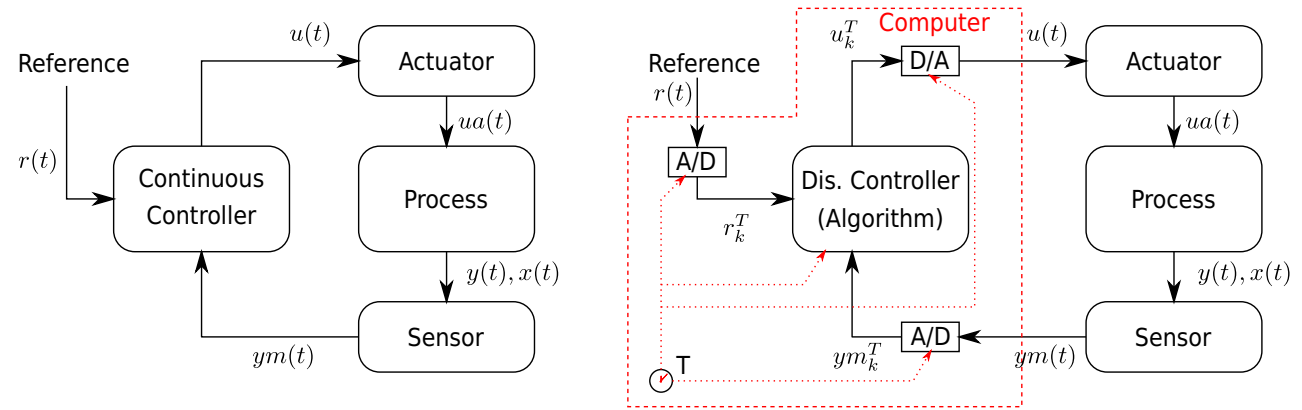

Figure 1.6: Continuous control (left) and discrete control (right) configurations 


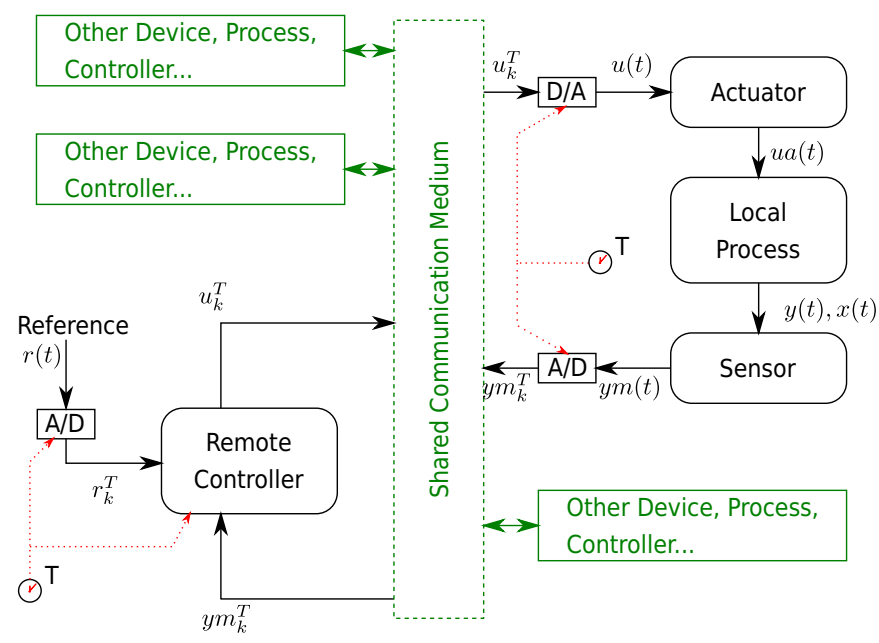

Figure 1.7: Networked control system configuration

\subsubsection{Advantages}

Networked control systems (NCS) is a relatively new technique. When compared with local conventional control it has the following obvious advantages in a number of fields such as robotics, avionics, chemistry, medical, and fields that use systems which require the optimization of costs, maintenance or space:

- Wiring reduction. By not using exclusive links for each of the variables communications in the system, this leads to a reduction in its wiring, which has the following direct advantages:

- The wiring cost is more inexpensive.

- The final system takes up less space and is lighter; this translates into an increase in the autonomy of the batteries it may have.

- The installation of the system is easier.

- Flexibility. The system is more robust to changes and much more scalable due to the modularization of the different systems. In some cases, the control centralization of an entire series of different remote elements is possible. 
- Wireless links enable to control systems located at large distances inaccessible through direct wiring, or whose control at short distances is dangerous.

\subsubsection{Disadvantages}

Despite the advantages that networked control systems have, they also present several disadvantages that must be assessed and will be explained in this section. There are two problems related to the amount of data to send to the communication system which are immediately detected:

- The first problem is the bandwidth limitation. Any communication system can only send a certain amount of information by unit of time. Furthermore, in the event of sharing the communication link with other devices, time usage must be divided between each one of these. This means that the amount of information sent in the control loop is reduced as the number of devices connected to the network is increased.

- The second problem, inherited by digital control and aggravated by the NCS, is the quantification. The information sent to the communication medium must be coded. This information will be transmitted trying to use the least amount of bytes in order to use the lowest bandwidth as possible. This truncates the magnitude of information, which will affect the behavior of the process.

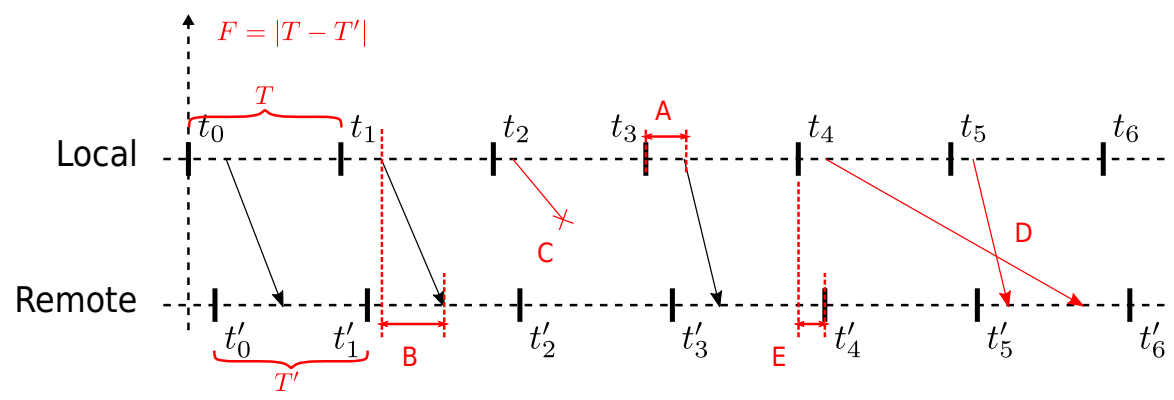

Figure 1.8: Problems of NCS: Network access delay (A), Network transmission delay (B), Packet dropout (C), Packet disorder (D), Clock desynchronization (E) and Frequency difference between different clocks $(\mathrm{F})$

Other problems regarding time limitations, depicted in Figure 1.8 are: 
- Access delay to the network (A) or transmission delay in the network (B).

- Access delay is the time elapsed between the signal is tried to be sent to the communication system and the moment it is begun to be sent. This delay is set by the communication system and might be explained by another device using the network when the data packet is attempted to be sent. Access delay is usually of a random nature, which makes its modeling difficult.

- Transmission delay is the time elapsed between the moment the transmission of a data packet begins and the moment it reaches its destination. This delay is set by the communication system's bandwidth. This bandwidth is characterized by a known time unit per byte.

As the sum of these two delays increases, it will have a negative influence on the system's behavior, and therefore it will have to be taken into account in the controller design.

- Packet dropout $(\mathrm{C})$. Certain systems such as UDP transmissions, where there is no packet confirmation, or in communications that might involve buffer overloads can cause packet dropouts. In a similar way to delays, this phenomenon will worsen the control system's behavior. In some communication systems the dropouts may be statistically determined, and must be taken into account in the controller algorithm design.

- Packet disorder (D). In some communication systems without flow control a data packet can reach its destination before another which should have arrived sooner. Usually an old packet received after a newer one is discarded, considering it a packet dropout. It can never be considered as the most current packet. There are existing methods to determine the order of the packets such as adding a time-stamp to the packet data before it is sent.

- Clock desynchronization (E) and frequency difference between different clocks $(\mathrm{F})$. As an exclusive link between the different parts of the control loop doesn't exist, the clocks that manage these parts are not synchronized. Also, their tick times may not be identical, which makes their desynchronization variable in time. Generally, if there is a way to synchronize the clocks, it must be done. If not, this phenomenon must be taken into account when designing the controller. 


\subsubsection{Types of networked control systems}

As mentioned before, NCS are characterized by the presence of a shared communication link between the control and plant. This implies the partial or total presence of a remote controller, which is physically separated from the process to be controlled and communicated via a non-exclusive link.

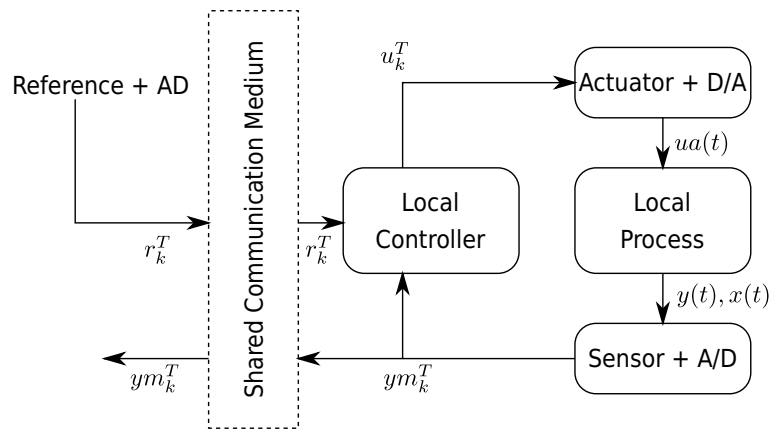

Figure 1.9: Using network only to change reference and to monitor the system

Ocasionally, the shared link is only used for monitoring tasks and reference changes (Figure 1.9). In these cases, the control structure is not really an NCS. To be considered one, it should separate, at least, part of the control loop. Thus, there must be a remote part of the control system and, optionally, a local control structure to the plant can exist. This local control is connected to the plant through an exclusive link, so it lacks the inconveniences described in subsection 1.2.2. Depending on the existence or not and the type of this local control, there will be different types of NCS.

- NCS without local control. This structure is given when the controller is totally located in the remote part of the NCS and communicates with the controlled process through the shared network (Figure 1.10).

If the shared communication medium has good behavior (it doesn't add considerable delays in communication, it doesn't lose packets...) the remote controller performs in a similar way to a conventional discretecontrol loop. Nevertheless, as network-induced problems increase, the system's behavior worsens, as the samples captured in the plant are totally sent without being used in any other operation in the local part of the control loop. Also, the control actions received are directly applied to the plant without being processed in any way. 


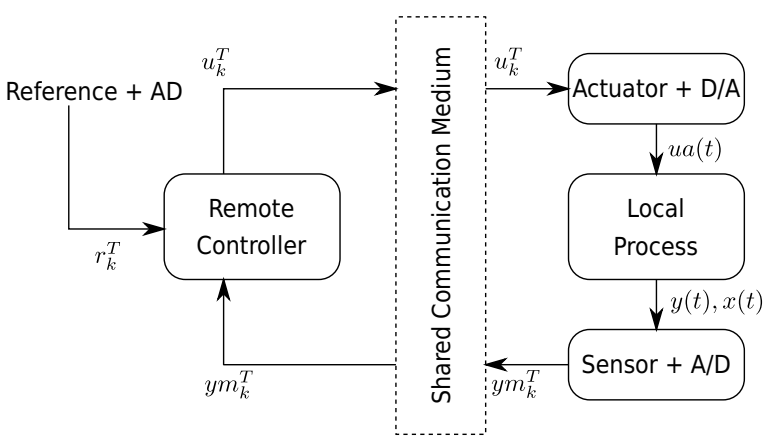

Figure 1.10: NCS without local control

This type of control structure is used when the local part of the loop only disposes of sensors and actuators without computation ability or with a reduced ability to do so. NCS without local control is used in paper 1 .

- NCS with local open-loop control (Figure 1.11). In this case, the local part of the control system is provided with a computation element. Once it receives the control actions from the remote controller, this local part is in charge of generating the actions that will really be applied to the plant, which are generally different.

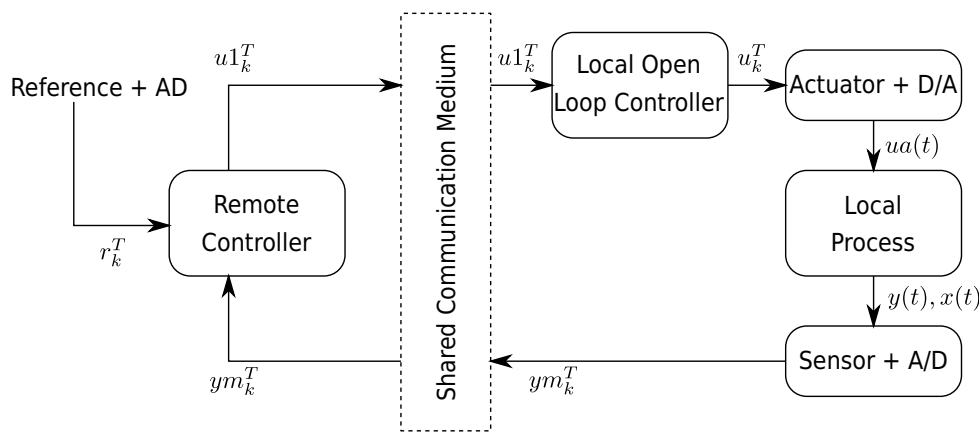

Figure 1.11: NCS with local open-loop control

This improvement of NCS without local control has the advantage, aside from receiving remote control actions, of knowing, for example, the roundtrip delay, or if the data was lost in that period. Consequently, a correction of the control actions can be made. This type of control system has the same disadvantage as the previous one; the sensor variables are not lo- 
cally used and their effects on the control are unknown until they are returned by the remote controller.

At this time the following obvious question arises: As the local part contains an element with computational ability, why is the controller algorithm moved to the remote part and it is not implemented entirely in the local part? This control structure makes sense when the local part's computational abilities are limited and the control strategy is complex. As an example, in the trajectory control of an UGV on a map, the microcontroller that commands the vehicle may be inexpensive and only allow certain basic calculations. It may not be able to make complex trajectory decision ones that could depend on factors such as the climate or GPS position which could require corrections. In this case the remote part would perform the bulk of the computational operations and the local part would only make corrections knowing if the data was lost, delayed or disordered. This type of control structure has been used in papers 2, 4 and 6 .

- NCS with local closed-loop control (Figure 1.12). Despite the previous structure being provided by a local element with computational ability, the data it receives comes from the shared communication medium, and this has its own limitations. NCS with local closed-loop control considers the existence of a feedback loop without involving the shared communication medium in order to improve the system's performances. In this way, the local control loop operates as an ideal loop (with no delays, dropouts, disorder...) and all its elements are synchronized.

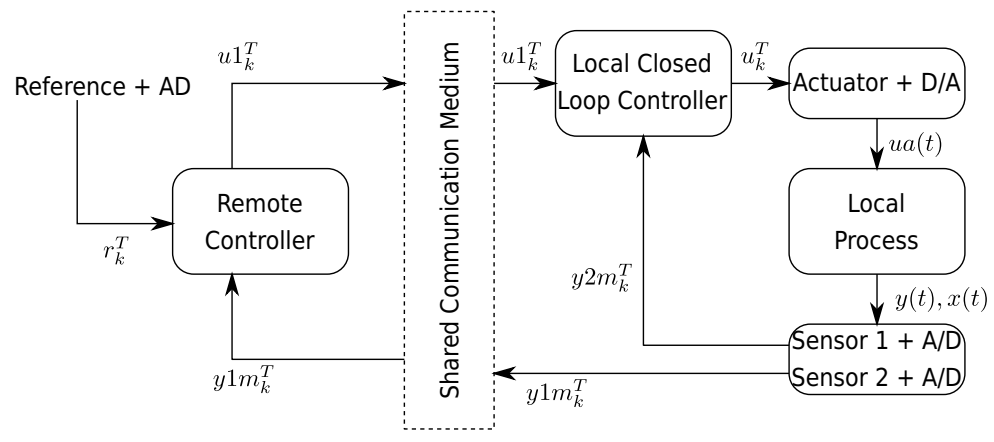

Figure 1.12: NCS with local closed-loop control

The justification of using this type of control structure against the use of NCS without local control is the same as in NCS with local open-loop 
control. However, NCS with local closed-loop control is a more versatile strategy despite its cost being generally increased. As an example, NCS with local closed-loop control could be used to locally control unstable or high-frequency elements of a system. The control of these elements remotely would not be possible due to problems of the shared communication medium. The parts of the control system requiring a high computation ability and control slow or stable elements of the system could be made remotely. Paper 7 uses this control strategy.

\section{$1.3 \quad$ Event-triggered control}

This subsection presents event-triggered control compared to time-triggered control. It also emphasizes periodic event-triggered control (PETC) due to its higher practical usage.

\subsubsection{Time Triggered Control (TTC) and Event Triggered Control (ETC)}

The recent proposal of using event-triggered sampling (ETS), instead of timetriggered sampling (TTS) in control systems has become a trending research area. While in the TTS strategy, the plant is periodically sampled (figures 1.13, left and 1.14), in the ETS approach, the plant is only sampled "when necessary", that is, when state or output variables satisfy a certain event condition (figures 1.13, right and 1.15; note that, in some figures of this subsection, notation TRH means threshold). In this way, ETS (compared to TTS) is better equipped to lead to a reduction of resource utilization. However, the ETS strategy manages less system information, and therefore, control performance may be worsened (compared to the desired one, which is defined by the TTS case) if the ETS schemes are not designed appropriately. In particular, model based control techniques for the ETS strategy might be beneficial to guarantee a satisfactory control performance.

Figure 1.13, right shows the event-based condition at the sensor device. Additionally, another condition may be defined for the controller output. 

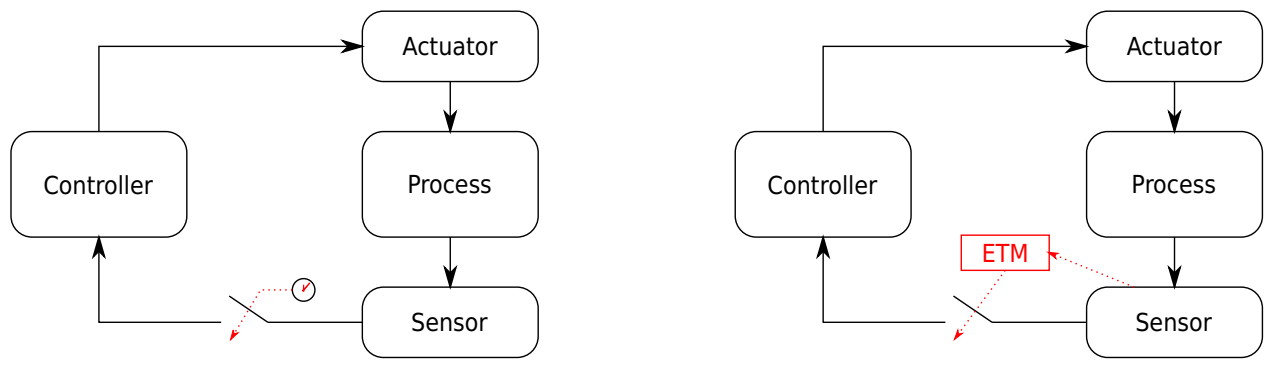

Figure 1.13: Time-triggered control (left) and event-triggered control (right) configurations

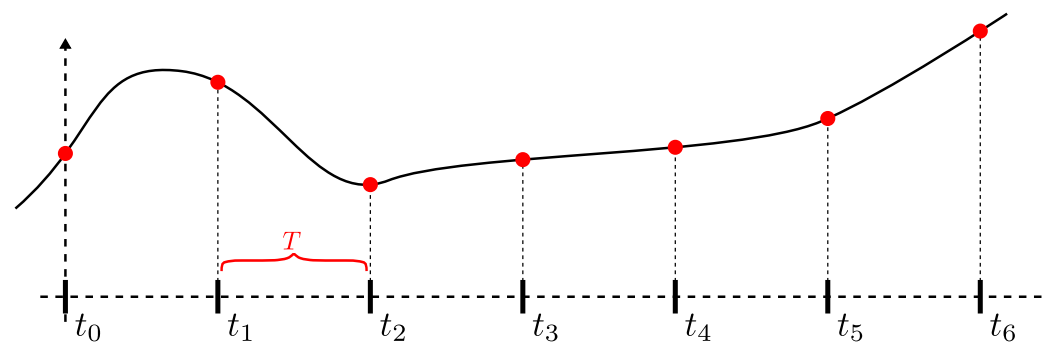

Figure 1.14: TTS transmissions

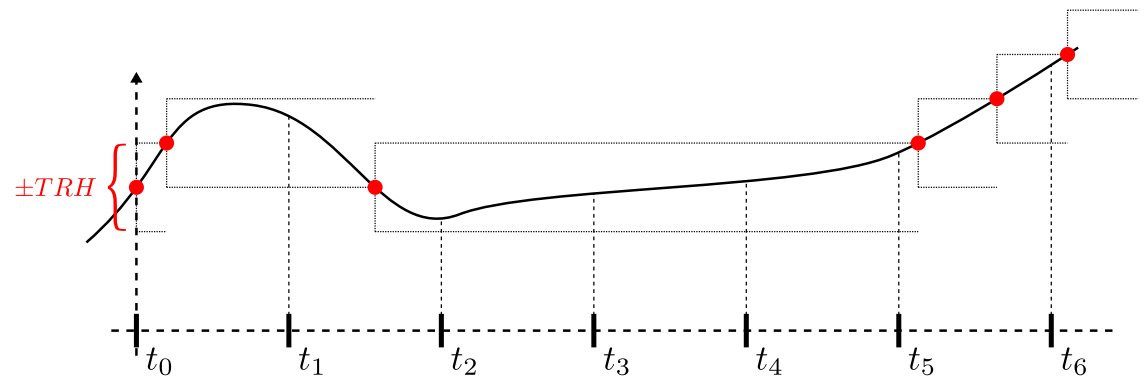

Figure 1.15: ETS transmissions

\subsubsection{Periodic Event-Triggered Control}

Integrating ideas from TTS and ETS paradigms results in periodic eventtriggered sampling (PETS), where the event-triggering conditions are evaluated periodically (figures 1.16 and 1.17). In most of ETC schemes the eventtriggering condition has to be verified continuously, which implies an overuse of devices (the opposite effect to the intended one). This is one of the main 
reasons of using PETS. In fact, Paper 1 formally describes PETS (In the paper it is named threshold-based control) and in papers 2,4 and 6 it is used considering an unsent packet as a packet dropout from the sensor or the controller.

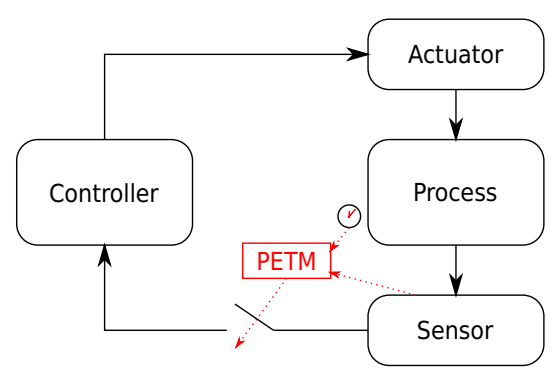

Figure 1.16: Periodic event-triggered control configuration

Nevertheless, in periodic event-triggered control (PETC) it is essential to take a specific problem into account: if the sampling time is sufficiently long, a certain signal could overcome the defined threshold and return beneath it between a sampling period and the next. This could occur, for example, due to a perturbance $(1.17$, bottom). In this case the event would not be triggered and the destination device would not receive the information. This must be considered when selecting the sampling time. 

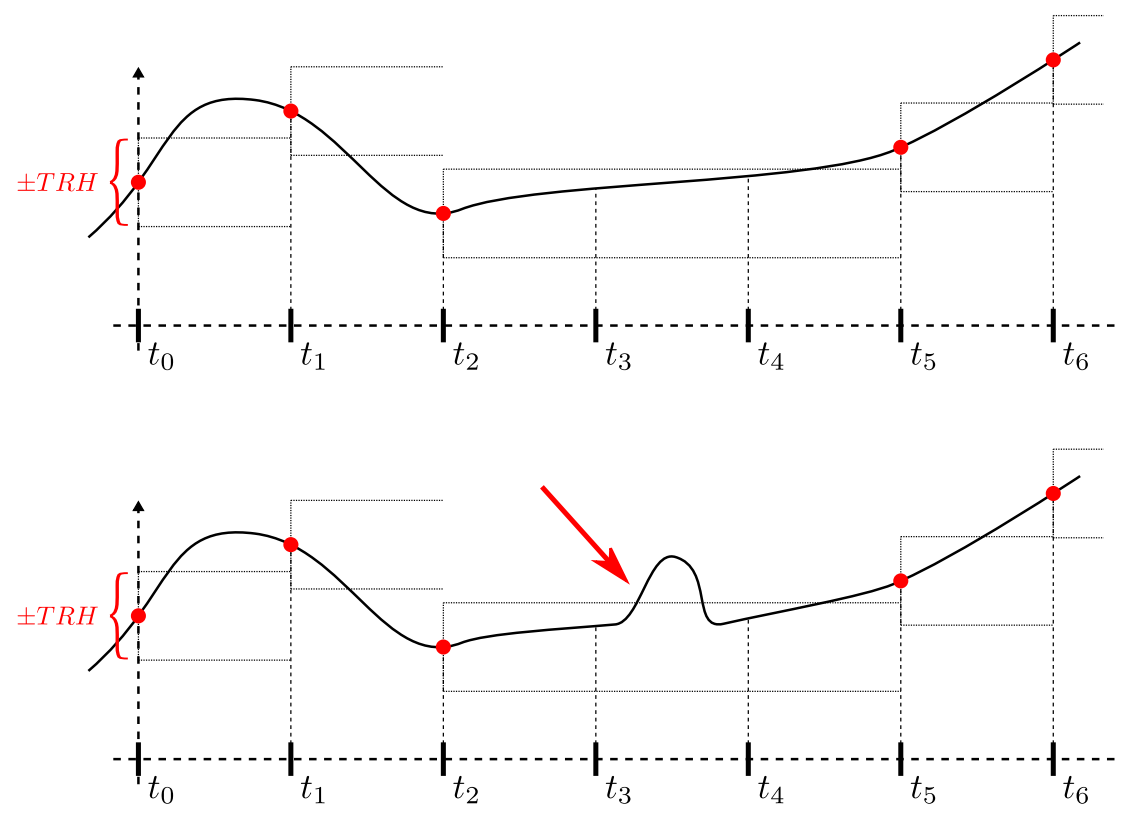

Figure 1.17: PETS transmissions (top) and overstepping the threshold without transmitting data in PETS (bottom) 


\subsection{Packet-based control}

Packet-based control is a technique which enables the decrease of the communication rate by simultaneously sending a set of data in each transmission. This strategy can be useful not only for resource-saving purposes but also for complementing prediction-based control techniques, since the controller is allowed to send a set of control signal estimations in a packet through the network to the actuator.

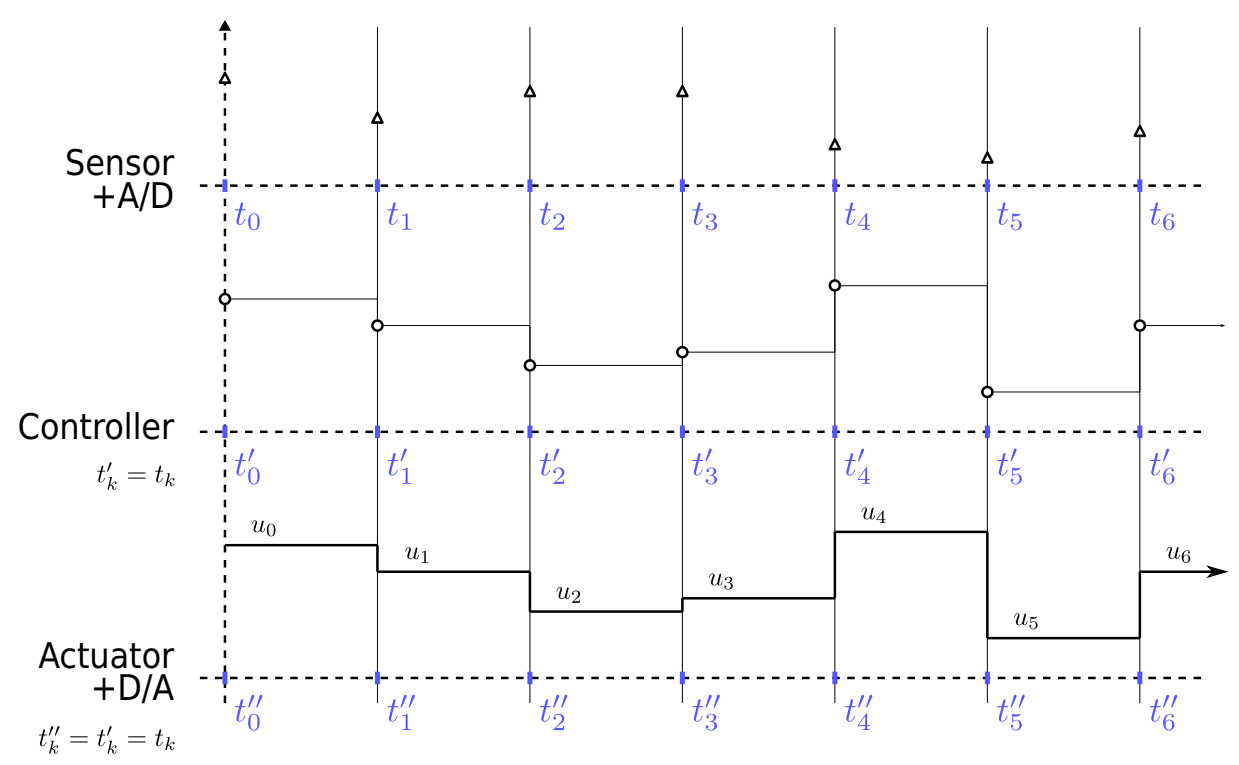

Figure 1.18: Nominal case (no-delay, no-dropout)

Starting from the nominal case (no-delay, no-dropout) in Figure 1.18 two different cases are proposed to be considered when time-varying delays and dropouts appear:

a) Delay-dependent approach (Figure 1.19): The control signal is returned to compensate for the delay (for example, adopting a gain scheduling approach such as in paper 2). Later, the signal is applied to the process by the actuator in a non-uniform way, that is, the last control action of the previous sensor period is held while no new one can be applied due to the delay. When a dropout is detected (for example, after expiring a supposed maximum waiting time $\tau_{\max }$ ), predictor-based control techniques can be integrated to generate a control signal estimate to be applied. 


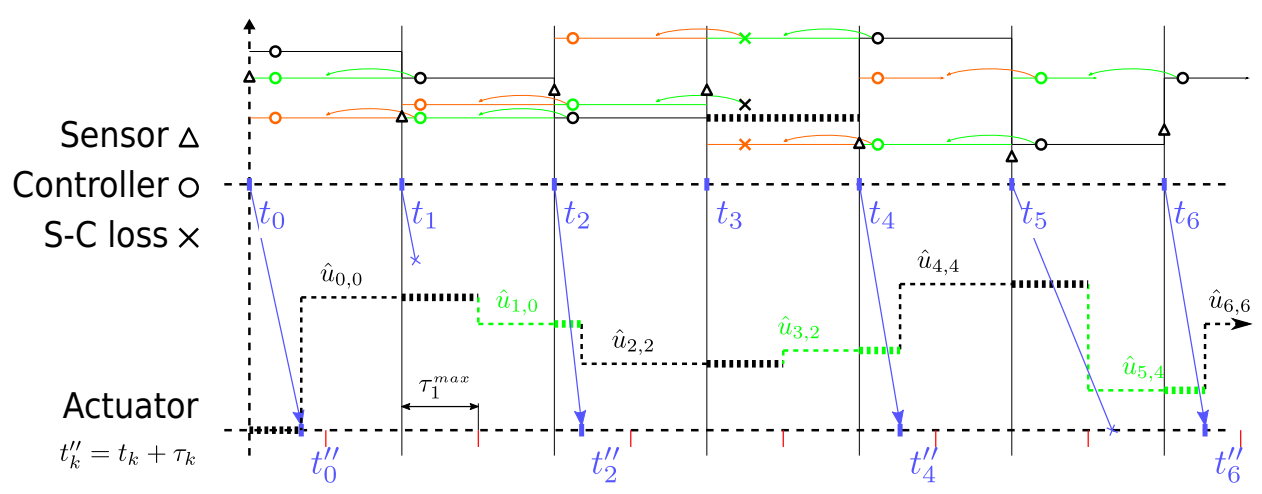

Figure 1.19: Delay-dependent control

b) Delay-independent approach (Figure 1.20): if dropout occurs, the actuator injects a control action estimate following a uniform pattern (i.e., from the beginning of the actuator period). The control action was received in a previous successful delivery. If no dropout occurs, the actuator injects the control action following a non-uniform pattern, that is, at the beginning of the period an estimated control action is injected, and once the new packet is received after the delay, the estimated control action is replaced with the the most recent one. Assuming neither model uncertainties nor disturbances, both signals should be very similar. This approach was used in paper 4 , and in paper 6 by means of a time-varying dual-rate Kalman filter. In paper 7, although no dropouts were considered, the approach was also used via an Extended Kalman filter to face time-varying delays.

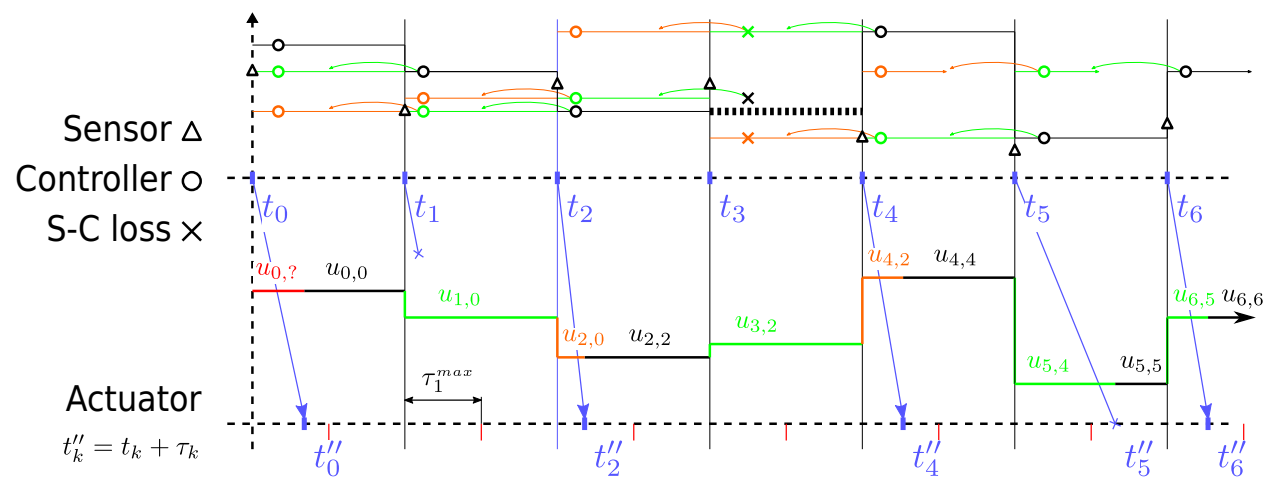

Figure 1.20: Delay-independent control 


\subsection{Aspects on frequency response}

The main aspects on frequency domain treated in the doctoral work are presented in these papers:

1. Julián Salt and José Alcaina. "Dual-rate sampled-data systems. Some interesting consequences from its frequency response analysis". In: International Journal of General Systems 48.5 (2019), pp. 554-574. DOI: https://doi.org/10.1080/03081079.2019.1608984

2. Julián Salt et al. "Multirate control strategies for avoiding sample losses. Application to UGV path tracking". In: ISA Transactions (2020). ISSN: 0019-0578. DOI: https://doi.org/10.1016/j.isatra.2020.01.025

In the first paper, a new frequency response based procedure for SISO dual-rate systems (DRS) is presented. From this tool, and keeping classical frequency domain methods, interesting analysis conclusions about stability margins and performance prediction can be reached. Typical questions about DRS such as how the delay affects the control system, or what is the optimum relation between sampling periods, can be solved using this tool.

In the second paper, a novel quantitative feedback theory (QFT) method for dual-rate systems is introduced. The method uses a new dual-rate frequency response procedure, which is based on discrete lifting modeling, in order to compare dual-rate strategies for slowly measured systems. These systems come from a single-rate control when vacant or missing samples are produced due to any failure or deliberate loss. Concretely, the dual-rate strategies under study are dual-rate inferential control (IC) and model-based dual-rate control (MBDR). A discussion on robust stability and performance under sample losses, disturbances, and process uncertainty is reported, which leads to the main conclusion: MBDR outperforms IC. Additionally, experimental results for UGV path tracking reveals that dual-rate control is able to reach energy saving.

\subsection{Literature review}

\subsubsection{Papers on event-triggered control}

Event-triggered sampling (ETS) in control systems [3-5] has become a trending research area. ETS enables to reduce resource utilization, but less sys- 
tem information is managed, and therefore, control performance may be worsened (compared to the desired one, which is defined by the time-triggered case). In particular, model-based control techniques $[6,7]$ for the ETS strategy might be beneficial to guarantee a satisfactory control performance. In periodic event-triggered sampling (PETS), the event-triggering conditions are evaluated periodically. Different works on PETS can be found for linear systems (using, e.g., both state-feedback and output-feedback control solutions [5, 8, 9], observer-based control [6], multirate control [10], $H_{\infty}$ control [11], and Youla-Kucera-like parameterization techniques [12]) and for nonlinear systems (using, e.g., state-feedback control [13, 14], output-feedback control [15], and observer-based control [16]). PETS can be studied either in a continuous-time framework (see, e.g., [17]) or in a discrete-time one (see, e.g., [18]). Demonstrators of PETS schemes are rare; exceptions are [19-23].

\subsubsection{Papers on packet-based control}

Packet-based control [24-27] is a control strategy which enables to reduce communication rate preserving control performance. It is possible by generating a sequence of signals to be sent over the network simultaneously. One interesting feature of using this strategy together with prediction-based control techniques is that the control law can become delay-independent (i.e., the network-induced delay is not required to be measured and compensated for). This is very relevant for implementation purposes, since it makes the solution applicable to a wide range of networked control applications where the time delay is difficult to measure [28-30].

\subsubsection{Papers on multi-rate frequency analysis}

Slowly sampled systems are systems where some samples are lost with respect to the ideal measurement frequency due to different situations: outliers, delays, measurement noises, etc. These systems can be studied from different points of views: soft sensors [31], inferential control for multi-rate systems [32, 33], model-based dual-rate control [34], etc.

In this doctoral work, the inferential control strategy in [33] is compared to the model-based dual-rate control in [34] using frequency response FR analysis techniques. The inferential control works in such a way that the lost samples are provided by a process model. Only one of each $\mathrm{N}$ closed loop output samples can be measured. This is a dual-rate control problem, which can be solved by designing a convenient dual-rate controller as in [34]. 
The comparison between both control strategies faces robust stability and performance issues. QFT [35, 36] is used for this purpose, since it is an interesting tool to design robust controllers for processes with parametric uncertainty.

\subsubsection{Papers on prediciton-based techniques}

Prediction-based techniques are required when event-based control is used, since they are able to provide the system with relevant information on intersampling times. With such inter-sampling information, both resource saving and performance criteria can be guaranteed. There is some existing literature that addresses various challenges on the event-based state estimation problem. In [6], a Luenberger observer at the sensor device and a model-based predictor, that runs both at the sensor and the controller, were proposed to estimate the state of the plant. In [37], a nominal model of the system, which was incorporated into the actuator/controller node in order to estimate the state between non-periodic updating intervals with time-varying delays, is presented. In [38], an $H_{\infty}$ filter for NCS with communication delay is developed. In [39, 40], distributed event-triggered filters over sensor networks (SN) by employing $H_{\infty}$ control techniques are designed. In [41], distributed event-triggered filtering over wireless $\mathrm{SN}$ is proposed using a recursive algorithm to obtain an upper bound of the filtering error variance. In [42], event-triggered distributed set-membership estimators are constructed by taking the influence of unknown-but-bounded process and measurement noise into account. In [43, 44], networked estimation problems are presented using modified Kalman filters to deal with packet dropouts. In [45], a Gaussian sum filter is introduced. In [46], a modified Kalman filter with intermittent measurement updates is investigated for a household network. In [47], time-varying Kalman filters are used to estimate the state conditioned on the received measurements in a multi-agent framework. In [30], a recent line of research [46, 47] is considered, in which the celebrated Kalman filter is considered in a time-varying fashion.

\subsubsection{Papers on multi-rate control}

In dual-rate control $[34,48,49]$, a slower sensing rate in comparison to a faster actuation can be assumed. Dual-rate controllers in linear time-invariant setups have been considered, for instance, in an internal-model inferential structure in [33], in an algebraic transfer function setting in [50], or in a state-space representation in [51]. The event-triggered conditions at the sensor node can be periodically evaluated at the slow rate. Despite sensing in this way, the fast actuation enables achieving acceptable control properties [33, 52]. In addition, 
dual-rate control techniques provide twofold benefits: (i) to lessen the amount of transmissions through the network, which results in energy and bandwidth saving, and (ii) to elude packet disorder, selecting the sampling period to be larger than the largest round-trip time delay. Due to the broad knowledge of PID controllers in academic and industrial environments, dual-rate PID control schemes are considered in [1, 2, 28-30, 53]. 



\section{References}

[1] Julián Salt and José Alcaina. "Dual-rate sampled-data systems. Some interesting consequences from its frequency response analysis". In: International Journal of General Systems 48.5 (2019), pp. 554-574. DOI: https://doi .org/10.1080/03081079.2019.1608984 (cit. on pp. 16, 19).

[2] Julián Salt et al. "Multirate control strategies for avoiding sample losses. Application to UGV path tracking". In: ISA Transactions (2020). ISSN: 0019-0578. DOI: https : //doi .org/10 .1016/j . isatra. 2020.01.025 (cit. on pp. 16, 19).

[3] P. Tabuada. "Event-Triggered Real-Time Scheduling of Stabilizing Control Tasks". In: IEEE Transactions on Automatic Control 52.9 (Sept. 2007), pp. 1680-1685. ISSN: 2334-3303. DOI: 10.1109/TAC.2007.904277 (cit. on p. 16).

[4] Karl J. Aström. "Event Based Control". In: Analysis and Design of Nonlinear Control Systems: In Honor of Alberto Isidori. Ed. by Alessandro Astolfi and Lorenzo Marconi. Berlin, Heidelberg: Springer Berlin Heidelberg, 2008, pp. 127-147. ISBN: 978-3-540-74358-3. DOI: 10.1007/978-3540-74358-3_9 (cit. on p. 16).

[5] W. P. M. H. Heemels, J. H. Sandee, and P. P. J. Van Den Bosch. "Analysis of event-driven controllers for linear systems". In: International Journal of Control 81.4 (2008), pp. 571-590. DOI: 10.1080/00207170701506919. eprint: https://doi.org/10.1080/00207170701506919 (cit. on pp. 16, 17).

[6] W. P. M. H. Heemels and M. C. F. Donkers. "Model-based periodic eventtriggered control for linear systems". In: Automatica 49.3 (2013), pp. 698 711. ISSN: 0005-1098. DOI: https://doi.org/10.1016/j .automatica. 2012.11.025 (cit. on pp. 17, 18). 
[7] Jan Lunze and Daniel Lehmann. "A state-feedback approach to eventbased control". In: Automatica 46.1 (2010), pp. 211-215. ISSN: 0005-1098. DOI: https://doi.org/10.1016/j.automatica.2009.10.035 (cit. on p. 17).

[8] Xia Chen and Fei Hao. "Periodic event-triggered state-feedback and outputfeedback control for linear systems". In: International Journal of Control, Automation and Systems 13.4 (2015), pp. 779-787. ISSN: 2005-4092. DOI: 10.1007/s12555-013-0318-z (cit. on p. 17).

[9] W. P. M. H. Heemels, G. E. Dullerud, and A. R. Teel. " $\mathcal{L}_{2}$-Gain Analysis for a Class of Hybrid Systems With Applications to Reset and EventTriggered Control: A Lifting Approach". In: IEEE Transactions on Automatic Control 61.10 (Oct. 2016), pp. 2766-2781. ISSN: 2334-3303. DOI: 10.1109/TAC.2015.2502422 (cit. on p. 17).

[10] Ernesto Aranda-Escolástico et al. "Asynchronous periodic event-triggered control with dynamical controllers". In: Journal of the Franklin Institute 355.8 (2018), pp. 3455-3469. ISSN: 0016-0032. DOI: https://doi.org/ 10.1016/j.jfranklin.2018.01.037 (cit. on p. 17).

[11] A. Selivanov and E. Fridman. "Event-Triggered $H_{\infty}$ Control: A Switching Approach". In: IEEE Transactions on Automatic Control 61.10 (Oct. 2016), pp. 3221-3226. ISSN: 2334-3303. DOI: 10.1109/TAC.2015.2508286 (cit. on p. 17).

[12] Maor Braksmayer and Leonid Mirkin. "Redesign of Stabilizing DiscreteTime Controllers to Accommodate Intermittent Sampling **Supported by the Grand Technion Energy Program, grant 2020938." In: IFACPapersOnLine 50.1 (2017). 20th IFAC World Congress, pp. 2633-2638. ISSN: 2405-8963. DOI: https://doi.org/10.1016/j. ifacol.2017.08. 140 (cit. on p. 17).

[13] R. Postoyan et al. "Periodic event-triggered control for nonlinear systems". In: 52nd IEEE Conference on Decision and Control. Dec. 2013, pp. 7397-7402. DOI: 10.1109/CDC.2013.6761063 (cit. on p. 17).

[14] W. Wang et al. "Stabilization of nonlinear systems using state-feedback periodic event-triggered controllers". In: 2016 IEEE 55th Conference on Decision and Control (CDC). Dec. 2016, pp. 6808-6813. DOI: 10.1109/ CDC.2016.7799317 (cit. on p. 17).

[15] E. Aranda-Escolástico et al. "Design of periodic event-triggered control for polynomial systems: A delay system approach". In: IFAC-PapersOnLine 50.1 (2017). 20th IFAC World Congress, pp. 7887-7892. ISSN: 2405-8963. DOI: https://doi.org/10.1016/j.ifacol.2017.08.748 (cit. on p. 17). 
[16] Lucien Etienne, Stefano Di Gennaro, and Jean-Pierre Barbot. "Periodic event-triggered observation and control for nonlinear Lipschitz systems using impulsive observers". In: International Journal of Robust and Nonlinear Control 27.18 (2017), pp. 4363-4380. DOI: $10.1002 / \mathrm{rnc} .3802$. eprint: https ://onlinelibrary . wiley . com/doi/pdf/10.1002/rnc. 3802 (cit. on p. 17).

[17] W. P. M. H. Heemels, M. C. F. Donkers, and A. R. Teel. "Periodic EventTriggered Control for Linear Systems". In: IEEE Transactions on Automatic Control 58.4 (Apr. 2013), pp. 847-861. ISSN: 0018-9286. DOI: 10.1109/TAC. 2012.2220443 (cit. on p. 17).

[18] A. Eqtami, D. V. Dimarogonas, and K. J. Kyriakopoulos. "Event-triggered control for discrete-time systems". In: Proceedings of the 2010 American Control Conference. June 2010, pp. 4719-4724. DOI: 10.1109/ACC. 2010. 5531089 (cit. on p. 17).

[19] V. S. Dolk, J. Ploeg, and W. P. M. H. Heemels. "Event-Triggered Control for String-Stable Vehicle Platooning". In: IEEE Transactions on Intelligent Transportation Systems 18.12 (Dec. 2017), pp. 3486-3500. ISSN: 1558-0016. DOI: 10.1109/TITS.2017.2738446 (cit. on p. 17).

[20] B. A. Khashooei et al. "Suboptimal event-triggered control over unreliable communication links with experimental validation". In: 2017 3rd International Conference on Event-Based Control, Communication and Signal Processing (EBCCSP). May 2017, pp. 1-6. DOI: 10.1109/EBCCSP . 2017.8022833 (cit. on p. 17).

[21] Georg Alexander Kiener, Daniel Lehmann, and Karl Henrik Johansson. "Actuator saturation and anti-windup compensation in event-triggered control". In: Discrete Event Dynamic Systems 24.2 (2014), pp. 173-197. ISSN: 1573-7594. DOI: $10.1007 /$ s10626-012-0151-1 (cit. on p. 17).

[22] J. Araújo et al. "System Architectures, Protocols and Algorithms for Aperiodic Wireless Control Systems". In: IEEE Transactions on Industrial Informatics 10.1 (Feb. 2014), pp. 175-184. ISSN: 1941-0050. DOI: 10.1109/TII.2013.2262281 (cit. on p. 17).

[23] Á. Cuenca et al. "Periodic Event-Triggered Sampling and Dual-Rate Control for a Wireless Networked Control System With Applications to UAVs". In: IEEE Transactions on Industrial Electronics 66.4 (Apr. 2019), pp. 3157-3166. ISSN: 0278-0046. DOI: 10.1109/TIE. 2018.2850018 (cit. on p. 17). 
[24] Daniel Georgiev and Dawn M. Tilbury. "Packet-based control: The H2optimal solution". In: Automatica 42.1 (2006), pp. 137-144. ISSN: 00051098. DOI: https://doi .org/10.1016/j . automatica . 2005.08.011 (cit. on p. 17).

[25] Yun-Bo Zhao, Guo-Ping Liu, and David Rees. "Design of a Packet-Based Control Framework for Networked Control Systems". In: IEEE Transactions on Control Systems Technology 17.4 (July 2009), pp. 859-865. ISSN: 1063-6536. DOI: 10.1109/TCST. 2008.2010946 (cit. on p. 17).

[26] Yun-Bo Zhao, Guo-Ping Liu, and David Rees. "Modeling and Stabilization of Continuous-Time Packet-Based Networked Control Systems". In: IEEE Transactions on Systems, Man, and Cybernetics, Part B (Cybernetics) 39.6 (Dec. 2009), pp. 1646-652. ISSN: 1083-4419. DOI: 10.1109/ TSMCB. 2009. 2027714 (cit. on p. 17).

[27] Yun-Bo Zhao et al. "Packet-Based Control Design for Networked Control Systems". In: Packet-Based Control for Networked Control Systems. Springer, 2018, pp. 15-32 (cit. on p. 17).

[28] J. Alcaina et al. "Delay-independent dual-rate PID controller for a packetbased networked control system". In: Information Sciences 484 (2019), pp. 27-43. ISSN: 0020-0255. DOI: https ://doi .org/10.1016/j.ins . 2019.01.059 (cit. on pp. 17, 19).

[29] Ángel Cuenca et al. "A Remote Control Strategy for an Autonomous Vehicle with Slow Sensor Using Kalman Filtering and Dual-Rate Control". In: Sensors 19.2983 (13 2019). DOI: https://doi.org/10.3390/ s19132983 (cit. on pp. 17, 19).

[30] José Alcaina et al. "Energy-efficient control for an unmanned ground vehicle in a wireless sensor network". In: Journal of Sensors 2019 (2019). DOI: https://doi.org/10.1155/2019/7085915 (cit. on pp. 17-19).

[31] Luigi Fortuna et al. Soft Sensors for Monitoring and Control of Industrial Processes. Jan. 2007. ISBN: 978-1-84628-479-3. DOI: 10 . 1007/978-184628-480-9 (cit. on p. 17).

[32] Jay H Lee and Manfred Morari. "Robust inferential control of multi-rate sampled-data systems". In: Chemical Engineering Science 47.4 (1992), pp. 865-885 (cit. on p. 17).

[33] Dongguang Li, Sirish L Shah, and Tongwen Chen. "Analysis of dual-rate inferential control systems". In: Automatica 38.6 (2002), pp. 1053-1059 (cit. on pp. 17, 18). 
[34] Julián Salt and Pedro Albertos. "Model-based multirate controllers design". In: IEEE Transactions on Control Systems Technology 13.6 (Nov. 2005), pp. 988-997. ISSN: 1063-6536. DOI: 10.1109/TCST . 2005. 857410 (cit. on pp. 17, 18).

[35] Isaac Horowitz. "Quantitative feedback theory". In: IEE Proceedings DControl Theory and Applications. Vol. 129. 6. IET. 1982, pp. 215-226 (cit. on p. 18).

[36] Oded Yaniv. Quantitative feedback design of linear and nonlinear control systems. Springer Science \& Business Media, 1999 (cit. on p. 18).

[37] Eloy Garcia and Panos J Antsaklis. "Model-Based Event-Triggered Control for Systems With Quantization and Time-Varying Network Delays". In: IEEE Transactions on Automatic Control 58.2 (Feb. 2013), pp. 422434. ISSN: 0018-9286. DOI: 10.1109/TAC.2012.2211411 (cit. on p. 18).

[38] Songlin Hu and Dong Yue. "Event-triggered control design of linear networked systems with quantizations". In: ISA Transactions 51.1 (2012), pp. 153-162. ISSN: 0019-0578. DOI: https : // doi .org/10.1016/j . isatra.2011.09.002 (cit. on p. 18).

[39] Xiaohua Ge and Qing-Long Han. "Distributed event-triggered $H_{\infty}$ filtering over sensor networks with communication delays". In: Information Sciences 291 (2015), pp. 128-142. ISSN: 0020-0255. DOI: https: //doi.org/10.1016/j.ins.2014.08.047 (cit. on p. 18).

[40] Xiaohua Ge, Qing-Long Han, and Zidong Wang. "A threshold-parameterdependent approach to designing distributed event-triggered $H_{\infty}$ consensus filters over sensor networks". In: IEEE Trans. Cybern. (2018). DOI: 10.1109/TCYB.2017.2789296 (cit. on p. 18).

[41] Q. Liu et al. "Event-Based Recursive Distributed Filtering Over Wireless Sensor Networks". In: IEEE Transactions on Automatic Control 60.9 (Sept. 2015), pp. 2470-2475. ISSN: 0018-9286. DOI: 10.1109/TAC. 2015. 2390554 (cit. on p. 18).

[42] X. Ge, Q. Han, and Z. Wang. "A Dynamic Event-Triggered Transmission Scheme for Distributed Set-Membership Estimation Over Wireless Sensor Networks". In: IEEE Transactions on Cybernetics 49.1 (Jan. 2019), pp. 171-183. ISSN: 2168-2275. DOI: 10.1109/TCYB.2017.2769722 (cit. on p. 18).

[43] Vinh Hao Nguyen and Young Soo Suh. "Networked Estimation for EventBased Sampling Systems with Packet Dropouts". In: Sensors 9.4 (Apr. 2009), pp. 3078-3089. ISSN: 1424-8220. DOI: 10.3390/s90403078 (cit. on p. 18). 
[44] B. Sinopoli et al. "Kalman filtering with intermittent observations". In: IEEE Transactions on Automatic Control 49.9 (Sept. 2004), pp. 14531464. ISSN: 2334-3303. DOI: 10.1109/TAC. 2004.834121 (cit. on p. 18).

[45] Joris Sijs and Mircea Lazar. "On event based state estimation". In: International Workshop on Hybrid Systems: Computation and Control. Springer. 2009, pp. 336-350 (cit. on p. 18).

[46] Roy McCann and Anh T Le. "Lebesgue Sampling with a Kalman Filter in Wireless Sensors for Smart Appliance Networks". In: 2008 IEEE Industry Applications Society Annual Meeting. Oct. 2008, pp. 1-5. DOI: 10.1109/ 08IAS. 2008.9 (cit. on p. 18).

[47] S. Trimpe and R. D'Andrea. "Event-Based State Estimation With VarianceBased Triggering". In: IEEE Transactions on Automatic Control 59.12 (Dec. 2014), pp. 3266-3281. ISSN: 0018-9286. DOI: 10.1109/TAC. 2014. 2351951 (cit. on p. 18).

[48] Julián Salt et al. "A Multirate Control Strategy to the Slow Sensors Problem: An Interactive Simulation Tool for Controller Assisted Design". In: Sensors 14.3 (2014), pp. 4086-4110. ISSN: 1424-8220. DOI: 10.3390/ s140304086 (cit. on p. 18).

[49] Wei Chen and Li Qiu. "Stabilization of networked control systems with multirate sampling". In: Automatica 49.6 (2013), pp. 1528-1537. ISSN: 0005-1098. DOI: https : //doi .org/10.1016/j . automatica. 2013.02 . 010 (cit. on p. 18).

[50] Ángel Cuenca, Julián Salt, and Pedro Albertos. "Implementation of Algebraic Controllers for Non-Conventional sampled Data Systems". In: Real Time Systems 35 (2007). ISSN: 0922-6443. DOI: 10.1007/s11241-0069001-2 (cit. on p. 18).

[51] Sanjay Lall and Geir Dullerud. "An LMI solution to the robust synthesis problem for multi-rate sampled-data systems". In: Automatica 37.12 (2001), pp. 1909-1922. ISSN: 0005-1098. DOI: https://doi .org/10 . 1016/S0005-1098(01)00167-4 (cit. on p. 18).

[52] M. Araki. "Recent Developments in Digital Control Theory". In: IFAC Proceedings Volumes 26.2, Part 5 (1993). 12th Triennal Wold Congress of the International Federation of Automatic control. Volume 5 Associated Technologies and Recent Developments, Sydney, Australia, 18-23 July, pp. 951-960. ISSN: 1474-6670. DOI: https://doi.org/10.1016/S14746670(17) 48411-4 (cit. on p. 18). 
[53] Ángel. Cuenca et al. "A packet-based dual-rate PID control strategy for a slow-rate sensing Networked Control System". In: ISA Transactions 76 (2018), pp. 155-166. ISSN: 0019-0578. DOI: https://doi.org/10.1016/ j.isatra.2018.02.022 (cit. on p. 19). 



\title{
Chapter 2
}

\section{Paper 1. Control of the rotary inverted pen- dulum through threshold-based communica- tion}

\author{
Vicente Casanova, José Alcaina, Julián Salt, Ricardo \\ Pizá, Ángel Cuenca
}

This paper deals with the real implementation of an event-based control structure for the classical rotary inverted pendulum. The communication between controller and plant is performed through Ethernet (TCP/IP) which leads to a Networked Control System. The bandwidth used by the control loop is reduced, compared with the one that needs a conventional control, by using a threshold-based communication. The values of the thresholds have been determined by means of simulation techniques. The results over the real plant show how this technique can reach a significant reduction of the bandwidth consumed with a negligible worsening of the performance.

Keywords: Event-based control systems; networked control systems; bandwidth saving.

ISA Transactions, volume 62, may 2016, pages 357 - 366 https: //doi.org/10.1016/j. isatra.2016.01.009

\section{$2.1 \quad$ Introduction}

When dealing with a Networked Control System (NCS) the most valuable resource is the bandwidth. In this kind of control systems, the necessary communication between the control device and the controlled plant is carried out through a shared communication medium [1-7]. A potentially large number 
of devices are using the same medium to transmit information for different purposes: control loops, monitoring, supervising, alarms, maintenance and even transmission of information not related with control tasks (real and nonreal time data).

Depending on the physical medium used to transmit the information (electrical, optical, wireless, infrared...) and on the distance between emitter and receiver devices, a certain bandwidth is available [8-11]. This must be understood as the amount of information that can be transmitted per time unit. The bandwidth must be shared out between all the information generators (writer devices) attached to the medium. When the number of sharing devices increases, the real bandwidth available to each writer decreases.

In a typical control loop there are two devices able to generate information to be sent through the shared medium: sensor and controller. There are also two information consumers (reader devices): controller and actuator. The information generated by the sensor (output samples) is consumed by the controller. The information generated by the controller (control actions) is consumed by the actuator. Two links must be implemented: sensor-to-controller (SC) and controller-to-actuator (CA). In a conventional control system each link is implemented through its own physical medium but in a NCS they are virtual links implemented through the same medium. If a physical medium offers a bandwidth $B$ bps (bits-per-second) and L control loops are going to be closed through it, each writer has a real bandwidth of $B /(2 * L)$ bps. Of course this happens when all the writers have the same priority and the bandwidth is assigned no matter if it is needed or not. This is the policy when all the control loops are using the same sample time and the same number of samples/actions per second is transmitted. It is also possible that different writers have different needs and they get a different bandwidth. Loops with smaller sample time get a larger portion of the shared bandwidth. In this case the bandwidth is assigned to each writer depending on the control frequency and is constant during its operating life. This pre-assigned bandwidth policy is often used in industrial communication media as, for example, Profibus-DP [12-14].

But as any control engineer knows, the need for information transmission depends on the state of the controlled plant. There are periods of time in which the system is quite stable. There are neither reference changes nor perturbations and the controlled signal (i.e. the plant output) remains (almost) in the same value. Along these periods the assigned bandwidth is wasted transmitting (almost) the same sample and (almost) the same control action. To optimize the use of the precious resource an 'on-demand' policy appears to be appropriated. The main idea is to send only those sample/actions which are 
significant enough according to the control system behavior. The information not sent (because it has been considered negligible) leave the resource free to be used by other device. Obviously, this strategy needs a communication medium in which the bandwidth is not pre-assigned but is requested by the writer device. The goal is to use the minimum bandwidth to get the desired behavior for the controlled plant. TCP/IP follows this 'on-demand' policy and it is the communication protocol to be used in this work [15-18].

This event-triggered control strategy [19-27] differs from conventional control in the fact that the sample period is not determined only by the plant dynamics and the design specifications. The effective sample time (which determines the consumed bandwidth) is variable depending on the state of the controlled plant. When the plant is in a stable situation (the reference to be followed is not changing and there are no significant disturbances to cancel) the sample period increases (consuming less bandwidth) and when the plant is moving, the sample period decreases (consuming more bandwidth) towards the one used in conventional (constant sample time) control.

This can be compared with the variable simulation step in simulation theory $[28,29]$. When implementing an integration method it can be done in fixed or variable step. In fixed step the simulation step is constant during the life of the simulation. Variable step (frequently used as default in general purpose simulation tools) means that the simulation step increases or decreases depending on the computation needs to reach good quality results, according to a certain tolerance. In fixed step the number of simulation steps (and so, the computational cost) is constant and known and it is independent on the simulated system behavior. In variable step computational cost depends on the system dynamics. When simulating a system with long stable periods of time (in which the state does not change significantly) some simulation steps can be skipped (i.e. saved) to reduce the computational cost and to finish the simulation earlier. These variable step simulation based techniques are going to be applied in this work to a classical control problem. They will reduce, in a significant way, the need of bandwidth without degrading the system performance. Instead of saving computational cost the technique is used here to save bandwidth.

In variable step simulation the decision of changing the simulation step (i.e. computing or not each step) is based on an estimation of the simulation error. Two different integration methods are used and the results are compared. If the difference is below a certain tolerance it is assumed that the simulation is performing well and the step is increased, saving computational cost. In the control strategy proposed in this paper the condition to change the effective 
sampling period (i.e. transmitting or not each sample/action) is slightly different. If the difference between one sample/action and the previous one is below a certain threshold, the sample is not transmitted, saving bandwidth for other devices. This threshold must be chosen not too small to get a significant reduction on the used bandwidth and not too large to avoid a significant worsening of the system performance. And this is the main challenge: to choose the appropriated threshold to get a good bandwidth/performance ratio.

The threshold is the parameter to decide if the difference between one sample/action and the previously transmitted one is negligible or not. This parameter must be pre-arranged to an appropriated value. It can be done experimentally working with the real plant to be controlled or with a reliable enough mathematical model. Only constant thresholds are used in this work, that is intended to be continued using variable thresholds. In variable threshold it will be dynamically adapted during the life of the control system in a similar way as it is modified the size of the step in variable step simulation.

The fact that not all the samples/actions are going to be transmitted, according to its difference with the previous one, leads to an event sampling control system. The transmission of one sample/action is not caused by an external event but by an event which depends on the plant state which is, in fact, modified by the event itself.

The aim of this work is to prove that the previously described threshold-based control strategy can be applied to a real plant with a significant gain on the bandwidth consumption. In conventional control the easy way to save bandwidth is to increase the sampling period (i.e. to decrease the control frequency). This is simple and efficient but only useful when the sampling time requirements of the controlled plant are low. When controlling a plant with small time constants, the lower bound of the control frequency is easily achieved. In this case, decreasing the control frequency will lead to performance degradation and instability. The proposed strategy only makes sense if it is better than the conventional solution so, it must be tested in rough conditions, in the limits of the control frequency. The plant to be controlled will be the rotary inverted pendulum (RIP) for several reasons:

- RIP is a classical control problem frequently used as a benchmark to measure the goodness of control structures.

- RIP is an unstable and non-minimum phase plant, with a small time constant, which becomes easily unstable when the control frequency is decreased. 
- RIP is a multivariable plant with (at least) three signals to transmit (two output signals and one control action). The bandwidth gain will be larger when more information is transmitted between controller and plant.

- RIP is available at our laboratory and the proposed control strategy can be implemented in real conditions not only over the simulation model.

The paper is organized as follows. section 2.2 shows the behavior of the control in ideal conditions; conventional control and small enough control frequency to reach the desired behavior. section 2.3 shows the bandwidth saving when using threshold-based communications. section 2.4 presents conclusions of this paper and future work.

\subsection{Conventional control of the rotary inverted pendulum}

The well-known rotational inverted pendulum (RIP), also named Furuta pendulum, has a DC motor that moves an arm attached to its shaft. At the end of this arm there is a joint in which it is attached the pendulum [30-35]. The goal is to keep the pendulum in its unstable equilibrium upwards position, while a certain reference signal is followed by the motor arm. In this work, the RIP developed by Quanser Consulting Inc. is going to be used. Figure 2.1 shows the plant, in the upwards position.

The results in this section have been obtained with the real RIP when using a conventional control, without bandwidth limitations. This will be the nominal behavior to compare with the one that will be achieved when reducing the amount of information transmitted between the plant and the control structure.

RIP is a continuous underactuated plant with one input, the control action applied to the DC motor carrying the pendulum $(u(t)$, measured in volts) and two outputs:

- $\theta(t)$ : Angular position of the motor shaft around the vertical axis.

- $\alpha(t)$ : Angular position of the pendulum rod around the motor shaft axis.

The theoretical multivariable model includes the first derivative of these signals (velocity) but is not going to be considered as the real plant does not have sensors to measure them. Figure 2.2 shows a schematic representation (top and front view) of RIP. 


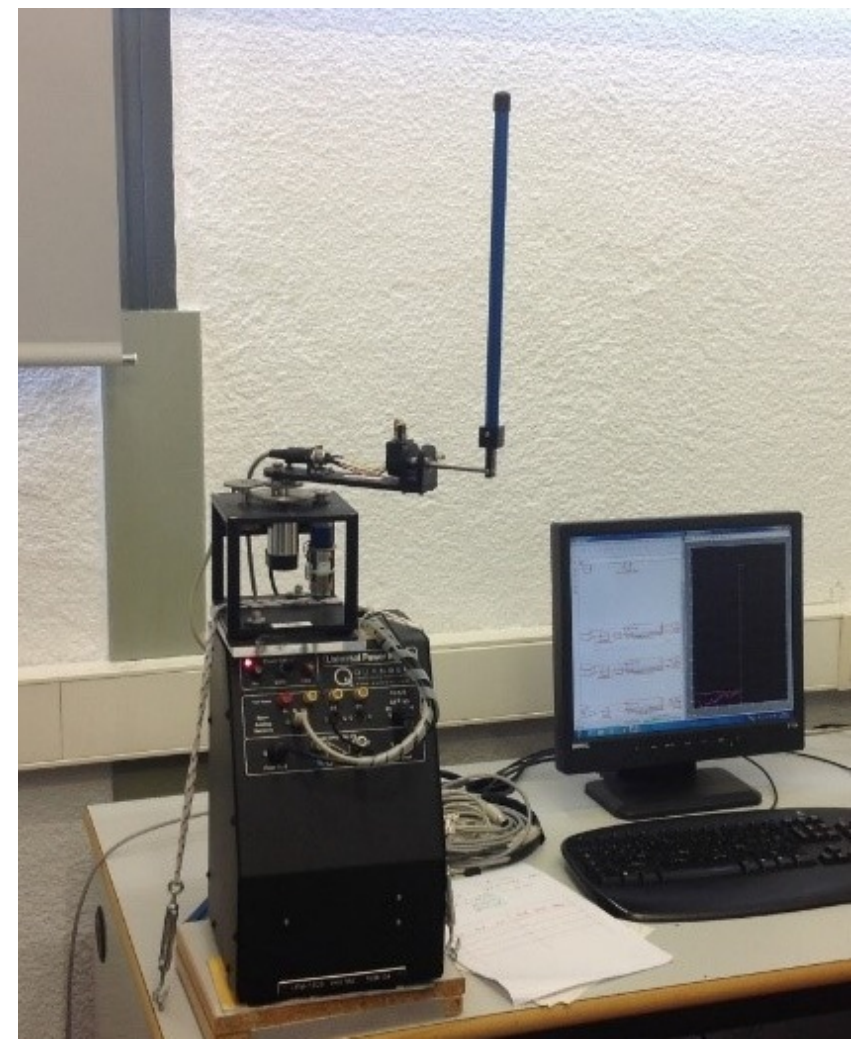

Figure 2.1: Quanser rotary inverted pendulum

The aim of this work is to prove, over a real plant, that threshold-based communication can reduce the amount of information between controller and plant without a significant worsening of the system behavior. No new controller is going to be designed to reduce the bandwidth. The controller will be the one developed to operate in a conventional control structure without bandwidth limitations. Quanser provides a LQR controller suitable to be used in ideal conditions. The controller uses as inputs $\theta(t)$ and $\alpha(t)$ (measured in the real plant), its derivatives (estimated by discrete-time derivators) and the integral of $\theta(t)$ (estimated by a discrete time integrator). The integral of $\theta(t)$ has been added to the original state vector of the plant to improve the robustness and to allow that the motor shaft can follow step references with zero error, even with the small dead zone present in the real plant. The augmented five-state vector of the plant used to design the $\mathrm{LQR}$ is: 


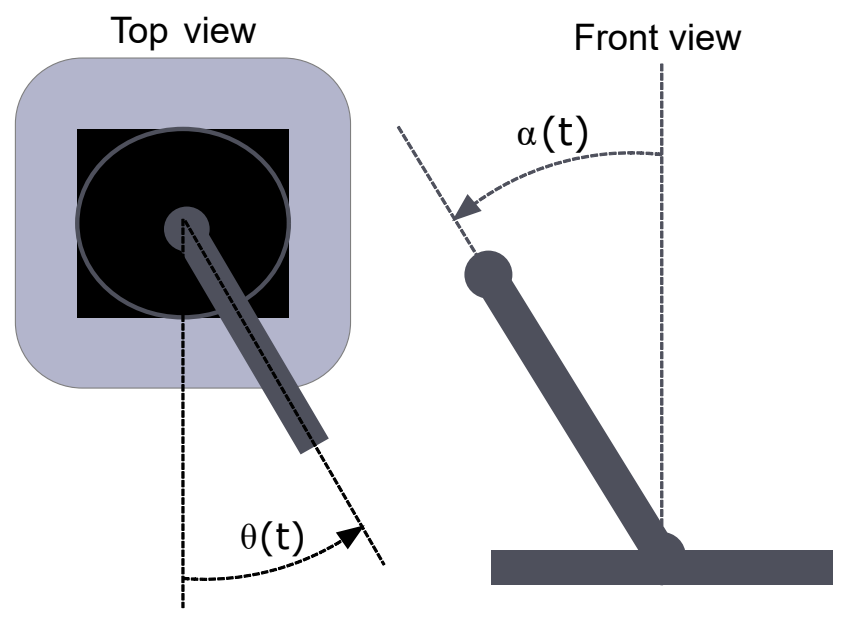

Figure 2.2: Top and front view

$$
\widetilde{X}=\left[\theta(t), \alpha(t), \frac{d \theta(t)}{d t}, \frac{d \alpha(t)}{d t}, \int \theta(t) d t\right]^{T}
$$

From this five-state vector, Quanser provides the gain vector for the LQR and using these gains, the control action to be applied to the plant can be calculated by the control structure as follows:

$$
\begin{aligned}
U & =K \cdot \widetilde{X}=\left[k_{1}, k_{2}, k_{3}, k_{4}, k_{5}\right] \cdot \widetilde{X}= \\
& =k_{1} \cdot \theta(t)+k_{2} \cdot \alpha(t)+k_{3} \cdot \frac{d \theta(t)}{d t}+k_{4} \cdot \frac{d \alpha(t)}{d t}+k_{5} \cdot \int \theta(t) d t= \\
& =\left(k_{1} \cdot \theta(t)+k_{3} \cdot \frac{d \theta(t)}{d t}+k_{5} \cdot \int \theta(t) d t\right)+\left(k_{2} \cdot \alpha(t)+k_{4} \cdot \frac{d \alpha(t)}{d t}\right)
\end{aligned}
$$

As it can be seen in the previous expression, the LQR controller has been divided in two independent sub-controllers for a better manipulation of the feedback signals. These two sub-controllers become a PID one for $\theta(t)$ and a PD controller for $\alpha(t)$. The parameters of these PID and PD controller, in the standard industrial form are: 


$$
\begin{array}{lll}
K p_{\theta}=k_{1} & T d_{\theta}=k_{3} / k_{1} & T i_{\theta}=k_{1} / k_{5} \\
K p_{\alpha}=k_{2} & T d_{\theta}=k_{4} / k_{2} & T i_{\theta}=\infty
\end{array}
$$

The discretized version of these controllers are the ones used to obtain the results presents in this work. The addition of the two outputs of these subcontrollers becomes the control action to be applied to the plant. The resulting control structure is shown in Figure 2.3. Details on this control structure can be found in [9], referred to the double rotary inverted pendulum.

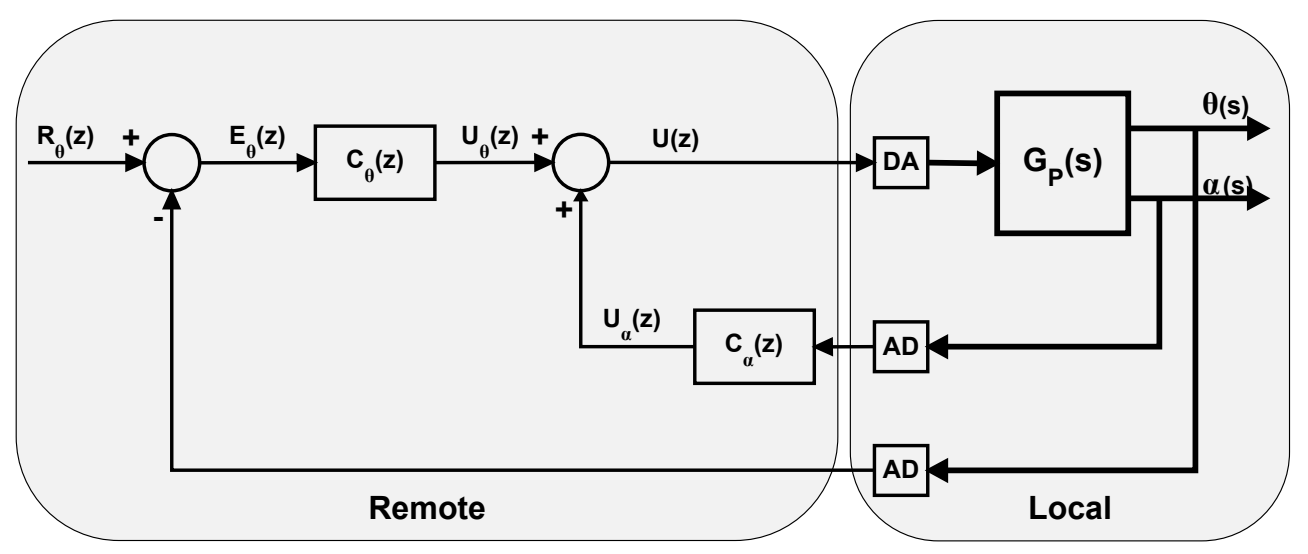

Figure 2.3: Control structure in ideal conditions

The control structure must use a small enough sample period $(T)$ to reach a good behavior. Working with the real plant it has been stated $T=10 \mathrm{~ms}$. as the most appropriated sample period to be used. Larger periods are not able to keep the plant stable while following the reference in an appropriated way. Smaller periods mean an unnecessary large need of bandwidth and computational cost. So, $T=10 \mathrm{~ms}$. has been found as the period to use in conventional control to reach a satisfactory behavior with the minimum amount of information transmitted between controller and plant. With this sample period, a bandwidth of 100 samples/second is consumed in each signal. As there are three transmitted signals, the control in ideal conditions needs a total bandwidth of 300 samples/second (200 in SC link and 100 in the CA link). This will be the nominal value of the consumed bandwidth $\left(B W_{0}\right)$ used for comparison with the one consumed when the threshold-based communication will be implemented. 
The results these conditions are shown in Figure 2.4. Both controllers have been implemented in one computer (the remote side of the NCS). A/D and D/A converters have been placed in a different computer (the local side of the NCS). Ethernet (TCP/IP) has been used as the communication medium to implement $\mathrm{SC}$ and $\mathrm{CA}$ links. The distance between remote and local computer in the experiments is short enough to consider negligible the transmission delays. No information is lost during the communication.
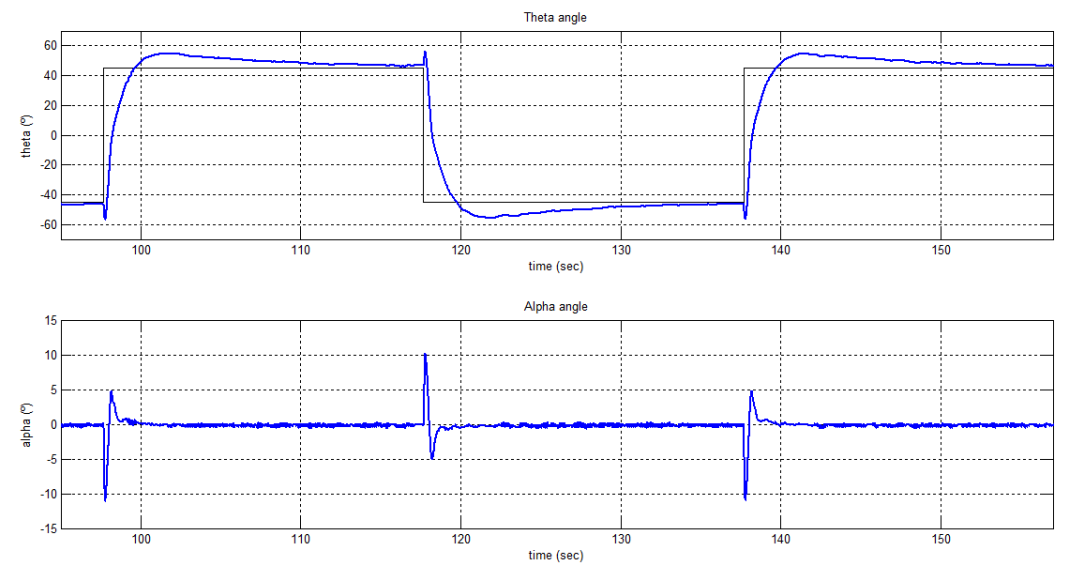

Figure 2.4: Theta and alpha angles: $\mathrm{T}=10 \mathrm{~ms}$

A square wave of $\pm 45^{\circ}$ amplitude has been used as reference signal to be followed by $\theta(t)$. Upper graphic shows $\theta(t)$ versus reference and lower one shows $\alpha(t)$. As can be seen, conventional control makes the motor shaft to follow the desired reference while keeps the angle of the pendulum rod around $0^{\circ}$, compensating the unavoidable mechanical disturbances. This nominal behavior is not going to be improved in this work. The goal is to keep the system as close as possible to this behavior while reducing the bandwidth used to feedback the controlled signals.

Conventional solution to reduce bandwidth is to increase the sample period. Using $T=15 \mathrm{~ms}$. (33\% bandwidth reduction) the behavior is significantly worse, as shown in Figure 2.5. Using a $T=20 \mathrm{~ms}$, ( $50 \%$ bandwidth reduction) the plant becomes completely unstable. As will be shown in the following section, threshold-based communication will get a significant reduction of the bandwidth without a significant worsening of the system behavior. 

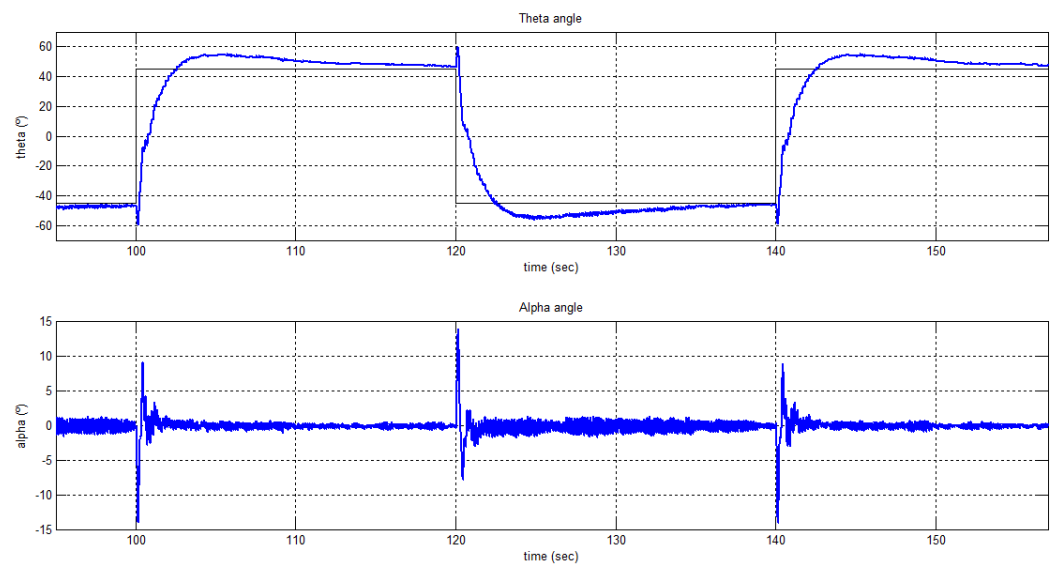

Figure 2.5: Theta and alpha angles: $\mathrm{T}=15 \mathrm{~ms}$

To measure the worsening of the system behavior a quality index is needed. The quality measure used in this work involves the integral of the unsigned tracking error (i.e. the difference between reference and angular shaft position) and the integral of the unsigned pendulum angle:

$$
E R=\int_{0}^{t}|r(\tau)-\theta(\tau)| d \tau+\int_{0}^{t}|\alpha(\tau)| d \tau
$$

Using the results with conventional control the nominal value of this error measure has been calculated. Comparing this nominal error $\left(E R_{0}\right)$ with the one obtained with threshold-based communication the system worsening can be quantitatively measured.

\subsection{Threshold-based control of the rotary inverted pendulum}

Threshold-based communication is implemented to reduce the amount of information transmitted through the shared communication medium. In the RIP control loop there are three discrete signals that must be transmitted between controller and plant. So, there are three data to be sent in every sample period: the samples of the two controlled signals captured by the sensors (i.e. the shaft and pendulum angles) and the control action generated by the controller and to be applied by the actuator. The three information generators (sensors and 
controller) are time-based devices. The clock to determine the generation of samples and control actions works with the period used in ideal conditions: $T=10 \mathrm{~ms}$. As it has been said, with a conventional time-based control 300 samples per second must use the link between controller and plant. Using an event-based control policy the exchange of information is not necessary to be equal spaced in time. The main idea is to transmit only the significant information, leaving the shared medium idle to be used for a different purpose when the information is negligible. To reach this goal, it must be determined by some means which samples/actions are significant and which ones are negligible, according to the system stability and performance.

The implementation in this work uses thresholds to determine the significance of every sample/action to be sent through the shared medium. Each sample captured by the sensors and each action generated by the controller is compared with the previously transmitted one. If the difference between one generated data and the last significant one is below a certain pre-arranged threshold is considered negligible. In this case the sample/action it is not transmitted to the other side of the shared communication medium. Note that the comparison is between the new sample/action and the last non-negligible one, not the previous captured/generated one. This must be done this way because if not, small differences between samples/actions can become an important difference with the last transmitted sample/action. Being $y_{k}$ the information captured/generated in a given sample instant and TRH the value of the threshold, the transmitted information, $\hat{y}_{k}$, will be:

$$
\hat{y}_{k}=\left\{\begin{array}{lll}
\hat{y}_{k-1} & \text { when }\left|y_{k}-\hat{y}_{k-1}\right| \leqslant T R H & \text { (not transmitted) } \\
y_{k} & \text { when }\left|y_{k}-\hat{y}_{k-1}\right|>T R H & \text { (transmitted) }
\end{array}\right.
$$

Note that when $\hat{y}_{k}=\hat{y}_{k-1}$ the new sample action is not transmitted. Data not transmitted leave the medium idle to be used by some other device, saving bandwidth for a different purpose. The main idea is not to waste bandwidth when the new data does not provide significant information. The algorithm implemented between the information generators and the shared medium is depicted in Figure 2.6.

The key point in this communication policy is the value of the threshold. A too small threshold will cause that too many data will be sent and there will not be a significant bandwidth saving. If the threshold is too large significant information will be lost, leading to performance degradation and even instability. In addition, if the threshold is too large, the degradation of the 


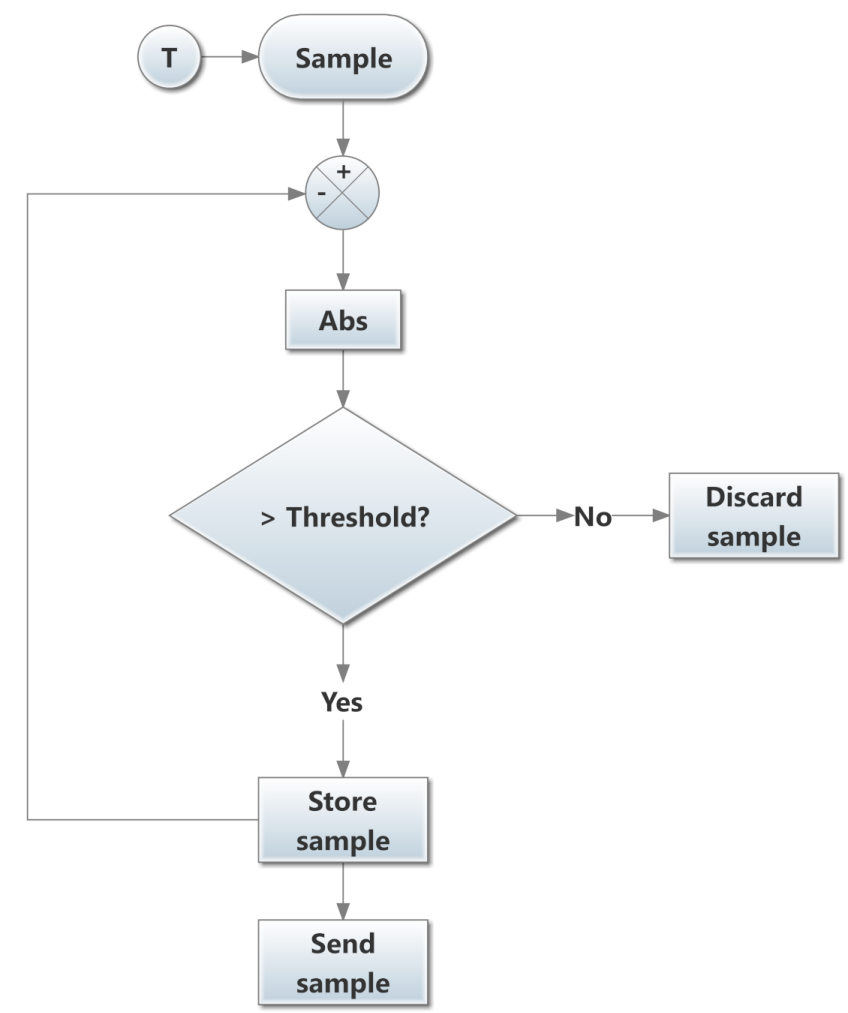

Figure 2.6: Threshold-based communication flowchart

system performance will cause larger changes in the data to be sent. In this case the bandwidth is only not reduced but can be even increased. There must be a compromise between the reduction of the consumed bandwidth and the increasing of the error index.

The most appropriated value for the thresholds are going to be determined by means of simulation. The values will be chosen to reach a good error/bandwidth ratio, measured by a certain index. In this work, the procedure to determine the thresholds will be applied to the RIP control but can be applied to any other control problem to reduce the consumed bandwidth. The only requirement to apply the procedure described in this work is to have a good simulation model of the controlled plant. This model can include any kind of non-linear 
behavior, disturbances and uncertainties for a better representation of the real plant.

\subsubsection{Optimal threshold values determination}

There is not an easy way to determine the optimal values of the thresholds. These values depends on the dynamics of the control system which depends itself on the thresholds. But if a good mathematical model of the controlled plant is available they can be calculated by means of simulation. This will not lead to the optimal thresholds but to the best among all the values tested in the simulation study. The procedure is as follows:

- The behavior of the control system using certain values for the thresholds is simulated.

- The quality of the response is measured calculating the error index $(E R)$.

- The consumed bandwidth $(B W)$ is measured counting the number of samples/actions transmitted during the simulation.

Repeating the procedure for a large enough collection of possible values for the thresholds, the most appropriated values for the three thresholds have been determined. To reach these values a large number of trials have been done varying the thresholds and measuring the error index and the consumed bandwidth.

The ratio between the error index and the one obtained with conventional control $\left(E R_{0}\right)$ provides the relative error $\left(E R_{R}\right)$, always above one. The larger this relative error is, the worse behavior is caused.

$$
E R_{R}=E R / E R_{0}
$$

The ratio between the number of data above the threshold and the consumed bandwidth with time-based control $\left(B W_{0}\right)$ is the relative bandwidth index $\left(B W_{R}\right)$, always below one. The smaller this relative bandwidth is, the larger bandwidth saving is gained.

$$
B W_{R}=B W / B W_{0}
$$

The results of this study are presented in Figure 2.7 (relative error and relative bandwidth versus theta threshold), Figure 2.8 (relative error index and relative 
bandwidth versus alpha threshold) and Figure 2.9 (relative error index and relative bandwidth versus control action threshold).
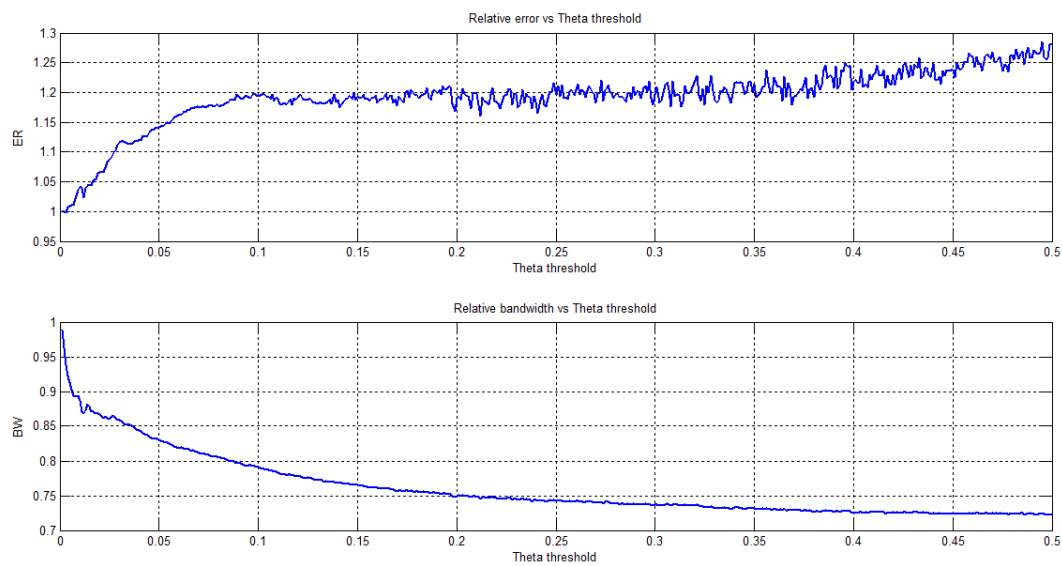

Figure 2.7: Theta threshold
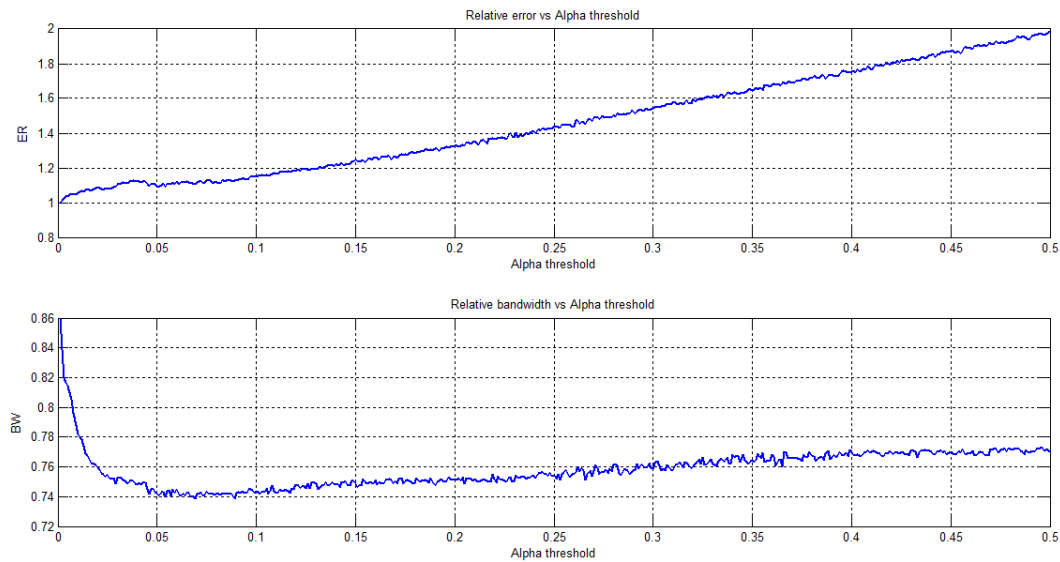

Figure 2.8: Alpha threshold

The results from this experiment show that, even if threshold-based communication is used in only one of the three signals, a significant reduction of the consumed bandwidth can be achieved without a significant worsening of the behavior. But if the threshold is increased beyond a certain point the bandwidth 

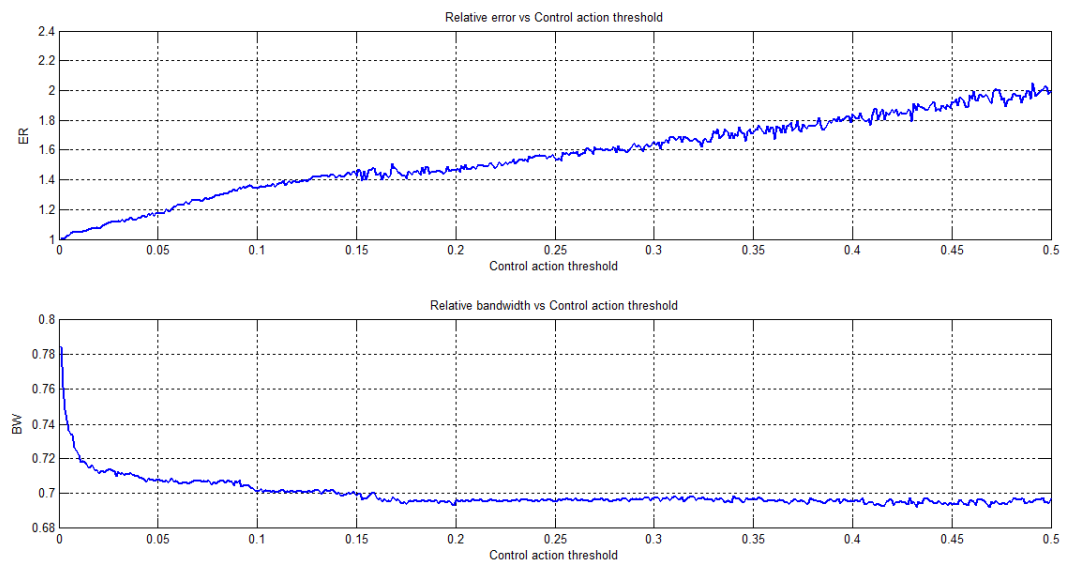

Figure 2.9: Control action threshold
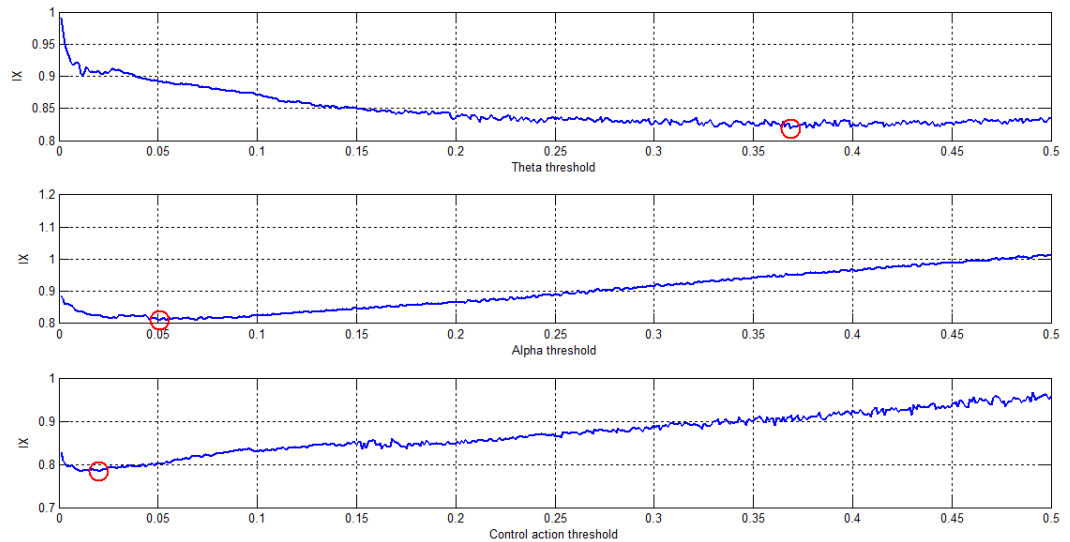

Figure 2.10: Performance index

gain is much smaller than the performance worsening. An index combining these two experimental measures must be used to decide the optimal value of the threshold. The error/bandwidth index can be calculated giving the appropriated weigh to each one. It is not an easy problem to choose this weigh because it depends on when the bandwidth improvement is less significant than the behavior worsening. After some experiments with the real plant and the 
simulation model, it has been decided that the performance index to be used in this work will be:

$$
I X=0.2 \cdot E R_{R}+0.8 \cdot B W_{R}
$$

The values of the threshold that minimize this index will be the ones to use in the real implementation. Figure 2.10 shows this performance index versus the three thresholds, considered one by one. The minimum value (marked with a circle) gives the optimal (according to the selected index) value of the thresholds.

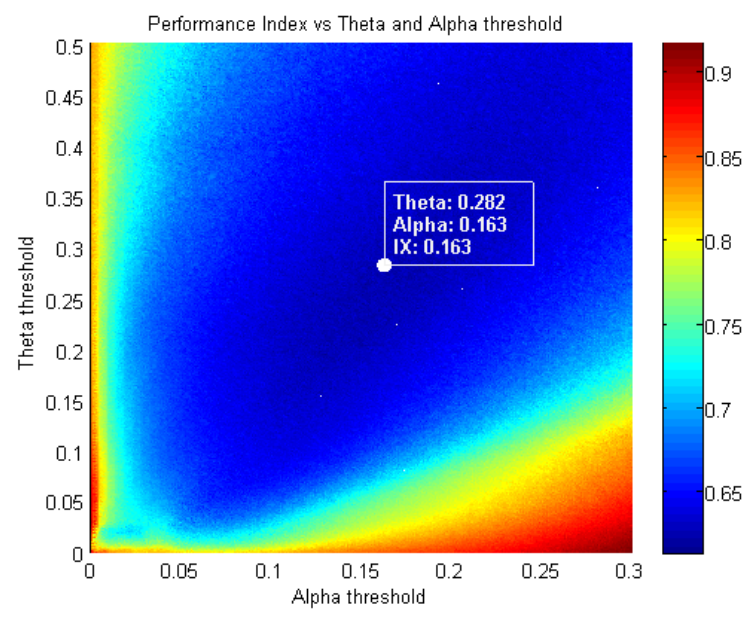

Figure 2.11: Theta and alpha thresholds

Using only one threshold an index of around 0.8 can be achieved. It is obvious that better results can be achieved if two thresholds are used together. Figures $2.11,2.12$, and 2.13 show the index $I X$ when varying a pair of thresholds. The color in these figures indicates the value of the index to be minimized. The white dot marks the minimum of the index and the values chosen for the thresholds. These results, obtained using the simulation model of the RIP, show that when using a pair of thresholds the performance index can be improved up to around 0.6 as the bandwidth is saved in two of the transmitted signals instead of only one as in the first experiment.

Finally, it is easy to think that the results can be improved by using eventbased communication for the three signals. After a (large) simulation study 


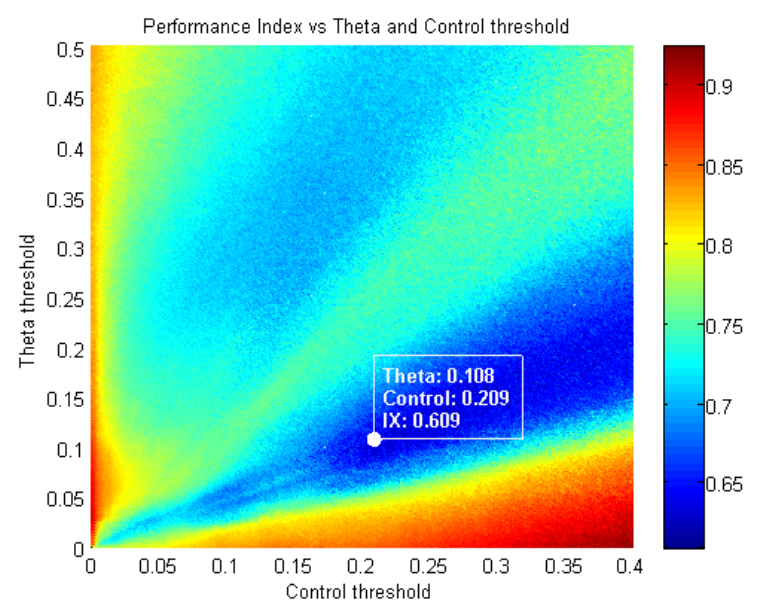

Figure 2.12: Theta and control thresholds

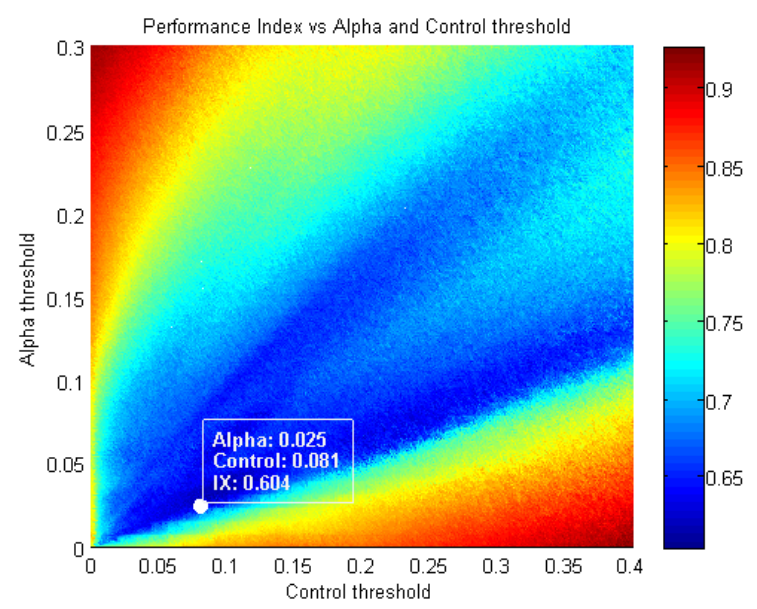

Figure 2.13: Alpha and control thresholds

with three nested loops the optimal (again, according to the chosen index) values of the thresholds have been stated as follows:

- Theta threshold: 0.36 .

- Alpha threshold: 0.29 . 
- Control threshold: 0.26 .

As there are four variables involved (the index and the three thresholds) there is no possible graphic representation of the results in this experiment but the simulation shows that using these values for the thresholds, the performance index can be reduced below 0.5 . In these conditions the error index is only $4.5 \%$ larger than the one with conventional control but the consumed bandwidth is only the $34 \%$ than the one needed with time-based communication. If these results are confirmed in the real plant, a reduction of the bandwidth around $60-70 \%$ will be achieved with a behavior that will be almost the same than the one with conventional control. Using the conventional control structure it is not possible to stabilize the RIP using such a small bandwidth.

\subsubsection{Experimental results with threshold-based control}

Using the values determined in the previous section for the thresholds, the results in the real plant are shown in Figure 2.14. Again, Ethernet (TCP/IP) has been used to send the information between controller and plant. As can be seen the behavior is slightly worse than in ideal conditions (relative error is 1.06) but the consumed bandwidth is much better (relative bandwidth is 0.31 ). This means that constant threshold has been achieved a bandwidth saving of $69 \%$, regarding to the consumed in nominal conditions.
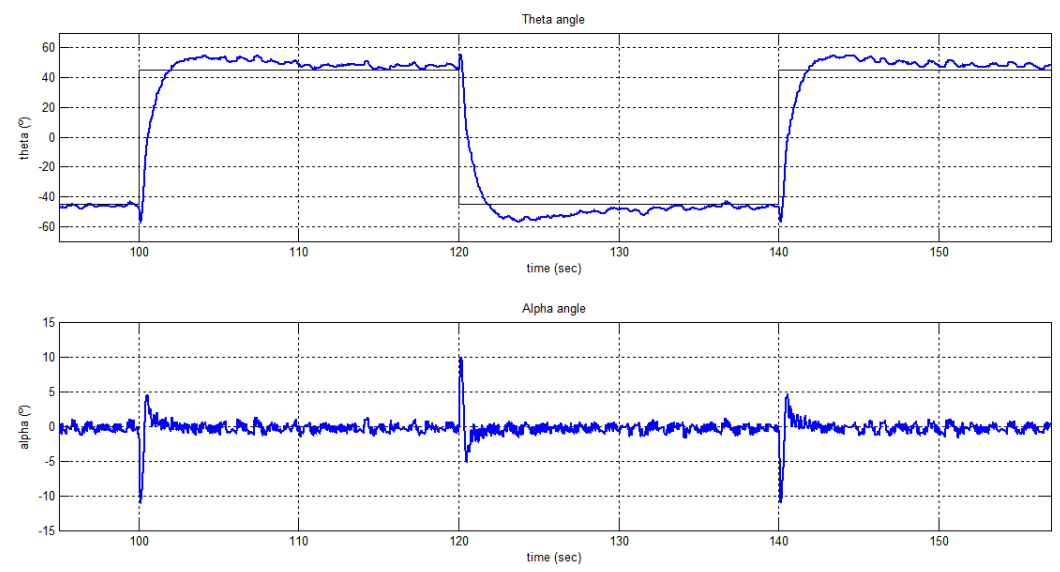

Figure 2.14: Theta and alpha: Threshold control 
Figure 2.15 shows a detail of a small piece of the theta angle. Upper plot is the signal captured by the sensor and lower one is the signal effectively transmitted to the controller. Samples marked with a circle are the ones which passes the threshold condition and are sent through the shared medium (consuming bandwidth). Samples marked with a solid circle are the ones not transmitted (saving bandwidth). Similar results could be shown in alpha and control action signals.
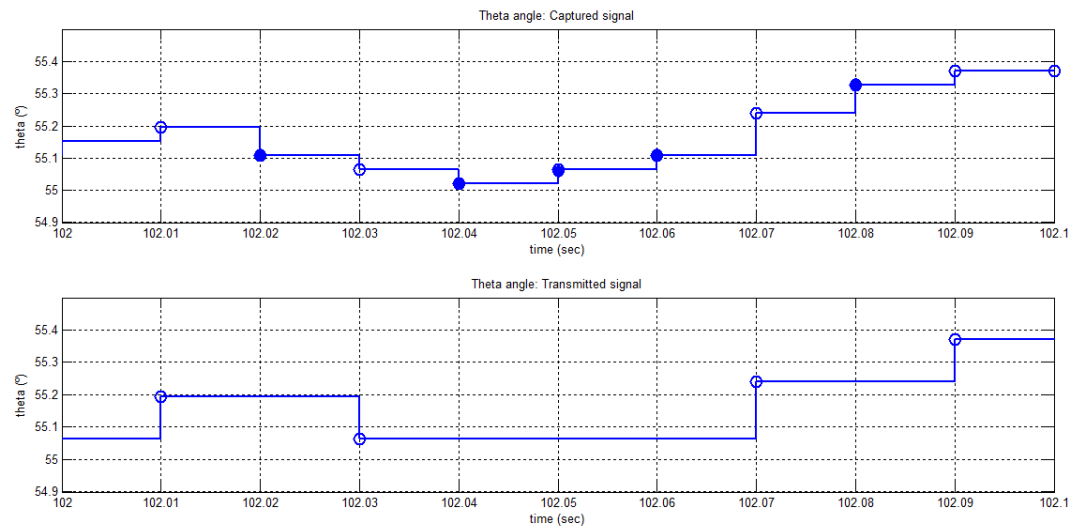

Figure 2.15: Theta angle: Captured and transmitted

To understand how this saving is achieved Figure 2.16 shows the transmission flag for the theta angle. This flag indicates when a sample is effectively transmitted through the shared medium (marked as ones) or skipped as being considered negligible (marked as zeros). The upper plot show data belonging to the transient and the lower show data belonging to the steady state. As can be seen, during the transient as the state of the plant is changing, most of the samples are transmitted. When the plant reaches the steady state, much more samples are discarded, increasing the bandwidth saving. This different need of bandwidth suggest that the values of the thresholds can be dynamically changed for a better use of the available bandwidth. Variable thresholds are proposed as an extension to this work.

The experiment has been repeated (over the real plant) using only one and two thresholds to see how different relative errors and bandwidths can be achieved. The results are summarized in Table 2.1. As expected, the more thresholds are used the best bandwidth saving and performance index are achieved. 

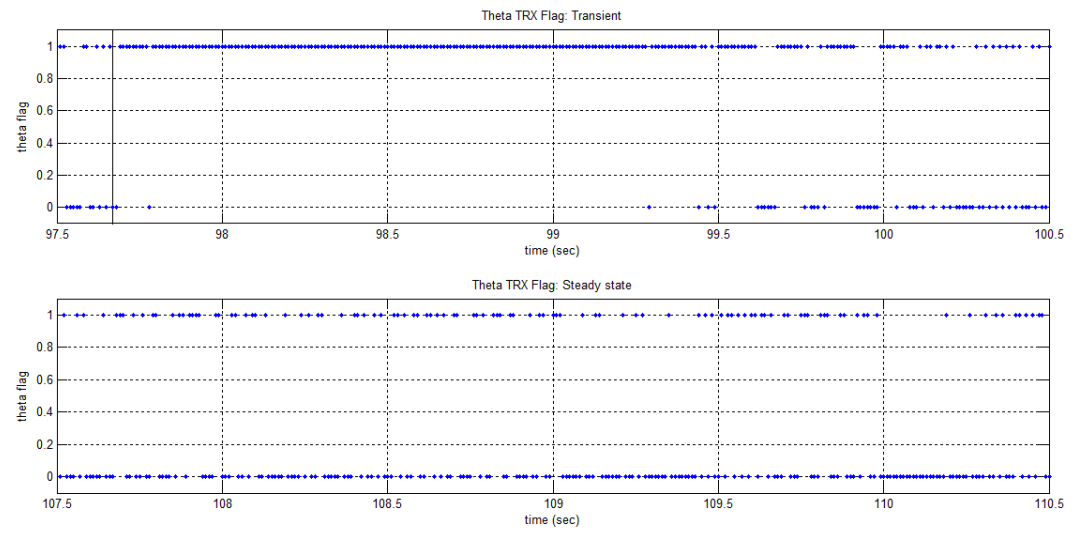

Figure 2.16: Theta transmission flag

\begin{tabular}{|l|lll|}
\hline Thresholds & $E R_{R}$ & $B W_{R}$ & $I X$ \\
\hline Theta & 1.0290 & 0.7273 & 0.7876 \\
Alpha & 1.0103 & 0.7468 & 0.7995 \\
Control & 1.0171 & 0.7803 & 0.8277 \\
Theta \& Alpha & 1.0108 & 0.4787 & 0.5851 \\
Theta \& Control & 1.0408 & 0.6270 & 0.7097 \\
Theta \& Control & 1.0279 & 0.6188 & 0.7006 \\
Theta, Alpha \& Control & 1.0614 & 0.3136 & 0.4632 \\
\hline
\end{tabular}

Table 2.1: Alpha and control action thresholds

\subsection{Conclusions and future work}

The work in this paper shows the implementation of an event-sampling control, over a real plant and using a real shared communication medium to close the loop. The goal has been to reduce the amount of information exchanged between controller and plant, without a significant loss of control performance. This bandwidth reduction has been achieved by means of using a thresholdbased communication. Rotary inverted pendulum has been used for this implementation due to its bandwidth needs and sensitivity to the sampling period. TCP/IP has been used as communication protocol because it follows an 'ondemand' policy and in this kind of sharing policy is where the bandwidth saving is effective. 
The results show how the threshold-based communication can be easily used to reduce significantly the consumed bandwidth and the behavior of the system is almost the same than the one with conventional control. Simulation model of the controlled plant has been used to select the most appropriated values for the thresholds to minimize an index, involving bandwidth and performance.

The procedure to determine the best values for the thresholds have been determined using the simulation model of the controlled plant. This procedure, applied in this work to the particular case of controlling the rotary inverted pendulum, can be exported to the control of any other plant no matter if it is linear or not, stable or not, as far as a good enough model is available.

A detailed analysis of the results when using threshold-based communication shows that the need for bandwidth is not constant during the life of the system. It depends on the state of the controlled plant which depends on the reference signal and on the presence of significant disturbances. The pre-arranged values for the thresholds has been obtained in certain environment conditions (reference signal to be followed, communication delays, signals noise, performance index, etc). If these conditions change, maybe the pre-arranged values are not the most appropriated. This suggest an adaptive, variable threshold-based communication in which the values for the thresholds are dynamically modified depending on the plant state. This adaptive policy is expected to improve the results in this paper.

In addition, when using constant thresholds the system does not reach an equilibrium position. A certain ripple around the desired value appears when the motor shaft reference is constant. The presence of this undesired characteristic it is difficult to be seen in this particular plant because the upwards position is an unstable equilibrium point and same ripple is always present, even when conventional control is applied. Using an stable plant the ripple caused by the threshold can be considered in the performance index as a new factor to measure the system behavior. Variable thresholds are expected to help in solving this problem without increasing the consumed bandwidth.

Another improvement to be considered is to use a different sample period in the threshold comparison, leading to a dual-rate structure that could improve the performance without increasing the consumed bandwidth. 


\section{Acknowledgements}

This work was supported by the Spanish Ministerio de Economía y Competitividad under Grant referenced TEC2012-31506. 


\section{References}

[1] L. G. Bushnell. "Networks and control [Guest Editorial]". In: IEEE Control Systems Magazine 21.1 (Feb. 2001), pp. 22-23. ISSN: 1066-033X. DOI: 10.1109/MCS. 2001.898789 (cit. on p. 29).

[2] Wei Zhang, M. S. Branicky, and S. M. Phillips. "Stability of networked control systems". In: IEEE Control Systems Magazine 21.1 (Feb. 2001), pp. 84-99. ISSN: 1066-033X. DOI: 10.1109/37.898794 (cit. on p. 29).

[3] Yodyium Tipsuwan and Mo-Yuen Chow. "Control methodologies in networked control systems". In: Control Engineering Practice 11.10 (2003). Special Section on Control Methods for Telecommunication, pp. 10991111. ISSN: 0967-0661. DOI: https://doi .org/10.1016/S0967-0661(03) 00036-4 (cit. on p. 29).

[4] L. A. Montestruque and P. Antsaklis. "Stability of model-based networked control systems with time-varying transmission times". In: IEEE Transactions on Automatic Control 49.9 (Sept. 2004), pp. 1562-1572. ISSN: 0018-9286. DOI: 10.1109/TAC. 2004.834107 (cit. on p. 29).

[5] J. P. Hespanha, P. Naghshtabrizi, and Y. Xu. "A Survey of Recent Results in Networked Control Systems". In: Proceedings of the IEEE 95.1 (Jan. 2007), pp. 138-162. ISSN: 0018-9219. DOI: 10.1109/JPROC. 2006.887288 (cit. on p. 29).

[6] Wei Chen and Li Qiu. "Stabilization of networked control systems with multirate sampling". In: Automatica 49.6 (2013), pp. 1528-1537. ISSN: 0005-1098. DOI: https://doi.org/10.1016/j .automatica.2013.02 . 010 (cit. on p. 29).

[7] Hoang-Dung Tran et al. "A normalized PID controller in networked control systems with varying time delays". In: ISA Transactions 52.5 (2013), pp. 592-599. ISSN: 0019-0578. DOI: https : // doi .org/10.1016/j . isatra.2013.05.005 (cit. on p. 29). 
[8] J. Baillieul and P. J. Antsaklis. "Control and Communication Challenges in Networked Real-Time Systems". In: Proceedings of the IEEE 95.1 (Jan. 2007), pp. 9-28. ISSN: 0018-9219. DOI: 10.1109/JPROC. 2006.887290 (cit. on p. 30$)$.

[9] Vicente Casanova et al. "Networked Control Systems: control structures with bandwidth limitations". In: International Journal of Systems, Control and Communications 1.3 (2009), pp. 267-296. DOI: 10.1504/I JSCC . 2009 . 024556. eprint: https : / / www . inderscienceonline .com/doi / pdf/10.1504/I JSCC. 2009.024556 (cit. on pp. 30, 36).

[10] Ming Liu, Qingling Wang, and Hongyi Li. "State estimation and stabilization for nonlinear networked control systems with limited capacity channel". In: Journal of the Franklin Institute 348.8 (2011), pp. 18691885. ISSN: 0016-0032. DOI: https://doi.org/10.1016/j.jfranklin. 2011.05 .008 (cit. on p. 30).

[11] L. Zhang, H. Gao, and O. Kaynak. "Network-Induced Constraints in Networked Control Systems - A Survey". In: IEEE Transactions on Industrial Informatics 9.1 (Feb. 2013), pp. 403-416. ISSN: 1551-3203. DOI: 10.1109/TII.2012.2219540 (cit. on p. 30).

[12] E. Tovar and F. Vasques. "Cycle time properties of the PROFIBUS timed-token protocol". In: Computer Communications 22.13 (1999), pp. 1206 1216. ISSN: 0140-3664. DOI: https ://doi .org/10.1016/S0140-3664(99) 00123-1 (cit. on p. 30).

[13] Kyung Chang Lee, Suk Lee, and Man Hyung Lee. "QoS-based remote control of networked control systems via Profibus token passing protocol". In: IEEE Transactions on Industrial Informatics 1.3 (Aug. 2005), pp. 183-191. ISSN: 1551-3203. DOI: 10.1109/TII.2005.852064 (cit. on p. 30).

[14] Max Felser. Profibus manual: a collection of information explaining Profibus networks. epubli, 2011 (cit. on p. 30).

[15] Douglas E Comer. Internetworking with TCP/IP, Vol. I: Principles, Protocols, and Architecture, 3/e. 1995 (cit. on p. 31).

[16] Feng-Li Lian, J. R. Moyne, and D. M. Tilbury. "Performance evaluation of control networks: Ethernet, ControlNet, and DeviceNet". In: IEEE Control Systems Magazine 21.1 (Feb. 2001), pp. 66-83. ISSN: 1066-033X. DOI: $10.1109 / 37.898793$ (cit. on p. 31).

[17] J. -. Decotignie. "Ethernet-Based Real-Time and Industrial Communications". In: Proceedings of the IEEE 93.6 (June 2005), pp. 1102-1117. ISSN: 0018-9219. DOI: 10.1109/JPROC.2005.849721 (cit. on p. 31). 
[18] Ángel Cuenca et al. "A Delay-Dependent Dual-Rate PID Controller Over an Ethernet Network". In: IEEE Transactions on Industrial Informatics 7.1 (Feb. 2011), pp. 18-29. ISSN: 1551-3203. DOI: 10.1109/TII . 2010. 2085007 (cit. on p. 31).

[19] W. P. M. H. Heemels, J. H. Sandee, and P. P. J. Van Den Bosch. "Analysis of event-driven controllers for linear systems". In: International Journal of Control 81.4 (2008), pp. 571-590. DOI: 10.1080/00207170701506919. eprint: https://doi.org/10.1080/00207170701506919 (cit. on p. 31).

[20] Jan Komenda and Jan H. van Schuppen. "Control of discrete-event systems with modular or distributed structure". In: Theoretical Computer Science 388.1 (2007), pp. 199-226. ISSN: 0304-3975. DOI: https://doi. org/10.1016/j.tcs.2007.07.049 (cit. on p. 31).

[21] Karl J. Aström. "Event Based Control". In: Analysis and Design of Nonlinear Control Systems: In Honor of Alberto Isidori. Ed. by Alessandro Astolfi and Lorenzo Marconi. Berlin, Heidelberg: Springer Berlin Heidelberg, 2008, pp. 127-147. ISBN: 978-3-540-74358-3. DOI: 10.1007/978-3540-74358-3_9 (cit. on p. 31).

[22] A. Eqtami, D. V. Dimarogonas, and K. J. Kyriakopoulos. "Event-triggered control for discrete-time systems". In: Proceedings of the 2010 American Control Conference. June 2010, pp. 4719-4724. DOI: 10.1109/ACC. 2010. 5531089 (cit. on p. 31).

[23] X. Wang and M. D. Lemmon. "Event-Triggering in Distributed Networked Control Systems". In: IEEE Transactions on Automatic Control 56.3 (Mar. 2011), pp. 586-601. ISSN: 0018-9286. DOI: 10.1109/TAC. 2010. 2057951 (cit. on p. 31).

[24] Songlin Hu and Dong Yue. "Event-triggered control design of linear networked systems with quantizations". In: ISA Transactions 51.1 (2012), pp. 153-162. ISSN: 0019-0578. DOI: https : // doi .org/10.1016/j . isatra.2011.09.002 (cit. on p. 31).

[25] W. P. M. H. Heemels and M. C. F. Donkers. "Model-based periodic eventtriggered control for linear systems". In: Automatica 49.3 (2013), pp. 698711. ISSN: 0005-1098. DOI: https://doi.org/10.1016/j . automatica. 2012.11 .025 (cit. on p. 31).

[26] U. Premaratne, S. K. Halgamuge, and I. M. Y. Mareels. "Event Triggered Adaptive Differential Modulation: A New Method for Traffic Reduction in Networked Control Systems". In: IEEE Transactions on Automatic Control 58.7 (July 2013), pp. 1696-1706. ISSN: 0018-9286. DOI: 10.1109/ TAC. 2013.2242571 (cit. on p. 31). 
[27] Ángel Ruiz et al. "A practical tuning methodology for event-based PI control". In: Journal of Process Control 24.1 (2014), pp. 278-295. ISSN: 0959-1524. DOI: https://doi.org/10.1016/j.jprocont.2013.11.013 (cit. on p. 31).

[28] J.D. Lambert. Numerical Methods for Ordinary Differential Systems: The Initial Value Problem. Wiley, 1991. ISBN: 9780471929901 (cit. on p. 31).

[29] François E. Cellier and Ernesto Kofman. Continuous System Simulation. Springer, 2006. ISBN: 978-0-387-30260-7 (cit. on p. 31).

[30] K. Furuta et al. "A New Inverted Pendulum Apparatus for Education". In: Proceedings of IFAC Control Education Conference 91. 1991, pp. 191196 (cit. on p. 33).

[31] Isabelle Fantoni and Rogelio Lozano. Non-linear Control for Underactuated Mechanical Systems. Advances in Pattern Recognition. SpringerVerlag London, 2002. ISBN: 978-1-4471-0177-2 (cit. on p. 33).

[32] Pedro X. La Hera et al. "New approach for swinging up the Furuta pendulum: Theory and experiments". In: Mechatronics 19.8 (2009), pp. 12401250. ISSN: 0957-4158. DOI: https://doi org/10.1016/j . mechatronics . 2009.07.005 (cit. on p. 33).

[33] V Casanova et al. "Controlling the double rotary inverted pendulum with multiple feedback delays". In: International Journal of Computers Communications \&3 Control 7.1 (2012), pp. 20-38 (cit. on p. 33).

[34] J. Aracil, J.Á. Acosta, and F. Gordillo. "A nonlinear hybrid controller for swinging-up and stabilizing the Furuta pendulum". In: Control Engineering Practice 21.8 (2013), pp. 989-993. ISSN: 0967-0661. DOI: https: //doi.org/10.1016/j.conengprac.2013.04.001 (cit. on p. 33).

[35] M. Ramírez-Neria et al. "Linear active disturbance rejection control of underactuated systems: The case of the Furuta pendulum". In: ISA Transactions 53.4 (2014). Disturbance Estimation and Mitigation, pp. 920928. ISSN: 0019-0578. DOI: https://doi.org/10.1016/j.isatra. 2013. 09.023 (cit. on p. 33). 


\title{
Chapter 3
}

\section{Paper 2. A packet-based dual-rate PID con- trol strategy for a slow-rate sensing networked control system}

\author{
Ángel Cuenca, José Alcaina, Julián Salt, Vicente \\ Casanova, Ricardo Pizá
}

This paper introduces a packet-based dual-rate control strategy to face time-varying network-induced delays, packet dropouts and packet disorder in a Networked Control System. Slow-rate sensing enables to achieve energy saving and to avoid packet disorder. Fastrate actuation makes reaching the desired control performance possible. The dual-rate PID controller is split into two parts: a slowrate PI controller located at the remote side (with no permanent communication to the plant) and a fast-rate PD controller located at the local side. The remote side also includes a prediction stage in order to generate the packet of future, estimated slow-rate control actions. These actions are sent to the local side and converted to fast-rate ones to be used when a packet does not arrive at this side due to the network-induced delay or due to occurring dropouts. The proposed control solution is able to approximately reach the nominal (no-delay, no-dropout) performance despite the existence of time-varying delays and packet dropouts. Control system stability is ensured in terms of probabilistic Linear Matrix Inequalities (LMIs). Via real-time control for a Cartesian robot, results clearly reveal the superiority of the control solution compared to a previous proposal by authors.

Keywords: Networked control systems; slow sensors; non-uniform sampling; multi-rate control; packet-based control.

ISA Transactions, volume 76, may 2018, pages 155 - 166 https: //doi.org/10.1016/j. isatra.2018.02.022 


\subsection{Introduction}

One of the main features of a Networked Control System (NCS) [1-3] is to send data via a shared communication medium in order to control a process. In the last few years, interesting applications have been reported in this field [4-7]. The present work considers a low cost distributed sensor net, which sends data to a powerful remote server (with high computation and information storage features). One of the main goals of the contribution is to achieve energy saving in this kind of set-up. Energy saving is a crucial issue, especially in batterypowered wireless sensors, having positive effects on their battery lives. As it is well known, the energy consumption in sensor networks is usually due to sensing, processing and communication operations. The last ones consume most part of this amount of energy. Therefore, in order to save energy, a good option is to reduce the data flow frequency through the net. However, a quite low transmission frequency could imply failure to fulfil control specifications, or even process instability. Different control proposals have been introduced to reduce the communication rate preserving the control performance. One of them is the packet-based approach [8-11], which enables a sequence of signals to be sent over the network simultaneously. In the same way, techniques based on sending data when some thresholds are exceeded [12-14] -i.e. event-based control-, or just considering some specific data priority [15], have been used. Another possible option is the so called "multi-rate control", which is a control technique able to assume different rates for different control loop signals [16, 17]. The present work combines multi-rate and packet-based control techniques for dealing, in addition, with some typical NCS problems such as time-varying network-induced delays, packet dropouts, and packet disorder.

The considered NCS locates the low cost sensor net, the process and the actuator (with a low computation power) in the local side, whereas the server (with high computation power and other capabilities) is situated in the remote side. Sensed values must travel through the main network from the local side to the remote side, and control signals, from the remote side to the local side. As it can be said, to reduce energy, a slow transmission frequency was assumed in both links. Therefore, slow-rate sensed values and slow-rate control actions travel through the NCS. Adopting a dual-rate control strategy, an $N$ faster control updating (being $N$ a positive integer greater than one) can be assumed at the actuator device by converting the slow-rate control signal into a fast-rate one, which enables to achieve the desired control performance. This proposal requires a special controller, actually a non-conventional controller, which is able to compute control actions at period $T$ from signals taken at period $N T$ [17]. In this work, due to the wide knowledge of PID controllers in industrial 
and academic environments, a non-conventional PID control structure is taken into account. The controller is split into two parts: a slow-rate PI controller and a fast-rate PD controller. The integral action is applied at slow rate because it usually operates at this frequency zone, and hence, it is located at the remote (server) side. The derivative actions, which are associated with faster dynamics, are applied at fast rate, and hence, the PD controller is located at the local side (inside the actuator). The basic design procedure can be looked up in $[17-19]$.

The additional difficulties caused by the shared communication medium (networkinduced delays, packet dropouts, and packet disorder) can appear depending on the network protocol used in a specific application. Regarding the delays, these are due to waiting-transmission-picking up times and are time-varying during the course of the application. As it is well-known, not compensating for the delays can imply a worsening of the control performance. This problem can be overcome by using, for example, gain-scheduling control strategies such as the one used in [20]. This proposal allows obtaining a simple delay-dependent control law to retune the parameters of the fast-rate PD controller from the round-trip time delay. As the actuator is assumed to include low computation capabilities, it is able to measure the round-trip time delay and compute the fast-rate control signal. In this way, the nominal (no-delay) control performance can be closely maintained despite actuating in a non-uniform way due to the delays (that is, the last control action computed in the previous sensor period is held until new, current control actions are applied after the delay).

Concerning the packet disorder, once again, the fact of choosing a slow sensing period NT (concretely, the sensing period will be longer than the longest round-trip time delay) is beneficial, since it can avoid this phenomenon. Obviously, the decision needs to perform some off-line experiences with usual operation conditions to detect some delay features. Sometimes, a statistical distribution of the network-induced delay is even found [21, 22]. As previously commented, despite the fact of choosing the slow sensing period in this way, dual-rate control techniques (with faster control period) enable to achieve a satisfactory performance $[17,18]$. And, once the sensing period and the rest of NCS parameters are defined, LMIs can be used to ensure control system stability.

Finally, with regard to packet dropouts, if a network protocol like UDP is assumed, this phenomenon can occur [21, 22]. In this work, a packet dropout can be derived either from an effective loss or from the expiration of a maximum waiting time. In addition, an upper-bound for consecutive packet dropouts $M$ can be established from the off-line experiences. In order to deal with up to $M$ 
possible packet losses, a prediction stage is included at the remote side, which contains a state resetting procedure $[23,24]$ in order to face even unstable processes (or marginally stable ones, such as the position output for DC motors governing the axis of a Cartesian robot, which is used in this work). If a packet dropout occurs, a different solution is implemented for each network link. For the local-to-remote link, the process estimator provides the estimated output value in order to generate the current (estimated) PI control action. For the remote-to-local link, a packet-based strategy is adopted. The packet includes the current PI control action and $M$ future ones to be used just in case the dropout occurs.

Summarizing, the main motivation of this paper is to introduce some control techniques (multi-rate and packet-based ones) in order to save energy (reducing the number of transmissions) while maintaining a satisfactory control performance in a NCS, where some typical problems (time-varying network-induced delays, packet dropouts, and packet disorder) must be faced.

The main contributions of this work with regard to another proposal in [20] are: 1) the remote-to-local network link is now considered, which allows not only to distribute the controller between remote and local sides, but also to deal with remote-to-local delays; 2) the network-induced delay is now stochastic. Then, probabilistic LMIs are required to ensure control system stability; 3) packet dropouts are considered in both network links. They are faced via a prediction technique which contains a state resetting procedure in order to even cope with unstable processes.

The paper is structured in the following sections. In section 3.2, the problem scenario is formally introduced. In section 3.3, control techniques used in the remote and local sides are presented. Control system stability is enunciated in terms of probabilistic LMIs in section 3.4. Simulation results in section 3.5 illustrate the benefits of the proposed control strategy. section 3.6 validates the results using a real physical process. Finally, conclusions close this contribution.

\subsection{Problem description}

The proposed NCS is depicted in Figure 3.1, where the network is placed between the remote and local sides, and can introduce time-varying delays, packet dropouts and packet disorder. The round-trip time delay for the packet sampled at the instant $k N T$ (where $k \in \mathbb{N}, T$ is the actuation period, $N T$ 


\begin{tabular}{|c|c|}
\hline \multicolumn{2}{|l|}{ Nomenclature } \\
\hline$N, t, T, N T$ & $\begin{array}{l}\text { Multiplicity, and basic, actuation } \\
\text { and sensor periods }\end{array}$ \\
\hline$\tau_{k}^{l r}, \tau_{k}^{r l}, \tau_{k}$ & $\begin{array}{l}\text { Local-to-remote, remote-to-local, } \\
\text { and round-trip time network- } \\
\text { induced delay }\end{array}$ \\
\hline$d_{k}^{l r}, d_{k}^{r l}$ & $\begin{array}{l}\text { Local-to-remote and remote-to-local } \\
\text { packet dropout occurrence }\end{array}$ \\
\hline$y_{k}^{N T}, \hat{y}_{k}^{N T}, \dot{y}_{k}^{N T}$ & $\begin{array}{l}\text { Actual, estimated, and actual or es- } \\
\text { timated, system output (at } N T)\end{array}$ \\
\hline$r_{k}^{N T}$ & Reference signal (at $N T$ ) \\
\hline$e_{P I, k}^{N T}$ & Error signal (PI input) (at $N T)$ \\
\hline$x_{k}^{N T}, \hat{x}_{k}^{N T}, \dot{x}_{k}^{N T}$ & $\begin{array}{l}\text { Actual, estimated, and actual or es- } \\
\text { timated, system state (at } N T)\end{array}$ \\
\hline$u_{P I, k}^{N T}, \hat{u}_{P I, k}^{N T}, \dot{u}_{P I, k}^{N T}$ & $\begin{array}{l}\text { Actual, estimated, and actual or es- } \\
\text { timated, PI control action (at } N T)\end{array}$ \\
\hline$\tilde{u}_{P I, k}^{T}$ & Expanded PI control action (at $T$ ) \\
\hline $\bar{u}_{P I, k}^{T}, \hat{\bar{u}}_{P I, k}^{T}, \dot{\bar{u}}_{P I, k}^{T}$ & $\begin{array}{l}\text { Actual, estimated, and actual or es- } \\
\text { timated, PI action converted at } T\end{array}$ \\
\hline$u_{P D, k}^{T, \tau_{k}}, \hat{u}_{P D, k}^{T, \tau_{k}}, \dot{u}_{P D, k}^{T, \tau_{k}}$ & $\begin{array}{l}\text { Actual, estimated, and actual or es- } \\
\text { timated, (retuned) PD control ac- } \\
\text { tion (at } T \text { ) }\end{array}$ \\
\hline$M$ & Upper bound for estimations \\
\hline$z$ & $t$-unit operator \\
\hline $\bar{z}=z^{L}, L=\frac{T}{t}$ & $T$-unit operator \\
\hline$z_{N}=\bar{z}^{N}=z^{N \cdot L}$ & $N T$-unit operator \\
\hline
\end{tabular}




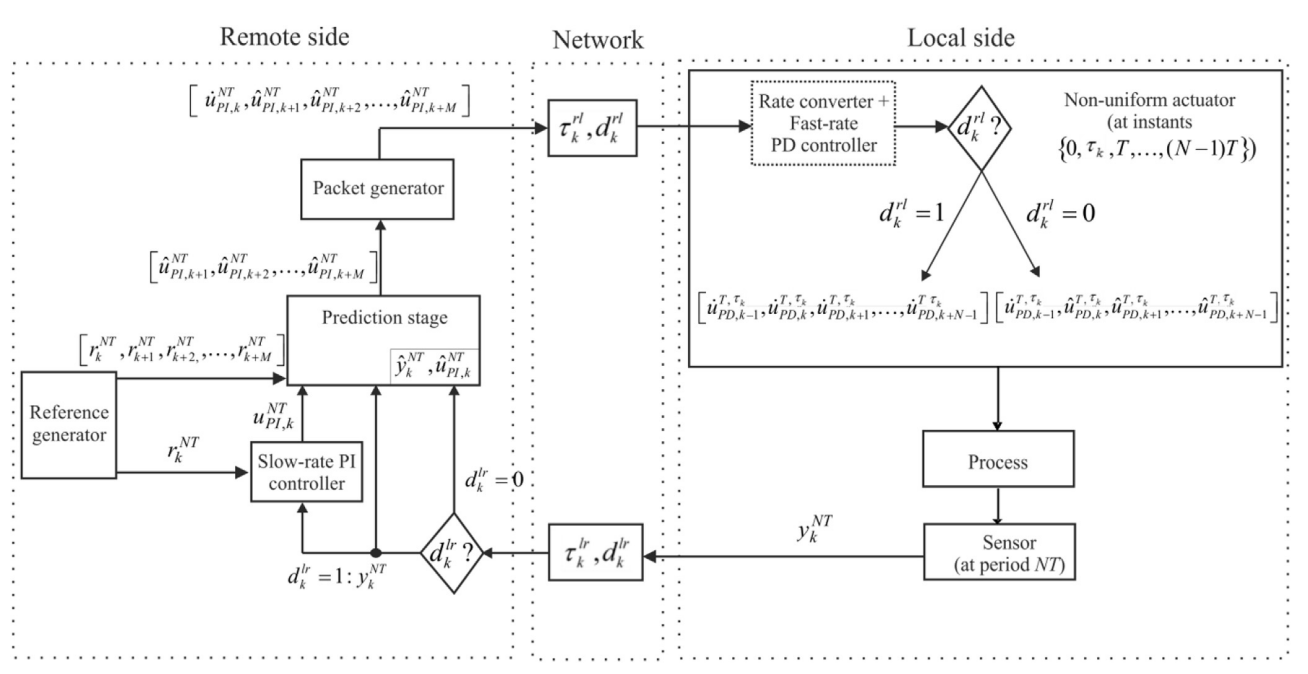

Figure 3.1: NCS scenario

is the sensor period, and $N \in \mathbb{Z}^{+}$is a parameter known as multiplicity in a dual-rate control framework [17]) is defined as

$$
\tau_{k}=\tau_{k}^{l r}+\tau_{k}^{r l}+\tau_{k}^{c}
$$

where $\tau_{k}^{c}$ can be considered as a negligible computation time delay, $\tau_{k}^{l r}$ is the local-to-remote network-induced delay, and $\tau_{k}^{r l}$, the remote-to-local one. To avoid packet disorder, $\tau_{k} \in\left[0, \tau_{\max }\right]$ must fulfill $\tau_{\max }<N T$. Since in this work, an IP network which uses UDP as the transport layer protocol is taken into account, the distribution of the round-trip time delay is a constant plus a Gamma distributed random variable, whose shape and scale parameters change with load and network segment [22]. Usually, this distribution is approximated as a generalized exponential distribution [25], whose probability density function can take this form

$$
P\left[\tau_{k}\right]= \begin{cases}\frac{1}{\phi} e^{\frac{-\left(\tau_{k}-\eta\right)}{\phi}} & , \tau_{k} \geq \eta \\ 0 & , \tau_{k}<\eta\end{cases}
$$

being the expected value of the delay $E\left[\tau_{k}\right]=\phi+\eta$ and its variance $V\left[\tau_{k}\right]=\phi^{2}$. A feasible choice of $\eta$ is the median of the delay. From $\eta$ and an experimental value of $E\left[\tau_{k}\right]$ (or the mean), $\phi$ can be easily approximated. Note that a 
common timer is supposed to be shared by the local devices in such a way that all of them are perfectly synchronized. Then, $\tau_{k}$ can be measured subtracting packet sending and receiving times, not requiring time-stamping techniques.

As well-known, when using the UDP transmission model, packet dropouts appear. This phenomenon is essentially random [21], and hence, it can be modeled as a Bernoulli distribution [1]. The variable $d_{k}^{l r}$ indicates the possible loss of the packet sent from the local side to the remote one at the instant $\mathrm{kNT}$ (similarly, $d_{k}^{r l}$ is defined for the opposite network link). In this work, both variables are considered as a Bernoulli process with probability of dropout:

$$
\begin{aligned}
& p^{l r}=\operatorname{Pr}\left[d_{k}^{l r}=0\right] \in[0,1) \\
& p^{r l}=\operatorname{Pr}\left[d_{k}^{r l}=0\right] \in[0,1)
\end{aligned}
$$

Next, the different devices included in Figure 3.1 for the NCS are presented:

- a process to be controlled: as it will be introduced in section 3.5 and section 3.6, a Cartesian robot will be used.

- a sensor, working at period $N T$, to sample the process output $y_{k}^{N T}$. Sensing at this slow rate enables to achieve energy saving by reducing network load.

- a slow-rate PI controller, which generates a PI control action $u_{P I, k}^{N T}$ from the reference $r_{k}^{N T}$ and the sample $y_{k}^{N T}$, as long as it arrives to the remote side $\left(d_{k}^{l r}=1\right)$ after $\tau_{k}^{l r}$. Otherwise $\left(d_{k}^{l r}=0\right)$, a previously estimated PI control action $\hat{u}_{P I, k}^{N T}$ will be used. Note that to detect a packet dropout in this device, a maximum waiting time $\tau_{\max }^{l r}$ is considered. If $\tau_{\text {max }}^{l r}$ expires and the packet does not arrive, it will be considered as a dropout. More information about the definition and operation mode of the slow-rate PI controller can be found in subsection 3.3.1.

- a prediction stage, which computes an array of $M$ estimated, future PI control actions $\left[\hat{u}_{P I, k+1}^{N T}, \hat{u}_{P I, k+2}^{N T}, \cdots, \hat{u}_{P I, k+M}^{N T}\right]$ from the array of the actual and future references $\left[r_{k}^{N T}, r_{k+1}^{N T}, r_{k+2}^{N T}, \cdots, r_{k+M}^{N T}\right]$, and the actual (or estimated) PI control action $u_{P I, k}^{N T}\left(\right.$ or $\hat{u}_{P I, k}^{N T}$ ), the process output $y_{k}^{N T}$ (or $\hat{y}_{k}^{N T}$ ), and the state $\hat{x}_{k}^{N T}$ (or $\hat{x}_{k}^{N T}$ ). For the sake of simplicity and brevity, both cases (actual and estimated) will be contained under the notation $\dot{u}_{P I, k}^{N T}, \dot{y}_{k}^{N T}, \dot{x}_{k}^{N T}$ on the sequel. The main goal of the prediction stage is facing packet dropouts for both network links. A more detailed scheme about this stage is depicted in Figure 3.2, where a prediction cascade 


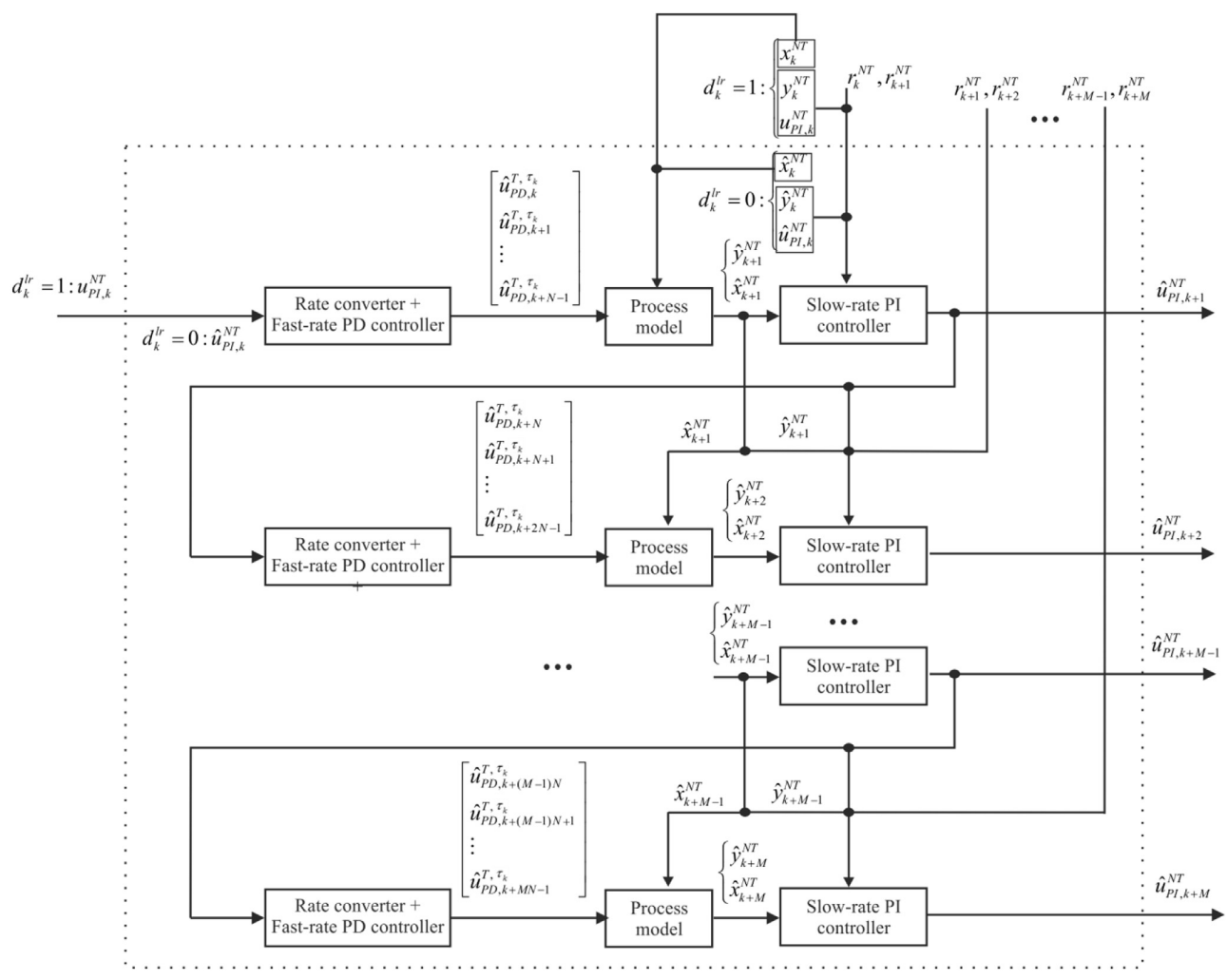

Figure 3.2: Prediction stage in detail

structure is considered. For more information about how the prediction stage works, see subsection 3.3.4.

- a packet generator, which enables to consider a packet-based control strategy by creating the packet to be sent to the local side, containing $\left[\dot{u}_{P I, k}^{N T}, \hat{u}_{P I, k+1}^{N T}, \hat{u}_{P I, k+2}^{N T}, \cdots, \hat{u}_{P I, k+M}^{N T}\right]$.

- a non-uniform actuator which includes a rate converter and a fast-rate PD controller: the rate converter converts the slow-rate PI control signal to a fast-rate one in order to be used as an input to the fast-rate PD controller (more details in subsection 3.3.2). The two main goals of the fast-rate PD controller are: 1) to achieve the required performance from a slow-rate sensing but acting at a fast rate (that is, a dual-rate control strategy [17]), 2) to compensate for the round-trip time delay, which can be measured at the local side. Depending on $d_{k}^{r l}$, two different cases can 
be considered. Figure 3.3 illustrates the case when no packet dropout occurs from remote to local sides $\left(d_{k}^{r l}=1\right)$, and Figure 3.4, the opposite case $\left(d_{k}^{r l}=0\right)$. In both cases, once the delay $\tau_{k}$ is known (or the packet dropout is detected after expiring a supposed maximum waiting time $\tau_{\text {max }}$, and hence $\tau_{k}=\tau_{\max }$ ), the controller generates the PD control signal $\left(\left[\dot{u}_{P D, k}^{T, \tau_{k}}, \dot{u}_{P D, k+1}^{T, \tau_{k}}, \cdots, \dot{u}_{P D, k+N-1}^{T, \tau_{k}}\right]\right.$ or $\left[\hat{u}_{P D, k}^{T, \tau_{k}}, \hat{u}_{P D, k+1}^{T, \tau_{k}}, \cdots, \hat{u}_{P D, k+N-1}^{T, \tau_{k}}\right]$, depending on $d_{k}^{r l}$ ) by adopting a gain scheduling approach [20]. The control signal is applied to the process by the actuator in a non-uniform way, since the last control action of the previous sensor period $N T, \dot{u}_{P D, k-1}^{T, \tau_{k}}$, is held while no new one can be applied due to the delay $\tau_{k}$. Note, $\tau_{\max }$ could fulfill $\tau_{\max } \geq d T, d \in \mathbb{Z}^{+}$, then the first $d$ control actions would not be applied, and hence, the control performance could worsen. More information about the fast-rate PD controller can be found in subsection 3.3.3.

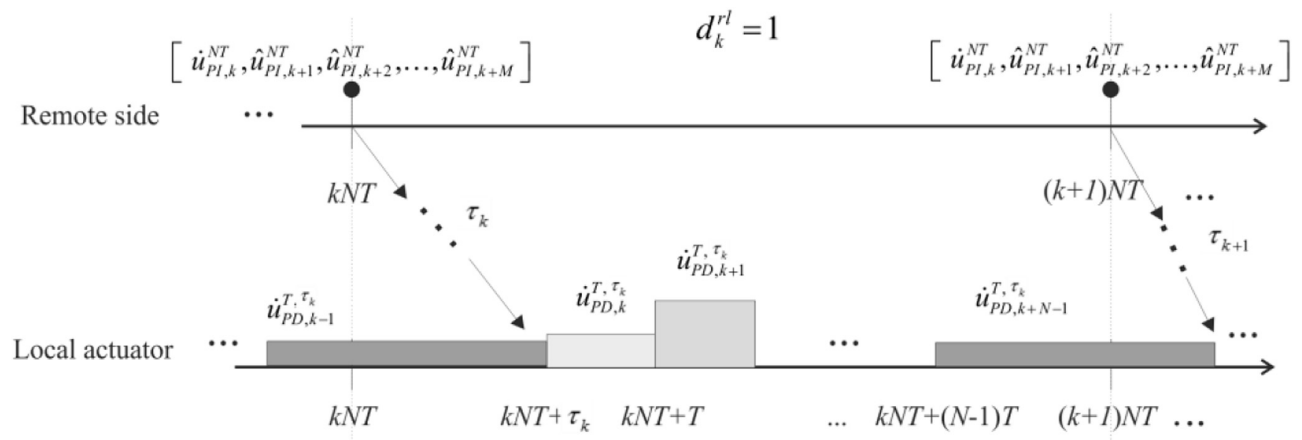

Figure 3.3: No packet dropout $\left(d_{k}^{r l}=1\right)$

\subsection{Packet-based control strategy. Prediction stage}

In this section, the packet-based control strategy proposed in this work is formulated in subsections 3.3.1 (slow-rate PI controller), 3.3.2 (rate converter), and 3.3.3 (fast-rate PD controller). Since packet dropouts could occur in both network links, each control stage must consider two cases: a) no packet dropout, b) packet dropout. At the end of the section (in subsection 3.3.4), the prediction stage is enunciated.

First of all, let us define the transfer function of the continuous plant to be controlled as $G_{p}(s)$. By using the Z-transform at different periods plus a 


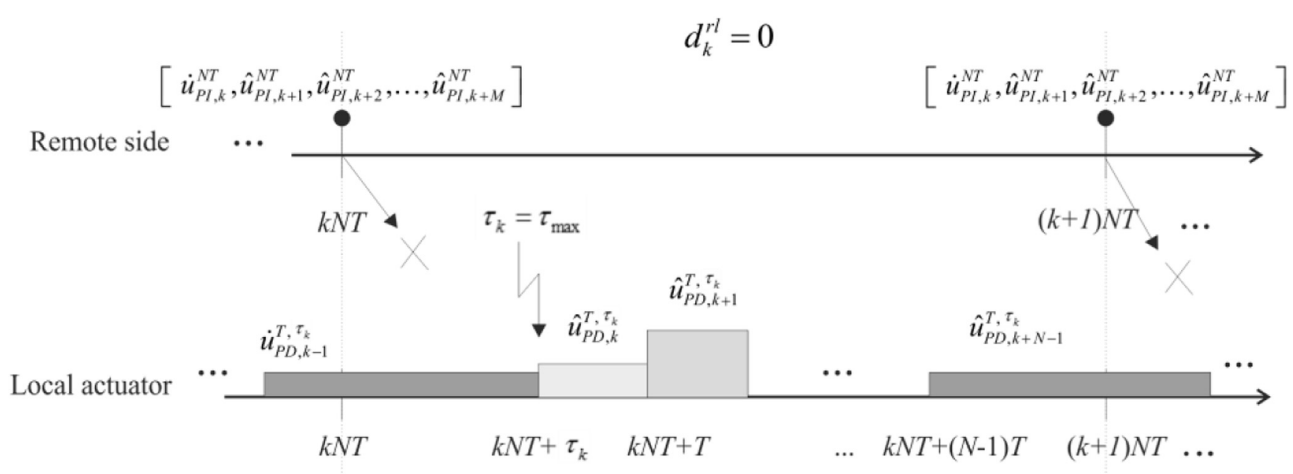

Figure 3.4: Packet dropout $\left(d_{k}^{r l}=0\right)$

zero order hold device $H(s)$, different discrete-time versions for $G_{p}(s)$ can be calculated. Therefore, denoting $\bar{z}=z^{L}$ and $z_{N}=\bar{z}^{N}=z^{N \cdot L}$ :

$$
\begin{aligned}
& G^{N T}\left(z_{N}\right) \triangleq Z_{N T}\left[H_{N T} G_{p}(s)\right]=\frac{Y^{N T}\left(z_{N}\right)}{U^{N T}\left(z_{N}\right)} ; \\
& G^{T}(\bar{z}) \triangleq Z_{T}\left[H_{T} G_{p}(s)\right]=\frac{Y^{T}(\bar{z})}{U^{T}(\bar{z})} ; \\
& G^{t}(z) \triangleq Z_{t}\left[H_{t} G_{p}(s)\right]=\frac{Y^{t}(\bar{z})}{U^{t}(\bar{z})}, \quad t<T: \quad t \cdot L=T, \quad L \in \mathbb{Z}^{+}
\end{aligned}
$$

In addition, the consequent state-space representations for each case can be enunciated as

$$
\begin{aligned}
& \left\{\begin{array}{l}
x_{k+1}^{N T}=A^{N T} x_{k}^{N T}+B^{N T} u_{k}^{N T} \\
y_{k}^{N T}=C^{N T} x_{k}^{N T}
\end{array}\right. \\
& \begin{cases}x_{k+1}^{T} & =A^{T} x_{k}^{T}+B^{T} u_{k}^{T} \\
y_{k}^{T} & =C^{T} x_{k}^{T}\end{cases} \\
& \begin{cases}x_{k+1}^{t} & =A^{t} x_{k}^{t}+B^{t} u_{k}^{t} \\
y_{k}^{t} & =C^{t} x_{k}^{t}\end{cases}
\end{aligned}
$$

Secondly, let us consider a continuous PID controller which is designed according to classical methods in order to achieve certain specifications for the process 
to be controlled. This is the configuration considered for the continuous PID controller:

$$
G_{P I D}(s)=K_{p}\left(1+T_{d} s+\frac{1}{T_{i} s}\right)
$$

\subsubsection{Slow-rate PI controller}

The following two cases must be considered:

a) No dropout $\left(d_{k}^{l r}=1\right)$ : The PI controller working at period NT is enunciated as

$$
G_{P I}^{N T}\left(z_{N}\right)=K_{P I} \frac{z_{N}-\left(1-\frac{N T}{T_{i}}\right)}{z_{N}-1}=\frac{U_{P I}^{N T}\left(z_{N}\right)}{E_{P I}^{N T}\left(z_{N}\right)}
$$

being $U_{P I}^{N T}\left(z_{N}\right)$ the PI control signal, $E_{P I}^{N T}\left(z_{N}\right)$ the error signal, and $K_{P I}, T_{i}$ the gains of the PI controller (usually, $K_{P I}=1$ ). The PI control signal is obtained as

$$
U_{P I}^{N T}\left(z_{N}\right)=G_{P I}^{N T}\left(z_{N}\right) E_{P I}^{N T}\left(z_{N}\right)=G_{P I}^{N T}\left(z_{N}\right)\left(R_{P I}^{N T}\left(z_{N}\right) Y_{P I}^{N T}\left(z_{N}\right)\right)
$$

and, from (3.7), the difference equation for the PI controller (with $K_{P I}=$ 1) will be

$$
\begin{aligned}
u_{P I, k}^{N T} & =u_{P I, k-1}^{N T}+e_{P I, k}^{N T}-\left(1-\frac{N T}{T_{i}}\right) e_{P I, k-1}^{N T} \\
& =u_{P I, k-1}^{N T}+\left(r_{k}^{N T}-y_{k}^{N T}\right)-\left(1-\frac{N T}{T_{i}}\right)\left(r_{k-1}^{N T}-y_{k-1}^{N T}\right)
\end{aligned}
$$

b) Dropout $\left(d_{k}^{l r}=0\right)$ : In this case, instead of using the actual PI control signal in (3.8), the estimated one $\hat{U}_{P I}^{N T}\left(z_{N}\right)$ must be used. This signal is previously generated at the prediction stage according to subsection 3.3.4. 


\subsubsection{Rate converter}

As it is well-known [17], a rate converter $\left[H^{N T}\right]^{T}(\bar{z})$ between slow (remote) and fast (local) controllers is required. Its goal is to convert the slow rate PI control signal to a fast rate one in order to be used as an input to the fast rate PD controller. This operation can be carried out either at the remote side (sending the converted signal to the local side) or directly at the local side (this is the option used in this work). Two cases are considered:

a) No dropout $\left(d_{k}^{l r}=1\right)$ : The rate converter considers the actual slow-rate PI control signal $U_{P I}^{N T}\left(z_{N}\right)$ to obtain the held fast-rate one $\bar{U}_{P I}^{T}(\bar{z})$. As, in this work, step references are considered, the rate converter becomes a digital zero order hold:

$$
\begin{array}{r}
{\left[H^{N T}\right]^{T}(\bar{z})=\frac{\bar{U}_{P I}^{T}(\bar{z})}{\left[U_{P I}^{N T}\left(z_{N}\right)\right]^{T}}=\frac{1-\bar{z}^{-N}}{1-\bar{z}^{-1}} \rightarrow} \\
\bar{U}_{P I}^{T}(\bar{z})=\left[H^{N T}\right]^{T}(\bar{z})\left[U_{P I}^{N T}\left(z_{N}\right)\right]^{T}
\end{array}
$$

Note that $U_{P I}^{N T}\left(z_{N}\right)$ is required to be used in an expanded way $\left[U_{P I}^{N T}\left(z_{N}\right)\right]^{T}$, that is,

$$
\begin{aligned}
{\left[U_{P I}^{N T}\right]^{T}\left(z_{N}\right) } & \triangleq \tilde{U}_{P I}^{T}(\bar{z}) \\
& \triangleq \sum_{k=0}^{\infty} \tilde{u}_{P I, k}^{T} \bar{z}^{-k}:\left\{\begin{array}{ll}
\tilde{u}_{P I, k}^{T}=u_{P I, k}^{T}, & \forall k=\lambda N \\
\tilde{u}_{P I, k}^{T}=0, & \forall k \neq \lambda N
\end{array}, \lambda \in \mathbb{Z}^{+}\right.
\end{aligned}
$$

More information can be found in [17]

b) Dropout $\left(d_{k}^{l r}=0\right)$ : Now, the rate converter considers the estimated PI control signal $\hat{U}_{P I}^{N T}\left(z_{N}\right)$, that is

$$
\hat{\bar{U}}_{P I}^{T}(\bar{z})=\left[H^{N T}\right]^{T}(\bar{z})\left[\hat{U}_{P I}^{N T}\left(z_{N}\right)\right]^{T}
$$

As it was said in section 3.2, for the sake of simplicity and brevity, both cases $((3.10)$ and $(3.12))$ will be contained under the notation $\dot{\bar{U}}_{P I}^{T}(\bar{z})$ from now on. 


\subsubsection{Fast-rate PD controller}

a) No dropout $\left(d_{k}^{l r}=1\right)$ : After (3.1), the round-trip time delay was defined to fulfill $\tau_{k} \in\left[0, \tau_{\max }\right]$. Let us suppose $\tau_{\max }<T$ (for example, $\left.\tau_{\max }=T-t\right)$ in order to inject $N$ control actions for each sensor period $N T$ (remember Figure 3.3). The PD controller, working at period $T$, is enunciated as

$$
\begin{array}{r}
G_{P D}^{T, \tau_{k}}(\bar{z})=K_{P D}^{\tau_{k}} \frac{\bar{z}\left(1+\frac{T_{d}^{\tau_{k}}}{T}\right)-\frac{T_{d}^{\tau_{k}}}{T}}{\bar{z}}=\frac{\dot{U}_{P D}^{T, \tau_{k}}(\bar{z})}{\dot{\bar{U}}_{P I}^{T}(\bar{z})} \rightarrow \\
\dot{U}_{P D}^{T, \tau_{k}}(\bar{z})=G_{P D}^{T, \tau_{k}}(\bar{z}) \dot{\bar{U}}_{P I}^{T}(\bar{z})
\end{array}
$$

where $K_{P D}^{\tau_{k}}, T_{d}^{\tau_{k}}$ are the gains of the PD controller, which are retuned according to $\tau_{k}$ via the gain-scheduling algorithm presented in [20]. Note that the notation $\dot{U}_{P D}^{T, \tau_{k}}(\bar{z})$ represents the PD control signal obtained either from the actual PI control signal $\bar{U}_{P I}^{T}(\bar{z})$ (in (3.10)) or from its estimation $\hat{\bar{U}}_{P I}^{T}(\bar{z})$ (in (3.12)). At the current sensor period, in addition to the last PD control action of the previous period (which remains held), new $N$ PD control actions are applied to the plant after $\tau_{k}$ (remember Figure 3.3). These $N$ actions are obtained after iterating the difference equation deduced from (3.13) $N$ times, that is

$$
\dot{u}_{P D, k}^{T, \tau_{k}}=K_{P D}^{\tau_{k}}\left(1+\frac{T_{d}^{\tau_{k}}}{T}\right) \dot{\bar{u}}_{P I, k}^{T}-K_{P D}^{\tau_{k}}\left(\frac{T_{d}^{\tau_{k}}}{T}\right) \dot{\bar{u}}_{P I, k-1}^{T}
$$

As commented in section 3.2, due to the delay, these actions will be applied following a non-uniform pattern. Then, a basic period $t$ is required to adapt the non-uniformity to the delay in such a way that the actuation pattern inside the sensor period $N T$ will take this form (where $l=0 . . L N-1)$ :

$$
\begin{cases}\dot{u}_{P D, \tau_{k}}^{T, \tau_{k}}, & l t=0 . . \tau_{k} \\ \dot{u}_{P D, \tau_{k},}^{T, \tau_{k}}, & l t=\tau_{k} . . T \\ \dot{u}_{P D, k+1}^{T, \tau_{k}}, & l t=T . .2 T \\ \vdots & \\ \dot{u}_{P D, k+N-1}^{T, \tau_{k}}, & l t=(N-1) T . . N T\end{cases}
$$

b) Dropout $\left(d_{k}^{l r}=0\right)$ : The estimated PI control signal $\hat{\bar{U}}_{P I}^{T}(\bar{z})$ (to be defined in the last step in subsection 3.3.4) is now required. This control signal 
is available at the local side, since it was calculated at the remote side in a previous iteration and sent to the local side in a previous successful communication.

The time $\tau_{\max }=T-t$ is considered as the maximum waiting time established to detect a packet dropout (remember Figure 3.4). In this way, $N$ PD control actions (in addition to the held action from the previous sensor period) are guaranteed to be applied in the current sensor period (as in (3.15)). In this case, with certainty, the PD control signal is an estimated one, since it depends on the estimated PI control signal. Then, with $\tau_{k}=\tau_{\max }$

$$
\hat{U}_{P D}^{T, \tau_{k}}(\bar{z})=G_{P D}^{T, \tau_{k}}(\bar{z}) \hat{\bar{U}}_{P I}^{T}(\bar{z})
$$

From (3.14), but considering estimated signals, the set of $N$ control actions can be computed and applied according to the next non-uniform actuation pattern inside the sensor period $N T$ (where $t$ is the basic period, and $l=0 . . L N-1)$ :

$$
\begin{cases}\dot{u}_{P D, k-1}^{T, \tau_{k}}, & l t=0 . . \tau_{k} \\ \hat{u}_{P D, \tau_{k}}^{T, k}, & l t=\tau_{k} . . T \\ \hat{u}_{P D, k+1}^{T, \tau_{k}}, & l t=T . .2 T \\ \vdots & \\ \hat{u}_{P D, k+N-1}^{T, \tau_{k}}, & l t=(N-1) T . . N T\end{cases}
$$

\subsubsection{Prediction Stage}

The prediction algorithm is executed $M$ times ( $M$ is the upper bound of consecutive packet dropouts) following a cascade structure (remember Figure 3.2) in order to generate the packet which includes the future, estimated $M$ PI control actions $\left[\hat{u}_{P I, k+1}^{N T}, \hat{u}_{P I, k+2}^{N T}, \cdots, \hat{u}_{P I, k+M}^{N T}\right]$. This packet is computed for every sensor period at the remote side, and it is sent to the local side in order to be stored, and used if subsequent dropouts occur through the remote-to-local communication. Considering a for-loop where $i=1 . . M$, the statements of the prediction algorithm included in the loop are based on the next steps:

1. Resetting of the initial state: If the current state sensed at period $N T$, $x_{k}^{N T}$, is available at the remote side (that is, no dropout occurs when being sent via the local-to-remote network link), a resetting of the initial condition for the state at period $t$ and $T$ can be carried out. This operation can be executed when $i=1$, and it is required in order to deal with 
unstable plants or marginally stable plants $[23,24]$ such as the one used in this work. For the rest of iterations of the algorithm $(i=2 . . M)$, or if the current state was dropped (for $i=1$ ), the updating is computed from the estimated state $\hat{x}_{k+i-1}^{N T}$ (to be defined in step 3). As in section 3.2, to contemplate every situation, let us define a generic (actual or estimated) state $\dot{x}_{k}^{N T}$. Therefore, the resetting carried out in each iteration is

$$
\begin{cases}i=1: & \hat{x}_{k}^{T} \leftarrow \dot{x}_{k}^{N T} ; \quad \hat{x}_{k}^{t} \leftarrow \dot{x}_{k}^{N T} \\ i>1: & \hat{x}_{k+(i-1) N}^{T} \leftarrow \hat{x}_{k+i-1}^{N T} ; \quad \hat{x}_{k+(i-1) L N}^{t} \leftarrow \hat{x}_{k+i-1}^{N T}\end{cases}
$$

2. Estimation of the $N \mathrm{PD}$ control actions either from the estimated PI control signal $\hat{U}_{P I}^{T}(\bar{z})$ (it can occur for $i \geq 1$ ) or from the actual one $\bar{U}_{P I}^{T}(\bar{z})$ (it can only occur for $i=1$ ). Both cases assume the previous rate conversion ((3.12) or (3.10), respectively).

Similarly to (3.14), the estimated control signal is computed by iterating the next difference equation for $j=0 . . N-1$. Each iteration $i$ for the prediction algorithm is calculated as follows

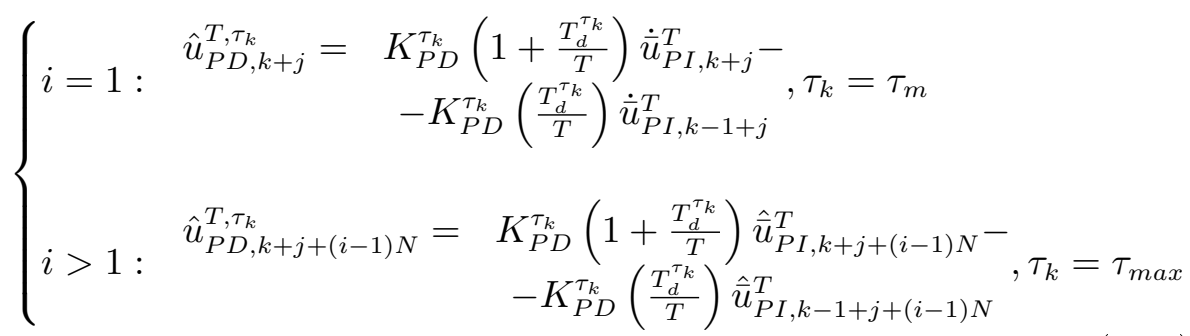

where now, the equation for the first iteration of the prediction algorithm $(i=1)$ is calculated supposing a successful remote-to-local communication (the packet which includes the estimated PI control actions will arrive to the local side) but unknowing the consequent remote-to-local delay (this information is not available at this moment) and hence, the round-trip time delay. This is the reason of adopting the statistical mode of the delay distribution $\tau_{m}$ as the delay to be considered at the first iteration. The mode can be obtained via a previous statistical analysis about the delay nature. For the rest of iterations (when $i>1$ ), the considered delay to generate the $\mathrm{PD}$ control actions is the maximum waiting time established to detect a dropout, that is, $\tau_{k}=\tau_{\max }$, since the next $M-1$ packets are assumed to be dropped.

3. Estimation of the next state and output at period NT from the estimated PD control actions. As in (3.15) and (3.16), the basic period $t$ is used. 
In this case, for each iteration of the prediction algorithm $i=1 . . M$, the next state-space representation is calculated by iterating for $l=0 . . L N-1$

$$
\left\{\begin{array}{l}
\hat{x}_{k+1+l+(i-1) L N}^{t}=A^{t} \hat{x}_{k+l+(i-1) L N}^{t}+B^{t}\left\langle\hat{u}_{P D, k+l+(i-1) L N}^{T, \tau_{k}}\right\rangle^{t} \\
\hat{y}_{k+1+l+(i-1) L N}^{t}=C^{t} \hat{x}_{k+1+l+(i-1) L N}^{t}
\end{array}\right.
$$

where, for simplicity and brevity, let us represent by means of $\left\langle\hat{u}_{P D, k+l+(i-1) L N}^{T, \tau_{k}}\right\rangle^{t}$ the sequence of the $N+1 \mathrm{PD}$ control actions included in each sensor period by holding them at period $t$, that is, applying them via a non-uniform actuation pattern such as the one used in (3.17). As a result of iterating (3.20) for all $i$, the $M$ estimated states and outputs at period $N T$, $\hat{x}_{k+i}^{N T}, \hat{y}_{k+i}^{N T}$, are obtained.

4. Estimation of the PI control signal $\hat{U}_{P I}^{N T}\left(z_{N}\right)$ from the estimated output signal $\hat{Y}^{N T}\left(z_{N}\right)$. Note that, particularly for the first iteration of the prediction algorithm $(i=1)$, the actual output $y_{k}^{N T}$ can be used if it is available at the remote side, that is, if no dropout occurs when being sent through the local-to-remote network link $\left(d_{k}^{l r}=1\right)$. Then, the actual PI control action $u_{P I, k}^{N T}$, which is generated by the output $y_{k}^{N T}$ (remember (3.9)), can also be used. In this way, similarly to step 1, a resetting of the initial condition for the PI controller $\left(u_{P I, k}^{N T}\right)$ is carried out in order to compute the next estimated PI control action $\hat{u}_{P I, k+1}^{N T}$. This operation is useful due to the marginally stable open-loop nature of the PI controller [23, 24]. As usual, in order to contemplate every situation in the prediction algorithm, let us define a generic (actual or estimated) output $\dot{y}_{k}^{N T}$, and a generic (actual or estimated) control action $\dot{u}_{P I, k}^{N T}$. Therefore, similarly to (3.9), the iterations $i$ of the prediction algorithm take the form

$$
\begin{cases}i=1: & \hat{u}_{P I, k+1}^{N T}=\dot{u}_{P I, k}^{N T}+\left(r_{k+1}^{N T}-\hat{y}_{k+1}^{N T}\right)-\left(1-\frac{N T}{T_{i}}\right)\left(r_{k}^{N T}-\dot{y}_{k}^{N T}\right) \\ i>1: & \hat{u}_{P I, k+i}^{N T}=\hat{u}_{P I, k+i-1}^{N T}+\left(r_{k+i}^{N T}-\hat{y}_{k+i}^{N T}\right)-\left(1-\frac{N T}{T_{i}}\right)\left(r_{k+i-1}^{N T}-\hat{y}_{k+i-1}^{N T}\right)\end{cases}
$$




\subsection{Stability analysis}

\subsubsection{Closed-loop model via lifting}

Let us consider a continuous linear time-invariant plant, which admits a statespace realization $\Sigma=(\bar{A}, \bar{B}, C, D)$, with suitable dimensions. Being $\xi$ an arbitrary real number, one can denote $B(\xi)=\int_{0}^{\xi} e^{\bar{A} \gamma} \bar{B} d \gamma$ if $\xi>0$, or $B(\xi)=0$ if $\xi \leq 0$. The discrete time sampled-data model at period $T$ of the previous plant was presented in (3.5), being $A^{T}=e^{\bar{A} T} ; \quad B^{T}=B(T) ; \quad C^{T}=C$.

In order to reflect the dual-rate sampling with non-uniform actuation (inputs are applied at instants $\lambda_{i}(i=0,1, \cdots, N)$; concretely, as detailed in section 3.2, the actuation pattern inside a sensor period is $\left\{0, \tau_{k}, T, 2 T, \ldots\right.$ , $(N-1) N\}$, and hence $\left.\lambda_{0}=0 ; \lambda_{1}=\tau_{k} ; \lambda_{2}=T ; \cdots ; \lambda_{N}=(N-1) T\right)$, the control system can be modeled via state-space representation adopting the so-called lifting methodology [26]. Then, let us represent the process as:

$$
\begin{aligned}
x_{k+1}^{N T} & =A_{p} x_{k}^{N T}+B_{p} U_{P D}^{T, \tau_{k}} \\
y_{k}^{N T} & =C_{p} x_{k}^{N T}
\end{aligned}
$$

where

- $A_{p}=\left(\begin{array}{cc}e^{\bar{A} N T} & B_{0}^{*} \\ 0 & 0\end{array}\right) ; \quad B_{p}=\left(\begin{array}{ccc}B_{1}^{*} & \cdots & B_{N}^{*} \\ 0 & \cdots & 1\end{array}\right) \quad C_{p}=\left(\begin{array}{ll}C & 0\end{array}\right) ;$ being $B_{i}^{*}=B\left(\lambda_{i+1}-\lambda_{i}\right) e^{\bar{A}\left(N T-\lambda_{i+1}\right)}$.

- $U_{P D}^{T, \tau_{k}}\left[\begin{array}{llll}u_{P D, k}^{T, \tau_{k}} & u_{P D, k+1}^{T, \tau_{k}} & \cdots & u_{P D, k+N+1}^{T, \tau_{k}}\end{array}\right]^{t r}$ is a generic array of $N$ control signals $\left((\cdot)^{t r}\right.$ means transpose function) to indistinctly represent the $N$ computed actions that appear both in (3.15) and in (3.17), that is, all the actions included in these equations except the last action from the previous sensing period, $U_{P D, k-1}^{T, \tau_{k}}$. This action $U_{P D, k-1}^{T, \tau_{k}}$ is included in the state $x_{k}^{N T}$ as a state variable which is memorised from one sensing period to the next one, such as in [18], equation (13).

The predictor stage can be modelled as follows:

$$
\hat{x}_{k+1}^{N T} \begin{cases}A_{p} \hat{x}_{k}^{N T}+B_{p} U_{P D}^{T, \tau_{k}}, & \text { if } d_{k}^{l r}=0 \\ A_{p} x_{k}^{N T}+B_{p} U_{P D}^{T, \tau_{k}}, & \text { if } d_{k}^{l r}=1\end{cases}
$$


For the sake of simplicity and brevity, let us consider the following assumptions to define the state-space representation of the delay-dependent controller:

1. if setpoints are constant, we can assume them to be zero without loss of generality, and hence $e_{k}^{N T}=-y_{k}^{N T}$

2. the control system behaviour when $d_{k}^{l r}=0$ is similar to the one when $d_{k}^{r l}=0$ from the point of view of computing the control signal $U_{P D}^{T, \tau_{k}}$ from the estimated output $\hat{y}_{k}^{N T}$. That is, when $d_{k}^{r l}=0$, the control signal $U_{P D}^{T, \tau_{k}}$ to be applied at iteration $k$ is generated from a PI control action which was previously computed at the predictor stage taking into account an output estimation for the time instant $k, \hat{y}_{k}^{N T}$. When $d_{k}^{l r}=0$, obviously the controller needs $\hat{y}_{k}^{N T}$ to calculate $U_{P D}^{T, \tau_{k}}$.

3. the dual-rate controller can be defined as a cascade-connected system.

After manipulating the difference equations (3.9) and (3.14), and assuming such as in [18] (Section 5) that the proportional and integral actions are generated at the slow period, and the derivative one (which has relationship with anticipation and high-frequency behavior) is concentrated in the first sample, the dual-rate controller can take this lifted expression

$$
\begin{aligned}
& \left\{\begin{array}{l}
\varphi_{k+1}^{N T}=A_{c} \varphi_{k}^{N T}-B_{c} \hat{y}_{k+1}^{N T}+\bar{B}_{c} \hat{y}_{k}^{N T} \\
U_{P D}^{T, \tau_{k}}=C_{c}\left(\tau_{k}\right) \varphi_{k}^{N T}
\end{array}, \quad \text { if } d_{k}^{l r}=0 \text { or } d_{k}^{r l}=0\right. \\
& \left\{\begin{array}{l}
\varphi_{k+1}^{N T}=A_{c} \varphi_{k}^{N T}-B_{c} y_{k+1}^{N T}+\bar{B}_{c} y_{k}^{N T} \\
U_{P D}^{T, \tau_{k}}=C_{c}\left(\tau_{k}\right) \varphi_{k}^{N T}
\end{array}, \text { if } d_{k}^{l r}=d_{k}^{r l}=1\right.
\end{aligned}
$$

where $\varphi_{k}^{N T}=\left(\begin{array}{ll}U_{P I, k}^{N T} & \mu_{k}^{N T}\end{array}\right)^{t r}$, being $\mu_{k}^{N T}=U_{P I, k-1}^{N T}$, and

$$
\begin{gathered}
A_{c}=\left(\begin{array}{ll}
1 & 0 \\
1 & 0
\end{array}\right) ; \quad B_{c}=\left(\begin{array}{c}
1 \\
0
\end{array}\right) ; \quad \bar{B}_{c}=\left(\begin{array}{c}
\left.1-\frac{N T}{T_{i}}\right) \\
0
\end{array}\right) ; \\
C_{c}\left(\tau_{k}\right)=\left(\begin{array}{cc}
K_{P D}^{\tau_{k}}\left(1+\frac{T_{d}^{\tau_{k}}}{T}\right) & -K_{P D}^{\tau_{k}} \frac{T_{d}^{\tau_{k}}}{T} \\
K_{P D}^{\tau_{k}} & 0 \\
\vdots & \vdots \\
K_{P D}^{\tau_{k}} & 0
\end{array}\right)
\end{gathered}
$$

To obtain the closed-loop model, the following definition is used 


$$
v_{k}^{N T}=y_{k}^{N T}-\hat{y}_{k}^{N T}=C_{p}\left(x_{k}^{N T}-\hat{x}_{k}^{N T}\right)
$$

and this state variable is adopted $\bar{x}_{k}^{N T}=\left(\begin{array}{lll}x_{k}^{N T} & \varphi_{k}^{N T} & v_{k}^{N T}\end{array}\right)^{t r}$. Some manipulations lead to the closed-loop model:

$$
\bar{x}_{k+1}^{N T}= \begin{cases}A_{c l, 0}\left(\tau_{k}\right) \bar{x}_{k}^{N T} & \text { if } d_{k}^{l r}=0 \text { or } d_{k}^{r l}=0 \\ A_{c l, 1}\left(\tau_{k}\right) \bar{x}_{k}^{N T} & \text { if } d_{k}^{l r}=d_{k}^{r l}=1\end{cases}
$$

where

$$
\begin{aligned}
A_{c l, 0}\left(\tau_{k}\right) & =\left[\begin{array}{ccc}
A_{p} & B_{p} C_{c}\left(\tau_{k}\right) & 0 \\
\bar{B}_{c} C_{p}-B_{c} C_{p} A_{p} & A_{c}-B_{c} C_{p} B_{p} C_{c}\left(\tau_{k}\right) & -\bar{B}_{c}-A_{p} B_{c} \\
0 & 0 & A_{p}
\end{array}\right] \\
A_{c l, 1}\left(\tau_{k}\right) & =\left[\begin{array}{ccc}
A_{p} & B_{p} C_{c}\left(\tau_{k}\right) & 0 \\
\bar{B}_{c} C_{p}-B_{c} C_{p} A_{p} & A_{c}-B_{c} C_{p} B_{p} C_{c}\left(\tau_{k}\right) & 0 \\
0 & 0 & 0
\end{array}\right]
\end{aligned}
$$

\subsubsection{Closed-loop stability}

Similarly to $[9,19]$, the next Theorem can be enunciated to assess the closedloop system stability.

Theorem: Given $P\left[\tau_{k}\right]$ in (3.2), the closed-loop system in (3.27) is stable if there exists a positive definitive solution $Q=Q^{t r}>0$ for the following probabilistic LMIs

$$
\begin{aligned}
& \sum_{j=1}^{l} P\left[\vartheta_{j}\right] A_{c l, 0}^{t r}\left(\vartheta_{j}\right) \cdot Q \cdot A_{c l, 0}\left(\vartheta_{j}\right)-Q<0 \\
& \sum_{j=1}^{l} P\left[\vartheta_{j}\right] A_{c l, 1}^{t r}\left(\vartheta_{j}\right) \cdot Q \cdot A_{c l, 1}\left(\vartheta_{j}\right)-Q<0
\end{aligned}
$$

where $\vartheta$ is a dummy parameter ranging in a set $\Theta$ where $\tau_{k}$ is assumed to take values in, being $\Theta$ an interval $\left[0, \tau_{\max }\right]$, and $l$ the number of equally spaced values to get a dense enough gridding in $\vartheta$. (3.29) can be solved by means of widely known methods [27]. 
Proof: Let $V_{k}^{N T}=\left(\bar{x}_{k}^{N T}\right)^{t r} \cdot Q \cdot \bar{x}_{k}^{N T}$ be a Lyapunov candidate. Taking $E\left[V_{k}^{N T}\right]$ as the statistical expectation for the Lyapunov function, and assuming a probabilistic LMI gridding procedure, then the expectation of the increment $E\left[\Delta V_{k}^{N T}\right]$ along subsystem $A_{c l, 0}\left(\tau_{k}\right)$ in (3.27) can be obtained as follows

$$
\begin{aligned}
& E\left[\Delta V_{k}^{N T}\right]=E\left[V_{k+1}^{N T}-V_{k}^{N T}\right]=E\left[\left(\bar{x}_{k+1}^{N T}\right)^{t r} \cdot Q \cdot \bar{x}_{k+1}^{N T}-\left(\bar{x}_{k}^{N T}\right)^{t r} \cdot Q \cdot \bar{x}_{k}^{N T}\right]= \\
& =E\left[\left(A_{c l, 0}\left(\tau_{k}\right) \cdot \bar{x}_{k}^{N T}\right)^{t r} \cdot Q \cdot A_{c l, 0}\left(\tau_{k}\right) \cdot \bar{x}_{k}^{N T}-\left(\bar{x}_{k}^{N T}\right)^{t r} \cdot Q \cdot \bar{x}_{k}^{N T}\right]= \\
& =E\left[\left(\bar{x}_{k}^{N T}\right)^{t r} A_{c l, 0}^{t r}\left(\tau_{k}\right) \cdot Q \cdot A_{c l, 0}\left(\tau_{k}\right) \cdot \bar{x}_{k}^{N T}-\left(\bar{x}_{k}^{N T}\right)^{t r} \cdot Q \cdot \bar{x}_{k}^{N T}\right]= \\
& =E\left[\left(\bar{x}_{k}^{N T}\right)^{t r}\left(A_{c l, 0}^{t r}\left(\tau_{k}\right) \cdot Q \cdot A_{c l, 0}\left(\tau_{k}\right)-Q\right) \cdot \bar{x}_{k}^{N T}\right]= \\
& =\left(\bar{x}_{k}^{N T}\right)^{t r}\left(\sum_{j=1}^{l} P\left[\vartheta_{j}\right] A_{c l, 0}^{t r}\left(\vartheta_{j}\right) \cdot Q \cdot A_{c l, 0}\left(\vartheta_{j}\right)-Q\right) \bar{x}_{k}^{N T}<0
\end{aligned}
$$

A similar development for subsystem $A_{c l, 1}\left(\tau_{k}\right)$ leads to (3.29).

\subsection{Simulation results}

\subsubsection{Simulation data}

In this section, a particular case for the proposed NCS is presented. The control solution is shown by simulation, which is based on a model of a plant available in the laboratory in order to then validate the results experimentally (in section 3.6).

The process selected to be controlled is a Cartesian robot manufactured by Inteco, specifically, the 3D CRANE module (see in Figure 3.5). The rail measures of this plant for each axis are: $\mathrm{X}=0.050 \mathrm{~m}, \mathrm{Y}=0.040 \mathrm{~m}, \mathrm{Z}=0.050 \mathrm{~m}$.

Focusing on the $\mathrm{X}$ axis, it is identified, obtaining the next model

$$
G_{p}(s)=\frac{6.3}{s(s+17.7)} m / c . a . u .
$$

where c.a.u. means control action units, which are generated by a PWM signal normalized in the range $[0,1]$. 


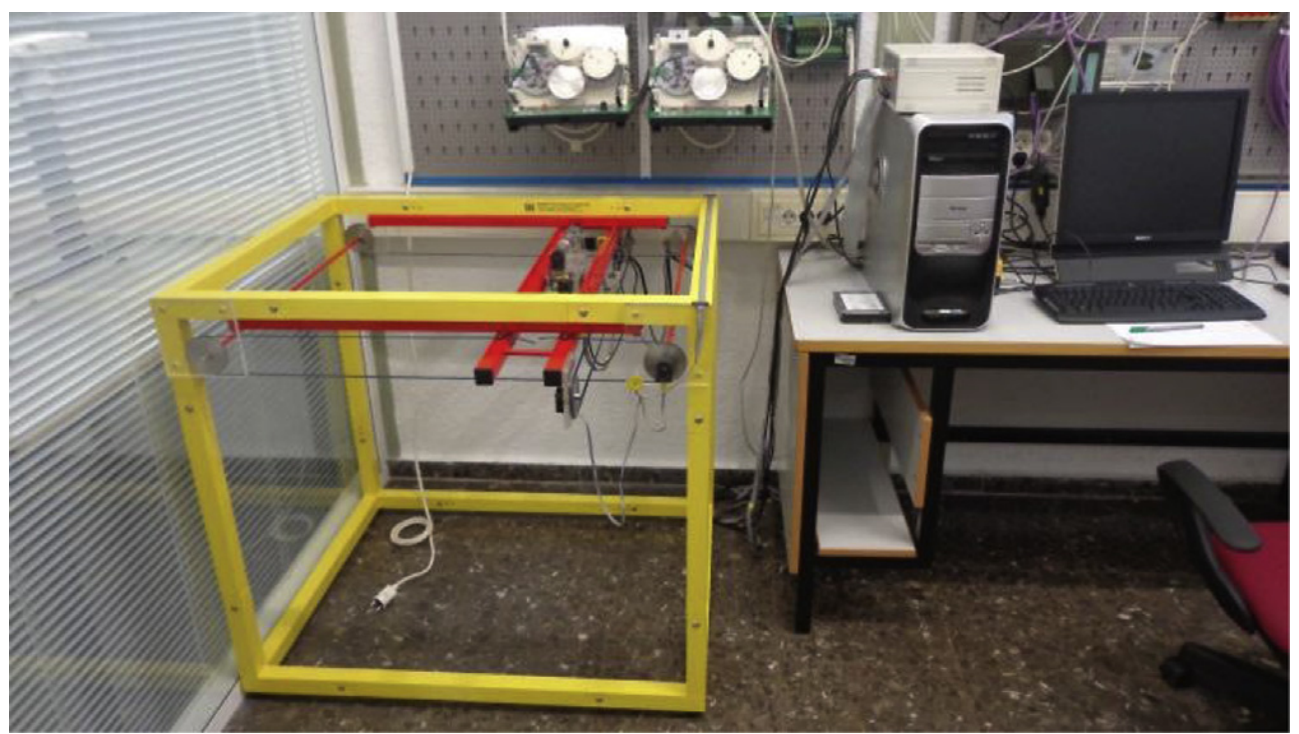

Figure 3.5: Cartesian robot (3D CRANE module)

The system also presents two non-linear behaviors to be taken into account in real-time implementation: saturation limits of control actions in \pm 1 , and dead zone values of \pm 0.06 . Both of them are identified experimentally and measured in normalized c.a.u.

In [20], a conventional PID controller such as in (3.6) is used with $K_{p}=12$, $T_{d}=0.01$ and $T_{i}=3.5$, which reaches certain specifications (null steady-state error, settling time around $4 \mathrm{~s}$, and overshot around $5 \%$ ). Digitally implementing this controller at a period higher than $0.1 \mathrm{~s}$, the aforementioned specifications cannot be assured. It is assumed that the sensor's nature or the network load do not allow a sampling period below $0.2 \mathrm{~s}$. Therefore, a sample time of $N T=0.2 s$ is used, and a dual-rate controller with $N=2$ is implemented using (3.7)-(3.17) (assuming $K_{P D}=K_{p}$ and $K_{P I}=1$ ) in order to reach the specifications. The following gain-scheduling law for the dual-rate delay-dependent controller is used to deal with the round-trip time delay $\tau_{k}$ :

$$
\begin{aligned}
& K_{P D}^{\tau_{k}}=-50 \tau_{k}+K_{P D} \\
& T_{d}^{\tau_{k}}=0.5 \tau_{k}+T_{d}
\end{aligned}
$$


For the present study, as enunciated in (3.2), a generalized exponential distribution is considered. The histogram used in this case is shown in Figure 3.6, where $\tau_{k}$ takes values in the range $\Theta=[0.04,0.08]$. In addition, as presented in (3.3), packet dropouts are modeled as a Bernoulli distribution, being in this case $p=p^{l r}=p^{r l}=0.3$ and $M=3$.

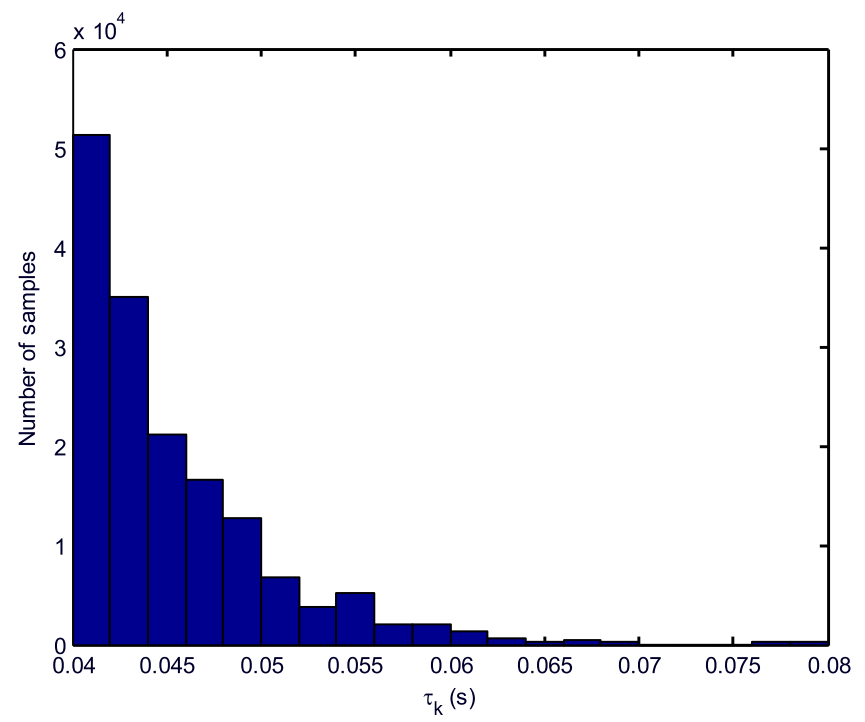

Figure 3.6: Delay histogram

In order to assess the stability of the setup in probabilistic time-varying delays, the LMI gridding in (3.29) has been carried out taking $l=20$ grid points so as to compute the closed-loop realizations $A_{c l, 0}\left(\vartheta_{j}\right)$ and $A_{c l, 1}\left(\vartheta_{j}\right)$ for the parameter space $\Theta$. From Figure 3.6, $P\left[\vartheta_{j}\right]$ is normalized in order to take values in $[0,1)$. As a result, it can be checked that a feasible solution $Q$ exists, which guarantees stability for the control system design:

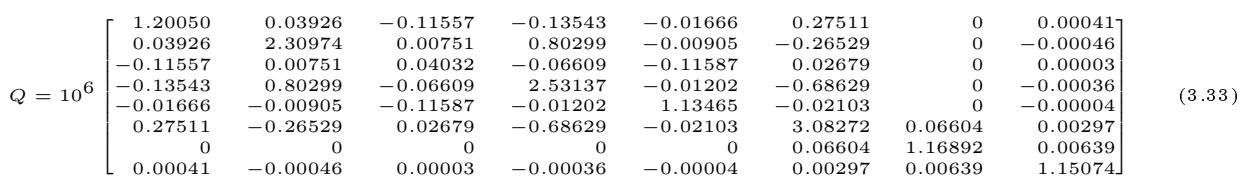

Figure 3.7 shows the results obtained for the control solution, where filtered step references are used in order to avoid the saturation of the control signal. Note that the sequence of packet dropouts is represented in the time axis in such a way that a point indicates a packet dropout in the time instant where it is plotted. If the point increases its value in the vertical axis, then consecutive 
dropouts are occurring in this instant. Figure 3.7 also shows the desired, nominal (no-delay, no-dropout) output. When packet dropouts appear in the NCS, if the delay-dependent control solution does not include the prediction stage, behavior deterioration is observed. Then, including the prediction stage, the control performance can be restored at about the same level as the nominal one.

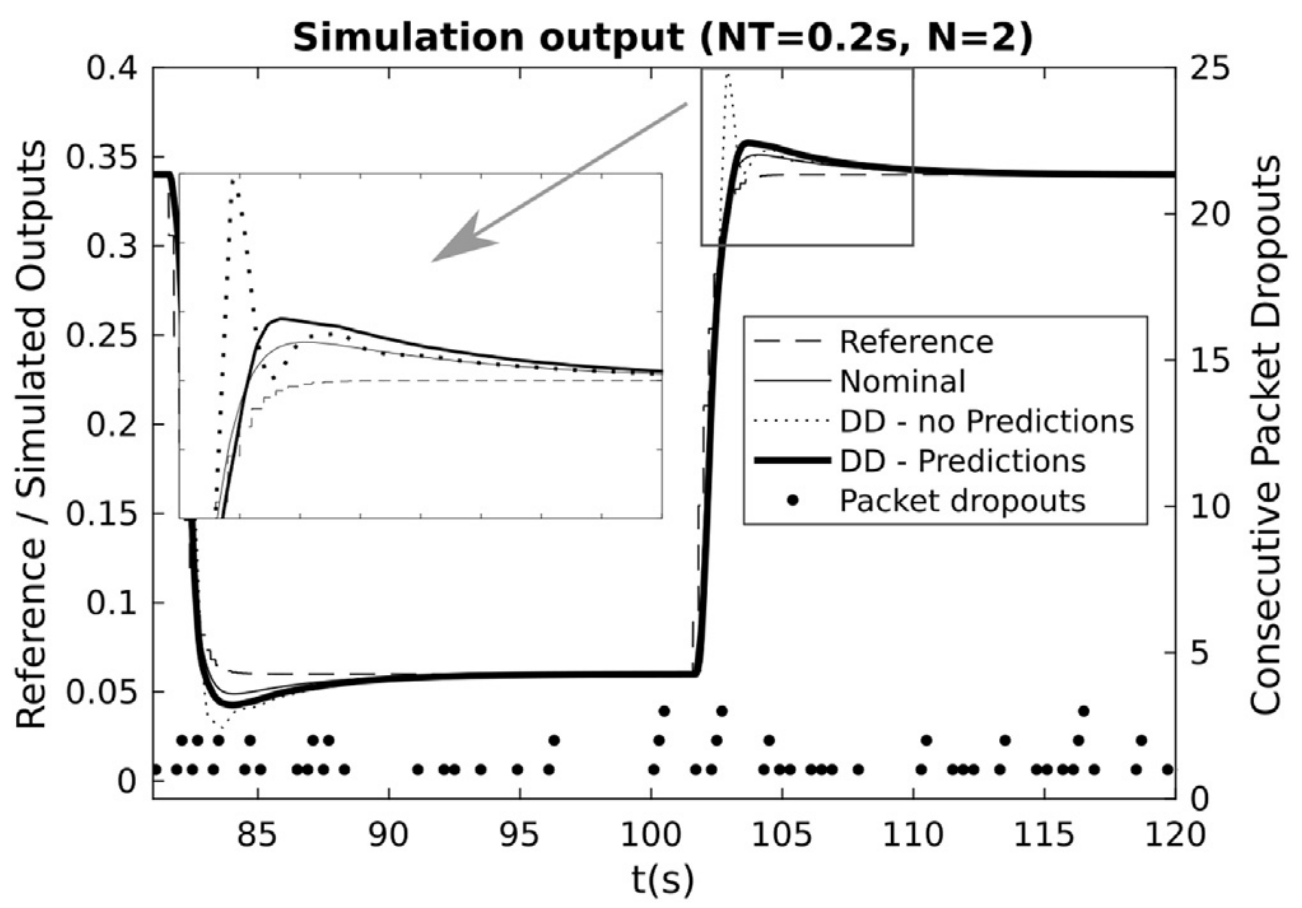

Figure 3.7: Simulation results: nominal vs delay-dependent with packet dropouts and no prediction vs delay-dependent with packet dropouts with prediction

\subsubsection{Data analysis via cost indexes}

In this subsection four different cost indexes will be used. Firstly, in order to better quantify the benefits of the packet-based control solution, the cost indexes $J_{1}$ and $J_{2}$ are utilized. The first one, $J_{1}$, is based on the Integral of Absolute Error (IAE), and the second one, $J_{2}$, on the overshoot value. $J_{1}$ and $J_{2}$ take the worst behavior, that is, the delay-dependent controller with no prediction stage as the reference value to be compared to the rest of behaviors in order to compute the consequent improvements. 
In order to define $J_{1}$ let us consider the array $Y$, which includes the different sequence of outputs to be analyzed for the dual-rate control system, that is, $Y=\left[Y_{N o m}, Y_{D D-N P}, Y_{D D-P}\right]$, being $Y_{N o m}$ the output for the nominal (no-delay, no-dropout) case, $Y_{D D-N P}$ the output for the delay-dependent controller with no prediction stage, and $Y_{D D-P}$ the output for the delay-dependent controller with prediction stage.

From $Y$ the following accumulated (integrated) error $E_{Y}$ in a range of time instants $\Gamma$ can be computed

$$
E_{Y}(i)=\sum_{\Gamma}\left|Y(i)-Y_{\text {Nom }}\right|, \quad i=1 . .3
$$

Then, the $J_{1}$ cost index takes this form

$$
J_{1}(i)=100-\frac{E_{Y}(i)}{E_{Y}(2)} 100(\%), \quad i=1 . .3
$$

being $E_{Y}(2)$ the worst expected accumulated error, that is, the error reached by the delay-dependent controller with no prediction stage. Therefore, the rest of the errors are measured by $J_{1}$ as an improvement (in \%) with regard to $E_{Y}(2)$.

In order to define $J_{2}$, from $Y$ the following overshoot $O_{Y}$ in a range of time instants $\Gamma$ can be calculated (considering positive -max- or negative -minfiltered step references)

$$
O_{Y}(i)=\max \left(\left|\max _{\Gamma} Y(i)-\max _{\Gamma} Y_{N o m}\right|,\left|\min _{\Gamma} Y(i)-\min _{\Gamma} Y_{N o m}\right|\right), \quad i=1 . .3
$$

Then, the $J_{2}$ index is

$$
J_{2}(i)=100-\frac{O_{Y}(i)}{O_{Y}(2)} 100(\%), \quad i=1 . .3
$$

being $O_{Y}(2)$ the worst expected overshoot, that is, the overshoot reached by the delay-dependent controller with no prediction stage. Similarly to $J_{1}$, the rest of the overshoots are measured by $J_{2}$ as an improvement (in \%) with regard to $O_{Y}(2)$. 


\begin{tabular}{|l|llll|}
\hline Output & $E_{Y}$ & $J_{1}(\%)$ & $O_{Y}$ & $J_{2}(\%)$ \\
\hline$Y_{D D-N P}$ & 291.19 & 0 & 0.047 & 0 \\
$Y_{D D-P}$ & 168.64 & 42.09 & 0.007 & 85.64 \\
$Y_{\text {Nom }}$ & 0 & 100 & 0 & 100 \\
\hline
\end{tabular}

Table 3.1: Cost indexes $J_{1}$ and $J_{2}$ in simulation

Table 3.1 summarizes the cost indexes obtained for each output. As expected, considering packet dropouts, the packet-based control strategy which includes prediction stage clearly improves the control performance obtained by the noprediction control strategy.

\subsection{Experimental results}

To validate the simulation results obtained in section 3.5, a laboratory test-bed platform is set up, which includes the CRANE module previously presented, two computers and an Ethernet cable. One computer is directly connected to the plant and composes the local part of the control system. The aims of this computer are: firstly, to be in charge of the measures on the plant at $N T=0.2 s$ and their communication; secondly, to be responsible for the reception of the slow-rate control signal from the PI controller, the calculation of the fast-rate PD control actions (at $T=0.1 s$ ) and their injection over the plant. When convenient, it is also in charge of the reception and selection of signals predicted to be applied to the plant when loss of communication occurs.

The second computer performs the remote part of the controller, receiving the outputs of the plant, calculating the slow-rate PI controller, and sending back these actions to the local system. When required, this part is also in charge of the calculation of future, slow, predicted control actions, which will be sent together with the slow-rate control signal.

These computers are connected by a UDP network through an Ethernet cable that performs the local-to-remote and remote-to-local links. In order to obtain similar conditions to those considered in simulation, network delays and packet dropouts are modified by software.

Figure 3.8 shows the outputs obtained in the experiment, which clearly reveals the same trend observed in Figure 3.7. To better validate the results, Table 3.2 details the cost indexes $J_{1}$ and $J_{2}$ computed for the experiment, where lower 


\begin{tabular}{|l|llll|}
\hline Output & $E_{Y}$ & $J_{1}(\%)$ & $O_{Y}$ & $J_{2}(\%)$ \\
\hline$Y_{D D-N P}$ & 290.55 & 0 & 0.042 & 0 \\
$Y_{D D-P}$ & 207.53 & 28.58 & 0.012 & 70.80 \\
$Y_{\text {Nom }}$ & 0 & 100 & 0 & 100 \\
\hline
\end{tabular}

Table 3.2: Cost indexes $J_{1}$ and $J_{2}$ in experimentation

improvements than in simulation are achieved due to practical issues (possible divergences between model and plant, dead zone, and so on).

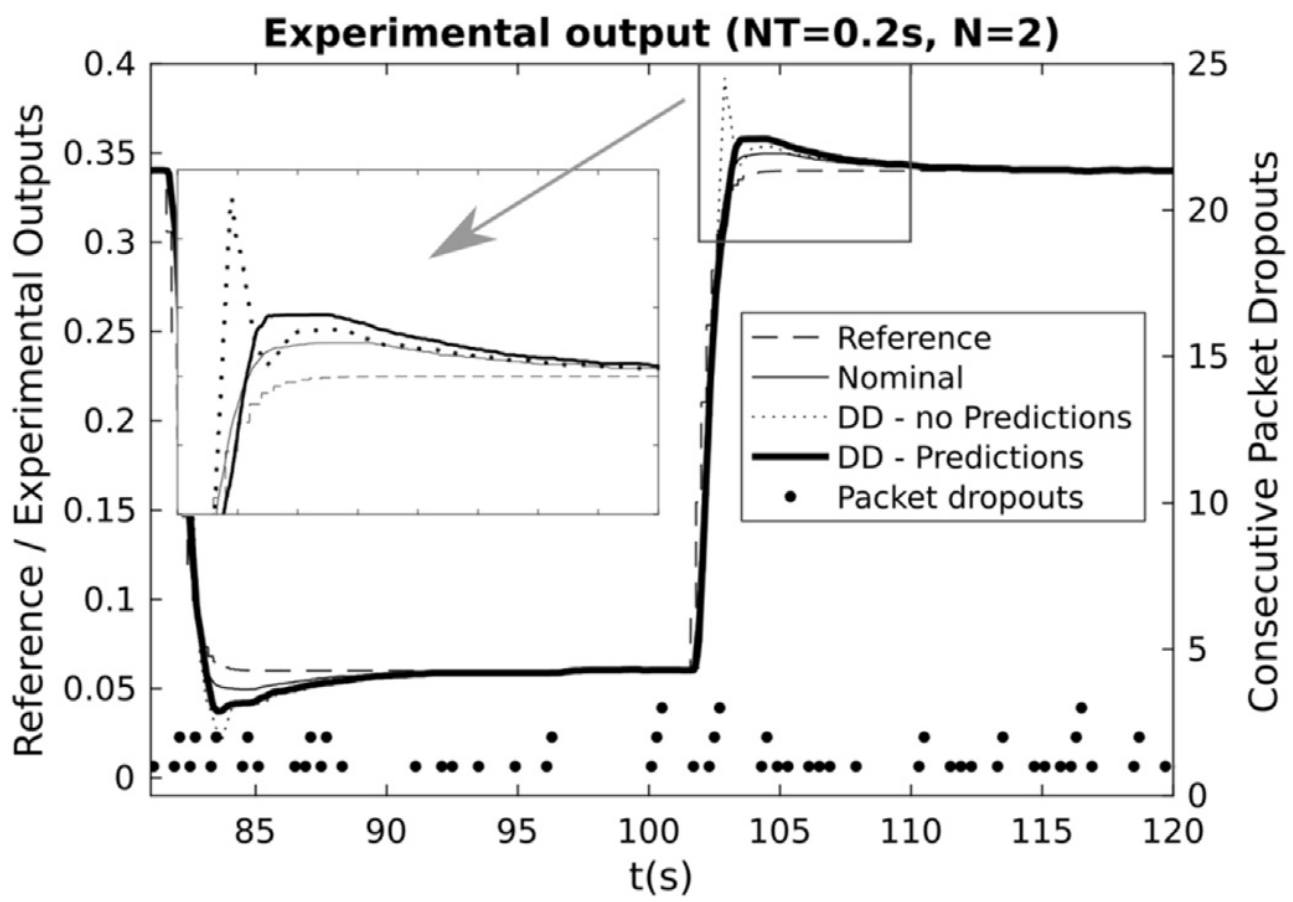

Figure 3.8: Experimental results: nominal vs delay-dependent with packet dropouts and no prediction vs delay-dependent with packet dropouts with prediction 


\subsection{Conclusions}

In this work, a packet-based dual-rate control solution is presented for an NCS where time-varying delays, packet dropouts and packet disorder can occur. Selecting the sensing period greater than the longest round-trip time delay, the packet disorder is avoided. In addition, energy saving can be achieved by reducing network load, which is a crucial issue, especially in battery-powered wireless sensors. However, in order to reach certain specifications, an $N$ times faster actuation period must be used, leading to the dual-rate control structure. The time-varying delays are dealt with the gain scheduling approach in [20]. By means of the packet-based control strategy, and including a prediction stage which is able to treat unstable or marginally stable plants, the packet dropout problem is solved. Control system stability is ensured via probabilistic LMIs.

Simulation results reveal the improvements reached by the control proposal, which are validated by means of a laboratory test-based platform, where the process to be controlled is a Cartesian robot.

\section{Acknowledgements}

This work is funded by European Commission as part of Project H2020-SEC2016-2017 - Topic: SEC-20-BES-2016 - Id: 740736 - "C2 Advanced Multidomain Environment and Live Observation Technologies" (CAMELOT). Part WP5 supported by Tekever ASDS, Thales Research \& Technology, Viasat Antenna Systems, Universitat Politècnica de València, Fundação da Faculdade de Ciências da Universidade de Lisboa, Ministério da DefesaNacional e Marinha Portuguesa, Ministério da Administração Interna Guarda Nacional Republicana. 



\section{References}

[1] Xian-Ming Zhang, Qing-Long Han, and Xinghuo Yu. "Survey on Recent Advances in Networked Control Systems". In: IEEE Transactions on Industrial Informatics 12.5 (Oct. 2016), pp. 1740-1752. ISSN: 1551-3203. DOI: 10.1109/TII.2015.2506545 (cit. on pp. 56, 61).

[2] Dan Zhang et al. "Analysis and synthesis of networked control systems: A survey of recent advances and challenges". In: ISA Transactions 66 (2017), pp. 376-392. ISSN: 0019-0578. DOI: https://doi.org/10.1016/ j.isatra.2016.09.026 (cit. on p. 56).

[3] J. Qiu, H. Gao, and M. Chow. "Networked control and industrial applications [Special section introduction]". In: IEEE Transactions on Industrial Electronics 63.2 (Feb. 2016), pp. 1203-1206. ISSN: 0278-0046. DOI: 10.1109/TIE. 2015.2506544 (cit. on p. 56).

[4] Behrooz Rahmani, Amir H.D. Markazi, and Behzad Seyfi. "A new method for control of networked systems with an experimental verification". In: ISA Transactions 56 (2015), pp. 299-307. ISSN: 0019-0578. DOI: https: //doi.org/10.1016/j. isatra.2014.12.004 (cit. on p. 56).

[5] Geng Liang. "Control and communication co-design: Analysis and practice on performance improvement in distributed measurement and control system based on fieldbus and Ethernet". In: ISA Transactions 54 (2015), pp. 169-192. ISSN: 0019-0578. DOI: https : // doi .org/10.1016/j . isatra.2014.08.012 (cit. on p. 56).

[6] Carlos Santos et al. "Event-Based Sensing and Control for Remote Robot Guidance: An Experimental Case". In: Sensors 17.9 (2017). ISSN: 14248220. DOI: $10.3390 /$ s17092034 (cit. on p. 56).

[7] R. Pizá et al. "Hierarchical Triple-Maglev Dual-Rate Control Over a Profibus-DP Network". In: IEEE Transactions on Control Systems Technology 22.1 (Jan. 2014), pp. 1-12. ISSN: 1063-6536. DOI: 10.1109/TCST. 2012.2222883 (cit. on p. 56). 
[8] Daniel Georgiev and Dawn M. Tilbury. "Packet-based control: The H2optimal solution". In: Automatica 42.1 (2006), pp. 137-144. ISSN: 00051098. DOI: https://doi .org/10.1016/j . automatica . 2005.08.011 (cit. on p. 56).

[9] Yun-Bo Zhao, Guo-Ping Liu, and David Rees. "Design of a Packet-Based Control Framework for Networked Control Systems". In: IEEE Transactions on Control Systems Technology 17.4 (July 2009), pp. 859-865. ISSN: 1063-6536. DOI: 10.1109/TCST . 2008.2010946 (cit. on pp. 56, 73).

[10] Yun-Bo Zhao, Guo-Ping Liu, and David Rees. "Modeling and Stabilization of Continuous-Time Packet-Based Networked Control Systems". In: IEEE Transactions on Systems, Man, and Cybernetics, Part B (Cybernetics) 39.6 (Dec. 2009), pp. 1646-652. ISSN: 1083-4419. DOI: 10.1109/ TSMCB. 2009. 2027714 (cit. on p. 56).

[11] Yun-Bo Zhao et al. "Packet-Based Control Design for Networked Control Systems". In: Packet-Based Control for Networked Control Systems. Springer, 2017, pp. 15-32 (cit. on p. 56).

[12] W. P. M. H. Heemels, M. C. F. Donkers, and A. R. Teel. "Periodic EventTriggered Control for Linear Systems". In: IEEE Transactions on Automatic Control 58.4 (Apr. 2013), pp. 847-861. ISSN: 0018-9286. DOI: 10.1109/TAC.2012.2220443 (cit. on p. 56).

[13] Miguel G. Villarreal-Cervantes et al. "Stabilization of a $(3,0)$ mobile robot by means of an event-triggered control". In: ISA Transactions 58 (2015), pp. 605-613. ISSN: 0019-0578. DOI: https : //doi.org/10.1016/j . isatra.2015.06.013 (cit. on p. 56).

[14] Vicente Casanova et al. "Control of the rotary inverted pendulum through threshold-based communication". In: ISA Transactions 62 (2016). SI: Control of Renewable Energy Systems, pp. 357-366. ISSN: 0019-0578. DOI: https://doi.org/10.1016/j.isatra.2016.01.009 (cit. on p. 56).

[15] Joa-Hyoung Lee and In-Bum Jung. "Adaptive-Compression Based Congestion Control Technique for Wireless Sensor Networks". In: Sensors 10.4 (2010), pp. 2919-2945. ISSN: 1424-8220. DOI: 10.3390/s100402919 (cit. on p. 56).

[16] Wei Chen and Li Qiu. "Stabilization of networked control systems with multirate sampling". In: Automatica 49.6 (2013), pp. 1528-1537. ISSN: 0005-1098. DOI: https://doi.org/10.1016/j .automatica. 2013.02 . 010 (cit. on p. 56). 
[17] Julián Salt et al. "A Multirate Control Strategy to the Slow Sensors Problem: An Interactive Simulation Tool for Controller Assisted Design". In: Sensors 14.3 (2014), pp. 4086-4110. ISSN: 1424-8220. DOI: 10.3390/ s140304086 (cit. on pp. 56, 57, 60, 62, 66).

[18] Antonio Sala, Ángel Cuenca, and Julián Salt. "A retunable PID multirate controller for a networked control system". In: Information Sciences 179.14 (2009), pp. 2390-2402. ISSN: 0020-0255. DOI: https://doi.org/ 10.1016/j.ins.2009.02.017 (cit. on pp. 57, 71, 72).

[19] Ángel Cuenca et al. "A Delay-Dependent Dual-Rate PID Controller Over an Ethernet Network". In: IEEE Transactions on Industrial Informatics 7.1 (Feb. 2011), pp. 18-29. ISSN: 1551-3203. DOI: 10.1109/TII . 2010. 2085007 (cit. on pp. 57, 73).

[20] Angel Cuenca et al. "An approach based on an adaptive multi-rate smith predictor and gain scheduling for a networked control system: Implementation over Profibus-DP". In: International Journal of Control, Automation and Systems 8.2 (Apr. 2010), pp. 473-481. ISSN: 2005-4092. DOI: 10.1007/s12555-010-0237-1 (cit. on pp. 57, 58,63,67, 75, 81).

[21] Jean-Chrysotome Bolot. "End-to-end packet delay and loss behavior in the Internet". In: ACM SIGCOMM Computer Communication Review. Vol. 23. 4. ACM. 1993, pp. 289-298 (cit. on pp. 57, 61).

[22] Amarnath Mukherjee. "On the dynamics and significance of low frequency components of Internet load". In: Technical Reports (CIS) Dep. of Computer 8 Information Science. Univ. Pennsylvania. 1992 (cit. on pp. 57,60).

[23] S. Mondie, R. Lozano, and J. Collado. "Resetting process-model control for unstable systems with delay". In: Proceedings of the 40th IEEE Conference on Decision and Control (Cat. No.01CH37228). Vol. 3. Dec. 2001, 2247-2252 vol.3. DOI: 10.1109/CDC. 2001.980591 (cit. on pp. 58, $69,70)$.

[24] S. Mondié, P. Garcia, and R. Lozano. "Resetting Smith predictor for the control of unstable systems with delay". In: IFAC Proceedings Volumes 35.1 (2002). 15th IFAC World Congress, pp. 77-82. ISSN: 1474-6670. DOI: https://doi.org/10.3182/20020721-6-ES-1901.00581 (cit. on pp. 58, $69,70)$.

[25] Y. Tipsuwan and Mo-Yuen Chow. "Gain scheduler middleware: a methodology to enable existing controllers for networked control and teleoperation - part I: networked control". In: IEEE Transactions on Industrial Electronics 51.6 (Dec. 2004), pp. 1218-1227. ISSN: 0278-0046. DOI: 10.1109/TIE. 2004.837866 (cit. on p. 60). 
[26] P. Khargonekar, K. Poolla, and A. Tannenbaum. "Robust control of linear time-invariant plants using periodic compensation". In: IEEE Transactions on Automatic Control 30.11 (Nov. 1985), pp. 1088-1096. ISSN: 0018-9286. DOI: 10.1109/TAC.1985.1103841 (cit. on p. 71).

[27] S. Boyd et al. Linear Matrix Inequalities in System and Control Theory. Studies in Applied Mathematics. Society for Industrial and Applied Mathematics (SIAM, 3600 Market Street, Floor 6, Philadelphia, PA 19104), 1994. ISBN: 9781611970777 (cit. on p. 73). 


\title{
Chapter 4
}

\section{Paper 3. Dual-rate sampled-data systems. Some interesting consequences from its frequency response analysis}

\author{
Julián Salt, José Alcaina
}

The main goal of this contribution is to introduce a new procedure in order to analyze properly SISO dual-rate systems (DRS) and to provide straightforward answers to some common general questions about this kind of systems. Frequency response analysis based on DRS lifting modeling can lead to interesting results about stability margins or performance prediction. As a novelty, it is explained how to understand DRS frequency response and how to handle it for an easy computation of magnitude and phase margins keeping classical frequency domain methods. There are also some repetitive questions about DRS that can be analyzed and answered properly using the results from this contribution: what the optimum relation between sampling periods is or what effects does delay have in a DRS. Every step is illustrated with examples that should clarify the understanding of the text.

Keywords: Dual-rate systems; frequency response; stability; Bode diagram.

International Journal of General Systems, volume 48 - issue 5, apr 2019, pages 554-574, https://doi. org/10.1080/03081079. 2019. 1608984 


\subsection{Motivation}

In general a multi-rate sampled-data system (MRS) is defined as a hybrid system composed of continuous time elements, usually the plant, and some discrete time components, usually the controllers and/or the filters, where two or more variables are sampled or updated at different frequencies. These systems have been reported and applied in fields like signal processing and communications for a long time. In digital control systems MRS were assumed by engineers since the fifties with seminal papers like [1-4]. When there are just two sampling periods, the system is called dual-rate. The consideration of this kind of systems is proposed mainly due to restrictions in sampling periods. For instance slow chemical measurements or artificial vision sensing where the processing data is time consuming. This situation leads to unacceptable sampling times for control purposes in a single rate environment. In networked control systems (NCS) it is a viable solution either to avoid delays [5] or to save network load by sending less information across the shared medium. Due to the current importance of the NCS applications, other non-conventional sampling schemes (like DRS) for dealing with communication bandwidth restrictions, such as event-triggered sampling, have been lately proposed [6-9]. In some low cost devices, DRS is also necessary for solving problems due to the low resolution of sensors (cheap encoders) [10]. These DRS cases are what is defined as MRIC (multi-rate input control) that is a slow output but a higher rate input which is the most common schema in DRS. It is usual that both sampling periods are related by a natural number $N \in \mathbb{N}$. A regular pattern of sampled signals without sampling times mismatch between them will also be considered.

In the field of control systems, there are two trends for modelling MRS (and DRS). It can be said that one of them is an external representation based on [11-14] and the other one is a state space form. These options consider the least common multiple of all sampling periods for modelling purposes ${ }^{1}$. In the external case the skip (downsampling) and expand (upsampling) operations are crucial and in the state space form a MIMO model is assumed enveloping the input and output of every system in vectors according to the frame period (least common multiple). The system progresses repeating the same scheme frame after frame. Therefore this MR (time-varying system) can be transformed into a LTI system once the system description is enlarged over a "metaperiod". Using this technique, even from a single-input single-output (SISO) system an artificial multi-input and multi-output (MIMO) system is obtained. This procedure is known as lifting in control area [15-17], originally denoted as Vectorial Switch Decomposition by [1] or blocking (in signal processing) [18].

\footnotetext{
${ }^{1} \mathrm{~A}$ more formal definition will be given in section 4.2
} 
Consider now a SISO system with two samplers (DRS); in these conditions lifting provides a MIMO system. A classical issue in multi-rate control schemes is that such a MIMO lifted system can be managed as any other multivariable MIMO one. However, there are not different input and output variables, but just one input and one output, "lifted" at different input and output sampling times in a periodically-repeating metaperiod. This will be a very important aspect when a DRS frequency response is considered. Usually classical MIMO techniques are considered -it is common to study the frequency response of dual-rate plants by using singular value decomposition (SVD) of the lifted MIMO system-, losing important features that will be introduced in this contribution. In order to solve this problem a new algorithm for DRS frequency response computation in discrete control systems was introduced by [19], but it seems difficult to understand the complete meaning from that reference, which will be clarified now. It has been studied how this technique can be advantageous by obtaining the frequency response and its derived concepts and procedures when applying to DRS. In fact, this is the main goal of this article and some examples are introduced to emphasize its importance. Specifically it will attempt to meet answers to repetitive questions made when a DRS is analyzed: what is the best relation between sampling periods or about the influence of delay in these systems.

The structure of the paper is as follows: the next section introduces some preliminary material and exposes how to compute the frequency response for a DRS in an efficient way; section 4.3 presents how to make this frequency response easily understandable introducing an example. In the following sections, the results from the previous section are used to explain the advantages of DRS frequency response and how to answer some classical questions about the optimum value of $N$ in a simple MRIC DRS. A conclusion section closes the paper.

\subsection{DRS frequency response computation: a classical interpretation}

\subsubsection{Preliminaries and notation}

A dual-rate discrete LTI system is one in which the input and output sequences are assumed to have different sampling periods, $T_{u}$ and $T_{y}$. If they are rationally related, it is possible to define $T_{0}$ as the minimum real number such that $T_{0} / T_{u}$ and $T_{0} / T_{y}$ are integers. It is similar to a least common multiple $T_{0}=\operatorname{lcm}\left(T_{u}, T_{y}\right)$ whose definition related to real numbers can be confusing. 
$T_{0}$ is usually known as "metaperiod" or "frame period" and integers $N_{u}, N_{y}$ can be defined complying with $T_{0}=T_{u} N_{u}=T_{y} N_{y}$ (indeed, then $T_{u} / T_{y}=N_{y} / N_{u}$ is a rational number). It is also usual to define $T$ as a kind of "greatest common divisor sampling period", such that $T_{0}=N T$ being $N=\operatorname{lcm}\left(N_{u}, N_{y}\right)$; therefore $T_{0}=N T=N_{u} T_{u}=N_{y} T_{y}$. With these conditions, the behavior of the DRS may be characterized via a "lifted" transfer function matrix

$$
y_{l}\left(z^{N}\right)=G_{l i f t e d}\left(z^{N}\right) u_{l}\left(z^{N}\right)
$$

where the variable $z$ stands for the LTI $z$-transform argument at sampling period $T$, and consequently $z^{N}$ is related to $T_{0}=N T$. In equation (4.1) $y_{l}$ is a vector of length $N_{y}, u_{l}$ is a vector of length $N_{u}$ and $G_{l i f t e d}$ is a $N_{y} \times N_{u}$ transfer function matrix [15]. The lengths of the vectors are increased in the case of MIMO systems (multiplied by the number of outputs and inputs, respectively). The next figures can help to completely deduce and clarify this equation.

Figure 4.1 depicts an open loop DRS and Figure 4.2 describes the application of Kranc's idea vector switch decomposition to the situation of Figure 4.1.

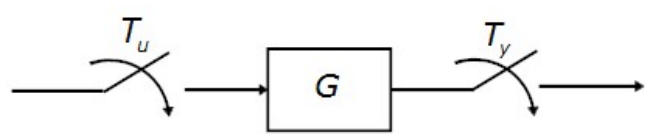

Figure 4.1: Generalized Dual-Rate System

Note that in Figure 4.2, $T_{0} / N_{u}=N_{y} T$ and $T_{0} / N_{y}=N_{u} T$. It can be seen that with this scheme, the central part of the diagram would be

$$
\left(\begin{array}{llll}
1 & z^{-N_{u}} & \ldots & z^{-\left(N_{y}-1\right) N_{u}}
\end{array}\right) \frac{G_{l i f t e d}\left(z^{N_{y} N_{u}}\right)}{N_{y}}\left(\begin{array}{c}
1 \\
z^{N_{y}} \\
\vdots \\
z^{\left(N_{u}-1\right) N_{y}}
\end{array}\right)
$$

This expression can be also derived using the known Mason's rule for blocks diagram. This diagram is now a $T_{0}$ single rate blocks diagram ${ }^{2}$. Actually, that is the main reason for Kranc's procedure.

\footnotetext{
${ }^{2}$ It will denoted $w_{s}=2 \pi / T_{0}$
} 


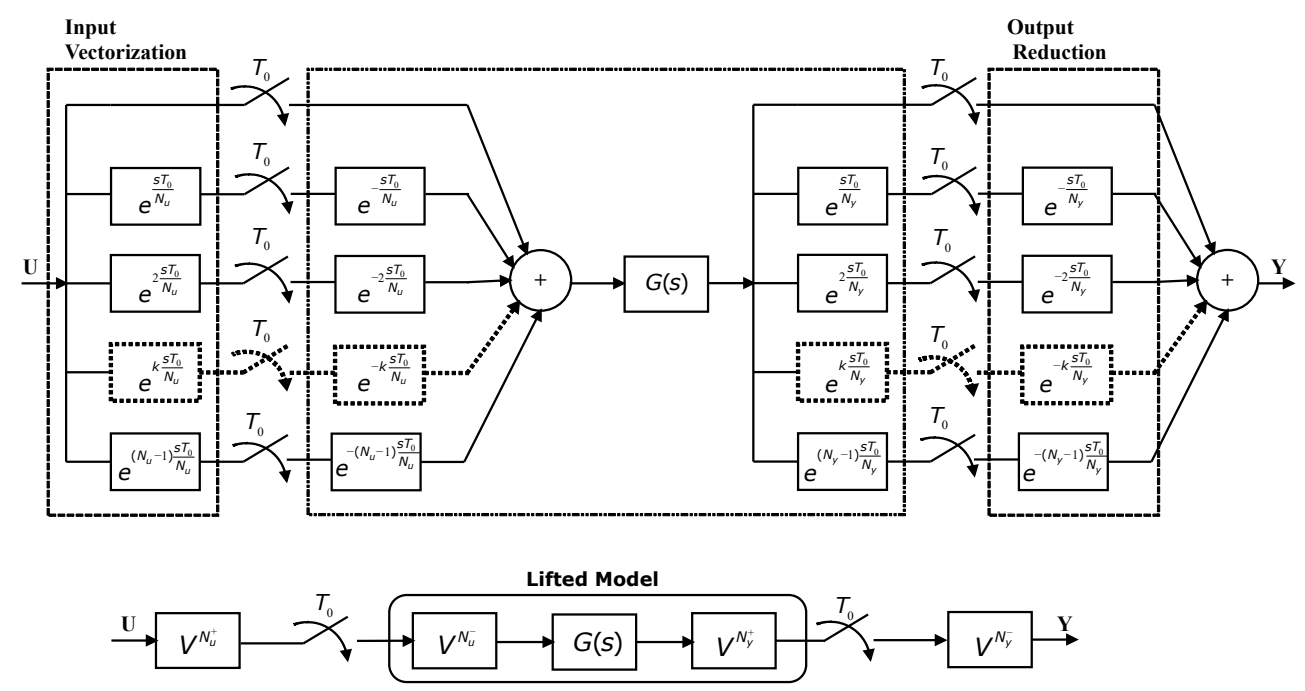

Figure 4.2: Kranc's vector switch decomposition

\subsubsection{Sampled-data lifted systems}

There are different ways to obtain the lifted model. For processes, it may be that the easiest one is to consider the successive iterations from the gcd $T$ discrete state space. If a strictly proper continuous system is discretized (assuming $\mathrm{ZOH}$ ) at period $T$, with a realization $(A, B, C, 0)$, then the lifted dual-rate model has a realization $\left(A_{l}, B_{l}, C_{l}, D_{l}\right)$, at metaperiod $T_{0}$, where these matrices are obtained by repeated evaluations of the equations at sampling period $T$ giving rise to well-known convolution-like formulae. For instance

$$
\begin{aligned}
y\left(k T_{0}+\zeta T\right)= & C x\left(k T_{0}+\zeta T\right)= \\
& =C\left[A^{\zeta} x\left(k T_{0}\right)+A^{\zeta-1} B u\left(k T_{0}\right)+\right. \\
& \left.+A^{\zeta-2} B u\left(k T_{0}+T\right)+\cdots+B u\left(k T_{0}+(\zeta-1) T\right)\right]
\end{aligned}
$$

for $\zeta=1, \ldots,\left(N_{u}-1\right) N_{y}$. However, the zero-order-hold entails

$$
\begin{aligned}
& u\left(k T_{0}+d N_{y} T\right)=u\left(k T_{0}+\left(d N_{y}+1\right) T\right)=\cdots=u\left[k T_{0}+\left((d+1) N_{y}-1\right) T\right] \\
& \forall d=0,1 \ldots,\left(N_{u}-1\right)
\end{aligned}
$$


Therefore the lifted matrices are obtained by suitably stacking the results from the above equation. It is also possible to consider that $\mathrm{G}$ has different natures (continuous system without ZOH, digital system, etc). For each of these cases a proper input or output assignment (like (4.4)) must be established leading to a specific lifted model [20]. Some other details, omitted here for brevity, can be found in $[15,17,21]$. Now, a brief example could be considered. If in Figure $4.1 \mathrm{G}$ is a continuous-time plant with $\mathrm{ZOH}$ and $T_{u}=0.3$ y $T_{y}=0.2$ $\left(N_{u}=2\right.$ and $\left.N_{y}=3\right)$, then the $1 \mathrm{~cm} T_{0}=0.6$, the $\operatorname{gcd} T=0.1$, and the lifted representation will be

$$
\begin{gathered}
x\left[(k+1) T_{0}\right]=A^{6} x\left(k T_{0}\right)+\left(\begin{array}{ll}
\left(A^{5}+A^{4}+A^{3}\right) B & \left(A^{2}+A+I\right) B
\end{array}\right)\left(\begin{array}{c}
u\left(k T_{0}\right) \\
u\left(k T_{0}+3 T\right)
\end{array}\right) \\
\left(\begin{array}{c}
y\left(k T_{0}\right) \\
y\left(k T_{0}+2 T\right) \\
y\left(k T_{0}+4 T\right)
\end{array}\right)=\left(\begin{array}{c}
C \\
C A^{2} \\
C A^{4}
\end{array}\right) x\left(k T_{0}\right)+\left(\begin{array}{cc}
0 & 0 \\
C(A+I) B & 0 \\
C\left(A^{3}+A^{2}+A\right) B & C B
\end{array}\right)\left(\begin{array}{c}
u\left(k T_{0}\right) \\
u\left(k T_{0}+3 T\right)
\end{array}\right)
\end{gathered}
$$

\subsubsection{DRS Frequency Response}

From a lifted LTI representation of a DRS, it is possible to calculate the frequency response. The Theorem introduced in [19] says: The output $y(k)$, when $u(k)=e^{j \omega T_{u} k}$, of a SISO dual-rate $\left(N_{u} T_{u}=N_{y} T_{y}\right)$ lifted system $y_{l}(z)=$ $G_{\text {lifted }}(z) u_{l}(z)$ is a collection of components $y_{r}(k)=\bar{y}_{r} e^{j T_{y} \omega_{r} k}$ of frequencies $\omega_{r}=\omega+2 \omega_{y}^{s} r / N_{y}$, for $r=0, \ldots,\left(N_{y}-1\right)$, with $\omega_{y}^{s}=\pi / T_{y}$, and $\bar{y}_{r}$ is given by

$$
\bar{y}_{r}=\frac{1}{N_{y}} \sum_{p=0}^{N_{y}-1} \sum_{q=0}^{N_{u}-1} G_{p q}\left(e^{j \omega_{r} T_{y} N_{y}}\right) e^{-j T_{y} \omega_{r} p} e^{j \omega T_{u} q}
$$

The singe-rate discrete frequency-response computations are carried out by replacing $z=e^{j \omega T}$ for some $T$. It is easy to check that in the DRS case, from (4.6), the components will be given by the product of the frequency response of the left factor

$$
\left[1 z^{-1} z^{-2} \ldots z^{-\left(N_{y}-1\right)}\right] G_{l i f t e d}\left(z^{N_{y}}\right)
$$

replacing $z=e^{j \omega_{r} T_{y}}$, which gives a row vector, and the right factor (column vector)

$$
\left(1 z z^{2} \ldots z^{N_{u}-1}\right)^{T}
$$


replacing $z=e^{j \omega T_{u}}$.

As it will be explained later, there is a possibility of computing the whole frequency response from only one Bode plot in the case in which $N_{y}$ and $N_{u}$ are coprime. Note that, $\omega_{s}=2 \pi /\left(N_{y} T_{y}\right)$ and then that the components of the frequency response are defined at $\omega_{r}=\omega+r \omega_{s}$, for, $r=0, \cdots, N_{y}-1$. For more details, see [19].

\subsection{Frequency Response Interpretation}

As it is relatively easy to understand considering the equation (4.2) and unwrapping it, that is, doing the matrices products, an usually high dimension SISO transfer function is reached. This is a simple procedure but it produces very unsatisfactory results as will be shown later in this section. The results from the algorithm proposed in the previous section are proper but require an interpretation. From the results above, it can be tested that the DRS frequency response can be explained by adding $\frac{N_{y}}{\operatorname{gcd}\left(N_{u}, N_{y}\right)}$ values from the Bode diagram outlined from 0 to $\left(\frac{N_{y}}{g c d\left(N_{u}, N_{y}\right)}-1\right) N_{u} w_{s}$. In fact, for an input frequency $b \operatorname{Rad} / \mathrm{s}$, the output is the sum of sine signals with frequencies $b, b+N_{u} w_{s}, b+2 N_{u} w_{s}$, $\cdots b+\left(\frac{N_{y}}{\operatorname{gcd}\left(N_{u}, N_{y}\right)}-1\right) N_{u} w_{s}$ with amplitude and phase determined by Bode diagram at the indicated points. The coprime case is illustrative and gives a link with the theoretical results. If $N_{u}$ and $N_{y}$ are coprime, then, considering the blocks diagram from Figure 4.2, the DRS frequency response is obtained reading the $N_{y}$ points from the magnitude and phase Bode diagrams in the interval from 0 to $N_{u} N_{y} w_{s}$, each length $N_{u} w_{s}$.

The proposed algorithm introduced in [19] returns $N_{y}$ Bode diagrams concerning to the tracks: 0 to $N_{u} N_{y} w_{s}, N_{u} w_{s}$ to $\left(N_{y} N_{u}+N_{u}\right) w_{s}, \cdots,\left(N_{y}-1\right) N_{u} w_{s}$ to $\left(N_{y} N_{u}+\left(N_{y}-1\right) N_{u}\right) w_{s}$. So, the same Bode translated $N_{u} w_{s} R a d / s N_{y}$ times is reached. Of course, the $N_{y}$ points can be read in the interval 0 to $N_{u} w_{s}$ knowing the true sense of all of them.

In the following section, a simple example is introduced illustrating the procedure. The academic system $G(s)=\frac{1}{(s+1)(s+4)}$ preceded by a zero order hold is considered, with $N_{u}=2$ and $N_{y}=3$ and a metaperiod $T_{0}=1$, that is $T_{u}=1 / 2$ and $T_{y}=1 / 3$. The DRS frequency response is formed by three components. The result is showed in Figure 4.3.

For this case, $w_{s}=\frac{2 \pi}{T_{0}}=6.28 R / s, N_{u} w_{s}=12.566 R / s$ and $N_{u} N_{y} w_{s}=$ $37.699 R / s$. As it can be seen, the Bode diagram is made up of three com- 

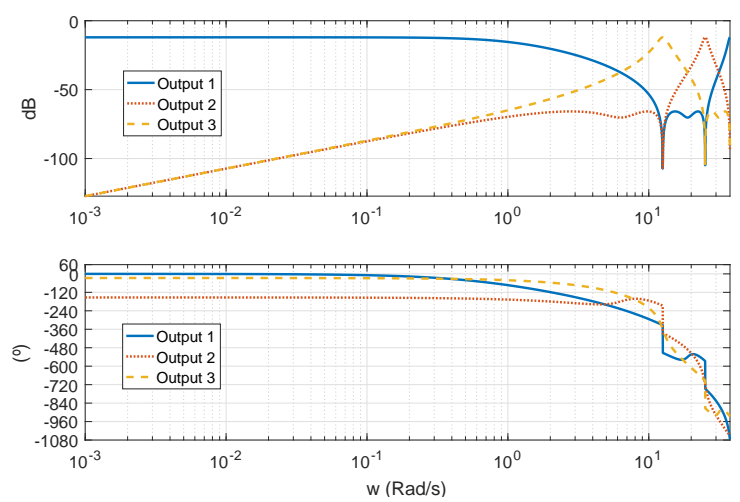

Figure 4.3: DRS Bode

ponents whose responses are achieved each $N_{u} w_{s}$. In fact and as it was said before, if the input is a sine signal with pulsation $w=3 R / s$, the three components are:

\begin{tabular}{|l|lll|}
\hline Component & $\mathrm{w}(\mathrm{R} / \mathrm{s})$ & Magnitude $(\mathrm{dB})$ & Phase $(\mathrm{Rad})$ \\
\hline 1 & 3 & -24.8 & -2.64 \\
2 & $3+12.56=15.56$ & -65.85 & 2.96 \\
3 & $3+(2 * 12.56)=28.3$ & -51.11 & -1.275 \\
\hline
\end{tabular}

In Figure 4.4 and Figure 4.5 the procedure is explained graphically. If the multiplicities $N_{u}, N_{y}$ are coprime it is possible to read every value in just one Bode diagram. This is the same as reading the value of each component in its proper $w$ and it is also the same as reading the 0 to $w_{s}$ zone but taking into account that the real value of the frequency of component $i$ is $w+i \times N_{u} w_{s}$. Note the linear scale of the frequency axis in both figures.

At this point, it is time to check the accuracy of the procedure. Adding the three responses, the result is equivalent to the output of the $Z O H-G(s)$ with the proposed input.

As it can be seen in Figure 4.6 the output true response is mapped at $T_{y}$ at steady state.

However, in the classical way, as it was said before, the problem is the high dimension of the transfer function that usually leads to an inefficient use of 

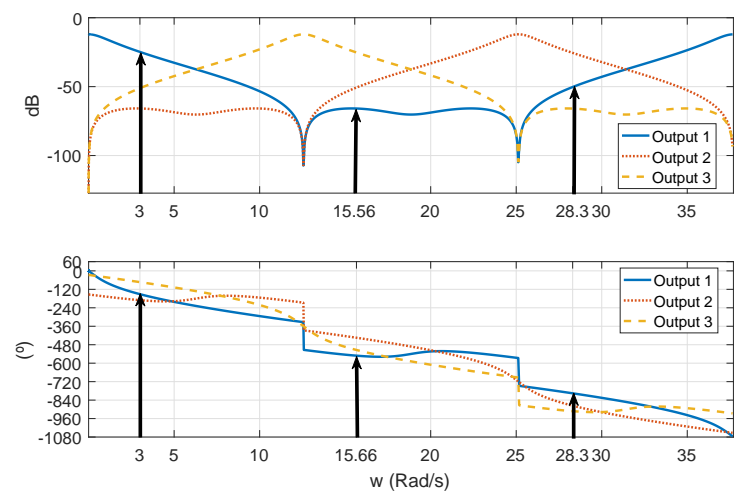

Figure 4.4: DRS Bode Interpretation. $w=3 R / s$
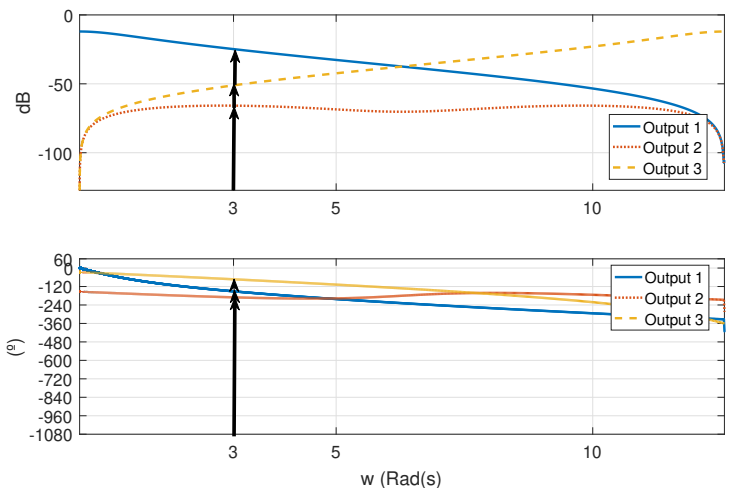

Figure 4.5: DRS Bode. $w=3 R / s$. Zoom

classical Bode computation routines. Some examples are now introduced for the explanation of this problem. If the continuous-time system with $\mathrm{ZOH}$

$$
G(s)=\frac{20}{(s+1)(s+3)}
$$

is considered, the results for a metaperiod $T_{0}=0.1 T_{u}=T_{0} / 2$ and $T_{y}=T_{0} / 3$ computed using both methods are plotted in Figure 4.7. As it is observed, the results from the procedure based on obtaining the transfer function show some differences with the correct ones. 


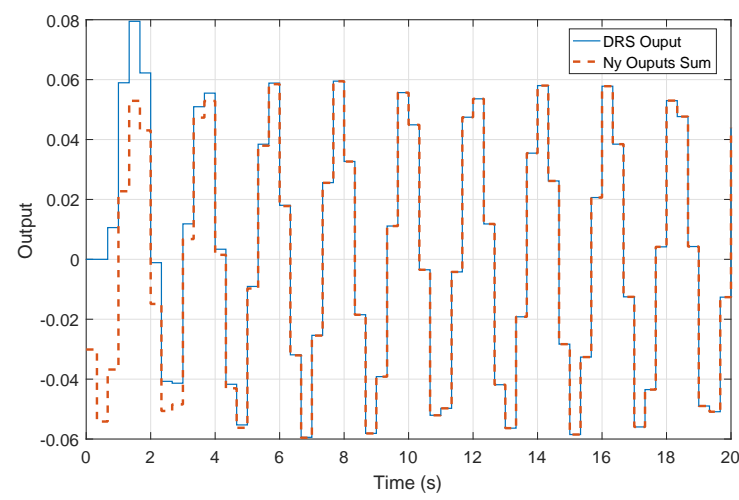

Figure 4.6: Output Components Sum Comparison
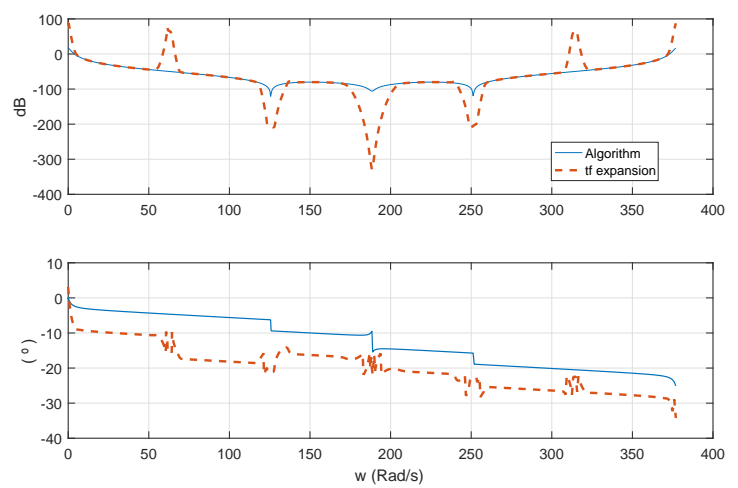

Figure 4.7: DRS Bode. Comparison of Methods

The mistake is even greater if the degree increases, considering for instance a metaperiod $T_{0}=0.1, T_{u}=T_{0} / 4$ and $T_{y}=T_{0} / 3$. Figure 4.8 plots this last case with unacceptable results.

However a problem is found if the purpose is to work with these diagrams in a classical way obtaining important values such as margins for stability. The reason is that this sum is not really a pure sine signal. It is possible to assume a shortcut, adding the different output components at a specific metaperiod. In the case that the multiplicities $N_{u}, N_{y}$ were coprime, the output is ${ }^{3}$

\footnotetext{
${ }^{3}$ Remember that in general there will be $\frac{N_{y}}{g c d\left(N_{u}, N_{y}\right)}$
} 

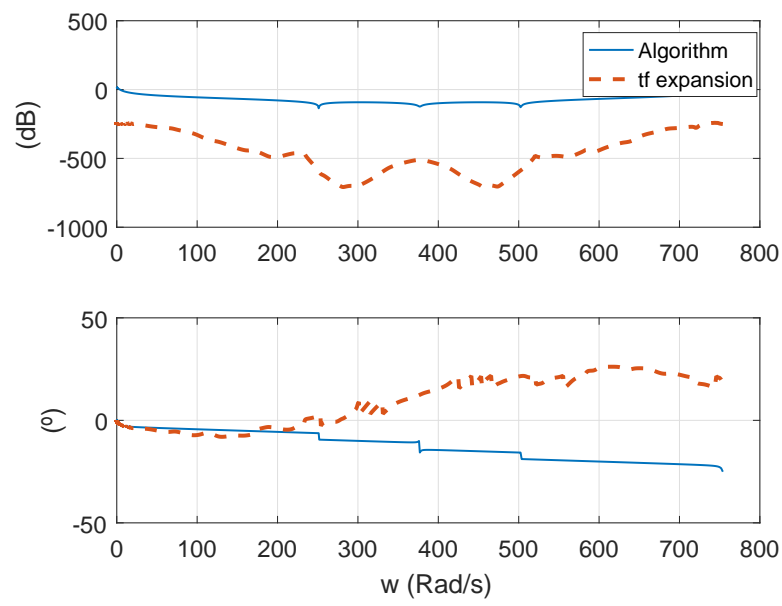

Figure 4.8: DRS Bode. Comparison of Methods

$$
\begin{aligned}
y\left(k T_{y}\right)= & A_{1} \sin \left(w k T_{y}+\varphi_{1}\right)+A_{2} \sin \left(\left(w+N_{u} w_{s}\right) k T_{y}+\varphi_{2}\right)+\cdots+ \\
& +A_{N_{y}} \sin \left(\left(w+\left(N_{y}-1\right) N_{u} w_{s}\right) k T_{y}+\varphi_{N_{y}}\right)
\end{aligned}
$$

As it was said this is not a pure sine signal. However if the decomposition of each component is observed

$$
\begin{aligned}
A_{\nu} \sin \left(w_{\nu} k T_{y}+\varphi_{\nu}\right)= & A_{\nu} \sin \left(w_{\nu} k T_{0}+\varphi_{\nu}\right)+ \\
& z_{T_{y}}^{-1} A_{\nu} \sin \left(w_{\nu}\left(k T_{0}+T_{y}\right)+\varphi_{\nu}\right)+\cdots \\
& z_{T_{y}}^{-\left(N_{y}-1\right)} A_{\nu} \sin \left(w_{\nu}\left(k T_{0}+\left(N_{y}-1\right) T_{y}\right)+\varphi_{\nu}\right)+ \\
& \text { for } \nu=1, \cdots,\left(N_{y}-1\right) \\
A_{\nu} \sin \left(w_{\nu} k T_{y}+\varphi_{\nu}\right)= & A_{\nu} \sin \left(w_{\nu} k T_{0}+\varphi_{\nu}\right)+ \\
& z_{T_{y}}^{-1} A_{\nu} \sin \left(w_{\nu} k T_{0}+\left(w_{\nu} T_{y}+\varphi_{\nu}\right)\right)+\cdots \\
& z_{T_{y}}^{-\left(N_{y}-1\right)} A_{\nu} \sin \left(w_{\nu} k T_{0}+\left(w_{\nu}\left(N_{y}-1\right) T_{y}+\varphi_{\nu}\right)\right)+ \\
& \text { for } \nu=1, \cdots,\left(N_{y}-1\right)
\end{aligned}
$$

So, adding the contributions of each component at $k T_{0}$, that is the skip of $y\left(k T_{y}\right)$ 


$$
\begin{aligned}
y^{T_{0}}\left(k T_{y}\right) & =A_{1} \sin \left(w k T_{0}+\varphi_{1}\right)+ \\
& +A_{2} \sin \left(w+w_{s}\right) k T_{0}+\left(\left(w+w_{s}\right) T_{y}+\varphi_{\nu}\right)+\cdots \\
& +A_{N_{y}} \sin \left(\left(\left(w+\left(N_{y}-1\right) w_{s}\right)+\left(N_{y}-1\right) N_{u} w_{s}\right) k T_{0}+\varphi_{N_{y}}\right)
\end{aligned}
$$

Finally leads to the $T_{0}$ approximation:

$$
\begin{aligned}
y^{T_{0}}\left(k T_{y}\right) & =A_{1} \sin \left(w k T_{0}+\varphi_{1}\right)+ \\
& +A_{2} \sin \left(w k T_{0}+\left(w_{s} T_{0}+\varphi_{\nu}\right)\right)+\cdots \\
& +A_{N_{y}} \sin \left(w k T_{0}+\left(N_{y}-1\right) w_{s} T_{0}+\varphi_{N_{y}}\right)
\end{aligned}
$$

The results of such approximation are depicted in Figure 4.9.

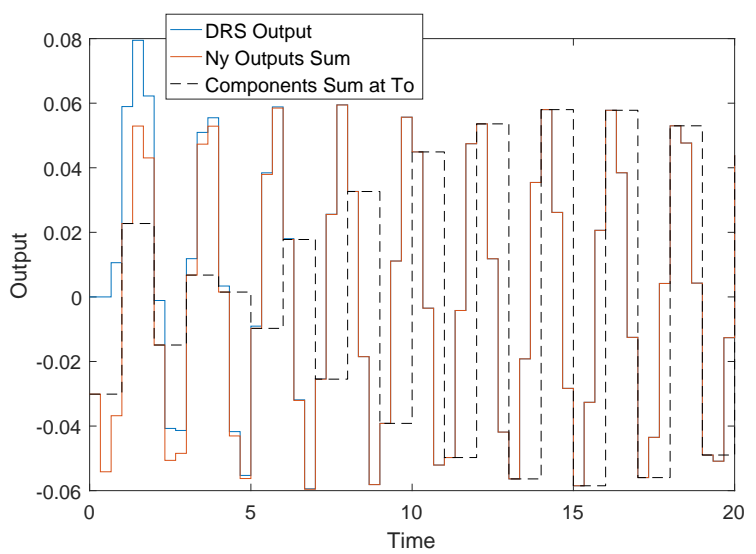

Figure 4.9: Output Components Sum at $T_{0}$ Comparison

These time responses have been achieved by using the diagram in Figure 4.10

\subsection{Stability Margins for DRS}

Once the DRS frequency response is known and its interpretation has been explained, the important concept of Stability Margins must be presented. In general, it could be said that the gain margin is the range of a gain $\alpha \in \mathbb{R}$ that multiplying the open loop transfer function system preserves the stability. In a Bode diagram it is usual to find the frequency for a $-\pi R a d$ and read the gain magnitude for that frequency. The interval from that value to $0 d B$ is the gain 


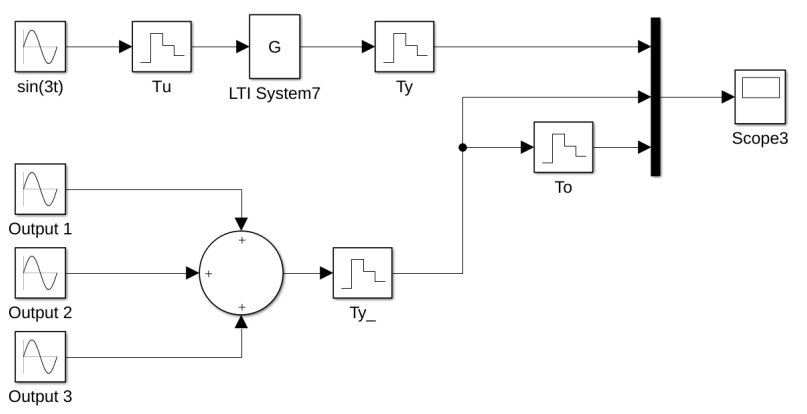

Figure 4.10: Simulation Diagram. Case $N_{y}=3$

margin, obviously if the value is under $0 d B$. Regarding the phase margin, now the frequency for which the $0 d B$ is read and the interval from the angle for that frequency to $-\pi$ Rad is this phase margin. In this case there is an interval as well but the minimum value is the minus positive angle and for this reason just the maximum value is given [20]. The same explanation could be applied for a DRS because it is possible to obtain a Bode diagram. As it was said before, this is just viable with the sum of all the output components for $T_{0}$ period (a metaperiod Bode). As it was explained it is an approximation, a very applicable one, as the practical experiences will show.

\subsubsection{Example}

Let us study the stability margins with the system

$$
G(s)=\frac{5}{s(s+1)(s+4)}
$$

with $T_{u}=T_{0} / 2$ and $T_{y}=T_{0} / 3$. The system is preceded by a ZOH. In this case, the $T_{0}=0.1$ metaperiod Bode diagram is depicted in Figure 4.11.

As it can be seen in Figure 4.11 a gain margin of $11.05 d B$ and a phase margin of $33.8^{\circ}$ are obtained. 

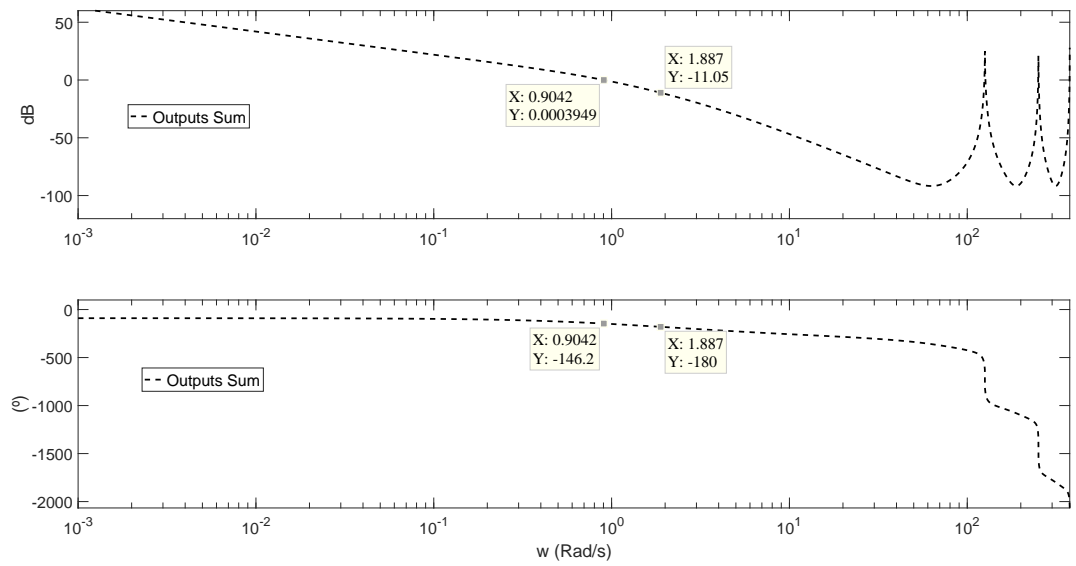

Figure 4.11: $T_{0}$ Bode Stability Margins Determination

The top signal in Figure 4.12 represents the simulation with a gain of $10^{\left(\frac{11.05}{20}\right)}$ and the bottom signal shows the time response when the system has a critical time delay of

$$
t_{\text {delay }}=\frac{(\text { Phase Margin })^{\circ}}{57.3 w_{0 d B}}=0.65 \mathrm{sec}
$$

Note that if the DRS is considered as a MIMO system and, as usual, the singular values of the MIMO transfer function frequency response are studied in this domain, it is more difficult to know the phase margin except when using a characteristic gain loci [20]

Figure 4.13 shows both the magnitude and the greatest singular value of the DRS frequency response in the metaperiod Bode diagram.

\subsection{Optimum $N$ Determination}

A question or a repetitive comment that every reader considers when she/he is reading a MRS contribution is: what is the best relation in a DRS when $T_{u}=T_{0} / N$ and $T_{y}=T_{0}$ ?, (that is in a MRIC scheme). Sometimes the $N$ is imposed by the specific problem but if the designer can choose the $N$ value, which will be the best one? Generally, at first, there is no way to determine an 

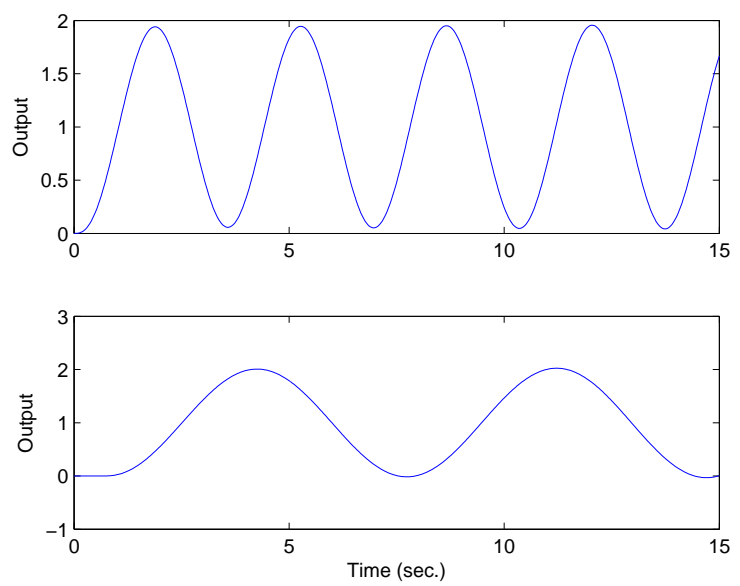

Figure 4.12: Simulation of DRS with Stability Margins consideration

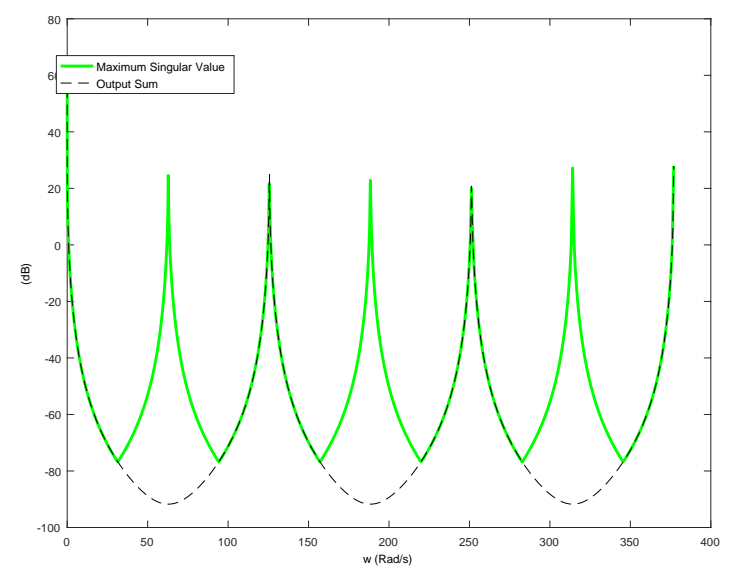

Figure 4.13: Metaperiod $T_{0}$ DRS Bode and the greatest Singular Value

optimum $N$ for all cases. But now, the designer has powerful tools. Studying a couple of examples, assuming different dual-rate controllers, some issues will be able to be extrapolated. 


\subsubsection{Example 1}

The following system is considered

$$
G(s)=\frac{1.5}{(s+0.5)(s+1.5)}
$$

controlled by a parallel structure PID

$$
G_{r}(s)=K_{p}\left(1+T_{d} s+\frac{1 / T_{i}}{s}\right)
$$

with $K_{p}=8, T_{d}=0.2$ and $T_{i}=8$ for desired specifications. Due to problems in measurement frequency, the sampling time at process output is restricted to $T_{0}=0.3 \mathrm{sec}$. If the control scheme (case $N=1$ ) is analysed it leads to an unacceptable behavior with excessive underdamping and slow response. The proposal is to apply the scheme in Figure 4.14. The non-conventional controller with slow input $T_{0}$ and fast output $T_{0} / N$ is composed by a digital hold, $H^{T_{0}, T_{0} / N}$, which repeats every $T_{0}$ input $N$ times, and the $T_{0} / N$ discretization $G_{R}^{T_{0} / N}$ of the analog controller $G_{r}(s)$ using a known discretization technique ${ }^{4}$. With these conditions, $e\left(k T_{0}\right)=e\left(k T_{0}+T_{0} / N\right)=\cdots=e\left(k T_{0}+(N-1) T_{0} / N\right)$ and using the procedure exposed in section 4.2 the lifting matrix can be obtained.

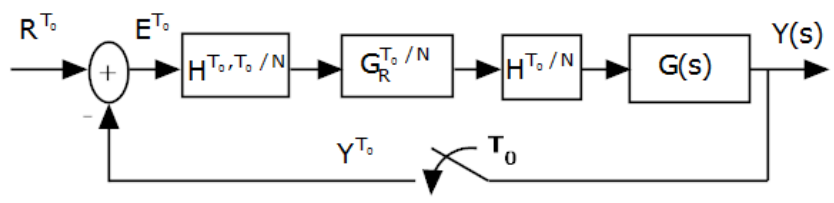

Figure 4.14: DR control scheme applied in example 1

Therefore, it is possible to obtain both the root locus and the Bode diagram ${ }^{5}$ regarding the $T_{0}$ open loop system for different values of $N=1,2,3,4, \ldots$. Figure 4.15, Figure 4.16 and Figure 4.17 depict the time response, the root locus and the Bode diagram respectively. From these figures it is easy to understand that increasing the value of $N$ a better performance is obtained, although the most intensive improvement is from $N=1$ to $N=2$. The

\footnotetext{
${ }^{4}$ In this case the formula exposed in [22]

${ }^{5}$ It is also possible to obtain the components Bode diagram if $G(s)$ fast intersampling is required. These diagrams must be done if ripple occurrence is suspected
} 
root locus is not able to clearly express the dynamic response because different closed loop poles and zeros prevent from an easy interpretation. The open loop Bode diagram is much clearer and it is observed how the margin phase is increasing with $N$ and therefore the underdamping is reduced. The gain margin is improved as well between $N=1$ and $N=2$, but among $N=2$ and successive values $N=3,4, \ldots$ the magnitude margin decreases. As it has been said, this is not a recipe for all processes and specifications but these comments are usually valid for a large variety of common processes. By obtaining the closed loop model and using the aforementioned tool, the frequency response of the closed loop $Y / R$ was obtained as well (see Figure 4.18). As it can be seen, the underdamping of the case $N=1$ is explained and it is deduced that the dual-rate cases present less high frequency noise rejection capability.

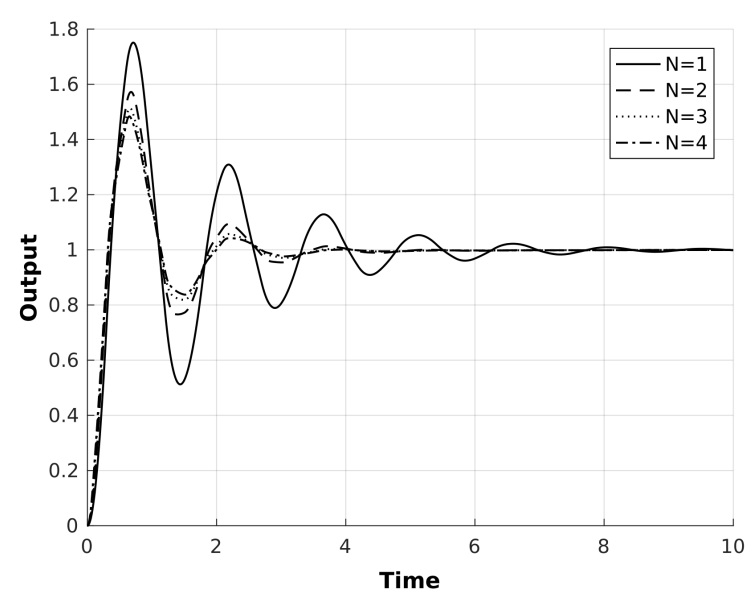

Figure 4.15: Time Response for different values of $N$

\subsubsection{Example 2}

Some works from author et al, [23, 24], introduced a special structure for a Dual-Rate Control scheme in a Multi rate input problem (that is more frequent input than output of the process). The detailed explanation can be read at [24, 25]. In [24] the time-design based procedure is exposed. Basically, this nonconventional structure dual-rate controller is composed by a slow part (working at the low frequency or measurement frequency) $G_{1}^{T_{0}}$, an expansion and digital holder $H^{T_{0}, T_{0} / N}$ to transform the slow signal output from slow dual-rate controller part, and a fast part (performing at updating control frequency or 


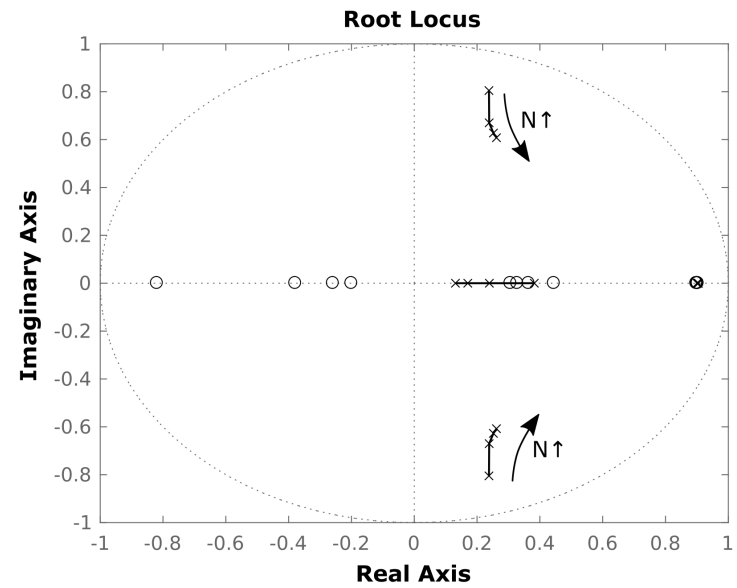

Figure 4.16: Root Locus for different values of $N$
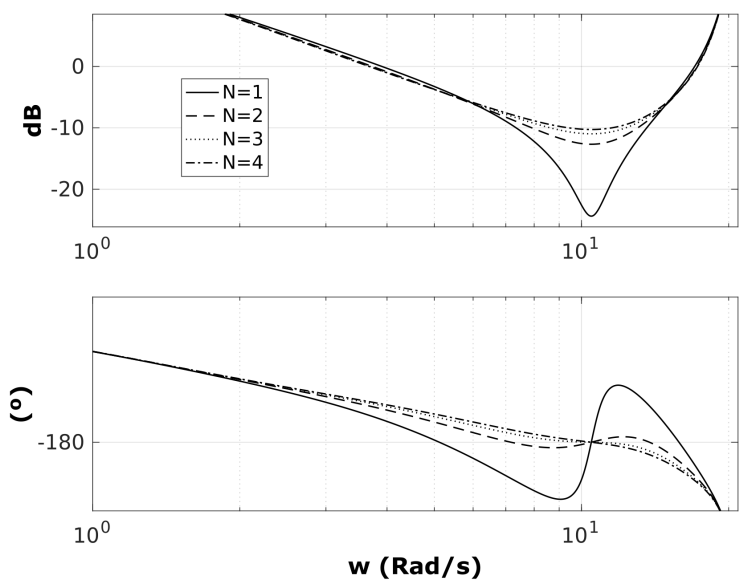

Figure 4.17: Open Loop Bode diagrams for different values of $N$

high frequency) $G_{2}^{T_{0} / N}$. In that contribution a model based design was introduced. The objective was to achieve a behavior settled by continuous closed loop transfer function $M(s)$. In addition the ideal closed loop response follows the $M(s) \mathrm{ZOH}$ discretization at fast and slow frequencies. Some problems with ripple could occur but there are ways to avoid it as is pointed out in the cited contribution. The non conventional dual-rate controller parts are 

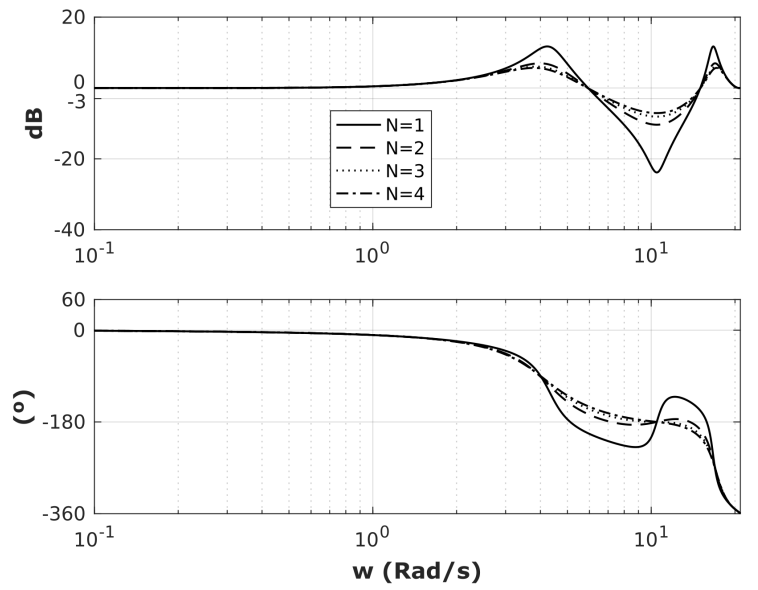

Figure 4.18: Closed Loop Bode diagrams for different values of $N$

$$
\begin{gathered}
G_{1}^{T_{0}}\left(z^{N}\right)=\frac{1}{1-M^{T_{0}}\left(z^{N}\right)} \\
G_{2}^{T_{0} / N}(z)=M^{T_{0} / N}(z) / G_{p}^{T_{0} / N}(z)=\frac{G_{R}^{T_{0} / N}(z)}{1+G_{R}^{T_{0} / N}(z) G_{p}^{T_{0} / N}(z)} \\
H^{T_{0}, T_{0} / N}(z)=\frac{R^{T_{0} / N}(z)}{\left(R^{T_{0}}\right)^{T}(z)}=\frac{1-z^{-N}}{1-z^{-1}}
\end{gathered}
$$

In Figure 4.19 the configuration is depicted. Note that the algebra introduced in section 4.2 was considered.

There are different ways to design this DR controller. From the easiest one, that consists in assuming $G_{1}^{T_{0}}=1$ (like in example 1) and preserves the fast controller $G_{R}^{T_{0} / N}(z)$ for $G_{2}^{T_{0} / N}$, with unpredictable behavior to more elaborated design procedures (see for instance [25]). In this contribution, the MR modelbased controller will be assumed [24] (Figure 4.19).

Now, a specific example in this case is introduced. With the same plant and PID as presented in example 1, the continuous closed loop transfer function is reached 


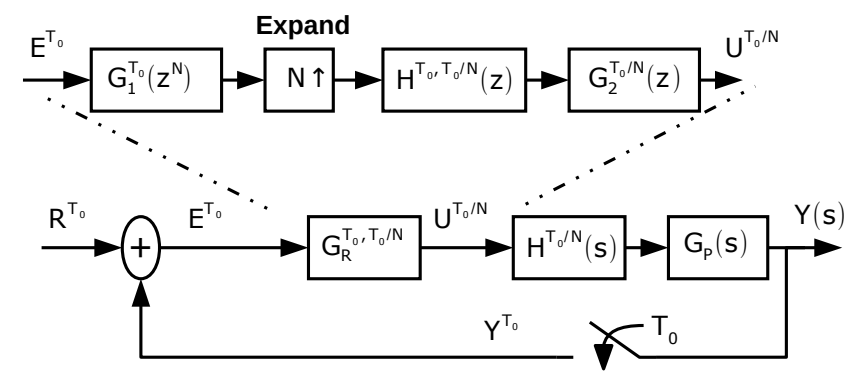

Figure 4.19: Closed Loop Dual-Rate Control. Non-conventional Dual-Rate Controller Structure used in example 2

$$
M(s)=\frac{2.4(s+4.66)(s+0.335)}{(s+0.33)\left(s^{2}+4 s+11.4\right)}
$$

With the same frame period, that is $T_{0}=0.3$ and successive values of $N=$ $2,3,4$ the non-conventional controllers parts are computed. Specifically, the results for $N=2$ are

$$
\begin{aligned}
G_{1}^{T_{0}}\left(z^{2}\right) & =\frac{z^{3}-1.656 z^{2}+0.0 .9741 z-0.2671}{z^{3}-2.328 z^{2}+1.72 z-0.3913} \\
G_{2}^{T_{0} / N}(z) & =\frac{23.55 z^{4}-74.51 z^{3}+86.81 z^{2}-43.92 z+8.08}{z^{4}-1.402 z^{3}-0.2546 z^{2}+1.141 z-0.4677}
\end{aligned}
$$

The application of single and dual-rate controllers for different $N$ values leads to the results shown in Figure 4.20, Figure 4.21, Figure 4.22 and Figure 4.23.

As it can be seen, some similar results are obtained, that is, there is an improvement in time response which is suspected from the Root Locus. The open loop Bode diagrams confirm this intuition adding that with this kind of controllers the best step is from $N=1$ to $N=2$. For successive values of $N$ a similar phase margin but lower gain margin is obtained. The sensitivity is worse when the value of $N$ is higher. 


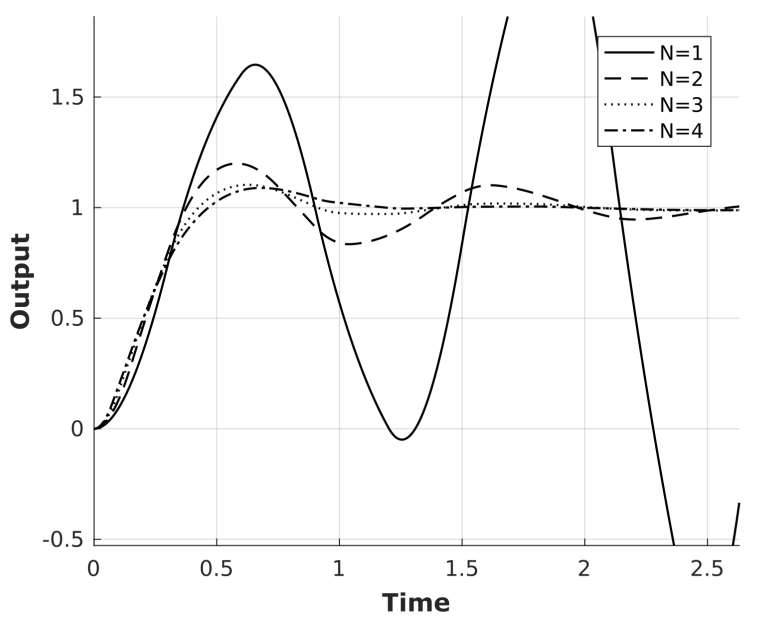

Figure 4.20: Time Response for different values of $N$

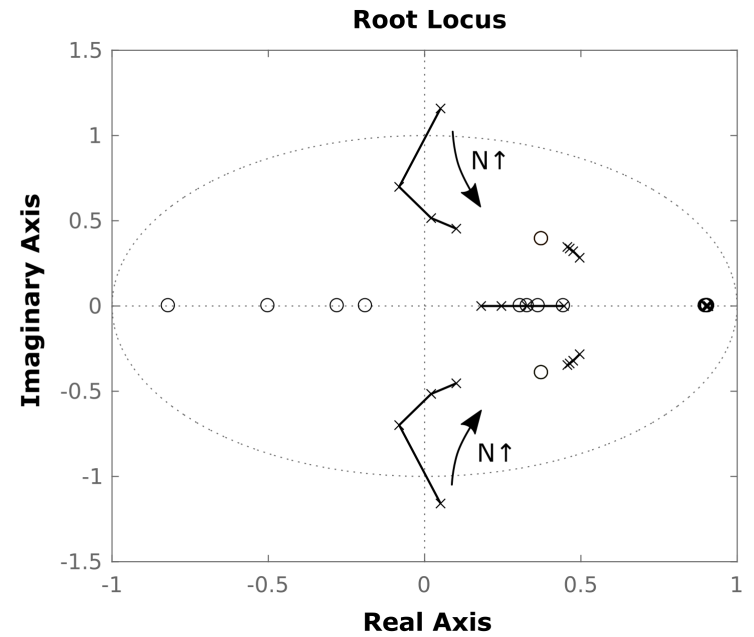

Figure 4.21: Root Locus for different values of $N$

\subsection{Conclusions}

In this contribution the interpretation of a new, proper and efficient way to obtain the frequency response of a DRS was introduced. It was also tested that 

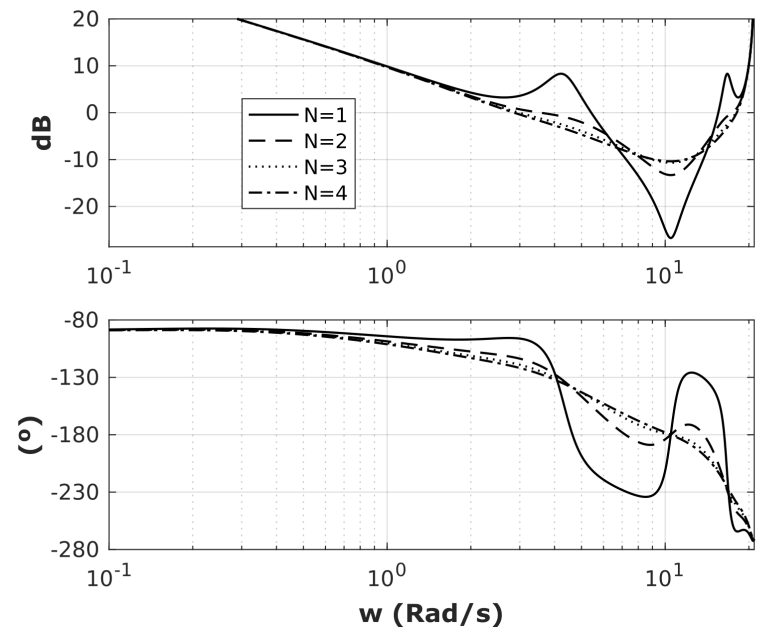

Figure 4.22: Open Loop Bode diagrams for different values of $N$

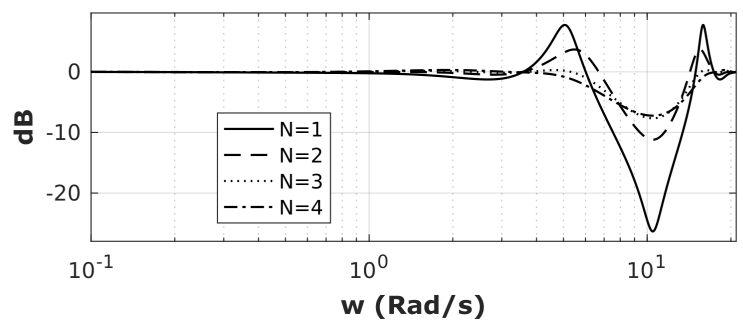

Figure 4.23: Closed Loop Bode diagrams for different values of $N$

the other methods for computing the MRS frequency response: MRS MIMO consideration and transfer function obtaining lead to partial or unacceptable results respectively. Some examples are introduced with the intention to clarify the understanding and consequences of this new method. This tool allows to properly ask some practical questions about MRS. 


\section{Disclosure statement}

No potential conflict of interest was reported by the authors.

\section{Notes on contributors}

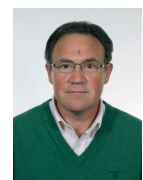

Julián Salt received the M.Sc. degree in industrial engineering and the Ph.D. degree in control engineering from the Technical University of Valencia, Valencia, Spain, in 1986 and 1992, respectively. He is a Full Professor with the Technical University of Valencia. He has been teaching during 32 academic years (from 1986) diverse contents in grade master and doctoral courses all related to Simulation, Automation and Systems Control. He has been advisor of different grade and master thesis and $7 \mathrm{PhD}$ in the last 10 years. All of these works were related to Non-Conventional Sampling Systems. He has co-authored over 70 papers in journals and conferences. His current research interests include nonconventionally sampled control and networked control systems.

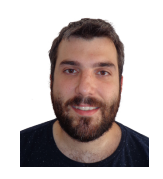

José Joaquin Alcaina received his M.Sc. degree in Automation and Industrial Computing from the Technical University of Valencia, Valencia, Spain, in 2015. He is currently completing his PhD studies in Automation, Robotics and Industrial Computer Science. His current research interests include networked control systems, packet-based dual-rate control and systems with low sensing ability. 



\section{References}

[1] G. Kranc. "Input-output analysis of multirate feedback systems". In: Automatic Control, IRE Transactions on 3.1 (1957), pp. 21-28 (cit. on p. 88).

[2] B. Friedland. Sampled Data Control Systems Containing Periodically Varying Numbers. United States Air Force, Office of Scientific Research, 1959 (cit. on p. 88).

[3] Thomas C Coffey and Ivan J Williams. "Stability analysis of multiloop, multirate sampled systems." In: AIAA Journal 4.12 (1966), pp. 21782190 (cit. on p. 88).

[4] Rudolf Emil Kalman and JE Bertram. "General synthesis procedure for computer control of single-loop and multiloop linear systems (An optimal sampling system)". In: Transactions of the American Institute of Electrical Engineers, Part II: Applications and Industry 77.6 (1959), pp. 602609 (cit. on p. 88).

[5] Ángel Cuenca et al. "A Delay-Dependent Dual-Rate PID Controller Over an Ethernet Network". In: IEEE Transactions on Industrial Informatics 7.1 (Feb. 2011), pp. 18-29. ISSN: 1551-3203. DOI: 10.1109/TII . 2010. 2085007 (cit. on p. 88).

[6] Nan Hou et al. "Event-triggered state estimation for time-delayed complex networks with gain variations based on partial nodes". In: International Journal of General Systems 47.5 (2018), pp. 477-490. DOI: 10. $1080 / 03081079$. 2018 .1462352. eprint: https : //doi .org/10.1080/ 03081079.2018.1462352 (cit. on p. 88).

[7] Xianye Bu et al. "Event-triggered distributed filtering over sensor networks with deception attacks and partial measurements". In: International Journal of General Systems 47.5 (2018), pp. 522-534. DOI: 10 . 1080/03081079 . 2018 . 1462353. eprint: https : //doi .org/10 . 1080/ 03081079.2018 .1462353 (cit. on p. 88). 
[8] Di Zhao et al. "PID output-feedback control under event-triggered protocol". In: International Journal of General Systems 47.5 (2018), pp. 432445. DOI: $10.1080 / 03081079.2018 .1459596$. eprint: https://doi.org/ 10.1080/03081079.2018.1459596 (cit. on p. 88).

[9] Dan Liu, Yurong Liu, and Fuad E. Alsaadi. "A new framework for output feedback controller design for a class of discrete-time stochastic nonlinear system with quantization and missing measurement". In: International Journal of General Systems 45.5 (2016), pp. 517-531. DOI: 10 . 1080/03081079 .2015 .1106737. eprint: https://doi .org/10.1080/ 03081079.2015 .1106737 (cit. on p. 88).

[10] Roberto Petrella and Marco Tursini. "An embedded system for position and speed measurement adopting incremental encoders". In: IEEE Transactions on industry applications 44.5 (2008), pp. 1436-1444 (cit. on p. 88).

[11] JSH Tsai, CM Chen, and LS Shieh. "Modelling of multirate feedback systems using uniform-rate models". In: Applied mathematical modelling 17.1 (1993), pp. 2-14 (cit. on p. 88).

[12] M. Cimino and P.R. Pagilla. "Design of linear time-invariant controllers for multirate systems". In: Automatica 46.8 (2010), pp. 1315-1319 (cit. on p. 88).

[13] M. Cimino and P.R. Pagilla. "Conditions for the ripple-free response of multirate systems using linear time-invariant controllers". In: Systems 8 Control Letters 59.8 (2010), pp. 510-516 (cit. on p. 88).

[14] Y.Y. Du et al. "Indirect identification of continuous-time delay systems from step responses". In: Applied Mathematical Modelling 35.2 (2011), pp. 594-611 (cit. on p. 88).

[15] B. Bamieh et al. "A lifting technique for linear periodic systems with applications to sampled-data control". In: Systems $\& 3$ Control Letters 17.2 (1991), pp. 79-88 (cit. on pp. 88, 90, 92).

[16] Feng Ding and Tongwen Chen. "Hierarchical identification of lifted statespace models for general dual-rate systems". In: IEEE Transactions on Circuits and Systems I: Regular Papers 52.6 (June 2005), pp. 1179-1187. ISSN: 1549-8328. DOI: 10.1109/TCSI . 2005.849144 (cit. on p. 88).

[17] P. Albertos. "Block multirate input-output model for sampled-data control systems". In: Automatic Control, IEEE Transactions on 35.9 (1990), pp. $1085-1088$ (cit. on pp. 88,92 ). 
[18] R. Meyer and C. Burrus. "A unified analysis of multirate and periodically time-varying digital filters". In: Circuits and Systems, IEEE Transactions on 22.3 (1975), pp. 162-168 (cit. on p. 88).

[19] J. Salt and A. Sala. "A new algorithm for dual-rate systems frequency response computation in discrete control systems". In: Applied Mathematical Modelling 38.23 (2014), pp. 5692-5704 (cit. on pp. 89, 92, 93).

[20] P. M. Thompson. "Gain and phase margins of multi-rate sampled-data feedback systems". In: International Journal of control 44.3 (1986), pp. 833846 (cit. on pp. 92, 99, 100).

[21] J. Salt, J. Torneno, and P. Albertos. "Modelling of non-conventional sampled data systems". In: Control Applications, 1993., Second IEEE Conference on (1993), pp. 631-635 (cit. on p. 92).

[22] R Iserman. Digital Control. 1990 (cit. on p. 102).

[23] Julián Salt and Pedro Albertos. "Multirate controllers design by rate decomposition". In: Proceedings of the 39th IEEE Conference on Decision and Control (Cat. No.00CH37187). Vol. 5. Dec. 2000, 4895-4900 vol.5. DOI: 10.1109/CDC.2001.914706 (cit. on p. 103).

[24] Julián Salt and Pedro Albertos. "Model-based multirate controllers design". In: IEEE Transactions on Control Systems Technology 13.6 (Nov. 2005), pp. 988-997. ISSN: 1063-6536. DOI: 10.1109/TCST . 2005.857410 (cit. on pp. 103, 105).

[25] Julián Salt et al. "A Multirate Control Strategy to the Slow Sensors Problem: An Interactive Simulation Tool for Controller Assisted Design". In: Sensors 14.3 (2014), pp. 4086-4110. ISSN: 1424-8220. DOI: 10.3390/ s140304086 (cit. on pp. 103, 105). 



\title{
Chapter 5
}

\section{Paper 4. Delay-independent dual-rate PID controller for a packet-based networked con- trol system}

\author{
José Alcaina, Ángel Cuenca, Julián Salt, Vicente \\ Casanova, Ricardo Pizá
}

In this paper, a novel delay-independent control structure for a networked control system (NCS) is proposed, where packet-based control strategies with predictor-based and dual-rate control techniques are integrated. The control solution is able to cope with some networked communication problems such as time-varying delays, packet dropouts and packet disorder. In addition, the proposed approach enables to reduce network load, and usage of connected devices, while maintaining a satisfactory control performance. As a delay-independent control solution, no network-induced delay measurement is needed for controller implementation. In addition, the control scheme is applicable to open-loop unstable plants. Control system stability is ensured in terms of linear matrix inequalities (LMIs). Simulation results show the main benefits of the control approach, which are experimentally validated by means of a Cartesian-robot-based test-bed platform.

Keywords: Networked control systems; multi-rate control; predictor-based control; packet-based control.

Information Sciences, volume 484, may 2019, pages 27-43, https: //doi.org/10.1016/j. ins.2019.01.059 


\subsection{Introduction}

Networked Control Systems (NCSs) [1, 2] is a prolific control area, addressing control scenarios where different devices share a common communication link. There are several advantages associated with NCS such as cost reduction, and ease of installation and maintenance, but also drawbacks like the possible occurrence of time-varying delays [3-11], packet dropouts [4, 7, 12-14], packet disorder [4, 7, 12, 15, 16], and network bandwidth constraints [17-19]. One interesting aim in NCS is to reduce the number of transmissions through the network, which can result in some advantages such as increase in network bandwidth, and enlargement of battery usage of the different wireless devices connected to the NCS. But the reduction in transmissions should come along with preservation of stability and performance properties. In order to ensure this achievement, different control solutions have been proposed in literature, for instance: packet-based control [20-22], which enables to decrease the communication rate by simultaneously sending a set of data in each transmission; event-based control [9, 23-27], where the transmission is only carried out if control or output variables satisfy a certain event condition; multi-rate control [28-30], where a slower sensing rate in comparison to a faster actuation can be assumed; and predictor-based control [26, 31-33], which exploits model-based predictions to address scarce data and compensate for network-induced delays. The present work proposes a control structure for an NCS, which integrates packet-based control strategies with predictor-based and dual-rate control techniques. The systematic combination of these control solutions enables not only to reduce the number of transmissions through the network but also to face some networked communication problems such as time-varying delays, packet dropouts, and packet disorder. In addition, the control approach is able to keep a satisfactory control performance, which is defined by means of the nominal (no-delay, no-dropout) dual-rate response.

Dual-rate control techniques provide twofold benefits: to avoid packet disorder and to reduce the number of transmissions through the network. The sensing period may be chosen to be larger than the largest round-trip time delay found in a statistical distribution for the network-induced delay, which is assumed to be known $[34,35]$. In this way, no packet disorder is produced, and the use of the network and devices can be reduced. Despite the fact of choosing a slow sensing period, a satisfactory control performance may be achieved by selecting a faster actuation period [30]. In the present work, due to the wide knowledge of PID controllers in industrial and academic environments, a dual-rate PID control structure is taken into account. The controller is split into two parts: a slow-rate PI controller and a fast-rate PD controller. The integral action 
is applied at slow rate because it usually operates at this frequency zone. As data travel through the network at slow rate, the PI controller is located at the remote side, with no direct connection to the plant. The derivative actions, which are associated with faster dynamics, are applied at fast rate to reach the satisfactory control performance, and hence, the PD controller is located at the local side, directly connected to the plant. Basic design procedures of dual-rate PID controllers can be looked up in [30].

Combining packet-based control strategies with predictor-based control techniques enables to deal with packet dropouts and time-varying delays. Packet dropouts may occur because of the utilization of user datagram protocol (UDP) as the communication protocol in this work $[34,35]$. Predictor-based control techniques can be used at the remote side in order to deal with up to $M$ possible consecutive packet dropouts, being $M$ an upper-bound which may be established from off-line experiences in the NCS. The $M$ future, estimated PI control actions will be sent to the local side by implementing a packet-based control strategy in order to compute the next PD control actions. This computation can be carried out following a delay-free control algorithm, which is a central aspect of this work. At the local side, when no packet arrives, the PD controller is able to compute an estimated control signal from the PI control actions received in the previous transmission, and then, it can apply the signal following a uniform actuation pattern, that is, not being influenced by the time-varying network-induced delay. When the packet arrives after the delay, the PD controller is able to compute the actual control actions irrespective of the delay, and replace the estimated control signal with the actual one. Assuming neither model uncertainties nor disturbances, the difference between actual and estimated control signals should be negligible. Note that, inside the current sensing period, the control signal estimated for this period is injected from the beginning of the period to the moment in which the new packet is received. This is an essential difference compared with [36], where the last control action of the previous sensing period is held along the current sensing period until the new packet arrives, being additionally required a gain scheduling approach to retune the controller according to the delay. To the best of the authors' knowledge, the working mode of the delay-independent control algorithm proposed in this work is novel in this kind of frameworks. Since the present work is able to consider unstable plants to be controlled, the state prediction solution will include a state resetting procedure [36-38].

In summary, the proposed delay-independent dual-rate PID controller may be defined as a PID controller, which is able to generate a fast-rate control signal from a slow-rate process output measurement, calculating the fast-rate con- 
trol signal by following a delay-free control algorithm, which means that the network-induced delay does not affect the computation. Therefore, the motivation of introducing this kind of controller is twofold: i) for implementation purposes, an obvious advantage derived from the delay-independent control solution is that the round-trip time delay is not required to be measured. This feature makes the solution applicable to a wide range of NCSs where the time delay is difficult to measure; and ii) no additional control techniques (e.g., gain-scheduling control [36, 39], optimal control [15], fuzzy control [8, 9], $H_{\infty}$ filtering techniques [7], etc.) may be required to compensate for the delay, which reduces the complexity of the control solution,

Although the proposed controller is delay-independent, the plant, due to the time-varying network delay, is subjected to some variations in the instants where the input commands are presented to the plant. Then, the plant model can vary from sensor period to sensor period. If that were not the case, the eigenvalues of the NCS closed-loop model would determine stability and performance, but eigenvalues are of limited usefulness in time-varying contexts, such as the networked one in this paper. This leads to represent the NCS closed-loop model as a Linear Time Varying (LTV) system, which depends on the time-varying delay, and whose stability can be ensured via Linear Matrix Inequalities (LMIs).

Summarizing, the main contribution of this paper is the development of a new and comprehensive approach, where dual-rate and predictor-based control techniques and packet-based control strategies are systematically brought together in an NCS in order to face some communication drawbacks and reduce resource utilization, while maintaining a satisfactory control performance. The central feature of the proposed control solution is its delay-free control signal computation. This is a distinguished improvement compared to other control solutions, where the network-induced delay must be compensated for, and hence, additional control techniques may be required.

The paper is structured in the following sections. In section 5.2, the problem scenario is formally introduced. In section 5.3, the control techniques used at the remote and local sides are presented. Control system stability is enunciated in terms of LMIs in section 5.4. Simulation results in section 5.5 illustrate the benefits of the proposed control strategy in an unstable open-loop plant; concretely, by controlling the position output of a Cartesian robot. In section 5.6, the previous results are experimentally validated in a test-bed platform based on the Cartesian robot and using UDP as the network protocol. Finally, conclusions close this contribution. 


\begin{tabular}{|c|c|}
\hline \multicolumn{2}{|l|}{ Nomenclature } \\
\hline$N, t, T, N T$ & $\begin{array}{l}\text { Multiplicity, and basic, actuation } \\
\text { and sensor periods }\end{array}$ \\
\hline$\tau_{k}^{l r}, \tau_{k}^{r l}, \tau_{k}$ & $\begin{array}{l}\text { Local-to-remote, remote-to-local, } \\
\text { and round-trip time network- } \\
\text { induced delay }\end{array}$ \\
\hline$d_{k}^{l r}, d_{k}^{r l}$ & $\begin{array}{l}\text { Local-to-remote and remote-to-local } \\
\text { packet dropout occurrence }\end{array}$ \\
\hline$y_{k}^{N T}, \hat{y}_{k}^{N T}, \bar{y}_{k}^{N T}$ & $\begin{array}{l}\text { Actual, estimated, and actual or es- } \\
\text { timated, system output (at } N T)\end{array}$ \\
\hline$r_{k}^{N T}$ & Reference signal (at $N T)$ \\
\hline$e_{k}^{N T}$ & Error signal (PI input) (at $N T$ ) \\
\hline$x_{k}^{N T}, \hat{x}_{k}^{N T}, \bar{x}_{k}^{N T}$ & $\begin{array}{l}\text { Actual, estimated, and actual or es- } \\
\text { timated, system state (at } N T)\end{array}$ \\
\hline$v_{k}^{N T}, \hat{v}_{k}^{N T}, \bar{v}_{k}^{N T}$ & $\begin{array}{l}\text { Actual, estimated, and actual or es- } \\
\text { timated, PI control action (at } N T)\end{array}$ \\
\hline$\tilde{v}_{k}^{T}$ & Expanded PI control action (at $T$ ) \\
\hline$v_{k}^{T}, \hat{v}_{k}^{T}, \bar{v}_{k}^{T}$ & $\begin{array}{l}\text { Actual, estimated, and actual or es- } \\
\text { timated, PI action converted at } T\end{array}$ \\
\hline$u_{k}^{T}, \hat{u}_{k}^{T}, \bar{u}_{k}^{T}$ & $\begin{array}{l}\text { Actual, estimated, and actual or es- } \\
\text { timated, PD control action }(\text { at } T)\end{array}$ \\
\hline$M$ & Upper bound for estimations \\
\hline$z$ & $t$-unit operator \\
\hline $\bar{z}=z^{L}, L=\frac{T}{t}$ & $T$-unit operator \\
\hline$z_{N}=\bar{z}^{N}=z^{N \cdot L}$ & $N T$-unit operator \\
\hline
\end{tabular}




\subsection{Problem description}

The proposed NCS is depicted in Figure 5.1, where the network is placed between the remote and local sides, and it introduces some communication problems such as time-varying delays, packet dropouts, and packet disorder. In the next subsections, these problems are formally described, and the control structure is presented in detail.

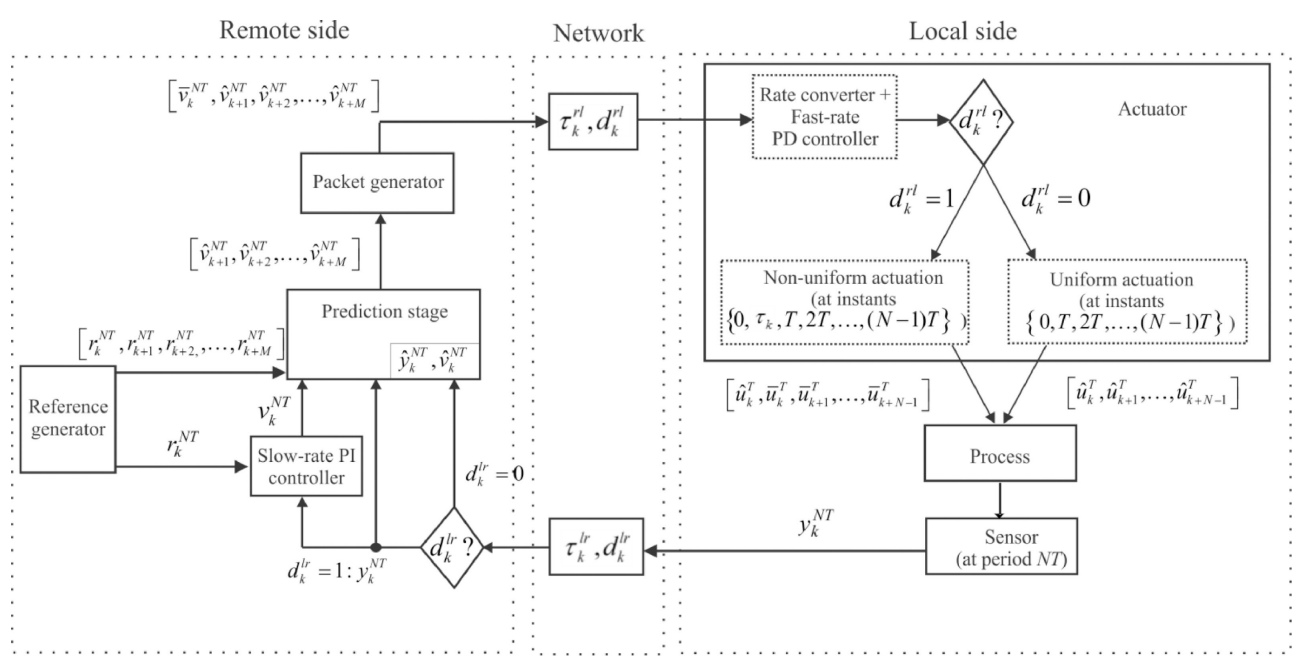

Figure 5.1: NCS scenario

\subsubsection{Time-varying delays, packet dropouts, and packet disorder}

The round-trip time delay for the packet sampled at instant $k N T$ (where $k \in \mathbb{N}$, $T$ is the actuation period, $N T$ is the sensor period, and $N \in \mathbb{Z}$ is a parameter known as multiplicity in a dual-rate control framework [30]) is defined as

$$
\tau_{k}=\tau_{k}^{l r}+\tau_{k}^{r l}+\tau_{k}^{c}
$$

being $\tau_{k}^{l r}$ the local-to-remote network-induced delay, $\tau_{k}^{r l}$ the remote-to-local delay, and $\tau_{k}^{c}$ a negligible computation time delay. In order to avoid packet disorder, in this work it is strictly necessary to know the maximum round-trip time delay $\tau_{\max }$ such that $\tau_{\max }<N T$. As a common timer is supposed to be shared by the local devices in such a way that all of them are perfectly synchronized, $\tau_{k}$ can be measured subtracting packet sending and receiving times, 
not requiring time-stamping techniques. Therefore, from off-line experiences in the NCS, the statistical distribution of the round-trip time delay can be obtained, and hence, $\tau_{\max }$. Since in this work, an IP network which uses UDP as the transport layer protocol is taken into account, the distribution will be a constant plus a Gamma distributed random variable, whose shape and scale parameters change with load and network segment [35]. Usually, this distribution is approximated as a generalized exponential distribution [39], whose probability density function can take this form

$$
P\left[\tau_{k}\right]= \begin{cases}\frac{1}{\phi} e^{\frac{-\left(\tau_{k}-\eta\right)}{\phi}} & , \tau_{k} \geq \eta \\ 0 & , \tau_{k}<\eta\end{cases}
$$

being the expected value of the delay $E\left[\tau_{k}\right]=\phi+\eta$ and its variance $V\left[\tau_{k}\right]=\phi^{2}$. A feasible choice of $\eta$ is the median of the delay. From $\eta$ and an experimental value of $E\left[\tau_{k}\right]$ (or the mean), $\phi$ can be easily approximated.

As well-known, when using the UDP transmission model, packet dropouts appear. This phenomenon is essentially random [34], and hence, it can be modeled as a Bernoulli distribution [1]. The variable $d_{k}^{l r}$ indicates the possible loss of the packet sent from the local side to the remote one at the instant $\mathrm{kNT}$. Similarly, $d_{k}^{r l}$ is defined for the opposite network link. In this work, both variables are considered as a Bernoulli process with probability of dropout:

$$
\begin{aligned}
& p^{l r}=\operatorname{Pr}\left[d_{k}^{l r}=0\right] \in[0,1) \\
& p^{r l}=\operatorname{Pr}\left[d_{k}^{r l}=0\right] \in[0,1)
\end{aligned}
$$

In some real scenarios, $p^{l r}, p^{r l}$ could be considered as the same value $p=p^{l r}=$ $p^{r l}$.

Finally, let us define $M$ as the upper bound of consecutive packet dropouts. From a significant number of off-line experiences on the considered NCS, where some probability of dropout for the Bernoulli process in (5.3) can be chosen, one can observe the resulting number of consecutive packet dropouts which is produced in each experience. The largest number of consecutive packet dropouts for the whole set of experiences can be determined as the worst case. Although this number might appear with low probability, in order to consider a conservative decision it may be assigned to $M$. 


\subsubsection{Control structure}

Next, the different devices included in Figure 5.1 for the NCS are presented:

- the process to be controlled is a Cartesian robot. More information about this process can be found in section 5.5 and section 5.6.

- the sensor works at period $N T$ to sample the process output $y_{k}^{N T}$, which, in this case, is the position of the robot. Sensing at this slow rate enables to reduce the number of transmissions, reducing network load and device usage.

- the slow-rate PI controller generates a PI control action $v_{k}^{N T}$ from the reference $r_{k}^{N T}$ and the output $y_{k}^{N T}$, as long as the output arrives to the remote side (when ) after $\tau_{k}^{l r}$. Otherwise (when $d_{k}^{l r}=0$ ), a previously estimated PI control action $\hat{v}_{k}^{N T}$ will be used. Let us consider a maximum waiting time $\tau_{\max }^{l r}$ to detect a packet dropout in this device. If $\tau_{\max }^{l r}$ expires and the packet does not arrive, it will be considered as a dropout. More information about the definition and operation mode of the slow-rate PI controller can be found in subsection 5.3.2.

- the prediction stage computes an array of $M$ estimated, future PI control actions $\left[\hat{v}_{k+1}^{N T}, \hat{v}_{k+2}^{N T}, \cdots, \hat{v}_{k+M}^{N T}\right]$ taking into account: i) the array of the actual and future references $\left[r_{k}^{N T}, r_{k+1}^{N T}, r_{k+2}^{N T}, \cdots, r_{k+M}^{N T}\right]$, ii) the actual PI control action $v_{k}^{N T}$ or its estimation $\hat{v}_{k}^{N T}$, iii) the actual process output $y_{k}^{N T}$ or its estimation $\hat{y}_{k}^{N T}$, and iv) the actual state $x_{k}^{N T}$ or its estimation $\hat{x}_{k}^{N T}$. For the sake of simplicity and brevity, both cases (the actual and estimated ones) will be contained under the notation $\bar{v}_{k}^{N T}, \bar{y}_{k}^{N T}, \bar{x}_{k}^{N T}$ in the sequel. The main goals of the prediction stage are: i) to face packet dropouts for both network links; ii) to provide estimated PI control actions in order to be suitably used at the local side to compute the PD control signal via a delay-independent control algorithm. For more information about how the prediction stage works, see subsection 5.3.5.

- the packet generator implements a packet-based control strategy, which creates the packet to be sent to the local side, containing the set of estimated PI control actions $\left[\bar{v}_{k}^{N T}, \hat{v}_{k+1}^{N T}, \hat{v}_{k+2}^{N T}, \cdots, \hat{v}_{k+M}^{N T}\right]$.

- the actuator may include a rate converter and a fast-rate PD controller: firstly, the rate converter converts the slow-rate PI control signal into a fast-rate one to be used by the fast-rate PD controller as an input (more details in subsection 5.3.3). Secondly, the controller generates the fast-rate PD control signal in order to achieve the desired performance, 
which is defined by the nominal (no-delay, no-dropout) dual-rate control strategy. According to $d_{k}^{r l}$, the actuator applies the PD control actions using a different actuation pattern:

a) if dropout occurs $\left(d_{k}^{r l}=0\right)$ : the actuator injects the control actions following a uniform pattern at time instants $\{0, T, 2 T, \cdots$, $(N-1) T\}$ inside the current sensor period NT (see Figure 5.2). In this case, the PD control signal is computed from an estimated PI control value received in a previous successful delivery. Then, an estimated PD control signal $\left[\hat{u}_{k}^{T}, \hat{u}_{k+1}^{T}, \cdots, \hat{u}_{k+N-1}^{T}\right]$ is generated.

b) if no dropout occurs $\left(d_{k}^{r l}=0\right)$ : the actuator injects the control actions following a non-uniform pattern. For a particular pattern where $\tau_{k}<T$, the actuation time instants would be $\left\{0, \tau_{k}, T, 2 T, \cdots\right.$ , $(N-1) T\}$ inside the current sensor period $N T$ (see Figure 5.3). In this case, the PD control signal would take this form $\left[\hat{u}_{k}^{T}, \bar{u}_{k}^{T}\right.$, $\left.\bar{u}_{k+1}^{T}, \cdots, \bar{u}_{k+N-1}^{T}\right]$, which would mean the injection of an estimated PD control action $\hat{u}_{k}^{T}$ at the beginning of the current sensor period, and $N$ actual or estimated PD control actions $\bar{u}_{k+1}^{T}, i=[0 . . N-1]$ at the rest of the time instants after $\tau_{k}$. This subset of $N$ control actions will be composed of actual values, when an actual PI control signal $v_{k}^{N T}$ is used to compute them. Otherwise, when an estimated PI control signal $\hat{v}_{k}^{N T}$ is used, the subset will be composed of estimated values. In any case, assuming neither model uncertainties nor disturbances, $\hat{u}_{k}^{T}$ should be very similar to $\bar{u}_{k}^{T}$.

Under both actuation patterns, the PD control actions can be computed irrespective of the delay, resulting in a delay-independent control solution. This important feature distinguishes the solution, since no delay measurement is needed to implement the controller. More information about the fast-rate PD controller can be found in subsection 5.3.4.

\subsection{Control design}

The control proposal will be formulated in the next subsections. Firstly, some preliminaries, which are needed for the design, step will be presented. Secondly, the dual-rate controller will be stated. As commented in section 5.2, the controller is composed of a slow-rate PI controller, a fast-rate PD controller, and between them, a rate converter. Subsections 5.3.2 to 5.3.4 will detail the design aspects for each part of the dual-rate controller, differentiating between 


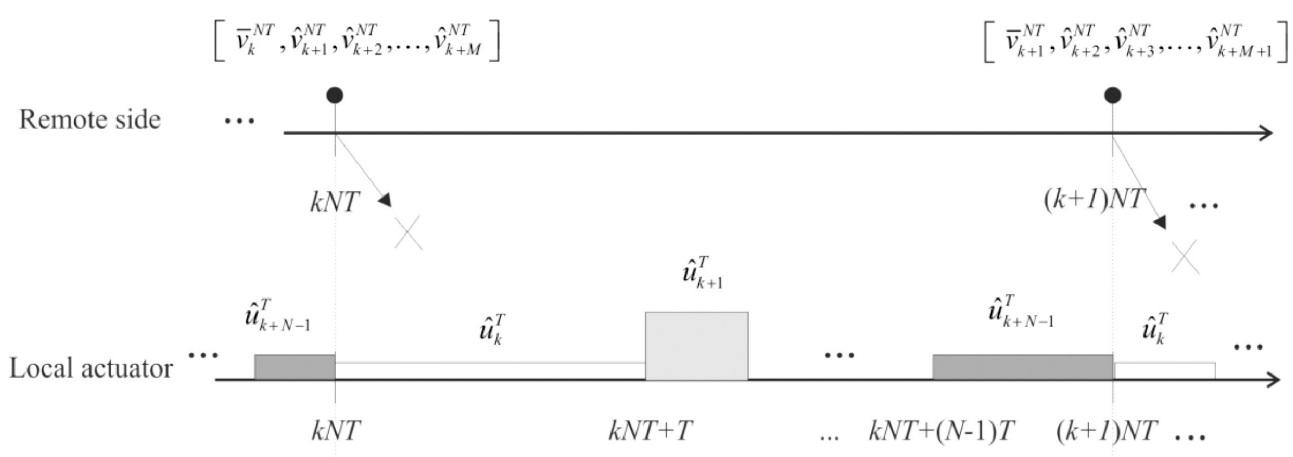

Figure 5.2: Packet dropout $\left(d_{k}^{r l}=0\right)$

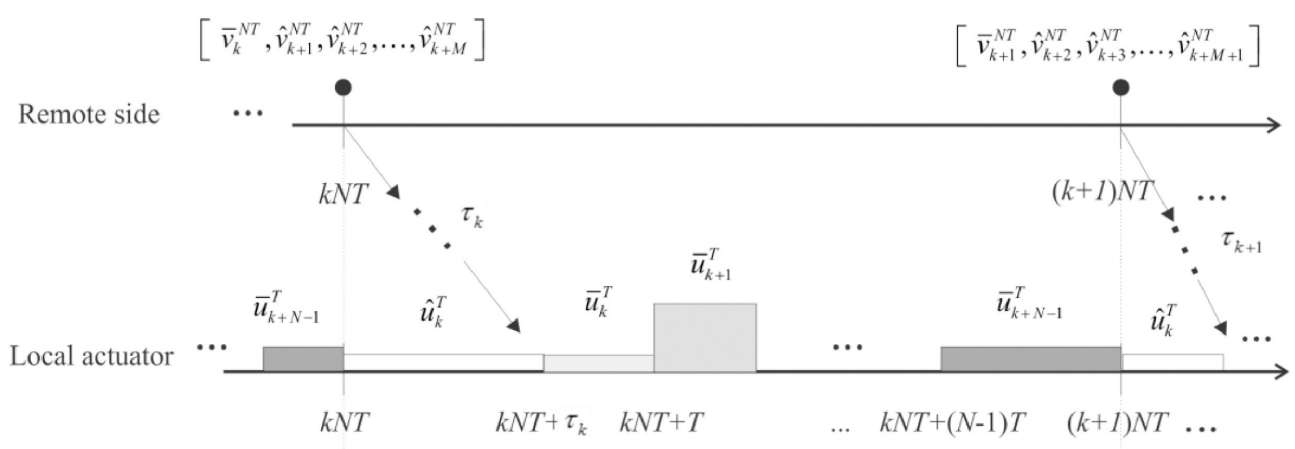

Figure 5.3: No packet dropout $\left(d_{k}^{r l}=1\right)$

the dropout case and the no-dropout case. Finally, in subsection 5.3.5, the prediction stage will be enunciated.

\subsubsection{Preliminaries}

Let us define the transfer function of the continuous plant to be controlled as $G_{p}(s)$. By using the Z-transform at different periods plus a zero-order hold device $H(s)$, different discrete-time versions for $G_{p}(s)$ can be considered. Therefore, denoting $\hat{z}=z^{L}$ and $z_{N}=\hat{z}^{N}=z^{N \cdot L}$ : 


$$
\begin{array}{r}
G^{N T}\left(z_{N}\right) \triangleq Z_{N T}\left[H_{N T} G_{p}(s)\right]=\frac{Y^{N T}\left(z_{N}\right)}{U^{N T}\left(z_{N}\right)} ; G^{T}(\bar{z}) \triangleq Z_{T}\left[H_{T} G_{p}(s)\right]=\frac{Y^{T}(\bar{z})}{U^{T}(\bar{z})} \\
G^{t}(z) \triangleq Z_{t}\left[H_{t} G_{p}(s)\right]=\frac{Y^{t}(z)}{U^{t}(z)}, \quad t<T: \quad t \cdot L=T, \quad L \in \mathbb{Z}^{+}
\end{array}
$$

In addition, the consequent state-space representations for each case (where matrices have suitable dimensions) can be enunciated as

$$
\begin{aligned}
& \begin{cases}x_{k+1}^{N T} & =A^{N T} x_{k}^{N T}+B^{N T} u_{k}^{N T} \\
y_{k}^{N T} & =C^{N T} x_{k}^{N T}\end{cases} \\
& \begin{cases}x_{k+1}^{T} & =A^{T} x_{k}^{T}+B^{T} u_{k}^{T} \\
y_{k}^{T} & =C^{T} x_{k}^{T}\end{cases} \\
& \begin{cases}x_{k+1}^{t} & =A^{t} x_{k}^{t}+B^{t} u_{k}^{t} \\
y_{k}^{t} & =C^{t} x_{k}^{t}\end{cases}
\end{aligned}
$$

Finally, let us consider a continuous PID controller, which is designed according to classical methods in order to achieve some specifications for the process to be controlled. The controller gains used for the next design steps are given by this PID configuration:

$$
G_{P I D}(s)=K_{p}\left(1+T_{d} s+\frac{1}{T_{i} s}\right)
$$

\subsubsection{Slow-rate PI controller}

a) No-dropout case $\left(d_{k}^{l r}=1\right)$ : The PI controller working at period $N T$ is enunciated as

$$
G_{P I}^{N T}\left(z_{N}\right)=K_{P I} \frac{z_{N}-\left(1-\frac{N T}{T_{i}}\right)}{z_{N}-1}=\frac{V^{N T}\left(z_{N}\right)}{E^{N T}\left(z_{N}\right)}
$$

being $V^{N T}\left(z_{N}\right)$ the PI control signal, $E^{N T}\left(z_{N}\right)$ the error signal, and $K_{P I}, T_{i}$ the gains of the PI controller. Let us consider $\left.K_{P I}=1\right)$. The PI control signal is obtained as 


$$
V^{N T}\left(z_{N}\right)=G_{P I}^{N T}\left(z_{N}\right) E^{N T}\left(z_{N}\right)=G_{P I}^{N T}\left(z_{N}\right)\left(R^{N T}\left(z_{N}\right) Y^{N T}\left(z_{N}\right)\right)
$$

and, from (5.7), the difference equation for the PI controller with $K_{P I}=1$ will be

$$
\begin{aligned}
v_{k}^{N T} & =v_{k-1}^{N T}+e_{k}^{N T}-\left(1-\frac{N T}{T_{i}}\right) e_{k-1}^{N T} \\
& =v_{k-1}^{N T}+\left(r_{k}^{N T}-y_{k}^{N T}\right)-\left(1-\frac{N T}{T_{i}}\right)\left(r_{k-1}^{N T}-y_{k-1}^{N T}\right)
\end{aligned}
$$

b) Dropout case $\left(d_{k}^{l r}=0\right)$ : In this case, instead of using the actual PI control signal in (5.8), the estimated one $\hat{V}_{N T}\left(z_{N}\right)$ must be used. This signal is previously generated at the prediction stage according to subsection 5.3.5.

\subsubsection{Rate converter}

As it is well-known [30], a rate converter $\left[H^{N T}\right]^{T}(\bar{z})$ is required between slow and fast rate controllers. Its goal is to convert the slow-rate PI control signal into a fast-rate one to be used by the fast-rate PD controller as an input. This operation, with low computational complexity, can be carried out at the local side. Two cases are considered:

a) No-dropout case $\left(d_{k}^{l r}=1\right)$ : The rate converter considers the actual slowrate PI control signal $V^{N T}\left(z_{N}\right)$ to obtain the held fast-rate one $V^{T}(\bar{z})$. As, in this work, step references are considered, the rate converter becomes a digital zero order hold:

$$
\begin{array}{r}
{\left[H^{N T}\right]^{T}(\bar{z})=\frac{V^{T}(\bar{z})}{\left[V^{N T}\left(z_{N}\right)\right]^{T}}=\frac{1-\bar{z}^{-N}}{1-\bar{z}^{-1}} \rightarrow} \\
V^{T}(\bar{z})=\left[H^{N T}\right]^{T}(\bar{z})\left[V^{N T}\left(z_{N}\right)\right]^{T}
\end{array}
$$

Note that $V^{N T}\left(z_{N}\right)$ is required to be used in an expanded way $\left[V^{N T}\left(z_{N}\right)\right]^{T}$, that is, 


$$
\begin{aligned}
{\left[V^{N T}\right]^{T}\left(z_{N}\right) } & \triangleq \tilde{V}^{T}(\bar{z}) \\
& \triangleq \sum_{k=0}^{\infty} \tilde{v}_{k}^{T} \bar{z}^{-k}:\left\{\begin{array}{ll}
\tilde{v}_{k}^{T}=v_{k}^{T}, & \forall k=\lambda N \\
\tilde{v}_{k}^{T}=0, & \forall k \neq \lambda N
\end{array}, \lambda \in \mathbb{Z}^{+}\right.
\end{aligned}
$$

More information can be found in [30]

b) Dropout case $\left(d_{k}^{l r}=0\right)$ : Now, the rate converter considers the estimated PI control signal $\hat{V}^{N T}\left(z_{N}\right)$

$$
\hat{V}^{T}(\bar{z})=\left[H^{N T}\right]^{T}(\bar{z})\left[\hat{V}^{N T}\left(z_{N}\right)\right]^{T}
$$

As said in section 5.2, for the sake of simplicity and brevity, both cases ((5.10) and (5.12)) will be contained under the notation $\bar{V}^{T}(\bar{z})$ from now on.

\subsubsection{Fast-rate PD controller}

a) No dropout $\left(d_{k}^{l r}=1\right)$ : Let us define $K_{P D}=K_{P}$ Then, the controller is stated as

$$
\begin{aligned}
G_{P D}^{T}(\bar{z}) & =K_{P D} \frac{\bar{z}\left(1+\frac{T_{d}}{T}\right)-\frac{T_{d}}{T}}{\bar{z}}=\frac{\bar{U}^{T}(\bar{z})}{\bar{V}^{T}(\bar{z})} \rightarrow \\
\bar{U}^{T}(\bar{z}) & =G_{P D}^{T}(\bar{z}) \bar{V}^{T}(\bar{z})
\end{aligned}
$$

and its difference equation is

$$
\bar{u}_{k}^{T}(\bar{z})=K_{P D}\left(1+\frac{T_{d}}{T}\right) \bar{v}_{k}^{T}-K_{P D}\left(\frac{T_{d}}{T}\right) \bar{v}_{k-1}^{T}
$$

Observe that the notation $\bar{U}^{T}(\bar{z})$ represents both $U^{T}(\bar{z})$ and $\hat{U}^{T}(\bar{z})$, where $U^{T}(\bar{z})$ is the actual PD control signal obtained from the actual PI control signal $V^{T}(\bar{z})$ (in (5.10), and $\hat{U}^{T}(\bar{z})$ is the estimated PD control signal obtained from the estimated PI control signal $\hat{U}^{T}(\bar{z})$ (in (5.12)). Iterating the difference equation deduced from (5.14) $N$ times, the $N$ PD control actions are generated and applied after $\tau_{k}$, which is the moment when $\bar{V}^{T}(\bar{z})$ is available. As commented in section 5.2, due to the delay, these actions will be applied following a non-uniform pattern. As there are 
different patterns depending on $\tau_{k}$, for the sake of clarity let us consider the particular case where $\tau_{k}<T$ (as in section 5.2). Then, a basic period $t$ is required to adapt the non-uniformity to the delay in such a way that the actuation pattern inside the sensor period $N T$ will take this form (where $l=0 . . L N-1$ ):

$$
\begin{cases}\hat{u}_{k}^{T}, & l t=0 . . \tau_{k} \\ \bar{u}_{k}^{T}, & l t=\tau_{k} . . T \\ \bar{u}_{k+1}^{T}, & l t=T . .2 T \\ \vdots & \\ \bar{u}_{k+N-1}^{T}, & l t=(N-1) T . . N T\end{cases}
$$

Note that the control action $\hat{u}_{k}^{T}$, which is injected at the beginning of the sensor period $N T$, is an estimated control action. Depending on the occurrence, or not, of a dropout in the local-to-remote link, the rest of control actions in (5.15), $\bar{u}_{k+i}^{T}, i=0 . . N-1$, will be estimated actions $\hat{u}_{k+i}^{T}$ or actual actions $u_{k+i}^{T}$, respectively. In the first case, $\bar{u}_{k}^{T}=\hat{u}_{k}^{T}$, and hence (5.15) is equivalent to (5.17). In the second case, $\bar{u}_{k}^{T}=u_{k}^{T}$, and assuming an accurate prediction, the difference between $\hat{u}_{k}^{T}$ and $u_{k}^{T}$ will be negligible.

b) Dropout case $\left(d_{k}^{l r}=0\right)$ : The estimated PI control signal $\hat{V}^{T}(\bar{z})$ (to be defined in the last step in subsection 5.3.5) is now required. This control signal is available at the local side, since it was calculated at the remote side in a previous successful communication.

The estimated PD control signal takes this form

$$
\hat{U}^{T}(\bar{z})=G_{P D}^{T}(\bar{z}) \hat{V}^{T}(\bar{z})
$$

From (5.14), but considering estimated signals, the set of $N$ control actions can be computed and applied according to the next uniform actuation pattern inside the sensor period $N T$ :

$$
\begin{cases}\hat{u}_{k}^{T}, & l t=0 . . T \\ \hat{u}_{k+1}^{T}, & l t=T . .2 T \\ \vdots & \\ \hat{u}_{k+N-1}^{T}, & l t=(N-1) T . . N T\end{cases}
$$




\subsubsection{Prediction Stage}

The prediction algorithm is executed $M$ times ( $M$ was defined in section 5.2 as the upper bound of consecutive packet dropouts) in order to generate the packet that includes the $M$ future, estimated PI control actions $\left[\hat{v}_{k+1}^{N T}, \hat{v}_{k+2}^{N T}\right.$, $\left.\cdots, \hat{v}_{k+M}^{N T}\right]$. This packet is computed for every sensor period at the remote side, and it is sent to the local side in order to be used when subsequent dropouts occur through the remote-to-local communication link. Considering a for-loop where $i=1 . . M$, the statements of the prediction algorithm included in the loop are based on the next steps:

a) Resetting of the initial state: If the current state sensed at period NT, $x_{k}^{N T}$, is available at the remote side, that is, no dropout occurs when being sent via the local-to-remote network link, a resetting of the initial condition for the state at period $t$ and $T$ can be carried out. This operation can be executed when $i=1$, and it is required to deal with possible unstable plants [36-38]. For the rest of iterations of the algorithm $(i=2 . . M)$, or if the current state was dropped when $i=1$, the updating is computed from the estimated state $\hat{x}_{k+i-1}^{N T}$ which will be defined in step 3 . As in section 5.2, to contemplate every situation, let us define a generic (actual or estimated) state $\dot{x}_{k}^{N T}$. Therefore, the resetting carried out in each iteration is

$$
\begin{cases}i=1: & \hat{x}_{k}^{T} \leftarrow \bar{x}_{k}^{N T} ; \quad \hat{x}_{k}^{t} \leftarrow \bar{x}_{k}^{N T} \\ i>1: & \hat{x}_{k+(i-1) N}^{T} \leftarrow \hat{x}_{k+i-1}^{N T} ; \quad \hat{x}_{k+(i-1) L N}^{t} \leftarrow \hat{x}_{k+i-1}^{N T}\end{cases}
$$

b) Estimation of the $N$ PD control actions either from the estimated PI control signal $\hat{V}^{T}(\bar{z})$ (this case can occur when $i \geq 1$ ) or from the actual one $V^{T}(\bar{z})$ (this case can occur only when $i=1$ ). Both cases assume the rate conversion previously carried out in ((5.12) or (5.10), respectively).

Similarly to (5.14), the estimated control signal is computed by iterating the next difference equation for $j=0 . . N-1$. Each iteration $i$ for the prediction algorithm is calculated as follows

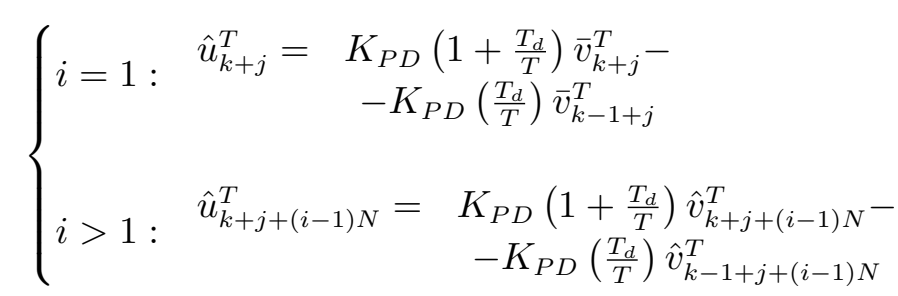


c) Estimation of the next state and output at period NT from the estimated PD control actions. Now, as in (5.17), a uniform pattern is used. Then, for each iteration of the prediction algorithm $i=1 . . M$, the next statespace representation at period $T$ is computed for $j=0 . . L N-1$

$$
\left\{\begin{array}{l}
\hat{x}_{k+1+j+(i-1) N}^{T}=A^{T} \hat{x}_{k+j+(i-1) N}^{T}+B^{T} \hat{u}_{k+j+(i-1) N}^{T} \\
\hat{y}_{k+1+j+(i-1) N}^{T}=C^{T} \hat{x}_{k+1+j+(i-1) N}^{T}
\end{array}\right.
$$

As a result of iterating (5.20) for all $i$, the $M$ estimated states and outputs at period $N T, \hat{x}_{k+i}^{N T}, \hat{y}_{k+i}^{N T}$, are calculated.

d) Estimation of the PI control signal $\hat{V}^{N T}\left(z_{N}\right)$ from the estimated output signal $\hat{Y}^{N T}\left(z_{N}\right)$. Note that, particularly for the first iteration of the prediction algorithm $(i=1)$, the actual output $y_{k}^{N T}$ can be used if it is available at the remote side, that is, if no dropout occurs when being sent through the local-to-remote network link $\left(d_{k}^{l r}=1\right)$. Then, the actual PI control action $v_{k}^{N T}$, which is generated by the output $y_{k}^{N T}$ (remember (5.9)), can also be used. In this way, similarly to step 1, a resetting of the initial condition for the PI controller $\left(v_{k}^{N T}\right)$ is carried out in order to compute the next estimated PI control action $\hat{v}_{k+1}^{N T}$. This operation is useful due to the unstable open-loop nature of the PI controller [3638]. As usual, in order to contemplate every situation in the prediction algorithm, let us define a generic (actual or estimated) output $\bar{y}_{k}^{N T}$, and a generic (actual or estimated) control action $\bar{v}_{k}^{N T}$. Therefore, similarly to (5.9), the different iterations $i$ of the prediction algorithm take the form

$$
\begin{cases}i=1: & \hat{v}_{k+1}^{N T}=\bar{v}_{k}^{N T}+\left(r_{k+1}^{N T}-\hat{y}_{k+1}^{N T}\right)-\left(1-\frac{N T}{T_{i}}\right)\left(r_{k}^{N T}-\bar{y}_{k}^{N T}\right) \\ i>1: & \hat{v}_{k+i}^{N T}=\hat{v}_{k+i-1}^{N T}+\left(r_{k+i}^{N T}-\hat{y}_{k+i}^{N T}\right)-\left(1-\frac{N T}{T_{i}}\right)\left(r_{k+i-1}^{N T}-\hat{y}_{k+i-1}^{N T}\right)\end{cases}
$$

\subsection{Stability analysis}

\subsubsection{Closed-loop model via lifting}

Let us consider a continuous linear time-invariant plant, which admits a statespace realization $\Sigma=(\bar{A}, \bar{B}, C, D)$, with suitable dimensions. Being $\xi$ an 
arbitrary real number, one can denote $B(\xi)=\int_{0}^{\xi} e^{\bar{A} \gamma} \bar{B} d \gamma$ if $\xi>0$, or $B(\xi)=0$ if $\xi \leq 0$. The discrete time sampled-data model at period $T$ of the previous plant was presented in (5.5), being $A^{T}=e^{\bar{A} T} ; \quad B^{T}=B(T) ; \quad C^{T}=C$.

In order to reflect the dual-rate sampling with either uniform actuation (when $d_{k}^{l r}=0$ ) or non-uniform actuation (when $d_{k}^{l r}=1$ ), the control system can be modeled via state-space representation adopting the so-called lifting methodology [40]. Then, let us represent the process as:

$$
\begin{aligned}
x_{k+1}^{N T} & =A_{p} x_{k+1}^{N T}+B_{p} \tilde{U}^{T} \\
y_{k}^{N T} & =C_{p} x_{k}^{N T}
\end{aligned}
$$

where

- $A_{p}=e^{\bar{A} N T} ; \quad B_{p}=\left[\begin{array}{lll}B_{0}^{*} & B_{1}^{*} \cdots B_{N}^{*}\end{array}\right] ; \quad C_{p}=C ;$ where $\bar{N}=N$ for the non-uniform case and $\bar{N}=N-1$ for the uniform case. In addition, $B_{i}^{*}=B\left(\lambda_{i+1}-\lambda_{i}\right) e^{\bar{A}\left(N T-\lambda_{i+1}\right)}$, where $\lambda_{i}(i=0,1, \cdots, \bar{N})$ are the actuation time instants.

- $\tilde{U}_{k}^{T}$ is a generic array of control signals to indistinctly represent either the uniform actuation as in (5.17) via $\hat{U}_{k}^{T}$, or the non-uniform actuation as in $(5.15)$ via $\hat{\bar{U}}_{k}^{T}$, where the particular case $\tau_{k}<T$ is considered.

The predictor stage can be modelled as follows:

$$
\hat{x}_{k+1}^{N T} \begin{cases}A_{p} \hat{x}_{k}^{N T}+B_{p} \tilde{U}_{k}^{T}, & \text { if } d_{k}^{l r}=0 \\ A_{p} x_{k}^{N T}+B_{p} \tilde{U}_{k}^{T}, & \text { if } d_{k}^{l r}=1\end{cases}
$$

For the sake of simplicity and brevity, let us consider the following assumptions to define the state-space representation of the delay-dependent controller:

1. the setpoints are constant, and hence we can assume them to be zero without loss of generality. Then, $e_{k}^{N T}=-y_{k}^{N T}$

2. the control system behavior when $d_{k}^{l r}=0$ is similar to the one when $d_{k}^{r l}=0$, since in both cases the control signal $\tilde{U}_{k}^{T}$ is computed from the estimated output $\hat{y}_{k}^{N T}$.

3. the dual-rate controller can be defined as a cascade-connected system. 
After manipulating the difference equations (5.9) and (5.14), and assuming that the integral actions are generated at slow rate, and the derivative actions are computed at fast rate (as commented in section 5.1), the dual-rate controller can take this lifted expression

$$
\begin{aligned}
& \left\{\begin{array}{l}
\varphi_{k+1}^{N T}=A_{c} \varphi_{k}^{N T}-B_{c} \hat{y}_{k+1}^{N T}+\bar{B}_{c} \hat{y}_{k}^{N T} \\
\hat{U}_{k}^{T}=C_{c} \varphi_{k}^{N T}
\end{array}, \text { if } d_{k}^{l r}=0 \text { or } d_{k}^{r l}=0\right. \\
& \left\{\begin{array}{l}
\varphi_{k+1}^{N T}=A_{c} \varphi_{k}^{N T}-B_{c} y_{k+1}^{N T}+\bar{B}_{c} y_{k}^{N T} \\
\hat{U}_{k}^{T}=\chi U_{k}^{T}+\left(\chi_{I}-\chi\right) \hat{U}_{k}^{T}=\bar{C}_{c} \varphi_{k}^{N T}
\end{array}, \quad \text { if } d_{k}^{l r}=d_{k}^{r l}=1\right.
\end{aligned}
$$

where, using $(\cdot)^{T}$ as transpose function, the controller state is $\varphi_{k}^{N T}=\left(\begin{array}{ll}v_{k}^{N T} & \mu_{k}^{N T}\end{array}\right)^{T}$, being $\mu_{k}^{N T}=v_{k-1}^{N T}$, and

$$
\begin{aligned}
A_{c} & =\left(\begin{array}{cc}
1 & 0 \\
1 & 0
\end{array}\right) ; \quad B_{c}=\left(\begin{array}{l}
1 \\
0
\end{array}\right) ; \quad \bar{B}_{c}=\left(\begin{array}{c}
1-\frac{N T}{T_{i}} \\
0
\end{array}\right) ; \\
C_{c} & =\left(\begin{array}{cc}
K_{P D}\left(1+\frac{T_{d}}{T}\right) & -K_{P D} \frac{T_{d}}{T} \\
K_{P D} & 0 \\
\vdots & \vdots \\
K_{P D} & 0
\end{array}\right)_{N \times 2}
\end{aligned}
$$

In addition, depending on the delay $\tau_{k}$, different configurations for $\chi, \chi_{I}$ and $\bar{C}_{c}$ may be considered. Therefore, when $\tau_{k}<T$

$$
\begin{aligned}
& \chi=\left(\begin{array}{c}
0_{1 \times N} \\
I_{N \times N}
\end{array}\right) ; \quad \chi_{I}=\left(\begin{array}{c}
I_{1 \times N} \\
I_{N \times N}
\end{array}\right) ; \\
& \bar{C}_{c}=\left(\begin{array}{cc}
K_{P D}\left(1+\frac{T_{d}}{T_{d}}\right) & -K_{P D} \frac{T_{d}}{T_{d}} \\
K_{P D}\left(1+\frac{T_{d}}{T}\right) & -K_{P D} \frac{\frac{T_{d}}{T}}{K_{P D}} \\
\vdots & 0 \\
K_{P D} & 0
\end{array}\right)_{(N+1) \times 2}
\end{aligned}
$$

when $\tau_{k}>d T, d=1 . . N-1$ 


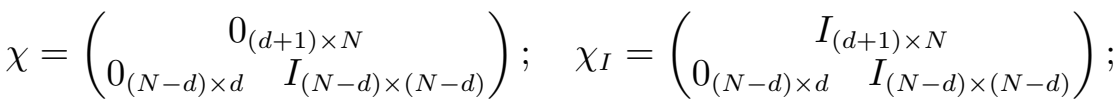

$$
\begin{aligned}
& \bar{C}_{c}=\left(\begin{array}{cc}
K_{P D}\left(1+\frac{T_{d}}{T}\right) & -K_{P D} \frac{T_{d}}{T} \\
K_{P D} & 0 \\
\vdots & \vdots \\
K_{P D} & 0
\end{array}\right)_{(N+1) \times 2}
\end{aligned}
$$

And finally, when $\tau_{k}=d T, d=1 . . N-1$

$$
\begin{aligned}
\chi & =\left(\begin{array}{cc}
0_{d \times N} \\
0_{(N-d) \times d} & I_{(N-d) \times(N-d)}
\end{array}\right) ; \quad \chi_{I}=\left(\begin{array}{cc}
I_{d \times N} \\
0_{(N-d) \times d} & I_{(N-d) \times(N-d)}
\end{array}\right) ; \\
\bar{C}_{c} & =\left(\begin{array}{cc}
K_{P D}\left(1+\frac{T_{d}}{T}\right) & -K_{P D} \frac{T_{d}}{T} \\
K_{P D} & 0 \\
\vdots & \vdots \\
K_{P D} & 0
\end{array}\right)_{N \times 2}
\end{aligned}
$$

Note that, as a delay-independent control solution, the controller in (5.24)(5.28) is defined irrespective of the delay $\tau_{k}$, that is, no controller's parameter is retuned according to the delay (as needed, for example, in [36]). In addition, when dropouts occur $\left(d_{k}^{l r}\right.$ or $d_{k}^{r l}$ ), as the configuration of $C_{c}$ does not depend on the delay because of the uniform actuation of the estimated control signal, the closed-loop model is also not dependent on the delay, $A_{c l, 0}$. However, when no packet dropout occurs $\left(d_{k}^{l r}=d_{k}^{r l}=1\right)$, the configuration of $\bar{C}_{c}$ does depend on the time-varying delay in order to satisfy the consequent input pattern to the plant. In this case, the plant is subjected to variations in the instants where the input commands are presented, and hence, matrices $A_{P}, B_{P}, C_{P}$ in (5.22) may vary from sensor period to sensor period. This leads to represent the NCS closed-loop model as a LTV system depending on $\tau_{k}, A_{c l, l}\left(\tau_{k}\right)$.

To obtain the closed-loop system, the following definition is used

$$
w_{k}^{N T}=y_{k}^{N T}-\hat{y}_{k}^{N T}=C_{p}\left(x_{k}^{N T}-\hat{x}_{k}^{N T}\right)
$$

and this state variable is adopted $\tilde{x}_{k}^{N T}=\left(\begin{array}{lll}x_{k}^{N T} & \varphi_{k}^{N T} & w_{k}^{N T}\end{array}\right)^{T}$. Some manipulations lead to the closed-loop system: 


$$
\tilde{x}_{k+1}^{N T}= \begin{cases}A_{c l, 0} \tilde{x}_{k}^{N T} & \text { if } d_{k}^{l r}=0 \text { or } d_{k}^{r l}=0 \\ A_{c l, 1}\left(\tau_{k}\right) \tilde{x}_{k}^{N T} & \text { if } d_{k}^{l r}=d_{k}^{r l}=1\end{cases}
$$

where

$$
\begin{gathered}
A_{c l, 0}=\left[\begin{array}{ccc}
A_{p} & B_{p} C_{c} & 0 \\
\bar{B}_{c} C_{p}-B_{c} C_{p} A_{p} & A_{c}-B_{c} C_{p} B_{p} C_{c} & -\bar{B}_{c}-A_{p} B_{c} \\
0 & 0 & A_{p}
\end{array}\right] \\
A_{c l, 1}\left(\tau_{k}\right)=\left[\begin{array}{ccc}
A_{p} & B_{p} \bar{C}_{c} & 0 \\
\bar{B}_{c} C_{p}-B_{c} C_{p} A_{p} & A_{c}-B_{c} C_{p} B_{p} \bar{C}_{c} & 0 \\
0 & 0 & 0
\end{array}\right]
\end{gathered}
$$

\subsubsection{Closed-loop stability}

To assess the closed-loop system stability, the next Theorem can be enunciated.

Theorem: Given $P\left[\tau_{k}\right]$ in (5.2), which is normalized to take values in $(0,1]$, the closed-loop system in (5.30)-(5.31) is stable if there exists a positive definitive solution $Q=Q^{T}>0$ for the following LMIs

$$
\begin{array}{r}
A_{c l, 0}^{T} \cdot Q \cdot A_{c l, 0}-Q<0 \\
\sum_{j=1}^{l} P\left[\vartheta_{j}\right] A_{c l, 1}^{T}\left(\vartheta_{j}\right) \cdot Q \cdot A_{c l, 1}\left(\vartheta_{j}\right)-Q<0
\end{array}
$$

where $\vartheta$ is a dummy parameter ranging in a set $\Theta$ where $\tau_{k}$ is assumed to take values in, being $\Theta$ an interval $\left[0, \tau_{\max }\right]$, and $l$ the number of equally spaced values to get a dense enough gridding in $\vartheta$. To solve (5.32), widely known methods [41] can be used.

Proof: Let $\tilde{V}_{k}^{N T}=\left(\bar{x}_{k}^{N T}\right)^{T} \cdot Q \cdot \bar{x}_{k}^{N T}$ be a Lyapunov candidate. Taking $E\left[\tilde{V}_{k}^{N T}\right]$ as the statistical expectation for the Lyapunov function, and assuming a probabilistic LMI gridding procedure, then the expectation of the increment $E\left[\Delta \tilde{V}_{k}^{N T}\right]$ along subsystem $A_{c l, 1}\left(\tau_{k}\right)$ in (5.30)-(5.31) can be obtained as follows 


$$
\begin{aligned}
E\left[\Delta \tilde{V}_{k}^{N T}\right] & =E\left[\tilde{V}_{k+1}^{N T}-\tilde{V}_{k}^{N T}\right]=E\left[\left(\tilde{x}_{k+1}^{N T}\right)^{T} \cdot Q \cdot \tilde{x}_{k+1}^{N T}-\left(\tilde{x}_{k}^{N T}\right)^{T} \cdot Q \cdot \tilde{x}_{k}^{N T}\right] \\
& =E\left[\left(A_{c l, 1}\left(\tau_{k}\right) \cdot \tilde{x}_{k}^{N T}\right)^{T} \cdot Q \cdot A_{c l, 1}\left(\tau_{k}\right) \cdot \tilde{x}_{k}^{N T}-\left(\tilde{x}_{k}^{N T}\right)^{T} \cdot Q \cdot \tilde{x}_{k}^{N T}\right] \\
& =E\left[\left(\tilde{x}_{k}^{N T}\right)^{T} A_{c l, 1}^{T}\left(\tau_{k}\right) \cdot Q \cdot A_{c l, 1}\left(\tau_{k}\right) \cdot \tilde{x}_{k}^{N T}-\left(\tilde{x}_{k}^{N T}\right)^{T} \cdot Q \cdot \tilde{x}_{k}^{N T}\right] \\
& =E\left[\left(\tilde{x}_{k}^{N T}\right)^{T}\left(A_{c l, 1}^{T}\left(\tau_{k}\right) \cdot Q \cdot A_{c l, 1}\left(\tau_{k}\right)-Q\right) \cdot \tilde{x}_{k}^{N T}\right] \\
& =\left(\tilde{x}_{k}^{N T}\right)^{T}\left(\sum_{j=1}^{l} P\left[\vartheta_{j}\right] A_{c l, 1}^{T}\left(\vartheta_{j}\right) \cdot Q \cdot A_{c l, 1}\left(\vartheta_{j}\right)-Q\right) \tilde{x}_{k}^{N T}<0
\end{aligned}
$$

A similar development for subsystem $A_{c l, 1}\left(\tau_{k}\right)$ leads to (5.31).

By including every possible delay in $\vartheta$, system stability can be ensured for the different configurations of the controller in (5.26)-(5.28). Regarding $A_{c l, 0}$, a similar development can be carried out, not considering neither the expectation nor the delay. Finally, both developments lead to (5.32).

Discussion on feasibility: Note that the first inequality in (5.32), i.e. $A_{c l, o}^{T}$. $Q \cdot A_{c l, o}-Q<0$, would never hold if the plant were open-loop unstable (as the one considered in next Sections). However, if no model uncertainties and an accurate prediction were assumed (i.e. $w_{k}^{N T}$ ), the designed controller would be able to stabilize the plant. Then, under these conditions, in order to assess feasibility for the LMIs in (5.32), $A_{c l, 0}$ may be replaced by

$$
A_{c l, 0}=\left[\begin{array}{cc}
A_{p} & B_{p} C_{c} \\
\bar{B}_{c} C_{p}-B_{c} C_{p} A_{p} & A_{c}-B_{c} C_{p} B_{p} C_{c}
\end{array}\right]
$$

And, $A_{c l, 1}\left(\tau_{k}\right)$ may consequently reduce its dimensions as follows

$$
A_{c l, 1}\left(\tau_{k}\right)=\left[\begin{array}{cc}
A_{p} & B_{p} \bar{C}_{c} \\
\bar{B}_{c} C_{p}-B_{c} C_{p} A_{p} & A_{c}-B_{c} C_{p} B_{p} \bar{C}_{c}
\end{array}\right]
$$




\subsection{Simulation results}

This section is split into three subsections. In subsection 5.5.1, the data used in the simulations will be presented. In addition, stability for the proposed NCS will be assessed by means of the LMIs stated in section 5.4. The aim of subsection 5.5.2 will be to reveal the benefits of the proposed control solution by comparison with a delay-dependent approach in [36]. The system responses are shown and analyzed via some cost indexes. Finally, in subsection 5.5.3, model mismatches are considered for the delay-independent control solution, and their consequent effects on the system response are depicted and analyzed by means of some cost indexes.

\subsubsection{Simulation data. Control system stability assessment}

The process to be controlled is a Cartesian robot manufactured by Inteco, specifically, the 3D CRANE module (see in Figure 5.4). The rail measures of this plant for each axis are: $\mathrm{X}=0.050 \mathrm{~m}, \mathrm{Y}=0.040 \mathrm{~m}, \mathrm{Z}=0.050 \mathrm{~m}$.

Focusing on the $\mathrm{X}$ axis, its identification leads to

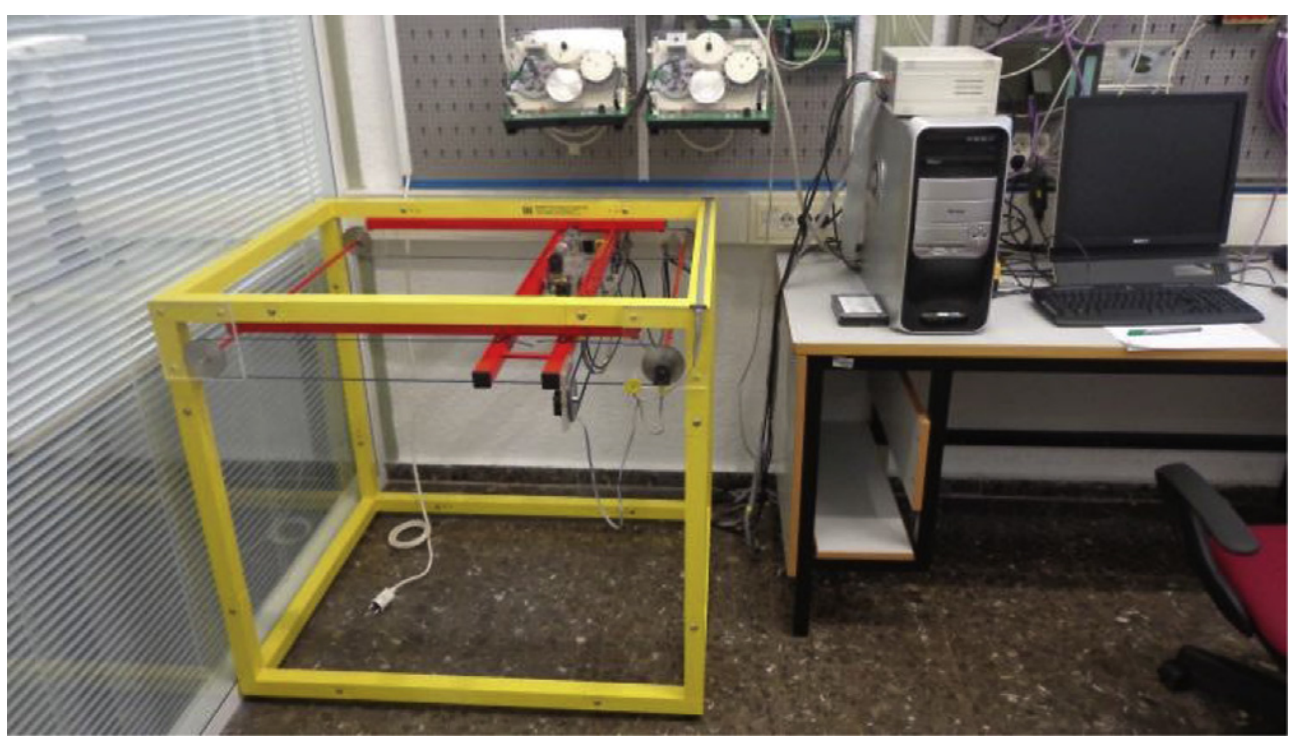

Figure 5.4: Cartesian robot (3D CRANE module)

$$
G_{p}(s)=\frac{6.3}{s(s+17.7)} m / c . a . u \text {. }
$$


where c.a.u. means control action units, which are generated by a PWM signal normalized in the range $[0,1]$.

The system also presents two non-linear behaviors to be taken into account in real-time implementation: saturation limits of control actions in \pm 1 , and dead zone values of \pm 0.06 . Both of them are identified experimentally and measured in normalized c.a.u.

For a fair comparison in the next subsection, the simulations are based on the consideration of a UDP network with the same parameters used in [36], that is,

- the network-induced delays are given by the histogram shown in Figure 5.5, which can be modeled as a generalized exponential distribution (5.2), where $\tau_{k}$ takes values in the range $\Theta=[0.04,0.08]$. As $\tau_{\max }=0.08$, then the sensor period can be chosen, for instance, as $N T=0.2 \mathrm{~s}$ in order to avoid packet disorder.

- the control design considers a conventional PID controller, being $K_{p}=12$, $T_{d}=0.01$ and $T_{i}=3.5$ in (5.6) in order to reach the following specifications: null steady-state error, settling time around $4 \mathrm{~s}$, and overshot around 5\%. From this controller, the dual-rate control is set up using (5.7)-(5.17), where $N=2$ is assumed.

- the packet dropouts are modeled by means of a Bernoulli distribution (2.3), being $p=p^{l r}=p^{r l}=0.3$ and $M=3$.

In order to assess the stability of the setup in probabilistic time-varying delays, the LMI gridding in (5.32) has been carried out taking $l=20$ grid points so as to compute the closed-loop realization $A_{c l, 1}\left(\vartheta_{j}\right)$ for the parameter space $\Theta$. From Figure 5.5, $P\left[\vartheta_{j}\right]$ is normalized in order to take values in $[0,1)$. As the plant in (5.36) is open-loop unstable, both $A_{c l, 0}$ and $A_{c l, 1}\left(\vartheta_{j}\right)$ in (5.34) and (5.35) are respectively used to check LMI feasibility. As a result, stability for the proposed NCS can be guaranteed, since the following feasible solution $Q$ exists

$$
Q=10^{2}\left[\begin{array}{rrrr}
0.311893 & 0.056373 & -0.076933 & -0.063061 \\
0.056373 & 0.661763 & 0.347536 & 0.023331 \\
-0.076933 & 0.347536 & 1.247753 & -0.032175 \\
-0.063061 & 0.023331 & -0.032175 & 0.329738
\end{array}\right]
$$




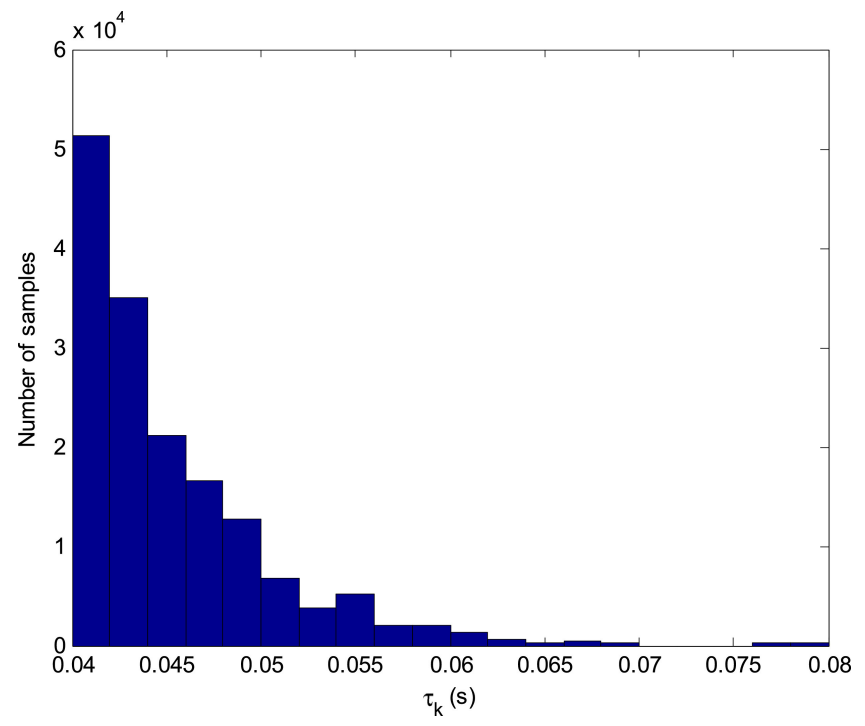

Figure 5.5: Delay histogram

\subsubsection{System responses. Cost indexes $J_{1}$ and $J_{2}$}

In this subsection, the delay-independent control solution proposed in this work will be compared to the delay-dependent approach based on a gain-scheduling technique presented in [36].

Figure 5.6 shows this comparison, where filtered step references (in dashed line) are used in order to avoid the saturation of the control signal. Note that the sequence of packet dropouts is represented in the time axis in such a way that each point indicates a packet dropout in the time instant where it is plotted. If the point increases its value in the vertical axis, then consecutive dropouts are occurring in this instant. Circles are used to show the delay-independent control solution, and a thin line is used to depict the delay-dependent approach. In addition, two more responses are included in Figure 5.6: the nominal, desired response, that is, the output obtained for the dual-rate control when no delay and no dropout are considered (in bold line), and the response for the dual-rate control when time-varying delays and dropouts occur but no prediction stage is included (in dotted line).

The simulation results show the next conclusions: i) as expected, the dual-rate control solution with no prediction stage is negatively affected by delays and dropouts, exhibiting the worst behavior, ii) as the delay-dependent approach 


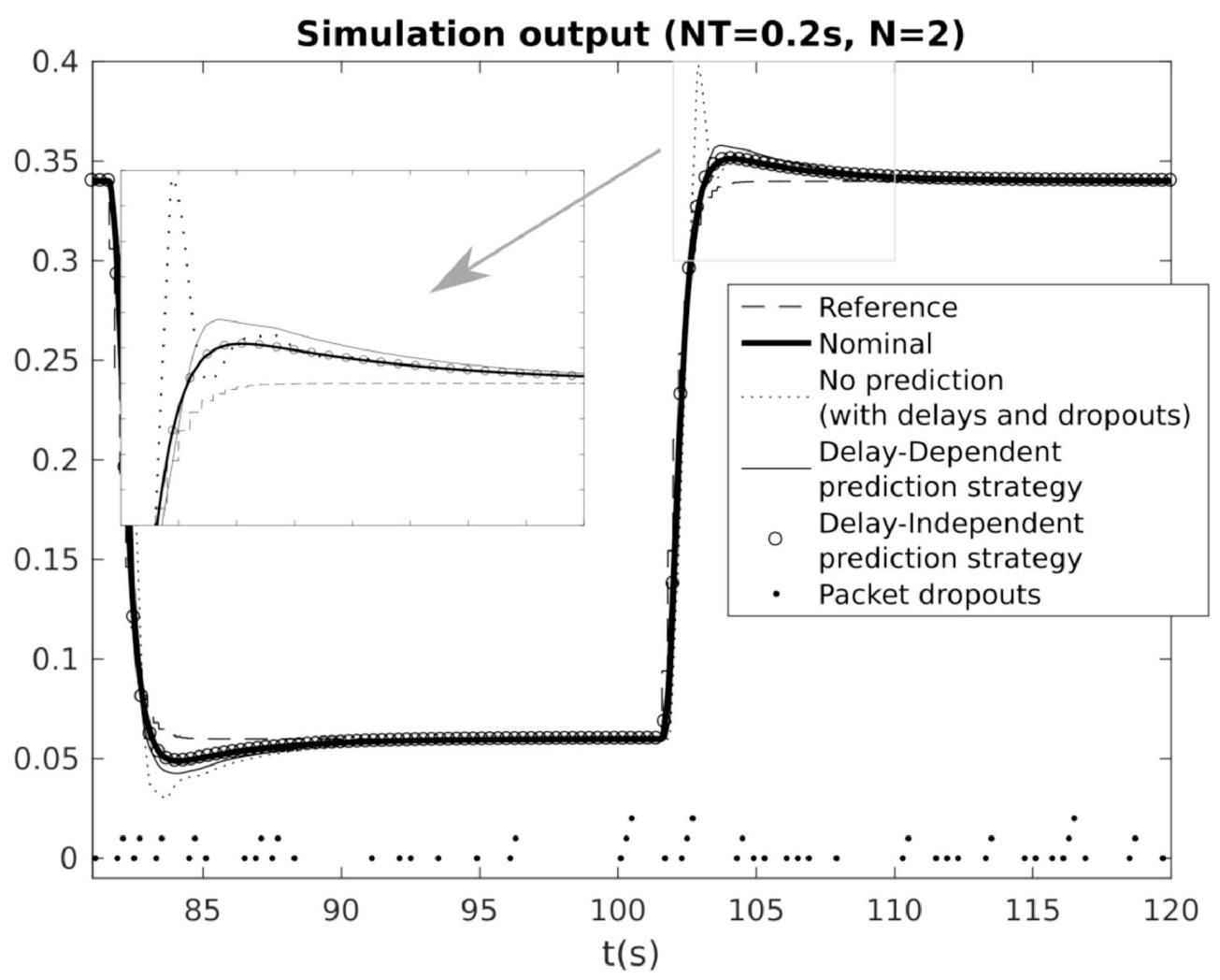

Figure 5.6: Comparison: nominal vs no prediction vs delay-dependent vs delay-independent

includes a prediction stage, it is able to restore the control performance, but it is not able to accurately reach the desired specifications; iii) the delayindependent control solution is able to achieve the nominal, desired behavior.

In order to better quantify these results, the cost indexes $J_{1}$ and $J_{2}$ are stated. $J_{1}$ is based on the Integral of Absolute Error (IAE), and $J_{2}$ on the overshoot value.

Let us consider the array $Y$, which includes the four control responses shown in Figure 5.6, that is, $Y=\left[Y_{N o m}, Y_{N P}, Y_{D D-P}, Y_{D I-P}\right]$, being $Y(1)=Y_{N o m}$ the output for the nominal (no-delay, no-dropout) dual-rate control, $Y(2)=Y_{N P}$ the output for the dual-rate control with no prediction stage and occurring delays and dropouts, $Y(3)=Y_{D D-P}$ the output for the delay-dependent approach (which includes prediction stage), and $Y(4)=Y_{D I-P}$ the output for 
the delay-independent proposal (which also includes prediction stage). From $Y$, the following accumulated (integrated) error $E_{Y}$ in a range of time instants $\Gamma$ can be computed

$$
E_{Y}(i)=\sum_{\Gamma}\left|Y(i)-Y_{\text {Nom }}\right|, \quad i=1 . .4
$$

Then, the $J_{1}$ cost index takes this form

$$
J_{1}(i)=100-\frac{E_{Y}(i)}{E_{Y}(2)} 100(\%), \quad i=1 . .4
$$

being $E_{Y}(2)$ the worst expected accumulated error, that is, the error calculated for $y(2)=Y_{N} P$. Therefore, the rest of the errors are measured by $J_{1}$ as an improvement (in \%) with regard to $E_{Y}(2)$.

Additionally, from $Y$ the following overshoot $O_{Y}$ in a range of time instants $\Gamma$ can be calculated (considering positive - $\max$ - or negative -min- filtered step references)

$$
O_{Y}(i)=\max \left(\left|\max _{\Gamma} Y(i)-\max _{\Gamma} Y_{N o m}\right|,\left|\min _{\Gamma} Y(i)-\min _{\Gamma} Y_{N o m}\right|\right), \quad i=1 . .4
$$

Then, the $J_{2}$ index is defined as

$$
J_{2}(i)=100-\frac{O_{Y}(i)}{O_{Y}(2)} 100(\%), \quad i=1 . .4
$$

being $O_{Y}(2)$ the worst expected overshoot, that is, the overshoot obtained for $Y(2)=Y_{N P}$. Similarly to $J_{1}$, the rest of the overshoots are measured by $J 2$ as an improvement (in \%) with regard to $O_{Y}(2)$.

Table 5.1 summarizes summarizes the cost indexes $J_{1}$ and $J_{2}$ obtained for each output. The delay-dependent control solution significantly improves $J_{2}$ (around 85\%) with respect to the worst response $Y_{N P}$, but it exhibits a poor improvement (around $42 \%$ ) for the value $J_{1}$. Nevertheless, the delay-independent control approach is able to accurately achieve the same control properties as the nominal dual-rate control solution, since $J_{1}$ and $J_{2}$ practically reach $100 \%$. 


\begin{tabular}{|l|llll|}
\hline Output & $E_{Y}$ & $J_{1}(\%)$ & $O_{Y}$ & $J_{2}(\%)$ \\
\hline$Y_{N P}$ & 291.19 & 0 & 0.047 & 0 \\
$Y_{D D-P}$ & 168.64 & 42.09 & 0.007 & 85.64 \\
$Y_{D I-P}$ & 0.02 & 99 & 0 & 100 \\
\hline
\end{tabular}

Table 5.1: Simulation: accumulated error $E_{Y}$ and cost index $J_{1}$; overshot $O_{Y}$ and cost index $J_{2}$

\subsubsection{Model mismatch. Cost indexes $J_{3}$ and $J_{4}$}

As the proposed delay-independent control solution is model-based, both the controller design and the prediction computation depend on how accurate the model represents the plant behavior. If some uncertainty between plant and model were assumed, the robustness of the model-based control proposal could be checked.

In this subsection, a certain model mismatch in the characteristic parameters of the plant, say, the static gain $K$ and the time constant $\tau$, will be considered. Let us consider a percentage $q \%$ of increase in $K, q \% \cdot \Delta K$, and a percentage $r \%$ of increase in $\tau, r \% \cdot \Delta \tau$. Figure 5.7 shows a comparison among the nominal response and the outputs obtained when $[20 \% \cdot \Delta K, 8 \% \cdot \Delta \tau]$ and $[30 \% \cdot \Delta K$, $12 \% \cdot \Delta \tau]$. As expected, the larger the percentage of uncertainty is considered, the worse the behavior becomes (with regard to the nominal response). Despite assuming a significant model mismatch when $[30 \% \cdot \Delta K, 12 \% \cdot \Delta \tau]$, the system remains stable. However, the response is clearly worsened, exhibiting $60 \%$ increase in settling time, and $6 \%$ increase in overshoot.

In order to better quantify these results and the robustness of the approach, the cost indexes $J_{3}$ and $J_{4}$ are stated. $J_{3}$ is based on the Integral of Absolute Error (IAE), and $J_{4}$ on the overshoot value.

Let us consider the matrix $W$, which includes different outputs for the proposed delay-independent approach. These outputs are obtained as a result of varying $q \% \Delta K$ and $r \% \Delta \tau$. In this study, $q$ takes the values $q=0,20,30$, and $r$ the values $r=0,8,12$. Combining these values, nine different responses in $W$ can be considered. The nominal response $Y_{\text {Nom }}$ is obtained when $q=r=0$. As previously commented, the worst behavior will be obtained for $q=30$ and $r=12$, since it represents the largest model mismatch. Let us assume this behavior as the worst permissible one. From $W$ the following accumulated (integrated) error $E_{W}$ in a range of time instants $\Gamma$ can be computed 


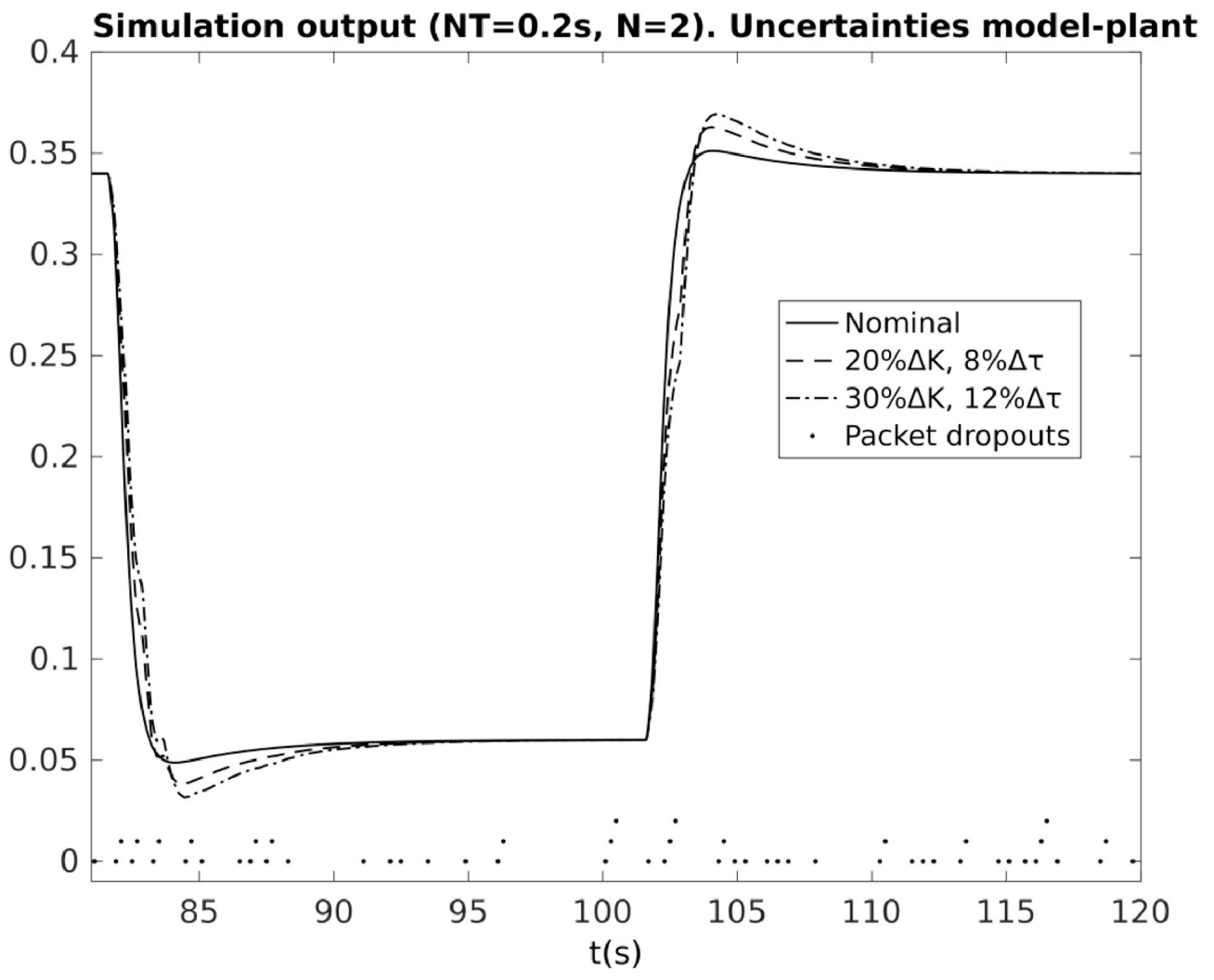

Figure 5.7: Comparison: nominal vs delay-independent (model mismatch)

$$
E_{W}\left(i_{r}, i_{q}\right)=\sum_{\Gamma}\left|W\left(i_{r}, i_{q}\right)-Y_{\text {Nom }}\right|, \quad i_{r}, i_{q}=1 . .3
$$

Then, the cost index takes this form

$$
J_{3}\left(i_{r}, i_{q}\right)=100-\frac{E_{W}\left(i_{r}, i_{q}\right)}{E_{W}(3,3)} 100(\%), \quad i_{r}, i_{q}=1 . .3
$$

being $E_{W}(3,3)$ the worst permissible accumulated error, that is, the error reached when $r=12$ and $q=30$. Therefore, the rest of the errors are measured by $J_{3}$ as an improvement (in \%) with regard to $E_{W}(3,3)$. 
5.5 Simulation results

\begin{tabular}{|c|c|c|c|c|c|c|c|c|c|}
\hline \multirow{2}{*}{\multicolumn{2}{|c|}{$E_{W}$}} & \multicolumn{3}{|c|}{$q \% \Delta K$} & \multirow{2}{*}{\multicolumn{2}{|c|}{$J_{3}$}} & \multicolumn{3}{|c|}{$q \% \Delta K$} \\
\hline & & 0 & 20 & 30 & & & 0 & 20 & 30 \\
\hline \multirow{3}{*}{$r \% \Delta \tau$} & 0 & 0 & 183.46 & 302.60 & \multirow{3}{*}{$r \% \Delta \tau$} & 0 & 100 & 54.94 & 25.68 \\
\hline & 8 & 59.82 & 248.62 & 373.11 & & 8 & 85.31 & 38.94 & 8.36 \\
\hline & 12 & 88.56 & 280.65 & 407.19 & & 12 & 78.25 & 31.07 & 0 \\
\hline
\end{tabular}

Table 5.2: Accumulated error $E_{W}$ and cost index $J_{3}$

\begin{tabular}{|c|c|c|c|c|c|c|c|c|c|}
\hline \multirow{2}{*}{\multicolumn{2}{|c|}{$O_{W}$}} & \multicolumn{3}{|l|}{$q \% \Delta K$} & \multirow{2}{*}{\multicolumn{2}{|c|}{$J_{4}$}} & \multicolumn{3}{|c|}{$q \% \Delta K$} \\
\hline & & 0 & 20 & 30 & & & 0 & 20 & 30 \\
\hline \multirow{3}{*}{$r \% \Delta \tau$} & 0 & 0 & 0.0087 & 0.0142 & \multirow{3}{*}{$r \% \Delta \tau$} & 0 & 100 & 52.19 & 21.97 \\
\hline & 8 & 0.0027 & 0.0117 & 0.0169 & & 8 & 85.16 & 35.71 & 7.14 \\
\hline & 12 & 0.0041 & 0.0130 & 0.0182 & & 12 & 77.47 & 28.57 & 0 \\
\hline
\end{tabular}

Table 5.3: Overshot $O_{W}$ and cost index $J_{4}$

Additionally, from $W$, the following overshoot $O_{W}$ in a range of time instants $\Gamma$ can be calculated (considering positive -max- or negative -min- filtered step references)

$$
\begin{aligned}
& O_{W}\left(i_{r}, i_{q}\right)= \\
& \max \left(\left|\max _{\Gamma} W\left(i_{r}, i_{q}\right)-\max _{\Gamma} Y_{\text {Nom }}\right|,\left|\min _{\Gamma} W\left(i_{r}, i_{q}\right)-\min _{\Gamma} Y_{\text {Nom }}\right|\right), \quad i=1 . .3
\end{aligned}
$$

And then, the $J_{4}$ cost index is defined as

$$
J_{4}\left(i_{r}, i_{q}\right)=100-\frac{O_{W}\left(i_{r}, i_{q}\right)}{O_{W}(3,3)} 100(\%), \quad i_{r}, i_{q}=1 . .3
$$

being $O_{W}(3,3)$ the worst permissible overshoot, that is, the overshoot reached when $r=12$ and $q=30$. Similarly to $J_{3}$, the rest of the overshoots are measured by $J_{4}$ as an improvement (in \%) with regard to $O_{W}(3,3)$.

Table 5.2 and Table 5.3 respectively summarize the cost indexes $J_{3}$ and $J_{4}$ obtained for each output depending on the model mismatch considered. As expected, the smaller the percentage of mismatch is considered, the larger $J_{3}$ and $J_{4}$ become, that is, a closer behavior to the nominal one is obtained. 


\begin{tabular}{|l|llll|}
\hline Output & $E_{Y}$ & $J_{1}(\%)$ & $O_{Y}$ & $J_{2}(\%)$ \\
\hline$Y_{N P}$ & 290.55 & 0 & 0.042 & 0 \\
$Y_{D D-P}$ & 207.53 & 28.58 & 0.012 & 70.80 \\
$Y_{D I-P}$ & 19.53 & 93.28 & 0.001 & 97.62 \\
\hline
\end{tabular}

Table 5.4: Experiment: accumulated error $E_{Y}$ and cost index $J_{1}$; overshot $O_{Y}$ and cost index $J_{2}$

\subsection{Experimental results}

To validate the simulation results obtained in section 5.5, a laboratory test-bed platform is set up, which includes the CRANE module previously presented, two computers and an Ethernet cable.

One computer is directly connected to the plant and includes the local part of the control system. The aims of this computer are: firstly, to be in charge of the sampling measurement and transmission at $N T=0.2 \mathrm{~s}$; secondly, to receive the packet which includes the current and predicted PI control signals; thirdly, to compute, and inject to the plant, the fast-rate PD control actions at $T=0.1 s$.

The second computer performs the remote part of the controller, receiving the outputs of the plant, calculating the current and the predicted slow-rate PI control actions, and sending back these actions to the local system. Both computers are connected by a UDP network through an Ethernet cable that performs the local-to-remote and remote-to-local links. In order to obtain the same conditions as those considered in simulation, packet delays and packet dropouts are modified by software.

Figure 5.8 shows the outputs obtained in the experiment, which clearly validates the trend observed in Figure 5.6, that is, the delay-independent control solution improves the results obtained by the delay-dependent approach in [36], being able to reach the nominal, desired behavior. To better validate the results, Table 5.4 details the cost indexes $J_{1}$ and $J_{2}$ computed for the experiment. While the delay-independent approach exhibits values for $J_{1}$ and $J_{2}$ very close to $100 \%$, which means that it practically achieves the nominal behavior, the delay-dependent proposal presents percentages which are significantly smaller.

Finally, to strengthen the previous conclusions, Figure 5.9 compares the behavior achieved by every control solution when the robot tries to track a $2 \mathrm{D}$ trajectory based on the well-known Lissajous curves (see, e.g., [42]). As expected, the response for the dual-rate control system when no prediction stage 


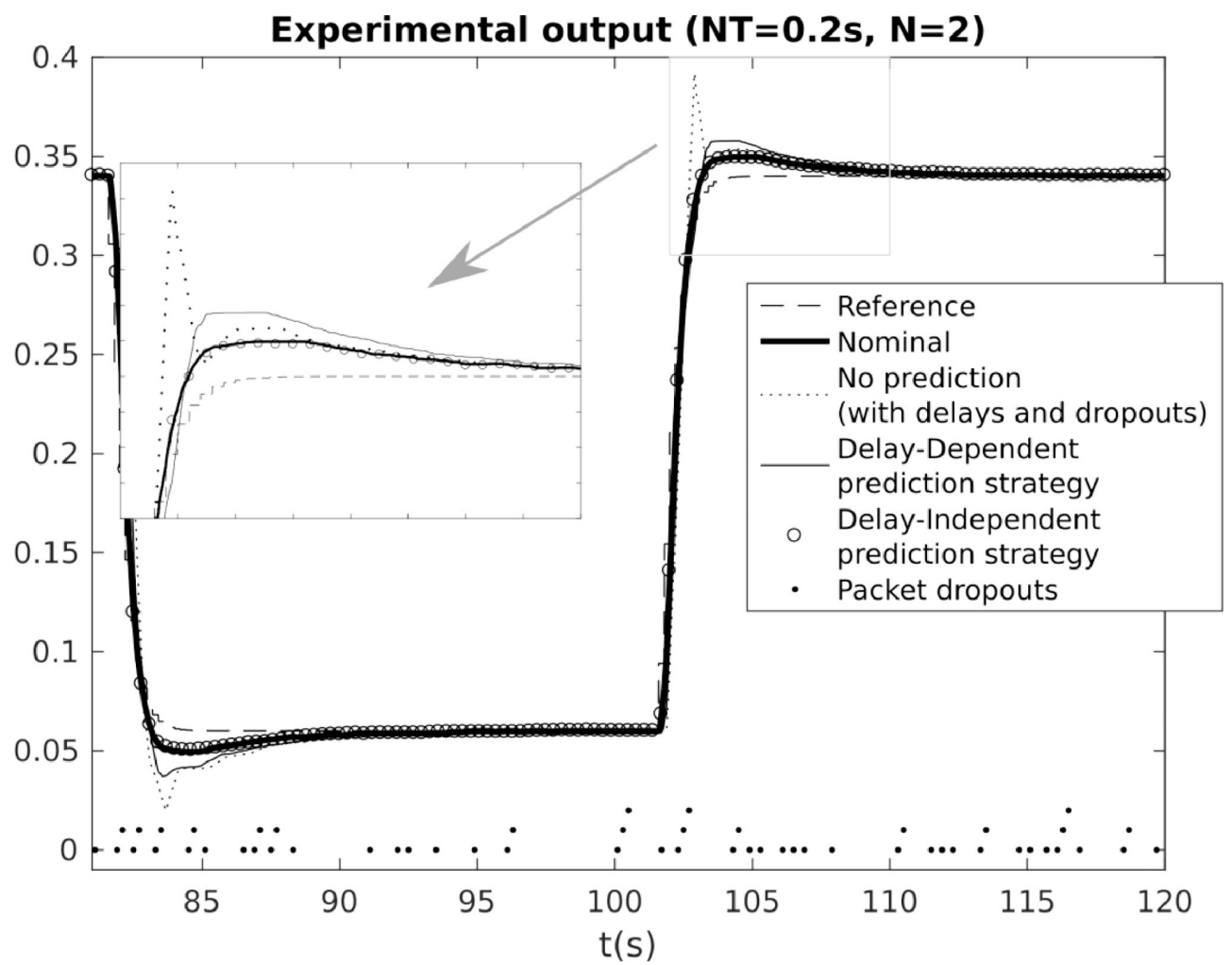

Figure 5.8: Comparison: nominal vs no prediction vs delay-dependent vs delay-independent

is included presents the worst behavior, mainly, when the curves are more pronounced. Once again, the difference between the delay-dependent strategy in [36] and the delay-independent control solution proposed in this work is clearly observed, since, whereas the former does not accurately track the nominal response, the latter does.

\subsection{Conclusions}

In this work, an NCS is presented where time-varying delays, packet dropouts and packet disorder can occur. By means of a novel delay-independent control solution, which integrates packet-based control strategies with dual-rate and predictor-based control techniques, the above problems are faced, and in addi- 


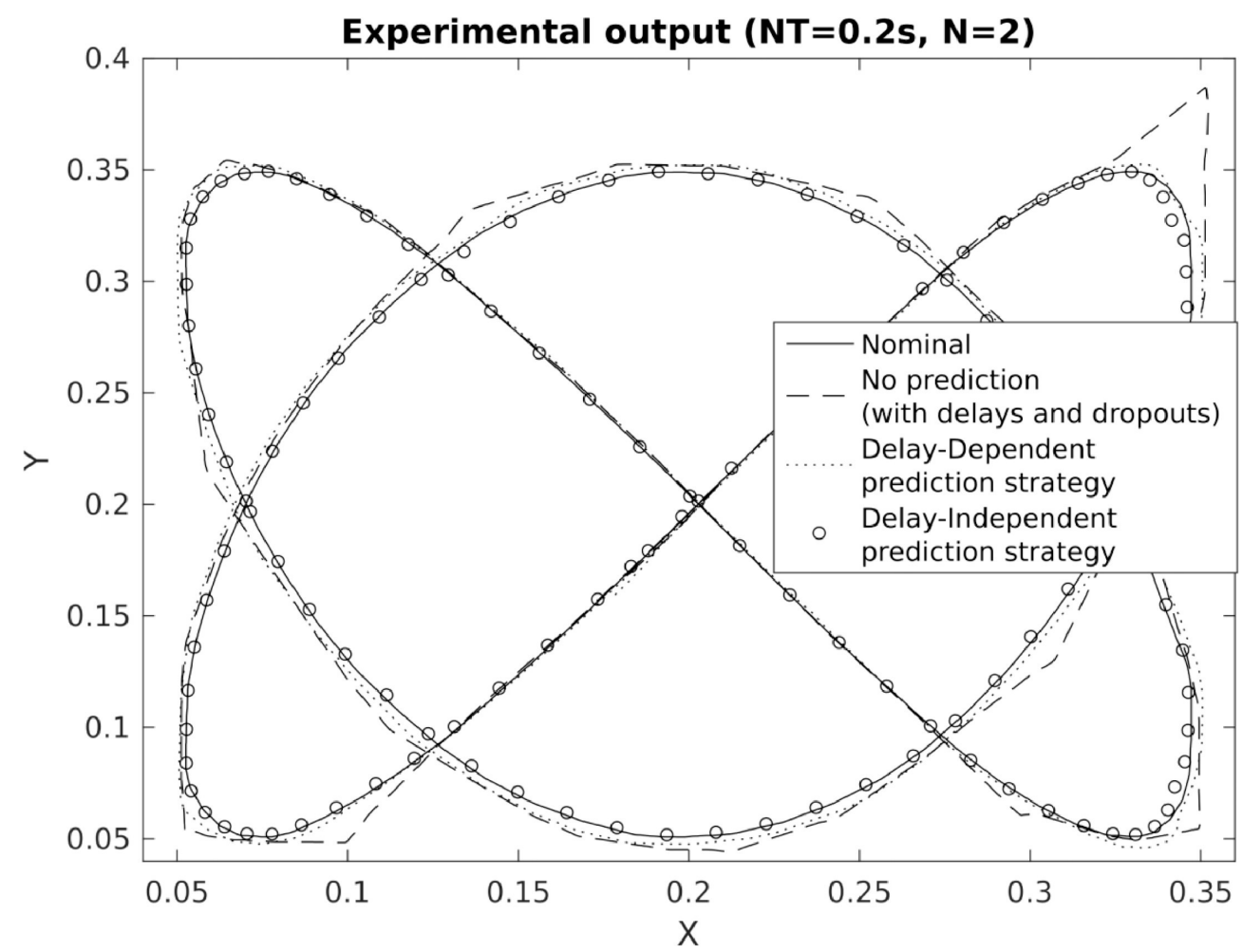

Figure 5.9: Comparison (Lissajous curves): nominal vs no prediction vs delay-dependent vs delay-independent

tion, the usage of the network resources is reduced, while keeping the desired control specifications.

Control system stability is ensured in terms of LMIs. The benefits of the control solution are illustrated by simulation, and experimentally validated by means of a robotic test-bed platform. 


\section{Acknowledgements}

This work is funded by European Commission as part of Project H2020-SEC2016-2017, Topic: SEC-20-BES-2016 - Id: 740736 - "C2 Advanced Multidomain Environment and Live Observation Technologies" (CAMELOT). Part WP5 supported by Tekever ASDS,Thales Research \& Technology, Viasat Antenna Systems, Universitat Politècnica de València, Fundação da Faculdade de Ciências da Universidade de Lisboa, Ministério da DefesaNacional - Marinha Portuguesa, Ministério da Administração Interna Guarda Nacional Republicana. 



\section{References}

[1] Xian-Ming Zhang, Qing-Long Han, and Xinghuo Yu. "Survey on Recent Advances in Networked Control Systems". In: IEEE Transactions on Industrial Informatics 12.5 (Oct. 2016), pp. 1740-1752. ISSN: 1551-3203. DOI: 10.1109/TII.2015.2506545 (cit. on pp. 116, 121).

[2] Dan Zhang et al. "Analysis and synthesis of networked control systems: A survey of recent advances and challenges". In: ISA Transactions 66 (2017), pp. 376-392. ISSN: 0019-0578. DOI: https://doi.org/10.1016/ j.isatra.2016.09.026 (cit. on p. 116).

[3] S. Du, X. Sun, and W. Wang. "Guaranteed Cost Control for Uncertain Networked Control Systems With Predictive Scheme". In: IEEE Transactions on Automation Science and Engineering 11.3 (July 2014), pp. 740748. ISSN: 1545-5955. DOI: 10.1109/TASE.2014.2320322 (cit. on p. 116).

[4] Yu-Long Wang and Qing-Long Han. "Network-based modelling and dynamic output feedback control for unmanned marine vehicles in network environments". In: Automatica 91 (2018), pp. 43-53. ISSN: 0005-1098. DOI: https://doi.org/10.1016/j.automatica.2018.01.026 (cit. on p. 116).

[5] L. Zhang, H. Gao, and O. Kaynak. "Network-Induced Constraints in Networked Control Systems - A Survey". In: IEEE Transactions on Industrial Informatics 9.1 (Feb. 2013), pp. 403-416. ISSN: 1551-3203. DOI: 10.1109/TII.2012.2219540 (cit. on p. 116).

[6] Bao-Lin Zhang and Qing-Long Han. "Network-based modelling and active control for offshore steel jacket platform with TMD mechanisms". In: Journal of Sound and Vibration 333.25 (2014), pp. 6796-6814. ISSN: 0022-460X. DOI: https://doi.org/10.1016/j.jsv.2014.08.007 (cit. on p. 116). 
[7] Xian-Ming Zhang and Qing-Long Han. "Network-based $H_{\infty}$ filtering using a logic jumping-like trigger". In: Automatica 49.5 (2013), pp. 14281435. ISSN: 0005-1098. DOI: https://doi .org/10.1016/j automatica. 2013.01.060 (cit. on pp. 116, 118).

[8] D. Zhang, Q. Han, and X. Jia. "Network-Based Output Tracking Control for a Class of T-S Fuzzy Systems That Can Not Be Stabilized by Nondelayed Output Feedback Controllers". In: IEEE Transactions on Cybernetics 45.8 (Aug. 2015), pp. 1511-1524. ISSN: 2168-2267. DOI: 10.1109/ TCYB. 2014.2354421 (cit. on pp. 116, 118).

[9] Dawei Zhang, Qing-Long Han, and Xinchun Jia. "Network-based output tracking control for T-S fuzzy systems using an event-triggered communication scheme". In: Fuzzy Sets and Systems 273 (2015). Theme: Control Engineering, pp. 26-48. ISSN: 0165-0114. DOI: https ://doi .org/10 . 1016/j.fss.2014.12.015 (cit. on pp. 116, 118).

[10] Dawei Zhang, Zhiyong Zhou, and Xinchun Jia. "Network-based PI control for output tracking of continuous-time systems with time-varying sampling and network-induced delays". In: Journal of the Franklin Institute 355.12 (2018), pp. 4794-4808. ISSN: 0016-0032. DOI: https://doi. org/10.1016/j.jfranklin.2018.04.041 (cit. on p. 116).

[11] Yun-Bo Zhao et al. "Exploring the Different Delay Effects in Different Channels in Networked Control Systems". In: Packet-Based Control for Networked Control Systems: A Co-Design Approach. Singapore: Springer Singapore, 2018, pp. 99-113. ISBN: 978-981-10-6250-6. DOI: $10.1007 /$ 978-981-10-6250-6_8 (cit. on p. 116).

[12] Angel Cuenca et al. "A Non-Uniform Predictor-Observer for a Networked Control System". In: International Journal of Control, Automation and Systems 9.6 (Dec. 2011), pp. 1194-1202. ISSN: 2005-4092. DOI: 10.1007/ s12555-011-0621-5 (cit. on p. 116).

[13] Xiaohua Ge, Fuwen Yang, and Qing-Long Han. "Distributed networked control systems: A brief overview". In: Information Sciences 380 (2017), pp. 117-131. ISSN: 0020-0255. DOI: https://doi.org/10.1016/j.ins . 2015.07.047 (cit. on p. 116).

[14] H. Li and Y. Shi. "Network-Based Predictive Control for Constrained Nonlinear Systems With Two-Channel Packet Dropouts". In: IEEE Transactions on Industrial Electronics 61.3 (Mar. 2014), pp. 1574-1582. ISSN: 0278-0046. DOI: 10.1109/TIE.2013.2261039 (cit. on p. 116). 
[15] A. Liu et al. "Networked filtering with Markov transmission delays and packet disordering". In: IET Control Theory Applications 12.5 (2018), pp. 687-693. ISSN: 1751-8644. DOI: 10.1049/iet-cta. 2017.0755 (cit. on pp. 116, 118).

[16] Andong Liu et al. "New results on stabilization of networked control systems with packet disordering". In: Automatica 52 (2015), pp. 255259. ISSN: 0005-1098. DOI: https://doi.org/10.1016/j.automatica. 2014.12 .006 (cit. on p. 116).

[17] Vicente Casanova et al. "Networked Control Systems: control structures with bandwidth limitations". In: International Journal of Systems, Control and Communications 1.3 (2009), pp. 267-296. DOI: 10.1504/IJSCC. 2009 . 024556. eprint: https : / / www. inderscienceonline.com/doi / pdf/10.1504/IJSCC. 2009.024556 (cit. on p. 116).

[18] Dajun Du et al. "Quantized control of distributed event-triggered networked control systems with hybrid wired?wireless networks communication constraints". In: Information Sciences 380 (2017), pp. 74-91. ISSN: 0020-0255. DOI: https://doi.org/10.1016/j.ins.2016.03.033 (cit. on p. 116).

[19] Ricardo Emerson Julio and Guilherme Sousa Bastos. "A ROS Package for Dynamic Bandwidth Management in Multi-robot Systems". In: Robot Operating System (ROS): The Complete Reference (Volume 2). Ed. by Anis Koubaa. Cham: Springer International Publishing, 2017, pp. 309341. ISBN: 978-3-319-54927-9. DOI: 10 . $1007 / 978-3-319-54927-9 \_10$ (cit. on p. 116).

[20] Yun-Bo Zhao et al. "Packet-Based Control Design for Networked Control Systems". In: Packet-Based Control for Networked Control Systems. Springer, 2018, pp. 15-32 (cit. on p. 116).

[21] Yun-Bo Zhao et al. "Packet-Based Control for Networked Hammerstein Systems". In: Packet-Based Control for Networked Control Systems. PacketBased Control for Networked Control Systems, Sept. 2018, pp. 33-49. ISBN: 978-981-10-6249-0. DOI: 10.1007/978-981-10-6250-6_3 (cit. on p. 116).

[22] Yun-Bo Zhao, Guo-Ping Liu, and David Rees. "Design of a Packet-Based Control Framework for Networked Control Systems". In: IEEE Transactions on Control Systems Technology 17.4 (July 2009), pp. 859-865. ISSN: 1063-6536. DOI: 10.1109/TCST . 2008.2010946 (cit. on p. 116). 
[23] Vicente Casanova et al. "Control of the rotary inverted pendulum through threshold-based communication". In: ISA Transactions 62 (2016). SI: Control of Renewable Energy Systems, pp. 357-366. ISSN: 0019-0578. DOI: https : //doi .org/10.1016/j . isatra. 2016.01.009 (cit. on p. 116).

[24] W. P. M. H. Heemels, M. C. F. Donkers, and A. R. Teel. "Periodic EventTriggered Control for Linear Systems". In: IEEE Transactions on Automatic Control 58.4 (Apr. 2013), pp. 847-861. ISSN: 0018-9286. DOI: 10.1109/TAC.2012.2220443 (cit. on p. 116).

[25] Bao-Lin Zhang, Qing-Long Han, and Xian-Ming Zhang. "Event-triggered $H_{\infty}$ reliable control for offshore structures in network environments". In: Journal of Sound and Vibration 368 (2016), pp. 1-21. ISSN: 0022-460X. DOI: https://doi.org/10.1016/j.jsv.2016.01.008 (cit. on p. 116).

[26] X. Zhang, Q. Han, and B. Zhang. "An Overview and Deep Investigation on Sampled-Data-Based Event-Triggered Control and Filtering for Networked Systems". In: IEEE Transactions on Industrial Informatics 13.1 (Feb. 2017), pp. 4-16. ISSN: 1551-3203. DOI: 10.1109/TII .2016.2607150 (cit. on p. 116).

[27] Lei Zou, Zi-Dong Wang, and Dong-Hua Zhou. "Event-based control and filtering of networked systems: A survey". In: International Journal of Automation and Computing 14.3 (June 2017), pp. 239-253. ISSN: 17518520. DOI: $10.1007 / \mathrm{s} 11633-017-1077-8$ (cit. on p. 116).

[28] Wei Chen and Li Qiu. "Stabilization of networked control systems with multirate sampling". In: Automatica 49.6 (2013), pp. 1528-1537. ISSN: 0005-1098. DOI: https://doi .org/10.1016/j . automatica. 2013.02 . 010 (cit. on p. 116).

[29] Á. Cuenca et al. "Periodic Event-Triggered Sampling and Dual-Rate Control for a Wireless Networked Control System With Applications to UAVs". In: IEEE Transactions on Industrial Electronics 66.4 (Apr. 2019), pp. 3157-3166. ISSN: 0278-0046. DOI: 10.1109/TIE.2018.2850018 (cit. on p. 116).

[30] Julián Salt et al. "A Multirate Control Strategy to the Slow Sensors Problem: An Interactive Simulation Tool for Controller Assisted Design". In: Sensors 14.3 (2014), pp. 4086-4110. ISSN: 1424-8220. DOI: 10.3390/ s140304086 (cit. on pp. 116, 117, 120, 126, 127).

[31] Ángel Cuenca et al. "Non-uniform Multi-rate Estimator based Periodic Event-Triggered Control for resource saving". In: Information Sciences 459 (2018), pp. 86-102. ISSN: 0020-0255. DOI: https ://doi .org/10. 1016/j.ins.2018.05.038 (cit. on p. 116). 
[32] J. Ghommam and F. Mnif. "Predictor-based control for an inverted pendulum subject to networked time delay". In: ISA Transactions 67 (2017), pp. 306-316. ISSN: 0019-0578. DOI: https : // doi .org/10.1016/j . isatra.2017.01.015 (cit. on p. 116).

[33] Yuanqing Xia et al. "Data-driven predictive control for networked control systems". In: Information Sciences 235 (2013). Data-based Control, Decision, Scheduling and Fault Diagnostics, pp. 45-54. ISSN: 0020-0255. DOI: https://doi.org/10.1016/j.ins.2012.01.047 (cit. on p. 116).

[34] Jean-Chrysotome Bolot. "End-to-end packet delay and loss behavior in the Internet". In: ACM SIGCOMM Computer Communication Review. Vol. 23. 4. ACM. 1993, pp. 289-298 (cit. on pp. 116, 117, 121).

[35] Amarnath Mukherjee. "On the dynamics and significance of low frequency components of Internet load". In: Technical Reports (CIS) Dep. of Computer \& Information Science. Univ. Pennsylvania. 1992 (cit. on pp. 116, 117, 121).

[36] Ángel. Cuenca et al. "A packet-based dual-rate PID control strategy for a slow-rate sensing Networked Control System". In: ISA Transactions 76 (2018), pp. 155-166. ISSN: 0019-0578. DOI: https://doi.org/10.1016/ j.isatra.2018.02.022 (cit. on pp. 117, 118, 129, 130, 133, 136-138, 144, 145).

[37] S. Mondié, P. Garcia, and R. Lozano. "Resetting Smith predictor for the control of unstable systems with delay". In: IFAC Proceedings Volumes 35.1 (2002). 15th IFAC World Congress, pp. 77-82. ISSN: 1474-6670. DOI: https : / / doi .org/10 . 3182 / 20020721 - 6-ES-1901 . 00581 (cit. on pp. $117,129,130)$.

[38] S. Mondie, R. Lozano, and J. Collado. "Resetting process-model control for unstable systems with delay". In: Proceedings of the 40th IEEE Conference on Decision and Control (Cat. No.01CH37228). Vol. 3. Dec. 2001, 2247-2252 vol.3. DOI: 10.1109/CDC.2001.980591 (cit. on pp. 117, $129,130)$.

[39] Y. Tipsuwan and Mo-Yuen Chow. "Gain scheduler middleware: a methodology to enable existing controllers for networked control and teleoperation - part I: networked control". In: IEEE Transactions on Industrial Electronics 51.6 (Dec. 2004), pp. 1218-1227. ISSN: 0278-0046. DOI: 10.1109/TIE. 2004.837866 (cit. on pp. 118, 121).

[40] P. Khargonekar, K. Poolla, and A. Tannenbaum. "Robust control of linear time-invariant plants using periodic compensation". In: IEEE Transactions on Automatic Control 30.11 (Nov. 1985), pp. 1088-1096. ISSN: 0018-9286. DOI: 10.1109/TAC.1985.1103841 (cit. on p. 131). 
[41] S. Boyd et al. Linear Matrix Inequalities in System and Control Theory. Studies in Applied Mathematics. Society for Industrial and Applied Mathematics (SIAM, 3600 Market Street, Floor 6, Philadelphia, PA 19104), 1994. ISBN: 9781611970777 (cit. on p. 134).

[42] Hisham A H Al-Khazali and Mohamad R Askari. "Geometrical and Graphical Representations Analysis of Lissajous Figures in Rotor Dynamic System". In: IOSR Journal of Engineering 2 (May 2012). DOI: 10.9790/ 3021-0205971978 (cit. on p. 144). 


\title{
Chapter 6
}

\section{Paper 5. Multirate control strategies for avoid- ing sample losses. Application to UGV path tracking}

\author{
Julián Salt, José Alcaina, Ángel Cuenca, Alfonso Baños
}

When in a digital control strategy there are samples lost due to limitations, different multirate (MR) control options can be assumed for solving the problem: Dual-Rate Inferential Control (IC) and Model-based Dual-Rate Control (MBDR). The objective of this contribution is to analyze and compare both of them trying to assess their behavior under different perspectives. Is an inferential control better than a model-based dual-rate control? These options lead to periodically time-varying discrete-time systems and for this reason lifted modeling is considered. An efficient algorithm is used for computing a MR system frequency response for these control structures. The robust performance and disturbance effects are studied in detail under sample losses and process uncertainty and some considerations are reported. A new QFT (quantitative feedback theory) procedure for dual-rate systems analysis is also described. Analysis and simulation examples and experimental results for $U G V$ path tracking are introduced in this work, indicating that when the model contains important uncertainties the MBDR is a better option.

Keywords: Dual-rate systems; inferential control; model-based control; frequency response; stability; quantitative feedback theory; $U G V$.

ISA Transactions, volume 101, jun 2020, pages 130-146, https: //doi.org/10.1016/j. isatra. 2020.01.025 


\subsection{Motivation}

In computer control of both industrial plants and other systems of a different nature such as precision motion control or mobile robots, it is sometimes difficult to access the output or variable controlled information at all times when it would be ideal to know. Various economic or technical conditions, or failures in the sensor that measures the variable can cause instants of blank data to occur. This loss of samples with respect to the ideal measurement frequency leads to slowly sampled systems. There are several alternatives for the analysis and proposal of solutions in this type of systems. One way is formed by a set of proposals with the objective of supplying the infrequent measure using secondary variables (process quality variables or key indicators) that can be used in the control and that usually entail an estimation of the primary variable. Solutions from the field of control were also introduced, such as cascade control or regulators whose design took into account the problem of lack of data. When the feedback uses estimated outputs, the control scheme is called "inferential control". These kinds of strategies have shown good industrial practical results [1-4]. Therefore, there are two tendencies: one based on measures and the other one on automation that must consider these problems. Sometimes it is not possible to make a rigid classification because there are methods that combine techniques of one or the other alternative. In both tendencies, there are problems that are added to the absence of certain data such as outliers, that is, data located far from the rest of the data, delays, measurement noises and variation the of the system's behavior.

From the point of view of obtaining the main measure directly or indirectly, inferential estimation methods also called soft sensors were considered [5]. Within this option, methods such as model-based assuming the black-box modeling, inferential-based and identification-based have been used. The objective of inferential-based methods is to obtain secondary variables strongly correlated with the quality variables. Identification-based methods consider statistical methods [6] such as partial least squares, principal component analysis, independent component analysis, support vector machines and Bayesian methods [7]. An experimental comparison among some of them, assuming irregular sequence of data, is given in [8].

From the point of view of a control loop, initially the problems of systems behavior variation with time were assumed by contributions from the area of adaptive control. In this sense [9] designed minimum variance controllers for infrequent measure of the output variable in the case of first order systems with delay. These controllers performing with infrequent observations of the 
output were examined making use of the deterministic method introduced by [10]. Some control structures were introduced assuming the use of both primary and secondary variables on nested and cascaded parallel control loops $[11,12]$. Another option was to introduce some procedures to estimate the sampling losses by using a secondary variable $[1,13,14]$. In this case, it is usual to plan an estimator to complete the lost information. The estimator can be considered assuming an input-output [15], or state-space model [16]. This last contribution provides the skeleton so-called "generalized inferential control" for multirate systems separating the design of an estimator and that of a compensator in order to study different $\mathcal{H}_{2}$ optimal controllers in a statespace framework. In $[6,17]$, some tuning rules for the bias update term that appears due to slow or drifting disturbances in the main output variables are proposed considering time delay and multirate sampling in open and closed loop schemes.

Within the option of the control loop assuming the indicated problems, since the end of the 1980s the multirate (MR) option has been considered. An MR can be defined as a hybrid system composed of continuous time elements, usually the plant, and some discrete-time components, usually the controllers and/or the filters, where two or more variables are sampled or updated at different frequencies. If there are only two sampling frequencies, the system is called dual-rate (DR) and an important practical case is the MRIC (multirate input control), that is characterized by a slow output and a fast input, where an integer relation between them and a regular pattern of sampled signals without sampling times mismatch are usually considered. As it is known, it is possible to obtain a linear model from a DR system using the discrete lifting technique as it will be explained later. Although initially MR control was used in the cement industry [18], especially in the kiln [19] due to the different frequencies of chemical processes and material transport, and also in some heat exchanger processes [20], it was in the area of mechanical motion control [21] and robotics manipulators [22] where a higher number of applications were developed. In the DR case there were some attempts to adopt the adaptive control tendency as in [23] and as in contributions of characteristic authors of inferential control $[24,25]$. In recent years it has been a technique widely used in precision robotic manipulators [26], hard disk drive control [27], networked control [28, 29], industrial process [30], in $\mathrm{UxV}$ applications [31, 32] and in mobile robots [28, 33]

The starting point of this work is the simple dual-rate (DR) inferential control strategy introduced in [34]. In that case, with the original control loop including the original digital controller, the lost samples are provided by a process 
model. Only one of each $N$ real closed-loop output samples can be measured. It is a DR control problem that can be approached by another strategy such as designing a nonconventional controller (slow input-fast output) computed from the process model and the original digital controller [35]. Both of them are time-varying discrete-time systems (TVDT). This work attempts to compare these two strategies by means of frequency response (FR) analysis techniques in order to reach a conclusion about the tracking and disturbance robustness in both options. Control techniques based on FR can be adapted to deal with the uncertainty of the process control. QFT is an engineering technique for analysis and design of uncertain feedback systems that uses frequency domain specifications. QFT can be considered as a framework to design robust controllers for processes in which the uncertainty is typically parametric, that is, the process represented by a continuous transfer function $P(s)$ belongs to a family of plants $\mathcal{P}$ whose parameters values are included in certain finite intervals. The FR of all this family is called a "template". The specifications are given in the frequency domain in terms of admissible "bounds" on the FR of the closedloop transfer functions among the different selected inputs and outputs of the closed-loop in order to achieve performance and stability robust specifications by means of a controller $G_{R}(s)$. For a number of frequencies, these specifications are combined with the system uncertainty description for obtaining constraints usually referred to as "boundaries" in the QFT procedure. For each of the selected frequencies, boundaries are given as curves in the Nichols plane, delimiting allowable regions for the open-loop transfer function $G_{R}(j w) P_{0}(j w)$ being $P_{0}$ the nominal process which can be any element in $\mathcal{P}$. Sometimes, an additional precompensator is needed. For a complete introduction to QFT see $[36,37]$. QFT has been successfully applied to a wide variety of practical engineering problems: stable and unstable [38], single-input single-output and multiple-input multiple-output [39], linear and non-linear processes [40] and so on.

Therefore, the contribution of this paper is as follows:

- It proposes two simple and practical DR control strategies to deal with vacant or missing samples (lost by any failure or deliberately lost) in a single-rate control. These alternatives are model-based (dual-rate inferential control and model-based dual-rate control) from the original digital control loop.

- It provides an original QFT-based analysis procedure, never applied to DR systems to the best of the authors' knowledge, to study the effect of mismatch with respect to the real plant (stability and robustness). For this purpose, a new DR FR method based on discrete lifting modeling 
from [41] is used. This is necessary because both options lead to a TVDT system and a proper algebra for its treatment is also introduced.

- It is concluded that the MBDR option is better than the IC one when there are measurement losses, disturbances and the model of the process contains uncertainties.

\subsection{Problem Statement}

The problem to solve is based in a classical digital control with sampling period $T$. From a continuous-time process model $G_{p}(s)$ and planing a desired performance, a digital controller $G_{R}^{T}(z)$ is designed by any suitable direct or indirect method (where variable $z$ stands for the linear time-invariant (LTI)-transform argument at sampling period $T$ ). Under these conditions the control strategy is shown in Figure 6.1. In order to compare it with the other options in this paper, this control will be called "fast" control. $Y^{T}$ is used to describe the z-transform of the sequence $\{y(k T)\}^{1}$.

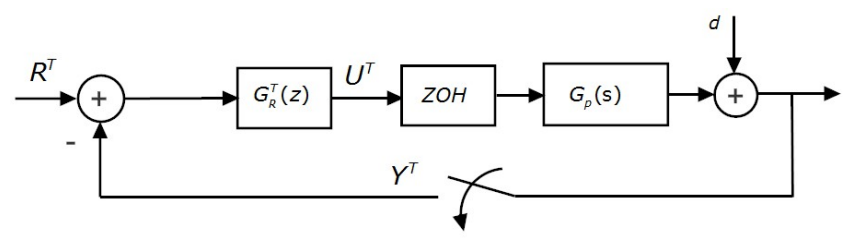

Figure 6.1: Fast digital control

As it was already said, if due to different reasons, it is not possible to have access to all output samples, then different options can be considered. Basically two options will be analyzed in this contribution: the first one is what is known as Dual-rate inferential control (IC) and is shown in Figure 6.2. The second one is known as classical Model-based dual-rate control (MBDR) and will be explained later.

In Figure 6.2, the sampling of the real output provided by a $T$ discrete-time system or a continuous system sampled at period $T$ is known each $N T$ time instants, being $N \in \mathbb{N}$. This situation has been described using a variable $\lambda$ defined as:

\footnotetext{
${ }^{1}$ Similarly $Y^{N T}$ for $\{y(k N T)\}$ will be used. This notation will be assumed for either signals or systems.
} 


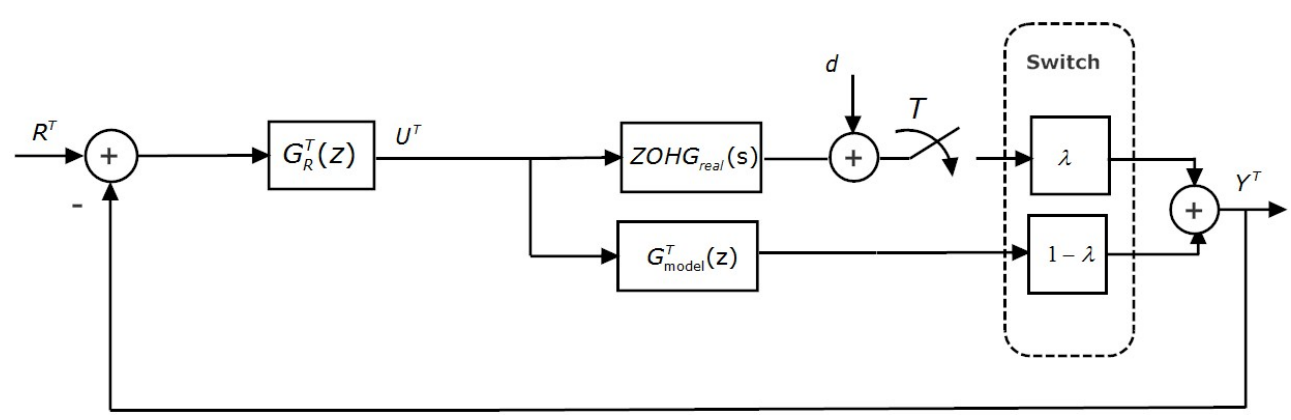

Figure 6.2: Dual-rate inferential control

$$
\lambda=\left\{\begin{array}{ll}
1 & \text { if } t=k N T \\
0 & \text { if } t \neq k N T
\end{array} \quad k \in \mathbb{Z}\right.
$$

$\lambda$ represents a kind of switch that allows to get real samples if $\lambda=1$ or samples each time instants $T$ delivered by a model $G_{\text {model }}^{T}(z)=\mathcal{Z}_{T}\left[Z O H G_{\text {model }}(s)\right]$ in the other case ${ }^{2}$. Obviously a linear periodically time-varying system is met. It is called DR in the sense that two frequencies appear at $1 / T$ and $1 / N T$.

A classical DR solution is introduced in Figure 6.3 where the output is sampled at $N T$ (described by $Y^{N T}$ ) but the control is updated every $T\left(U^{T}\right)$ that is, $N$ times faster. To handle this solution, a special controller can be designed. In [35], a special strategy is proposed. This nonconventional structure controller consists of a slow part $G_{1}^{N T}\left(z^{N}\right)$, a digital hold that repeats the expanded slow controller output $N$ times,

$$
H^{N T, T(z)}=\frac{1-z^{-N}}{1-z^{-1}}
$$

and a fast controller $G_{2}^{T}(z)$. There are different ways to design this DR controller. From the easiest one, that consists in assuming $G_{1}^{N T}=1$ and preserves the fast controller $G_{R}^{T}(z)$ for $G_{2}^{T}$, with unpredictable behavior, to more elaborated design procedures (see for instance [42]). In this contribution, the MBDR controller will be assumed [35]. Basically, if $M(s)$ represents the desired closed loop performance of the original continuous system design:

\footnotetext{
${ }^{2} \mathrm{ZOH}$ is used to denote a zero order hold
} 


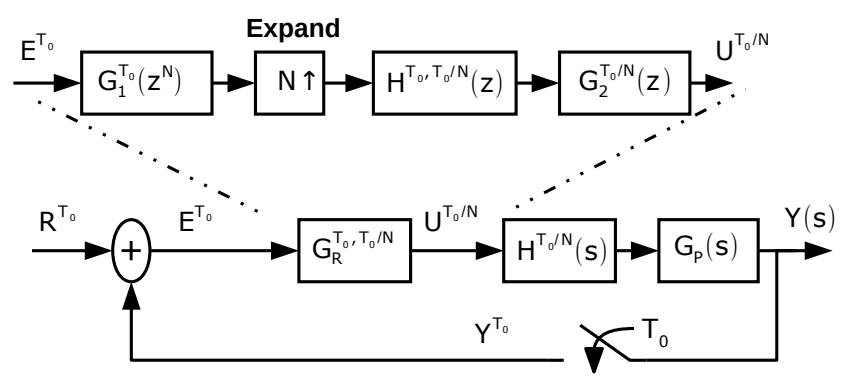

Figure 6.3: Model-based dual-rate control

$$
M(s)=G_{R}(s) G_{p}(s) /\left(1+G_{R}(s) G_{p}(s)\right)
$$

the MBDR will be designed with:

$$
G_{1}^{N T}\left(z^{N}\right)=\frac{1}{1-M^{N T}\left(z^{N}\right)}
$$

and

$$
G_{2}^{T}=M^{T}(z) / G_{p}^{T}(z)
$$

where $M^{T}(z)$ and $M^{N T}\left(z^{N}\right)$ are the $M(s)$ discretized with ZOH for periods $T$ and $N T$ respectively, and $G_{p}^{T}(z)$ is the continuous process model discretized with $\mathrm{ZOH}$ for period $T$. This procedure leads to a behavior that perfectly matches $M(s)$ at the sample points $\{y(k N T)\}$ but sometimes may introduce a ripple between samples due to the magnitude of $N T$. The way to overcome this ripple, if it appears, is as described in [35], considering:

$$
G_{2}^{T}=G_{R}^{T}(z) /\left(1+G_{R}^{T}(z) G_{p}^{T}(z)\right)
$$

being $G_{R}^{T}(z)$ a proper discretization of $G_{R}(s)$ at period $T$. Therefore, the MBDR controller does not cancel the numerator of the process transfer function avoiding the ripple. 


\subsection{Discrete Lifting Modeling}

Some alternatives will now be considered: a fast $T$ digital control, an inferential DR control, and a classical DR control strategy. The last two options introduce a linear periodically TVDT system. Therefore, a procedure to transform it into an LTI system is convenient to make its treatment easier. A well-known method is the discrete lifting modeling. The original idea was introduced by Kranc [43] and it was called vector switch decomposition (VSD). In Figure 6.4, an open loop DR system is shown in which the input and output sequences have different sampling periods, $T_{u}$ and $T_{y}$. It is assumed that they are rationally related. In Figure 6.5, the application of VSD to this case is shown considering the existence of integers $N_{u}, N_{y}$ such that $T_{0}=T_{u} N_{u}=T_{y} N_{y}$ (indeed, then $T_{u} / T_{y}=N_{y} / N_{u}$ is a rational number) being $T_{0}=\operatorname{lcm}\left(T_{u}, T_{y}\right)$, where $l \mathrm{~cm}$ is the least common multiple. This metaperiod $T_{0}$ is the repetition period of every sequence in this scenario. With these conditions it is easy to introduce the discrete lifting operator that will allow an MR control strategy to be transformed into a $T_{0}$ single rate problem. This technique was explained with different details in excellent contributions like [44-46].

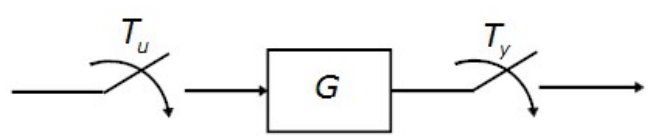

Figure 6.4: Generalized dual-rate system

Kranc's basic idea is explained briefly with the intention to help understand discrete lifting through this perspective. A general sampler $T$ in a certain strategy such that $T_{0}=N T$ ( $T_{0}$ is the $l \mathrm{~cm}$ of all samplers in the strategy) leads to a VSD with $N$ branches. An explanation of this case is exposed introducing the concepts of vectorization and reduction. If $r(t)$ is a continuous signal, with Laplace transform $R(s)$, its sampling at period $T$ leads to the discrete sequence $\{r(k T)\}$ where the $\mathcal{Z}$ function will be noted by $R^{T}$. In these conditions, Kranc's idea was to propose a decomposition of this sampler into $N$ branches, each of them at period $T_{0}=N T$ :

$$
R^{T}=R^{T_{0}}+\left(e^{s T} R\right)^{T_{0}} e^{-s T}+\cdots+\left(e^{s(N-1) T} R\right)^{T_{0}} e^{-s(N-1) T}
$$

So, it is possible to express: 


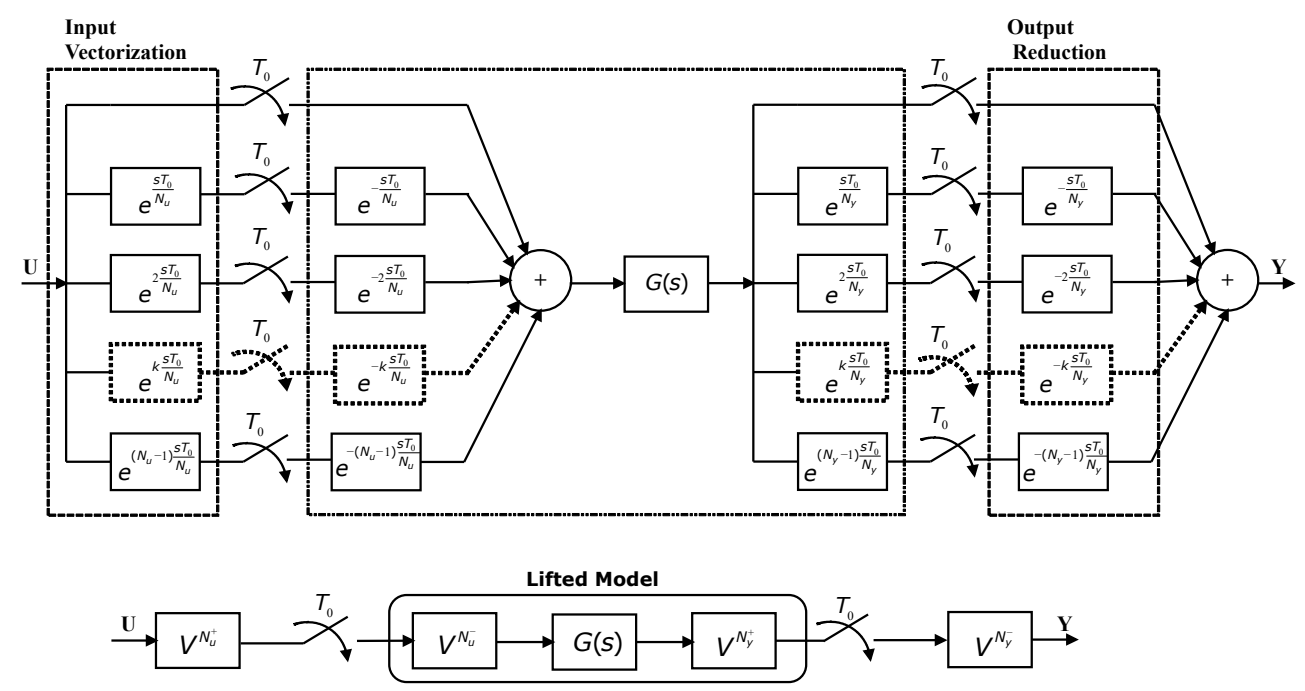

Figure 6.5: Kranc vector switch decomposition. General case

$$
R^{T}=\left[\begin{array}{llll}
1 & e^{-s T} & \cdots & e^{-s(N-1) T}
\end{array}\right]\left(\left[\begin{array}{c}
1 \\
e^{s T} \\
\vdots \\
e^{s(N-1) T}
\end{array}\right] R\right)^{T_{0}}
$$

As seen in Figure 6.5, being in this case $N_{u}=N_{y}=N$ and $T_{u}=T_{y}=T$, the following notation is introduced:

$$
V^{N^{+}}=\left[\begin{array}{c}
1 \\
e^{s T} \\
\vdots \\
e^{s(N-1) T}
\end{array}\right]
$$

for the vectorization operation ${ }^{3}$, and

$$
V^{N^{-}}=\left[\begin{array}{llll}
1 & e^{-s T} & \cdots & e^{-s(N-1) T}
\end{array}\right]
$$

${ }^{3}$ The $e^{m T S}$ blocks are used for off-line analysis purposes. For more explanation see [47] 
for the reduction operation.

This leads to the formula:

$$
R^{T}=V^{N^{-}}\left(V^{N^{+}} R\right)^{T_{0}}
$$

which is the representation of the $T$ sampler decomposition into $N$ branches including a new $T_{0}=N T(\mathrm{lcm})$ sampler. Therefore, with this procedure it is possible to adapt every sampler in a general strategy with regard to a least common multiple $\left(T_{0}\right)$ period of all sampling periods, leading to a multivariable LTI $T_{0}$ single rate system.

If the general case is being treated, then:

$$
\left(\begin{array}{llll}
1 & z^{-N_{y}} & \cdots & z^{-\left(N_{u}-1\right) N_{y}}
\end{array}\right)\left\{\left(\begin{array}{c}
1 \\
e^{s N_{y}} \\
\vdots \\
e^{s\left(N_{u}-1\right) N_{y}}
\end{array}\right) R\right\}^{T_{0}}=V_{d}^{N_{u}^{-}}\left(V^{N_{u}^{+}} R\right)^{T_{0}}
$$

where, as it is reminded, $z$ stands for greatest common divisor $(g c d)$ period $T$ and $T_{0}$ is the least common multiple $(\mathrm{lcm})$ of all sampling periods involved in the general strategy. In equation (6.3), the subindex $d$ (discrete) represents the substitution $z=e^{T s}$. According to this procedure, the strategy in Figure 6.5 can be described as:

$$
Y^{T_{y}}=V_{d}^{N_{y}^{-}} \tilde{G}\left(V^{N_{u}^{+}} U\right)^{T_{0}}
$$

where $\tilde{G}$ is the $T_{0}$ lifted matrix. Note, as it was said before, that every signal is lifted in a $T_{0}$ sampling frame (obviously considering its own sampling period). So, it is common to model the behavior of the DR system characterized via a "lifted" transfer function matrix:

$$
y_{l}\left(z_{T_{0}}\right)=\tilde{G}\left(z_{T_{0}}\right) u_{l}\left(z_{T_{0}}\right)
$$

where the subindex $l$ denotes "lifted" and $z_{T_{0}}$ is referred to the $z$ variable at lcm period $T_{0}$. In equation (6.4), $y_{l}$ is a vector of length $N_{y}, u_{l}$ is a vector of length $N_{u}$ and $\tilde{G}$ is a $N_{y} \times N_{u}$ transfer function matrix [44]. The lengths of the 
vectors are increased in the case of multiple-input and multiple-output (MIMO) systems (multiplied by the number of outputs and inputs, respectively). In Figure 6.5, if the block $G$ between samplers (it could be a continuous process plus zero-order hold) is a single-input single-output LTI system, the system in Figure 6.4 is clearly a shift-varying discrete-time system. In [45] it is proved that the $T_{0}$ lifted matrix $\tilde{G}=V_{d}^{N_{y}^{+}} G V_{d}^{N_{u}^{-}}$is LTI $N_{u}$ input $N_{y}$ output shiftinvariant. This correspondence between periodic and expanded LTI systems preserves both the analytic and algebraic properties of the systems [48]. In particular, block-diagram algebra procedures could be considered ${ }^{4}$. In the next section, this algebra will be introduced to model the periodic systems to be compared.

Note that in Figure 6.5, $T_{0} / N_{u}=N_{y} T$ and $T_{0} / N_{y}=N_{u} T$. It is possible to prove using Mason's rule applied to this strategy, that the central part of the diagram would be:

$$
\left(\begin{array}{llll}
1 & z^{-N_{u}} & \cdots & z^{-\left(N_{y}-1\right) N_{u}}
\end{array}\right) \frac{\tilde{G}\left(z^{N_{y} N_{u}}\right)}{N_{y}}\left(\begin{array}{c}
1 \\
z^{N_{y}} \\
\vdots \\
z^{\left(N_{u}-1\right) N_{y}}
\end{array}\right)
$$

At this point, it is important to point out that the discrete-lifted operators can be expressed in an external [45] or internal [49] representation. In the contribution [46], the links between both of them were described. In the next subsection, two different examples are developed in detail.

\subsubsection{Examples of discrete lifted systems}

There are different ways to obtain the lifted model. For processes, the easiest one may be to consider the successive iterations from the $g c d T$ discrete statespace. In this work, two different options will be considered: a continuous-time system preceded by a zero-order hold and a pure discrete system performing at the input sampling period but with a different output sampling period.

If a strictly proper continuous system is discretized (assuming $\mathrm{ZOH}$ ) at period $T$, with a realization $(A, B, C, 0)$, then the lifted dual-rate model has a realization $\left(A_{l}, B_{l}, C_{l}, D_{l}\right)$, at metaperiod $T_{0}$, where these matrices are obtained

\footnotetext{
${ }^{4}$ It must be noted that it is also possible to define the lifted strategy from a discrete system, but obviously the input sampling period will be the same as the one applied to the system
} 
by repeated evaluations of the equations at sampling period $T$ giving rise to well-known convolution-like formulae. For instance,

$$
\begin{aligned}
y\left(k T_{0}+\zeta T\right)= & C x\left(k T_{0}+\zeta T\right)= \\
= & C\left[A^{\zeta} x\left(k T_{0}\right)+A^{\zeta-1} B u\left(k T_{0}\right)+\right. \\
& \left.+A^{\zeta-2} B u\left(k T_{0}+T\right)+\cdots+B u\left(k T_{0}+(\zeta-1) T\right)\right]
\end{aligned}
$$

for $\zeta=1, \cdots,\left(N_{u}-1\right) N_{y}$. However, the zero-order-hold entails

$$
\begin{aligned}
& u\left(k T_{0}+d N_{y} T\right)=u\left(k T_{0}+\left(d N_{y}+1\right) T\right)=\cdots=u\left[k T_{0}+\left((d+1) N_{y}-1\right) T\right] \\
& \forall d=0,1 \cdots,\left(N_{u}-1\right)
\end{aligned}
$$

Therefore, the lifted matrices are obtained by suitably stacking the results from the above equation. As it was said before, it is also possible to consider that $G$ may have different natures (continuous system without $\mathrm{ZOH}$, digital system, etc). For each of these cases, proper input or output assignments (like (6.7)) must be established leading to a specific lifted model [49].

Now, a brief example can be considered. If in Figure $6.4, G$ is a continuoustime plant with ZOH and $T_{u}=0.3$ and $T_{y}=0.2\left(N_{u}=2\right.$ and $\left.N_{y}=3\right)$, then the $l c m T_{0}=0.6$, and the $g c d T=0.1$. For this case, the lifted representation $(\tilde{A}, \tilde{B}, \tilde{C}, \tilde{D})$ would be:

$$
\begin{gathered}
x\left[(k+1) T_{0}\right]=A^{6} x\left(k T_{0}\right)+\left(\begin{array}{ll}
\left(A^{5}+A^{4}+A^{3}\right) B & \left(A^{2}+A+I\right) B
\end{array}\right)\left(\begin{array}{c}
u\left(k T_{0}\right) \\
u\left(k T_{0}+3 T\right)
\end{array}\right) \\
\left(\begin{array}{c}
y\left(k T_{0}\right) \\
y\left(k T_{0}+2 T\right) \\
y\left(k T_{0}+4 T\right)
\end{array}\right)=\left(\begin{array}{c}
C \\
C A^{2} \\
C A^{4}
\end{array}\right) x\left(k T_{0}\right)+\left(\begin{array}{cc}
0 & 0 \\
C(A+I) B & 0 \\
C\left(A^{3}+A^{2}+A\right) B & C B
\end{array}\right)\left(\begin{array}{c}
u\left(k T_{0}\right) \\
u\left(k T_{0}+3 T\right)
\end{array}\right)
\end{gathered}
$$

In the case of a discrete input without zero-order hold, equation (6.6) is valid, but now the difference is that the input is not held. So, in equation (6.7):

$$
\begin{aligned}
& u\left(k T_{0}+d N_{y} T\right) \quad \text { has a value } \\
& u\left(k T_{0}+\left(d N_{y}+1\right) T\right)=\cdots=u\left[k T_{0}+\left((d+1) N_{y}-1\right) T\right]=0 \\
& \forall d=0,1, \cdots,\left(N_{u}-1\right)
\end{aligned}
$$


As an example, the same case that was described in equation (6.8), gives

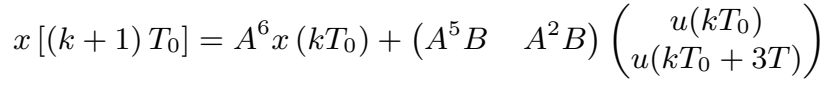

$$
\begin{aligned}
& \left(\begin{array}{c}
y\left(k T_{0}\right) \\
y\left(k T_{0}+2 T\right) \\
y\left(k T_{0}+4 T\right)
\end{array}\right)=\left(\begin{array}{c}
C \\
C A^{2} \\
C A^{4}
\end{array}\right) x\left(k T_{0}\right)+\left(\begin{array}{cc}
0 & 0 \\
C A B & 0 \\
C A^{3} B & C B
\end{array}\right)\left(\begin{array}{c}
u\left(k T_{0}\right) \\
u\left(k T_{0}+3 T\right)
\end{array}\right)
\end{aligned}
$$

In these examples, the lifted matrix $\tilde{G}$ is the quadruple composed by $\tilde{A}, \tilde{B}, \tilde{C}, \tilde{D}$ or its external equivalent description.

\subsection{Dual-rate systems frequency domain}

In this section, a description of the frequency domain procedures used for the analysis comparison of both strategies is introduced. First, a new efficient algorithm [41] for computing the DR systems frequency response is shown and how to understand this algorithm is also described. The objective is to analyze the robust stability and robust tracking of different systems with the options considered in this work. The option selected for a unified analysis is the quantitative feedback theory (QFT) [36, 50].

\subsubsection{DR Systems Frequency Response Computation}

From a lifted LTI representation of a DR system, it is possible to calculate the frequency response. The theorem introduced in [41] says:

Theorem: The output $y(k)$, when $u(k)=e^{j \omega T_{u} k}$, of a SISO dual-rate $\left(N_{u} T_{u}=N_{y} T_{y}\right)$ lifted system $y_{l}(z)=G_{\text {lifted }}(z) u_{l}(z)$ is a collection of components $y_{r}(k)=\bar{y}_{r} e^{j T_{y} \omega_{r} k}$ of frequencies $\omega_{r}=\omega+2 \omega_{y}^{s} r / N_{y}$, for $r=0, \ldots,\left(N_{y}-1\right)$, with $\omega_{y}^{s}=\pi / T_{y}$, and $\bar{y}_{r}$ is given by:

$$
\bar{y}_{r}=\frac{1}{N_{y}} \sum_{p=0}^{N_{y}-1} \sum_{q=0}^{N_{u}-1} G_{p q} e^{j \omega_{r} T_{y} N_{y}} e^{-j T_{y} \omega_{r} p} e^{j \omega T_{u} q}
$$

As it was proved in [41], the discrete frequency response computations are carried out by replacing $z=e^{j \omega T}$ for some $T$. It is easy to check that, from (6.11), the components will be given by the product of the frequency response 
of a left factor $\left[1 z^{-1} z^{-2} \ldots z^{-\left(N_{y}-1\right)}\right] G_{l}\left(z^{N_{y}}\right)$ replacing $z=e^{j \omega_{r} T_{y}}$, which gives a row vector, and the right factor (column vector) $\left(1 z z^{2} \ldots z^{N_{u}-1}\right)^{T}$ replacing $z=e^{j \omega T_{u}}$.

As it will be explained later, there is a possibility of computing the whole frequency response from only one Bode plot in the case $N_{y}$ and $N_{u}$ are coprime. Note that, $\omega_{s}=2 \pi /\left(N_{y} T_{y}\right)$ and then that the components of the frequency response are defined at $\omega_{r}=\omega+r \omega_{s}$, for, $r=0, \cdots, N_{y}-1$. For more details, see [41]

\subsubsection{Frequency Response Interpretation}

Considering the equation (6.5) and doing the multiplication of those matrices, an usually high dimension single-input single-output transfer function is obtained; the problem is even worse if the $l \mathrm{~cm}\left(T_{u}, T_{y}\right)$ is a big number. This is a simple procedure but it produces very unsatisfactory results applying usual computer aided design (CAD) FR routines as it is proved in [51] and that is why a new efficient algorithm for DR systems FR was introduced. The results from the algorithm proposed in the previous section are accurate but require an interpretation. From the results above, it can be tested that the DR system frequency response can be explained by adding $\frac{N_{y}}{\operatorname{gcd}\left(N_{u}, N_{y}\right)}$ values from the Bode diagram outlined from 0 to $\left(\frac{N_{y}}{\operatorname{gcd}\left(N_{u}, N_{y}\right)}-1\right) N_{u} w_{s}$. In fact, for an input frequency $b \mathrm{Rad} / \mathrm{s}$, the output is the sum of sine signals with frequencies $b, b+N_{u} w_{s}$, $b+2 N_{u} w_{s}, \cdots b+\left(\frac{N_{y}}{\operatorname{gcd}\left(N_{u}, N_{y}\right)}-1\right) N_{u} w_{s}$ with amplitude and phase determined by Bode diagram at the indicated points. The coprime case is illustrative and gives a link with the theoretical results. If $T_{u}$ and $T_{y}$ are coprime, then, considering the blocks diagram from Figure 6.5, the DRS frequency response is obtained reading the $N_{y}$ points from the magnitude and phase Bode diagrams in the interval from 0 to $N_{u} N_{y} w_{s}$, each one separated a length of $N_{u} w_{s}$ from the previous point.

The proposed algorithm introduced in [41] returns $N_{y}$ Bode diagrams related to the tracks: 0 to $N_{u} N_{y} w_{s}, N_{u} w_{s}$ to $\left(N_{y} N_{u}+N_{u}\right) w_{s}, \cdots,\left(N_{y}-1\right) N_{u} w_{s}$ to $\left(N_{y} N_{u}+\left(N_{y}-1\right) N_{u}\right) w_{s}$. So, the same Bode translated $N_{u} w_{s} N_{y}$ times is reached. Of course, the $N_{y}$ points can be read in the interval 0 to $N_{u} w_{s}$ knowing the true sense of all of them.

Finally, it must be noted that the addition of these $N_{y}$ components will not be a pure sine signal. 


$$
\begin{aligned}
y\left(k T_{y}\right)= & A_{1} \sin \left(w k T_{y}+\varphi_{1}\right)+A_{2} \sin \left(\left(w+N_{u} w_{s}\right) k T_{y}+\varphi_{2}\right)+\cdots+ \\
& +A_{N_{y}} \sin \left(\left(w+\left(N_{y}-1\right) N_{u} w_{s}\right) k T_{y}+\varphi_{N_{y}}\right)
\end{aligned}
$$

However if the decomposition of each component is observed, it is possible to obtain a $T_{0}$ approximation that makes the addition a sinusoidal function. In effect, considering:

$$
\begin{aligned}
A_{\nu} \sin \left(w_{\nu} k T_{y}+\varphi_{\nu}\right)= & A_{\nu} \sin \left(w_{\nu} k T_{0}+\varphi_{\nu}\right)+ \\
& z_{T_{y}}^{-1} A_{\nu} \sin \left(w_{\nu}\left(k T_{0}+T_{y}\right)+\varphi_{\nu}\right)+\cdots \\
& z_{T_{y}}^{-\left(N_{y}-1\right)} A_{\nu} \sin \left(w_{\nu}\left(k T_{0}+\left(N_{y}-1\right) T_{y}\right)+\varphi_{\nu}\right)+ \\
& \text { for } \nu=1, \cdots,\left(N_{y}-1\right) \\
A_{\nu} \sin \left(w_{\nu} k T_{y}+\varphi_{\nu}\right)= & A_{\nu} \sin \left(w_{\nu} k T_{0}+\varphi_{\nu}\right)+ \\
& z_{T_{y}}^{-1} A_{\nu} \sin \left(w_{\nu} k T_{0}+\left(w_{\nu} T_{y}+\varphi_{\nu}\right)\right)+\cdots \\
& z_{T_{y}}^{-\left(N_{y}-1\right)} A_{\nu} \sin \left(w_{\nu} k T_{0}+\left(w_{\nu}\left(N_{y}-1\right) T_{y}+\varphi_{\nu}\right)\right)+ \\
& \text { for } \nu=1, \cdots,\left(N_{y}-1\right)
\end{aligned}
$$

So, adding the contributions of each component at $k T_{0}$, that is the $T_{0}$ resampling of the sequence $y\left(k T_{y}\right)$ :

$$
\begin{aligned}
y^{T_{0}}\left(k T_{y}\right) & =A_{1} \sin \left(w k T_{0}+\varphi_{1}\right)+ \\
& +A_{2} \sin \left(w+w_{s}\right) k T_{0}+\left(\left(w+w_{s}\right) T_{y}+\varphi_{\nu}\right)+\cdots \\
& +A_{N_{y}} \sin \left(\left(\left(w+\left(N_{y}-1\right) w_{s}\right)+\left(N_{y}-1\right) N_{u} w_{s}\right) k T_{0}+\varphi_{N_{y}}\right)
\end{aligned}
$$

Finally leads to the $T_{0}$ approximation:

$$
\begin{aligned}
y^{T_{0}}\left(k T_{y}\right) & =A_{1} \sin \left(w k T_{0}+\varphi_{1}\right)+ \\
& +A_{2} \sin \left(w k T_{0}+\left(w_{s} T_{0}+\varphi_{\nu}\right)\right)+\cdots \\
& +A_{N_{y}} \sin \left(w k T_{0}+\left(N_{y}-1\right) w_{s} T_{0}+\varphi_{N_{y}}\right)
\end{aligned}
$$

Therefore, as it was said, a sinusoidal approximation is obtained. 


\subsubsection{Dual-rate systems quantitative feedback theory}

Obviously, this work does not try to expose or revisit the quantitative feedback theory (QFT) $[36,50]$. The purpose is just to introduce its application to a DR system. The starting point is [52] that introduces the QFT for direct digital control.

In the present work, the QFT analysis methodology is slightly different than usual. Classically, as it was introduced in section 1, QFT analysis and design involves the mapping of robust specifications (robust stability, robust disturbance rejection, control effort, robust tracking among others) into certain bounds on a nominal loop transmission followed by loop shaping for some selected frequencies. These bounds split the complex plane into two areas where the open loop transfer function should lie inside one. In this work QFT is used as analysis tool. The controller design (IC and MBDR) is firstly performed considering a nominal plant model and design specifications (relative stability with some stability margins, disturbance rejection, ...). For this nominal design, QFT is used for computing restrictions (boundaries) to be satisfied by the nominal open-loop gain, for different working frequencies (note that templates are reduced to a point in the Nichols plane). Once the nominal controller design is performed, it is considered the effect of plant uncertainty on the satisfaction of (closed-loop) design specifications. This is done by computing the open-loop gain corresponding to the plant with uncertainty, and checking if its FR satisfies the previously computed boundaries. For all these calculations is absolutely necessary the DR FR introduced before and the algebra with lifted blocks that will be explained in section 6.5.

In this work two of the specifications are considered but obviously the procedure can be applied for all the options. Now the gain (GM) and phase margins $(\mathrm{PM})$, and the discrete-time output disturbance rejection are considered. In both cases and with respect to a structure like the one shown in Figure 6.1, some restriction must be planned in the closed loop transfer function denominator [52]. In the case of robust stability margins $\left|1+G_{p}(z) G_{R}(z)\right| \geq \mu$ with $z=$ $e^{j w T}, w \in\left[0, w_{s} / 2\right]$ being $G M=(1-\mu)^{-1}$ and $P M=180^{\circ}+2 \cos ^{-1}(\mu / 2)$. For the output disturbance rejection can be assumed $\left|1+G_{p}(w) G_{R}(w)\right| \geq(1 / \delta(w))$. In the applications, section 6.6 and section 6.7 , these restrictions will be considered more specifically. 


\subsection{Closed-loop lifted models}

In this section, the block-diagram (obtained by lifting) algebra is used to show the models of the closed loops to be compared. Obviously, it is not necessary to obtain the fast digital control lifted model. The closed loop $Y$ vs $R$ for MBDR and IC cases are shown in Figure 6.6 and Figure $6.7^{5}$. These figures show the complete evolution from the initial digital control strategy to the equivalent one using lifted signals. The substitution of each sampler following Kranc's idea (vector switch decomposition) is the first step. A reference sampled at whatever $T_{\text {ref }}$ and an output sampled at $T_{3}=T_{0} / N_{3}$ with $N_{3}$ high enough have been added to the original strategies. Note that the sampling for feedback purposes is only possible at $T_{1}=T_{0} / N_{1}$, that is, a low magnitude. $T_{3}$ reflects open-loop intersampling. It is not realistic, but it is included with the intention to define a general problem. As it can be seen due to the reasons introduced in subsection 6.3.1, in a middle stage kranczoh or krancdig are differentiated corresponding to lifting matrices of $\mathrm{ZOH}$ plus continuous process and digital controller respectively. Then the notation $K_{\text {type }} G^{N_{u}, N_{y}}$ is considered where the superindex is referred to the multiplicity of input and output regarding the $l \mathrm{~cm} T_{0}$ period, and the subindex is related to the kind of operator. In this notation, the transfer function $G$ is also added for easy understanding. In Figure 6.6 and Figure 6.7, the input control is updated every $T_{2}=T_{0} / N_{2}$. Note that $N_{3} \geq N_{2} \geq N_{1}$.

\footnotetext{
${ }^{5}$ Note that in these figures the pure zero-order hold is shown as $Z O H G_{\text {unit }}(s)$ being $G_{\text {unit }}(s)=1$
} 


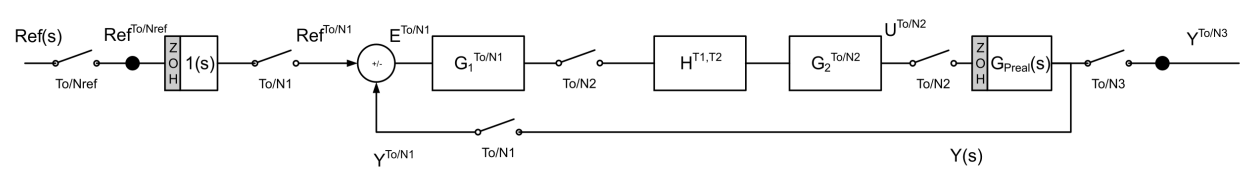

(a) Original diagram

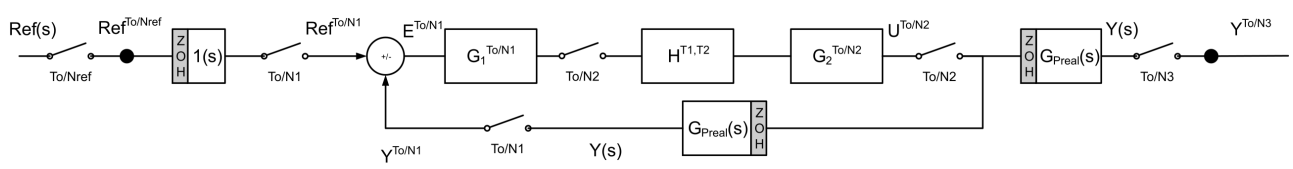

(b)

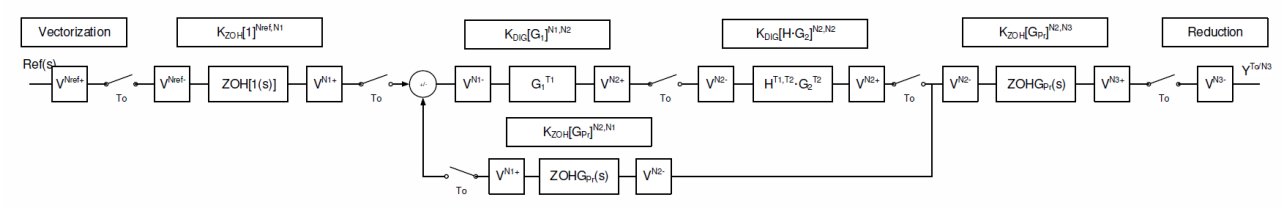

(c)

Figure 6.6: $Y / R$ MBDR lifted process 


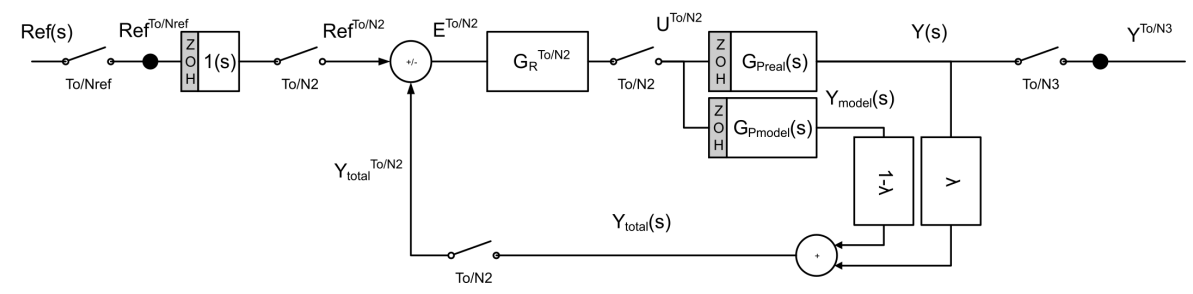

(a) Original diagram

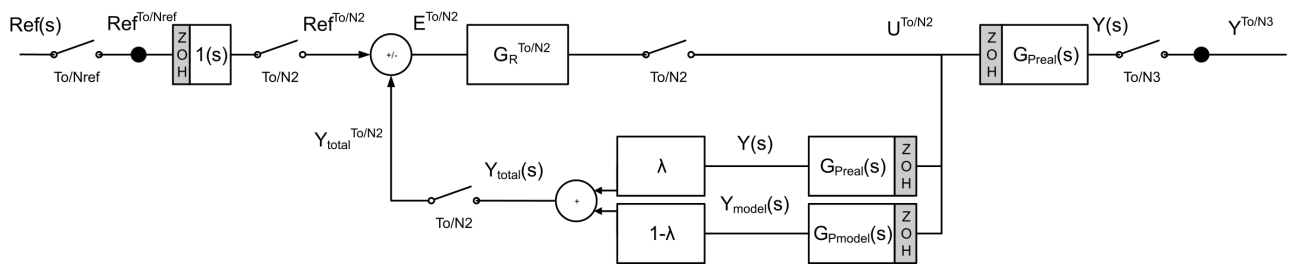

(b)

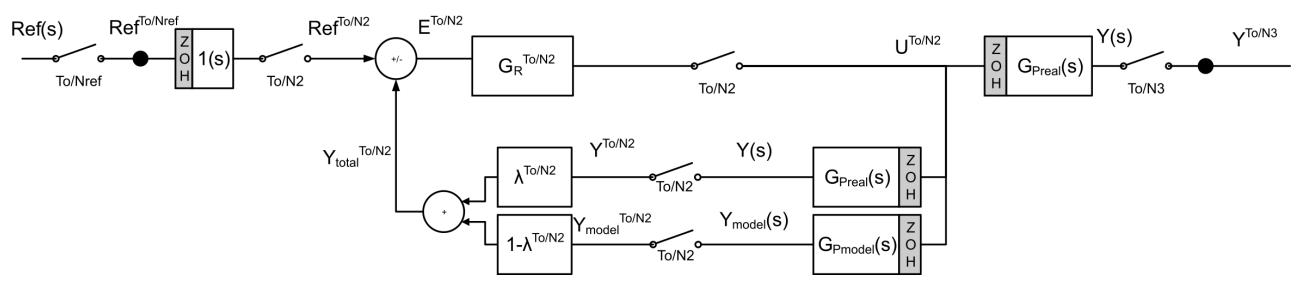

(c)

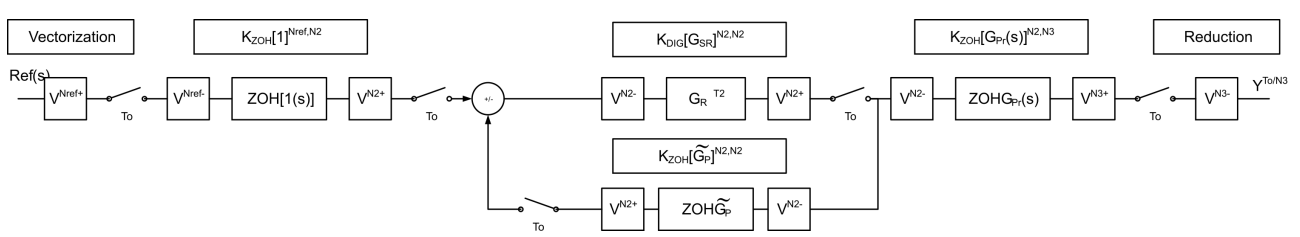

(d)

Figure 6.7: Y/R IC lifted process

Analyzing these figures and the procedure explained graphically, it is possible to express the lifted input-output relation for the MBDR loop (6.12) and for the IC Loop (6.13). Note that in this last case, IC, the model has been lifted at the metaperiod. In this last case, the switch selector which models the lost 
information and the complementary information delivered by the model leads to a system with periodicity $T_{0}$.

For the case of MBDR strategy:

$$
\begin{aligned}
\bar{U}= & K_{D I G} G_{2}^{N_{2}, N_{2}} \times K_{D I G} G_{1}^{N 1, N 2} \times \\
& \times\left[I_{\alpha_{1} \times \alpha_{2}}+K_{Z O H} G_{p r e a l}^{N_{2}, N_{1}} \times K_{D I G} G_{2}^{N_{2}, N_{2}} \times K_{D I G} G_{1}^{N 1, N 2}\right]^{-1} \\
Y^{T / N_{3}}= & K_{Z O H} G_{\text {preal }}^{N_{2}, N_{3}} \times \bar{U} \\
Y^{T / N_{1}}= & K_{Z O H} G_{\text {preal }}^{N_{2}, N_{1}} \times \bar{U}
\end{aligned}
$$

where $\alpha_{1} \times \alpha_{2}$ is the size of the product $K_{Z O H} G_{p}^{N_{2}, N_{1}} \times K_{D I G} G_{2}^{N_{2}, N_{2}} \times$ $K_{D I G} G_{1}^{N 1, N 2}$ and the notation $I$ is used for the identity matrix.

For the case of IC strategy:

$$
\begin{aligned}
\bar{U}_{R} & =K_{D I G} G_{R}^{N_{2}, N_{2}} \times\left[I_{\beta_{1} \times \beta_{2}}+\tilde{K}_{Z O H} G_{p}^{N_{2}, N_{2}} \times K_{D I G} G_{R}^{N_{2}, N_{2}}\right]^{-1} \\
Y^{T / N_{3}} & =K_{Z O H} G_{p r e a l}^{N_{2}, N_{3}} \times \bar{U}_{R} \\
Y^{T / N_{1}} & =K_{Z O H} G_{\text {preal }}^{N_{2}, N_{1}} \times \bar{U}_{R}
\end{aligned}
$$

being $\beta_{1} \times \beta_{2}$ the size of the product $\tilde{K}_{Z O H} G_{p}^{N_{2}, N_{2}} \times K_{D I G} G_{R}^{N_{2}, N_{2}}$

Now, $\tilde{K}_{Z O H} G_{p}^{N_{2}, N_{2}}$ is quite special because it is made up of two different parts which correspond to the order of sampling in the switch of Figure 6.2. This will be easier to understand through an example.

\subsubsection{Example}

Take, for instance, $N=3$ and assume that only the first of a set of $N$ samples is picked up from the real process $G_{\text {preal }}$ and the other $N-1$ are delivered by the model $G_{\text {pmodel }}$. If both of them are second-order systems and described by a state representation $A_{r}, B_{r}, C_{r}, D_{r}$ and $A_{m}, B_{m}, C_{m}, D_{m}$, then $\tilde{K}_{Z O H} G_{p}^{N, N} \equiv$ $A_{t}, B_{t}, C_{t}, D_{t}$. Some auxiliary matrices are defined to simplify the notation ${ }^{6}$ :

\footnotetext{
${ }^{6}$ a $N, N$ is assumed in order to simplify the explanation. That is, $N_{2}=N_{3}=N$ which is not relevant for the analysis procedure
} 


$$
\begin{aligned}
& \chi_{1}=\left[\begin{array}{l|l}
I_{\operatorname{dim} A_{r}} & 0 \\
\hline 0 & 0_{\operatorname{dim} A_{m}}
\end{array}\right] \quad \chi_{2}=\left[\begin{array}{l|l}
0_{\operatorname{dim} A_{r}} & 0 \\
\hline 0 & I_{\operatorname{dim} A_{m}}
\end{array}\right] \\
& \chi_{3}=\left[\begin{array}{l|l}
1_{1 \times 1} & 0_{1 \times N-1} \\
\hline 0_{N-1 \times 1} & 0_{N-1 \times N-1}
\end{array}\right] \quad \chi_{4}=\left[\begin{array}{l|l}
0_{1 \times 1} & 0_{1 \times N-1} \\
\hline 0_{N-1 \times 1} & 1_{N-1 \times N-1}
\end{array}\right] \\
& A_{t}=\left[\chi_{1} \times A_{r}+\chi_{2} \times A_{p}\right] \\
& B_{t}=\left[\frac{B_{r}}{B_{m}}\right] \\
& C_{t}=\left[\chi_{3} \times C_{r} \mid \chi_{4} \times C_{m}\right] \\
& D_{t}=\left[\frac{\chi_{3} \times D_{r}}{\chi_{4} \times D_{m}}\right]
\end{aligned}
$$

that is, the state equation is like a parallel structure of two systems, in which the first output in a metaperiod is obtained by means of the real process and the rest are obtained using the model. Therefore, the $C_{t}$ and $D_{t}$ matrices are composed by rows regarding each sample.

The next section analyses the reference tracking problem and the disturbance rejection in each of the introduced schemes as well. In order to study the same kind of disturbances for all of them, a continuous $d$ sampled at $T_{0} / N_{d}$ has been assumed. It is observed the application to the real process although the design procedure was based on the process model. Figure 6.8 and Figure 6.9 describe the lifted diagram for MBDR and IC cases, respectively. 
Chapter 6. MR control strategies for avoiding sample losses. Application to UGV path tracking

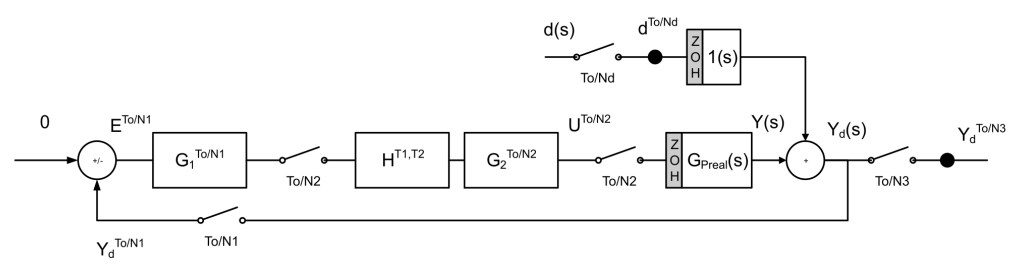

(a) Original diagram

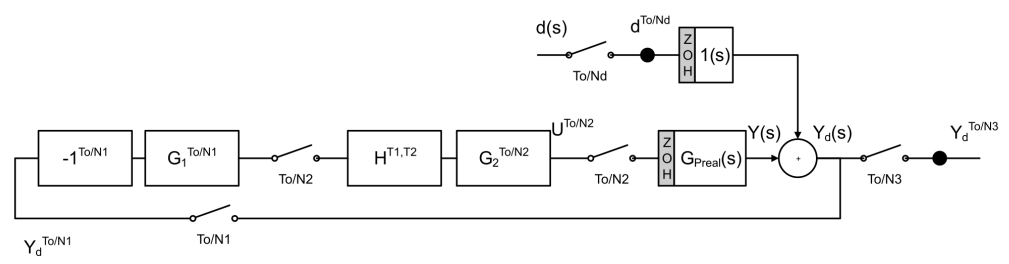

(b)

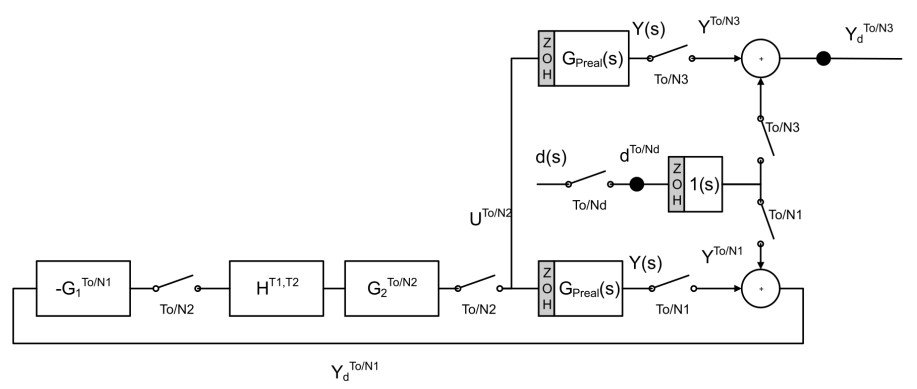

(c)

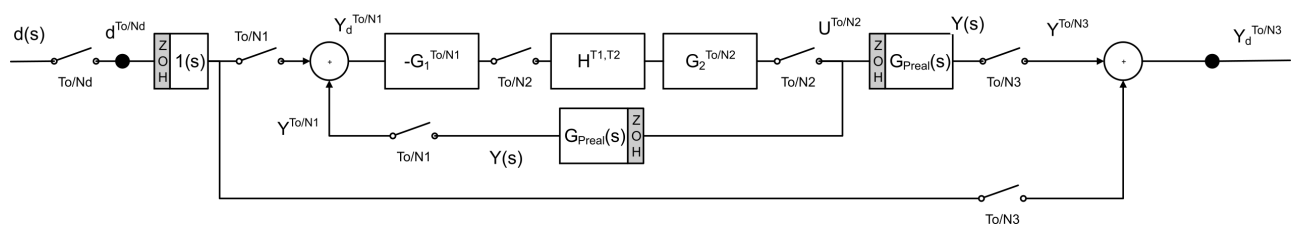

(d)

$\overline{176}$ 


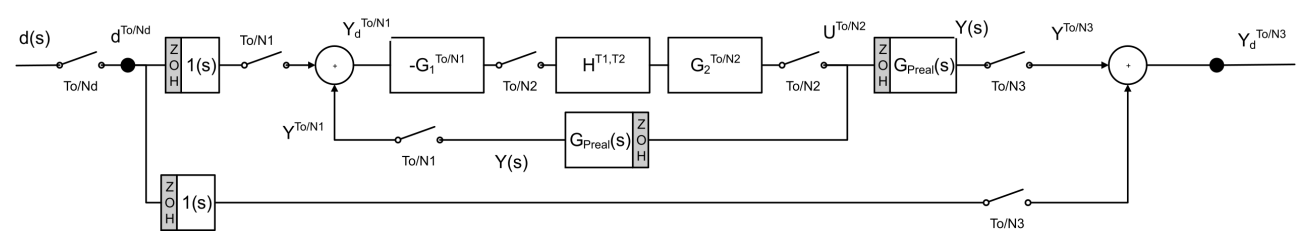

(e)

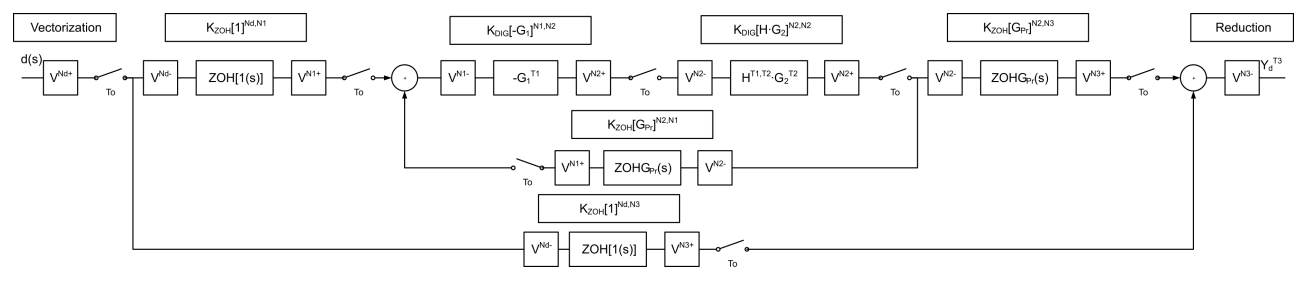

(f)

Figure 6.8: $\mathrm{Y} / \mathrm{d}$ MBDR lifted process 


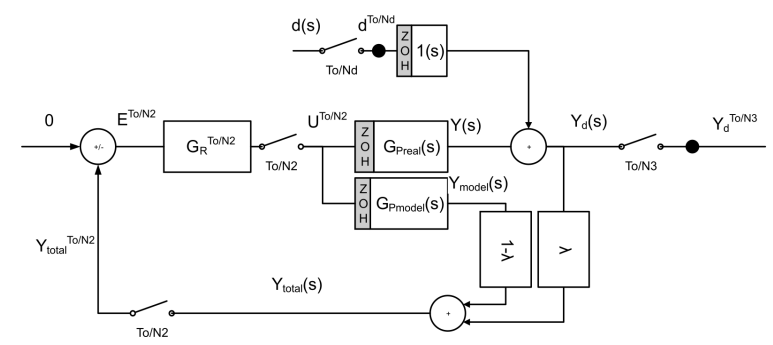

(a) Original diagram

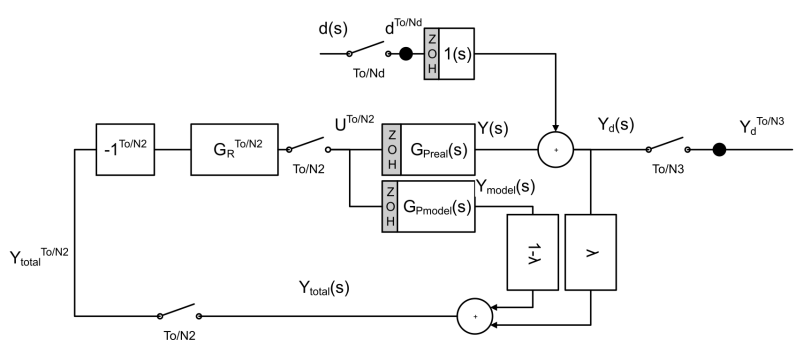

(b)

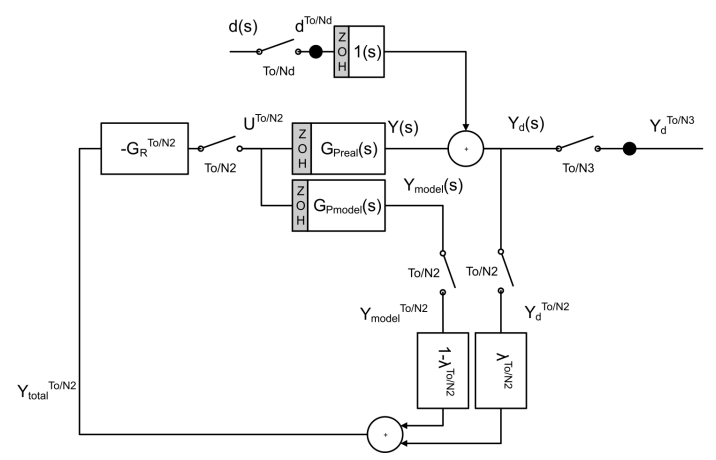

(c) 


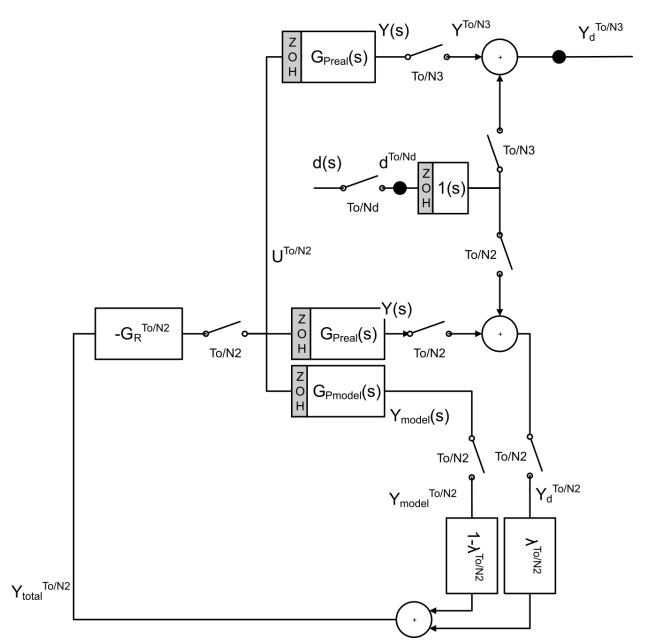

(d)

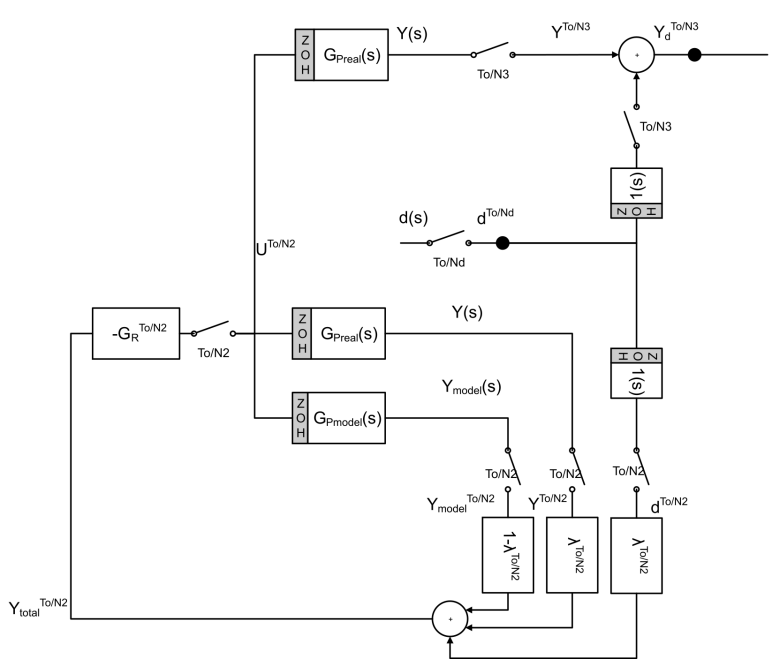

(e) 


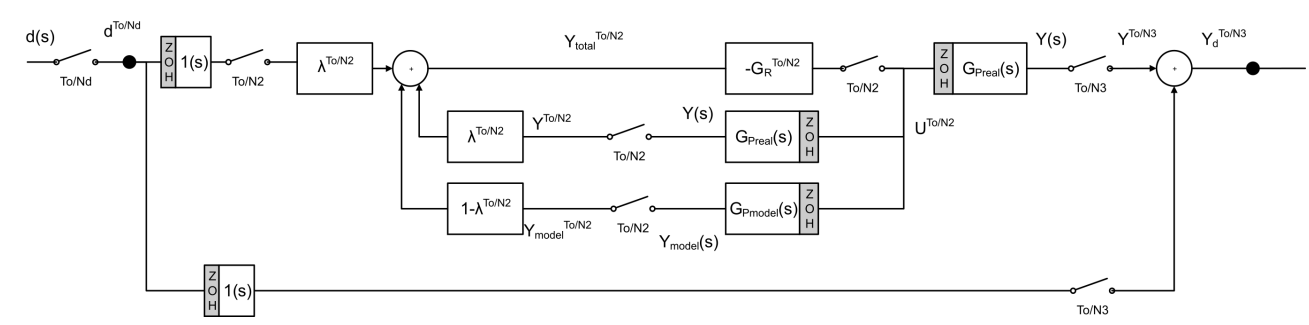

(f)

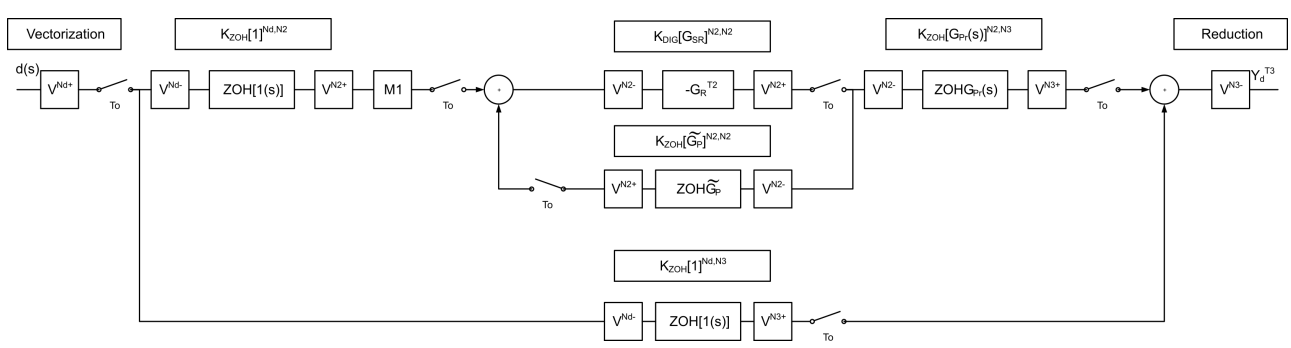

(g)

Figure 6.9: $\mathrm{Y} / \mathrm{d}$ IC lifted process

\subsection{Simulations}

In this section, the complete procedure is applied to two different simulations with different conclusions. The first step is to obtain the lifting modeling of MBDR and IC cases exposed in section 6.3 for each example. Then, the algebra introduced in section 6.5 and the DR systems FR introduced in section 6.4, allows the consideration of the QFT procedure in order to analyze both cases.

\subsubsection{Example 1}

The first is a continuous system with model:

$$
G_{\text {pmodel }}=\frac{1.5}{(s+0.5)(s+1.5)}
$$

this plant is controlled in a closed loop by a serial PID controller, 


$$
u(t)=K_{p}\left[e(t)+T_{D} \frac{d)}{d t} e(t)+\frac{1}{T_{i}} \int_{0}^{t} e(\tau) d \tau\right]
$$

with $K_{p}=8, T_{D}=0.2$, and $T_{i}=3.2$ in order to achieve some specifications. A discrete-time PID controller approximation for a sampling period $T$, is given by [53]:

$$
\begin{aligned}
& q_{0}=K_{p}\left(1+\frac{T_{D}}{T}\right) \\
& q_{1}=-K_{p}\left(1+2 \frac{T_{D}}{T}-\frac{T}{T_{i}}\right) \\
& q_{2}=K_{p} \frac{T_{D}}{T}
\end{aligned}
$$

Nevertheless, a multiplicative uncertainty has been assumed in the real system:

$$
G_{\text {preal }}=\frac{1.5}{(s+0.5)(s+1.5)} \frac{10 s+1}{100 s+1}
$$

that is, with a low frequency pole and high frequency zero. A single-rate control, which is called "fast" is designed for $T=0.1 \mathrm{~s}$. Due to diverse restrictions it is only possible to sample the output measurement every $T_{0}=0.3 \mathrm{~s}$. With these conditions the MBDR and IC with $N=3$ is planned.

Now the three options are going to be compared. In Figure 6.10, the closedloop step response is shown for the three controllers designed for the model plant (obviously the fast and inferential cases have the same response if ideal conditions are assumed). The application to the real process (that is, with model plant mismatching MPM) has been considered in Figure 6.11.

As it can be seen, the IC leads to a very slow output response, being the MBDR control response similar to the one of the fast single rate control. The closed-loop DR Bode diagram in Figure 6.12 confirms this behavior. As it can be seen, the IC case has a small bandwidth. The QFT analysis described in subsection 6.4 .3 with two reasonable restrictions for robust stability margins and output disturbance rejection $\mu=0.3$ and $\delta(w)=w / 1.5$ is shown in Figure 6.13 and Figure 6.14 for MBDR and IC cases respectively. A small gain increase in MBDR leads to the correct performance, but for the IC case even a high gain increment is not able to ensure the disturbance rejection for small 


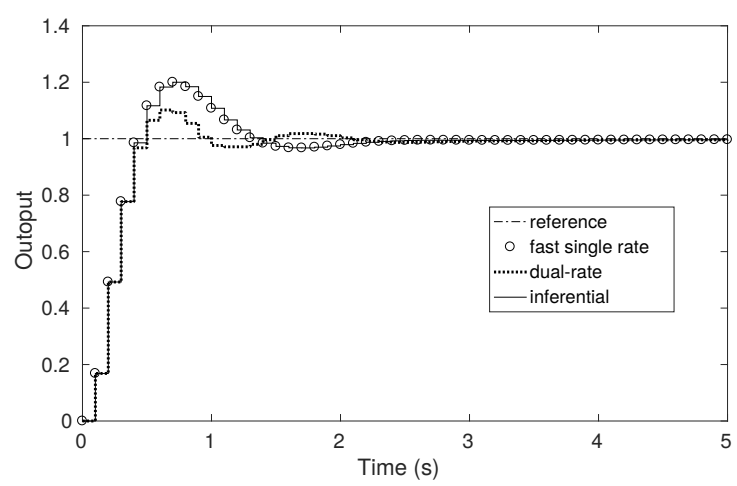

Figure 6.10: Example 1. Time response without MPM

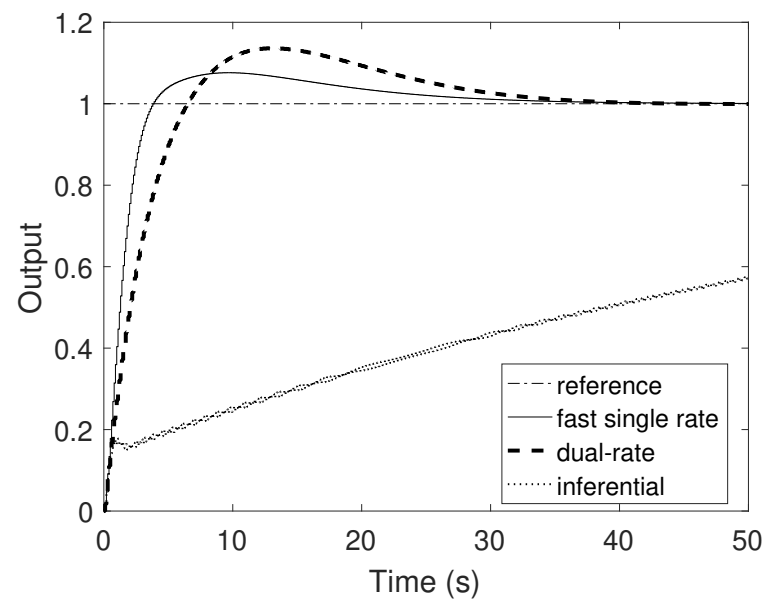

Figure 6.11: Example 1. Time response with MPM

frequencies and makes the system unstable. In Figure 6.14 the dashed line corresponds to the open loop shape incremented by a gain of 300. Following [54] the system is clearly unstable. The analysis has been performed with the help of the QFT Toolbox [55] with scripts adapted to the current problems.

Moreover, if the closed output response is analysed with respect to a step in the reference and an output disturbance $d(t)=\sin (0.04 t)$, (see Figure 6.15), the ineffective disturbance rejection capability of the IC is clear, while the MBDR control is valid for this purpose. 

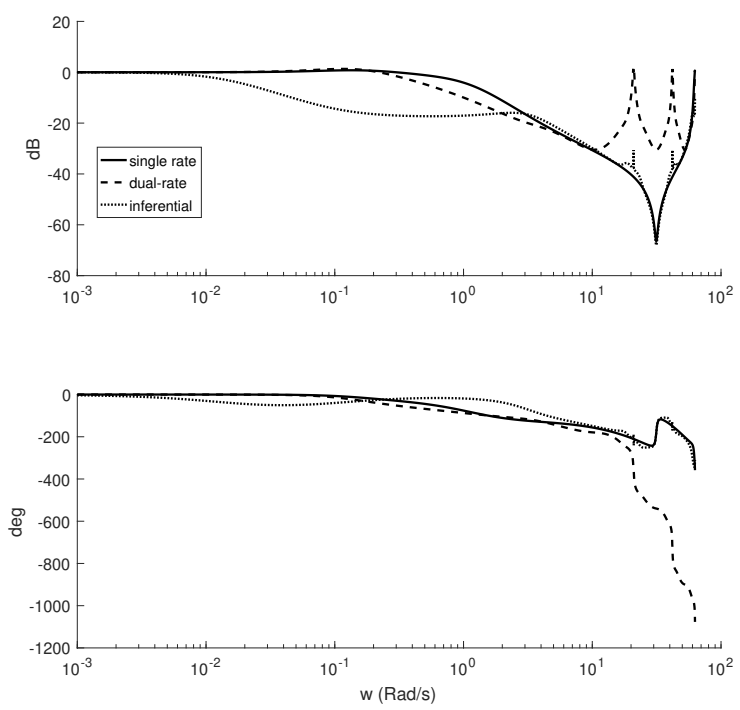

Figure 6.12: Example 1. Y/R DR systems Bode. Methods comparison

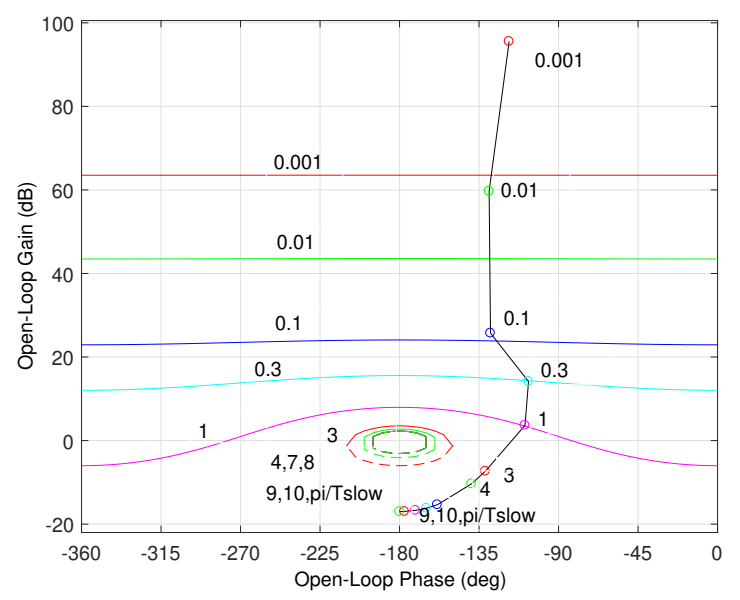

Figure 6.13: Example 1. MBDR case QFT analysis 


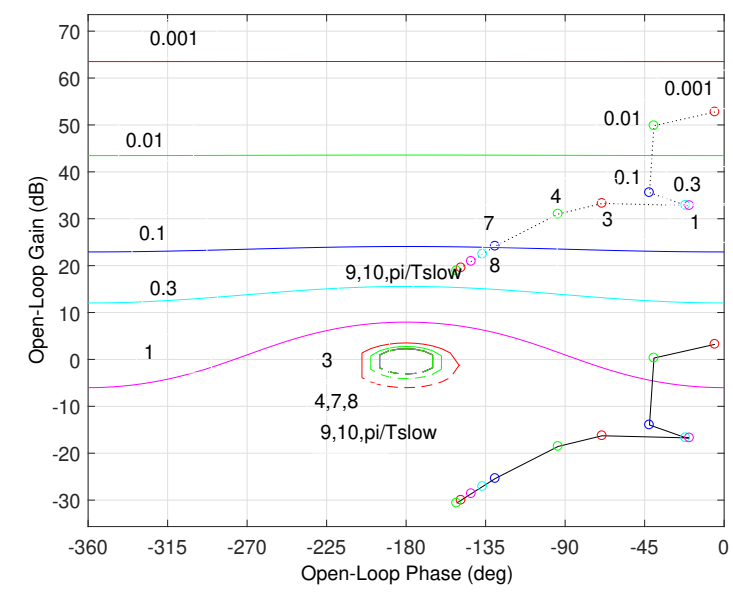

Figure 6.14: Example 1. IC case QFT analysis
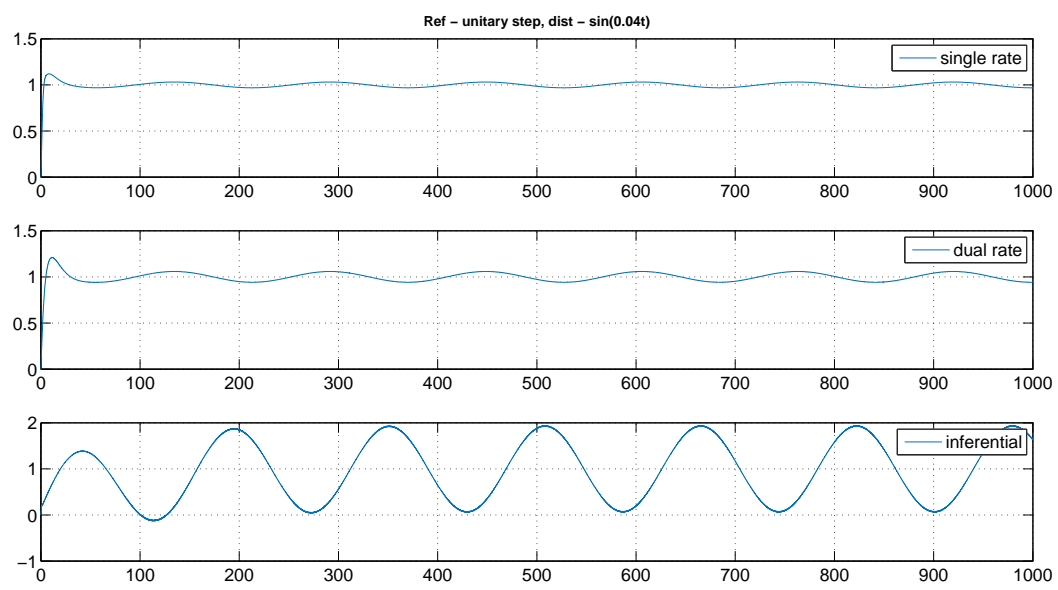

Figure 6.15: Disturbance rejection comparison

\subsubsection{Example 2}

The second example is taken from [34]. In this case, the discrete model process ${ }^{7}$ and the PI controller designed are

\footnotetext{
${ }^{7}$ it is possible to consider $T=1$. This is not a relevant fact for the current purposes
} 


$$
G_{\text {pmodel }}(z)=\frac{0.15}{z-0.9}, \quad G_{R}^{T}(z)=3+\frac{0.5}{1-z^{-1}}
$$

The actual plant is:

$$
G_{\text {preal }}=\frac{0.13}{z^{3}(z-0.92)}
$$

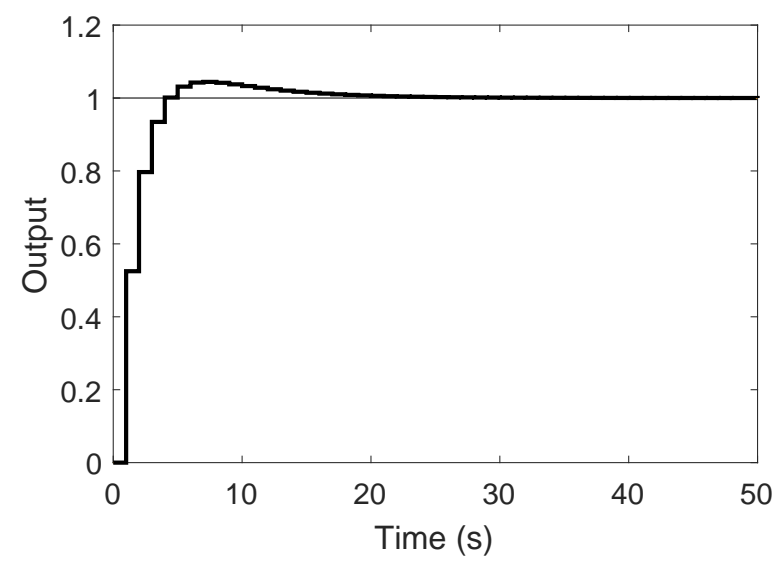

Figure 6.16: Example 2. Time response without MPM

Now a similar reasoning as in the example before is run. The closed loop step responses for the three controllers designed for the model plant are shown in Figure 6.16. In this case, the three closed-loops have a very similar output response. For this example the MBDR design has been adapted from [34]. Now the continuous closed-loop transfer function $M(s)$ is not known. Only $M^{T}(z)$ at $T$ is known. A simple discrete to continuous procedure was assumed for obtaining the discrete $N T$ transfer function $M^{N T}\left(z^{N}\right)$. As in [34] $N=4$ was considered. The application to the process with MPM is shown in Figure 6.17. In this case, the IC leads to the best closed loop output response (as it is said in [34]), being the single control unstable. The closed loop Bode diagrams are shown in Figure 6.18 and confirm this behavior. With the same tool used in example 1, the QFT analysis explains that in this case the IC is able to perform within the restrictions for stability $\mu=0.3$ and output disturbance rejection $\delta(w)=w / 1.5$ such it is depicted in Figure 6.19. In the MBDR case both restrictions are not satisfied. A slight increment of gain (about 1.3) would 


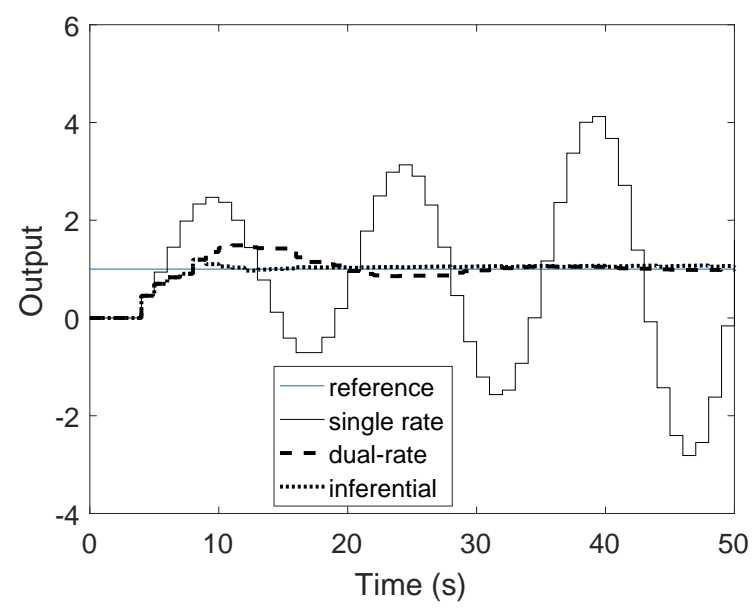

Figure 6.17: Example 2. Time response with MPM
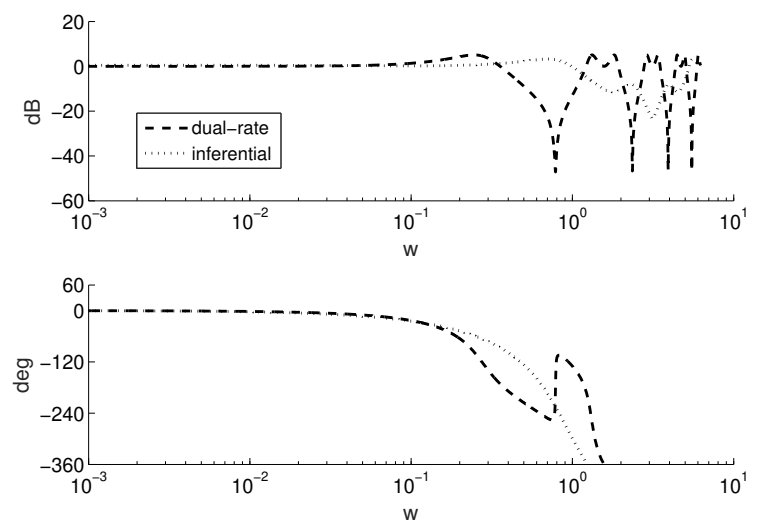

Figure 6.18: Example 2. Y/R DR systems Bode. Methods comparison

provide the output disturbance rejection compliance but the system would be unstable $(w=1.7,2 \mathrm{R} / \mathrm{s})$ as indicated by Figure 6.20 .

However, if the real plant were:

$$
G_{\text {preal }}=\frac{0.4}{z^{3}(z-0.96)}
$$




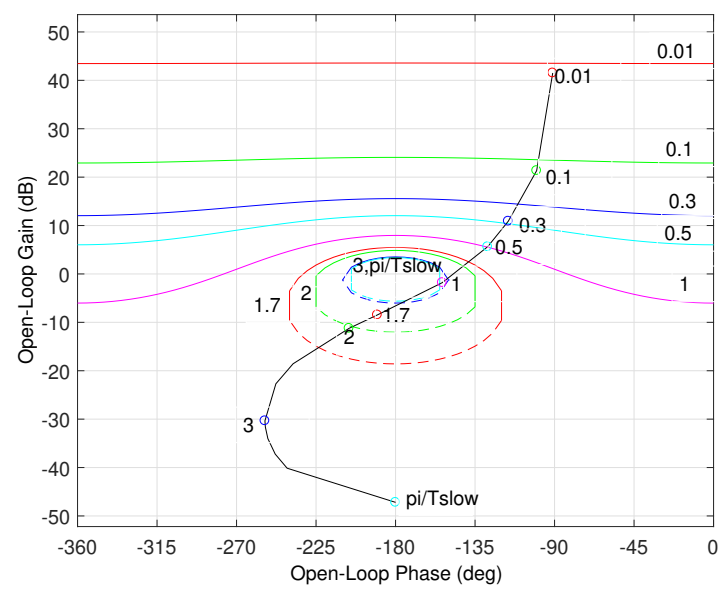

Figure 6.19: Example 2. IC case QFT analysis

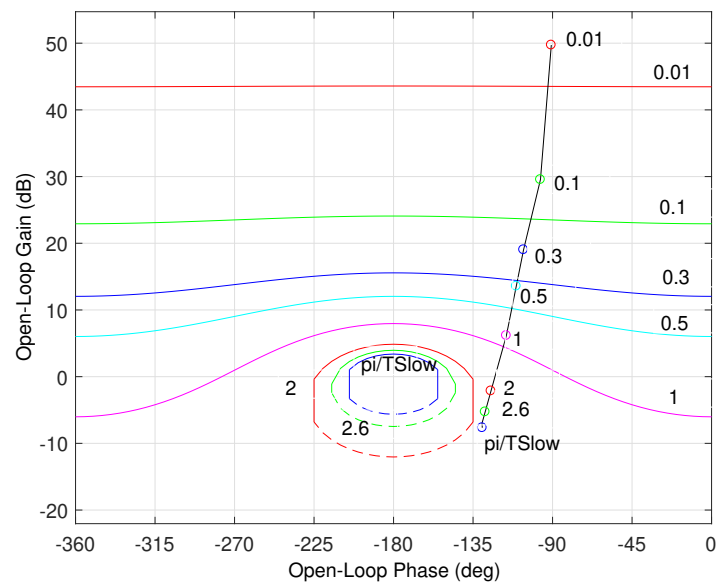

Figure 6.20: Example 2. MBDR case QFT analysis

that is, with the same transport delay but with different dynamics than the model, the IC would lead to instability and the MBDR would perform an acceptable system output response.

In general when there are significant differences in gain and/or constant times, the MBDR alternative is much better than the IC option. 


\subsection{Experimental Application}

At this point, the contents and procedures discussed before will be applied to a differential mobile robot path-tracking. The robot housing is formed by three plastic pieces constructed with a 3D printer. An Arduino Due with a 32-bit Atmel SAM3X8E ARM Cortex-M3 microcontroller was used. The robot has two wheels that are operated by two motors Pololu MG37D (12 volts) with encoders and two caster balls. A lithium battery with three cells and nominal voltage of 11.1 volts was utilized. An HC-SR04 ultrasonic sensor was used for measuring distances. In Figure 6.21 two pictures of the robot are shown.
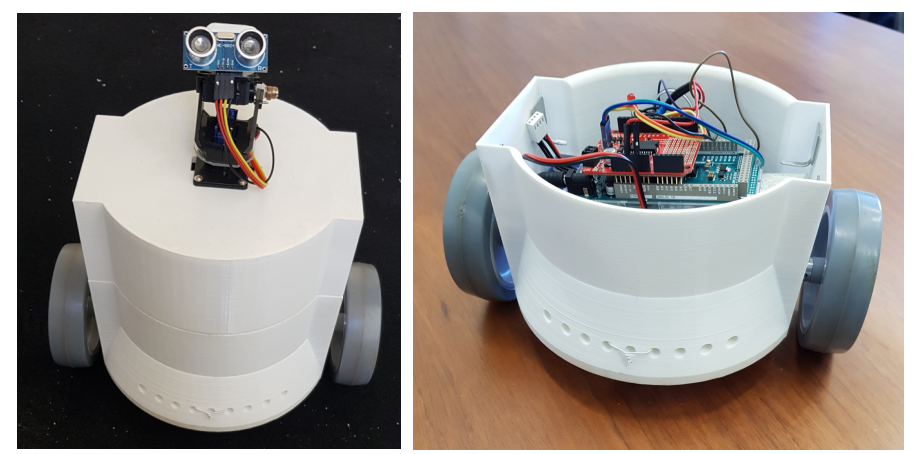

Figure 6.21: Autonomous robot used

This unmanned ground vehicle (UGV) should follow a predefined path. A pure pursuit tracking algorithm was considered. The position of the mobile robot is obtained by means of a energy consuming sensor and therefore restricts the time of autonomous use. It is proposed that this sensor could work less frequently but it is unfeasible in a classical single rate control due to the servomotors dynamics. In other words, a large sensing period but a short control update is needed in this scenario. Therefore, the problem introduced in this work reflects this situation. Both options, MBDR and IC, can be assumed. In both cases a $N$-slow measurement and fast control can be considered as previously described. 


\subsubsection{Path tracking}

As it was said the mobile robot must track a prescribed path. The first problem is to know in advance what is the next point to reach, that is the target point. There are different methods: follow the carrot, vector pursuit, pure pursuit and so on. In this work, a pure pursuit algorithm was used. Basically, the method tries to compute one arc between the current position and the target point of the predefined trajectory. For this purpose it is necessary to choose the next point to reach. It is usual to fix what is known as a look ahead distance so that the algorithm finds it. In this application, the pure pursuit method was selected because the path followed by the robot is smoother. The pure pursuit method and concepts like look ahead distance can be consulted in known references $[56,57]$. Once the future point has been computed, the differential mobile robot kinematics lead to the proper angular velocity of both wheels in order to get the target point, [58]. In our case, the linear speed was prefixed in a constant value that makes reaching the next point possible. Therefore, at every period the path tracking algorithm will provide the angular speed reference for each wheel once the current position is detected. A closed-loop for each of the wheels was considered assuming a slow measurement of actuators encoders. It would have been possible to use the encoders measurements to determine the robot position by odometry but the precision is much better with the selected procedure.

\subsubsection{Dual-rate controller}

As it was described, two options were considered: IC and MBDR. Both of them are planned assuming a slow measurement, that is, less sensor measures implies less energy consumption and therefore a greater battery operative life. This slow frequency can be processed as samples losses with respect to a fast frequency as it was already explained. In the IC case, the samples that have not been sensed are provided by an approximate actuator model while in MBDR case only the measurements obtained are used by the control algorithm.

In the set-up used, both servomotors (actuators) are considered to have the same transfer function (actually there are very slight differences).

$$
G_{p}(s)=\frac{W(s)}{V(s)}=\frac{0.1276}{0.1235 s+1}
$$

where the output $W$ is in $\mathrm{rad} / \mathrm{s}$, and the input $V$ in volts. The ideal control in order to achieve some specifications leads to a classical PI controller: 


$$
u(t)=6\left[e(t)+\frac{1}{0.12} \int_{0}^{t} e(\tau) d \tau\right]
$$

If it is discretized [53], the single-rate controller for $T=100 \mathrm{~ms}$ is:

$$
G_{r}^{T}(z)=\frac{6 z-1}{z-1}
$$

It was considered that the measurement should be $T_{0}=300 \mathrm{~ms}$, that is $N=3$ for a proper energy saving. With these conditions, the slow and fast controllers in the MBDR loop were:

$$
\begin{aligned}
G_{1}^{N T}\left(z^{N}\right) & =\frac{z_{s}^{2}-0.23 z_{s}+0.014}{z_{s}^{2}-1.08 z_{s}+0.08} \\
G_{2}^{T}(z) & =\frac{6.576 z^{2}-5.78 z+1.27}{z^{2}-0.976 z+0.24}
\end{aligned}
$$

where $z$ stands for $T=0.1$, and $z^{N}=z_{s}$ for $N T=3 T=0.3$. As it was explained, a digital hold is included between the slow and fast parts

$$
H^{N T, T}(z)=\frac{1-z^{-N}}{1-z^{-1}}
$$

In this case, in order to apply the considerations of this work, it is assumed that the model is:

$$
G_{m}(s)=\frac{0.2}{0.2 s+1}
$$

that is, there is a strong uncertainty in gain and time constant.

The QFT analysis, performed in a completely similar way than in section 6.6 with $\mu=0.6$ and $\delta(w) \leq w / 1.5$ for robust stability and output disturbance rejection, is depicted in Figure 6.22. Figure 6.23 shows the real trajectory followed by the UGV when the ideal, mismatch and mismatch with output disturbance are considered. As it can be seen, MBDR is much better than IC when the UGV must track an " $\mathrm{H}$ "-shaped path. The output disturbance considered was $d(t)=2 \sin (0.2 t)$. In regards to energy saving, when tracking 


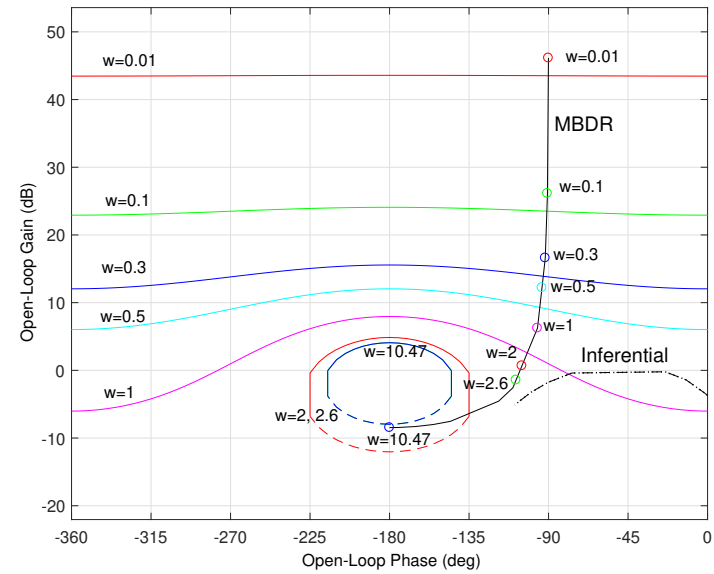

Figure 6.22: UGV QFT analysis
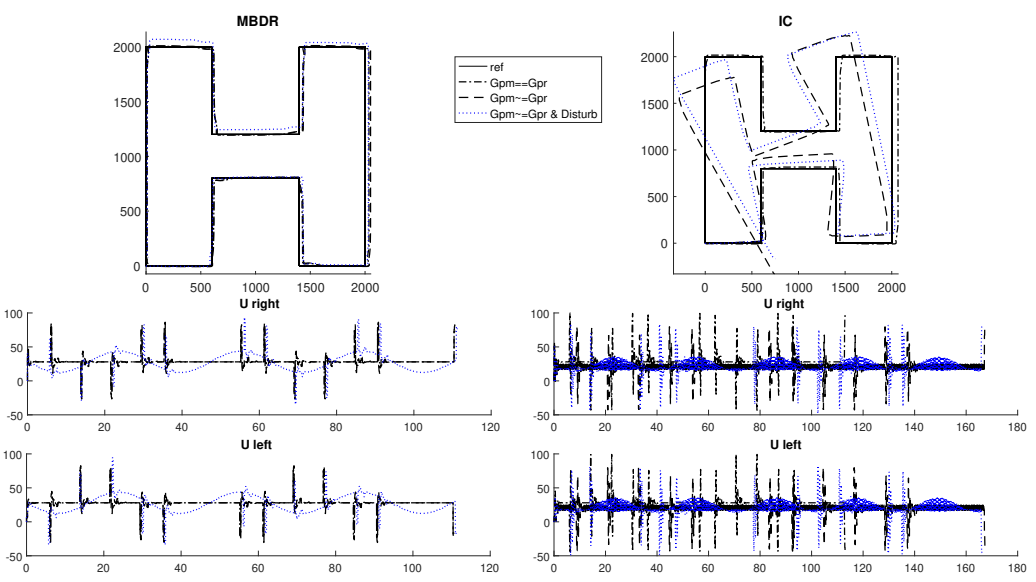

Figure 6.23: UGV Path tracking

the " $\mathrm{H}$ " reference, increasing the value of $N$ from 1 to 3 , the power consumption dropped by about $27 \%$ in our experiments. The power was calculated measuring intensity and voltage by means of a CompactRio (National Instruments) system. In order to analyze the influence of the non-linearities of the servomotors (dead zone and saturation) a simulation was carried out. The 
results showed in Figure 6.24 also confirm that the MBDR is better than the IC option.
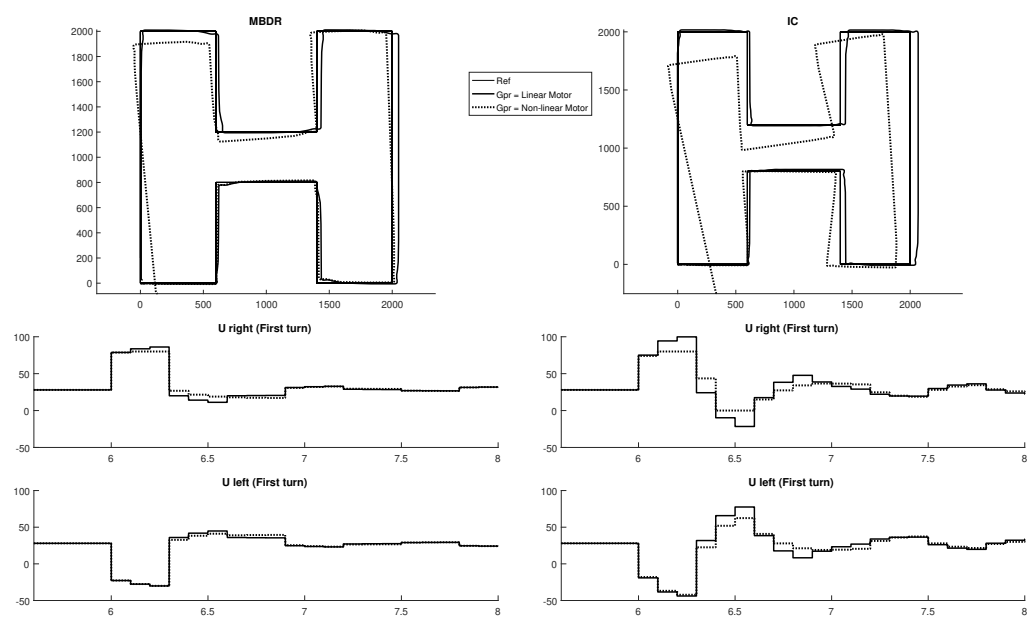

Figure 6.24: UGV. Linear and Non-linear servomotors comparison

\subsection{Conclusions}

In this contribution different DR model-based control design methodologies have been compared. These methods are motivated by restrictions that prevent the consideration of an ideal sampling pattern. The use of DR techniques allows to model this kind of situations, and a new efficient tool for DR FR computation is crucial for analyzing and detecting advantages or disadvantages for these different alternatives. It is very important how high the uncertainty of the real process is, that is, the comparison is problem-dependent but it seems that in any case the inferential control is unable to reject disturbances. A new QFT analysis procedure for DR systems has been assumed. Even though every case needs a particular analysis, when the model contains important uncertainties, the MBDR is a better option. Avenues for further research like the extension of this method to non-linear plants and the consideration of less simplistic inferential control strategies must be considered in the future. 


\section{Acknowledgements}

This work is funded in part by grant RTI2018-096590-B-100 from Spanish government, and by European Commission as part of Project H2020-SEC2016-2017 - Topic: SEC-20-BES-2016 - Id: 740736 - "C2 Advanced Multidomain Environment and Live Observation Technologies" (CAMELOT). Part WP5 supported by Tekever ASDS, Thales Research and Technology, Viasat Antenna Systems, Universitat Politècnica de València, Fundação da Faculdade de Ciências da Universidade de Lisboa, Ministério da Defensa Nacional - Marinha Portuguesa, Ministério da Administração Interna Guarda Nacional Republicana. 



\section{References}

[1] M.T. Guilandoust, A.J. Morris, and M.T. Tham. "Estimation and Control of Distillation Product Composition using Tray Temperature Measurements". In: IFAC Proceedings Volumes 19.15 (1986). IFAC Symposium on Dynamics and Control of Chemical Reactors and Distillation Columns, Bournemouth, UK, 8-10 December, 1986, pp. 203-208. ISSN: 1474-6670. DOI: https ://doi .org/10.1016/S1474-6670(17) 59422-7 (cit. on pp. 156, 157).

[2] D. Li et al. "Application of dual-rate modeling to CCR octane quality inferential control". In: IEEE Transactions on Control Systems Technology 11.1 (Jan. 2003), pp. 43-51. ISSN: 1063-6536. DOI: 10.1109/TCST . 2002.806433 (cit. on p. 156).

[3] J. A. Rossiter et al. "Robustness of dual-rate inferential MPC systems". In: Proceedings of the 2004 American Control Conference. Vol. 1. June 2004, pp. 251-253. DOI: 10.23919/ACC. 2004.1383613 (cit. on p. 156).

[4] Y. Fu et al. "Dual-rate adaptive decoupling controller and its application to a dual-tank water system". In: 2018 Annual American Control Conference (ACC). June 2018, pp. 2533-2538. DOI: 10.23919/ACC. 2018. 8431305 (cit. on p. 156).

[5] Luigi Fortuna et al. Soft Sensors for Monitoring and Control of Industrial Processes. Jan. 2007. ISBN: 978-1-84628-479-3. DOI: 10 . 1007/978-1 84628-480-9 (cit. on p. 156).

[6] Yuri Shardt and Biao Huang. "Tuning a Soft Sensor's Bias Update Term. 1. The Open-Loop CaseCase". In: Industrial \&5 Engineering Chemistry Research 51 (Mar. 2012), pp. 4958-4967. DOI: 10.1021/ie201456z (cit. on pp. 156, 157).

[7] Shima Khatibisepehr, Biao Huang, and Swanand Khare. "Design of inferential sensors in the process industry: A review of Bayesian methods". In: Journal of Process Control 23.10 (2013), pp. 1575-1596. ISSN: 0959-1524. 
DOI: https ://doi.org/10.1016/j.jprocont.2013.05.007 (cit. on p. 156).

[8] Shima Khatibisepehr and Biao Huang. "Dealing with Irregular Data in Soft Sensors: Bayesian Method and Comparative Study". In: Industrial 6 Engineering Chemistry Research 47.22 (2008), pp. 8713-8723. DOI: 10.1021/ie800386v (cit. on p. 156).

[9] Torsten Söderström. "On some adaptive controllers for stochastic systems with slow output sampling". In: Methods and applications in adaptive control. Springer, 1980, pp. 64-73 (cit. on p. 156).

[10] Lennart Ljung. "Analysis of recursive stochastic algorithms". In: IEEE transactions on automatic control 22.4 (1977), pp. 551-575 (cit. on p. 157).

[11] William L Luyben. "Parallel cascade control". In: Industrial \& Engineering Chemistry Fundamentals 12.4 (1973), pp. 463-467 (cit. on p. 157).

[12] Nandkishor G Patke, Pradeep B Deshpande, and Adam C Chou. "Evaluation of inferential and parallel cascade schemes for distillation control". In: Industrial \&3 Engineering Chemistry Process Design and Development 21.2 (1982), pp. 266-272 (cit. on p. 157).

[13] Mohammad T Guilandoust, A Julian Morris, and Ming T Tham. "An adaptive estimation algorithm for inferential control". In: Industrial $\& 3$ engineering chemistry research 27.9 (1988), pp. 1658-1664 (cit. on p. 157).

[14] Yuri AW Shardt, Haiyang Hao, and Steven X Ding. "A new soft-sensorbased process monitoring scheme incorporating infrequent KPI measurements". In: IEEE Transactions on Industrial Electronics 62.6 (2015), pp. 3843-3851 (cit. on p. 157).

[15] CB Brosilow and M Tong. "The structure and dynamic of inferential control system". In: AIChE Journal 24 (1978), pp. 492-499 (cit. on p. 157).

[16] Jay H Lee and Manfred Morari. "Robust inferential control of multi-rate sampled-data systems". In: Chemical Engineering Science 47.4 (1992), pp. 865-885 (cit. on p. 157).

[17] Yuri AW Shardt and Biao Huang. "Tuning a Soft Sensor's Bias Update Term. 2. The Closed-Loop Case". In: Industrial \& Engineering Chemistry Research 51.13 (2012), pp. 4968-4981 (cit. on p. 157).

[18] F Morant et al. "Adaptive Control of a Cement Raw-Material Study of Different Control Algorithms/Supervisory Level". In: IFAC Proceedings Volumes 21.7 (1988), pp. 129-134 (cit. on p. 157).

[19] F Morant et al. "RIGAS: An intelligent controller for cement kiln control". In: Proc. IFAC Symp. Artif. Intelligence in Real Time Control. 1992 (cit. on p. 157). 
[20] Chang Chieh Hang, Khiang Wee Lim, and BW Chong. "A dual-rate adaptive digital Smith predictor". In: Automatica 25.1 (1989), pp. 1-16 (cit. on p. 157).

[21] Anthony M Phillips and Masayoshi Tomizuka. "Multirate estimation and control under time-varying data sampling with applications to information storage devices". In: Proceedings of 1995 American Control Conference-ACC'95. Vol. 6. IEEE. 1995, pp. 4151-4155 (cit. on p. 157).

[22] C Cosner, G Anwar, and M Tomizuka. "Plug in repetitive control for industrial robotic manipulators". In: Proceedings., IEEE International Conference on Robotics and Automation. IEEE. 1990, pp. 1970-1975 (cit. on p. 157).

[23] Pedro Albertos, Julian Salt, and Josep Tornero. "Dual-rate adaptive control". In: Automatica 32.7 (1996), pp. 1027-1030 (cit. on p. 157).

[24] Weiping Lu and G Fisher. "Multirate adaptive inferential estimation". In: IEE Proceedings D (Control Theory and Applications). Vol. 139. 2. IET. 1992, pp. 181-189 (cit. on p. 157).

[25] Dongguang Li, Sirish L Shah, and Tongwen Chen. "Identification of fastrate models from multirate data". In: International Journal of Control 74.7 (2001), pp. 680-689 (cit. on p. 157).

[26] Majid Koul, M Manivannan, and SK Saha. "Effect of Dual-rate Sampling on the Stability of a Haptic Interface". In: Journal of Intelligent 63 Robotic Systems 91.3-4 (2018), pp. 479-491 (cit. on p. 157).

[27] Julián Salt and Masayoshi Tomizuka. "Hard disk drive control by model based dual-rate controller. Computation saving by interlacing". In: Mechatronics 24.6 (2014), pp. 691-700 (cit. on p. 157).

[28] Raul Mansano, Eduardo Godoy, and Arthur Porto. "The benefits of soft sensor and multi-rate control for the implementation of wireless networked control systems". In: Sensors 14.12 (2014), pp. 24441-24461 (cit. on p. 157).

[29] Ángel Cuenca et al. "A Delay-Dependent Dual-Rate PID Controller Over an Ethernet Network". In: IEEE Transactions on Industrial Informatics 7.1 (Feb. 2011), pp. 18-29. ISSN: 1551-3203. DOI: 10.1109/TII . 2010. 2085007 (cit. on p. 157).

[30] Tian You Chai et al. "Dual Rate Adaptive Control for an Industrial Heat Supply Process Using Signal Compensation Approach". In: IFACPapersOnLine 50.1 (2017), pp. 1877-1884 (cit. on p. 157). 
[31] Á. Cuenca et al. "Periodic Event-Triggered Sampling and Dual-Rate Control for a Wireless Networked Control System With Applications to UAVs". In: IEEE Transactions on Industrial Electronics 66.4 (Apr. 2019), pp. 3157-3166. ISSN: 0278-0046. DOI: 10.1109/TIE.2018.2850018 (cit. on p. 157).

[32] Mitsuhiro Nakayama, Hiroshi Oku, and Shun Ushida. "An Experiment on Closed-loop System Identification of UAV Using Dual-rate Sampling". In: IFAC-PapersOnLine 51.15 (2018), pp. 598-603 (cit. on p. 157).

[33] Ángel. Cuenca et al. "A packet-based dual-rate PID control strategy for a slow-rate sensing Networked Control System". In: ISA Transactions 76 (2018), pp. 155-166. ISSN: 0019-0578. DOI: https://doi.org/10.1016/ j.isatra.2018.02.022 (cit. on p. 157).

[34] Dongguang Li, Sirish L Shah, and Tongwen Chen. "Analysis of dual-rate inferential control systems". In: Automatica 38.6 (2002), pp. 1053-1059 (cit. on pp. 157, 184, 185).

[35] Julián Salt and Pedro Albertos. "Model-based multirate controllers design". In: IEEE Transactions on Control Systems Technology 13.6 (Nov. 2005), pp. 988-997. ISSN: 1063-6536. DOI: 10.1109/TCST . 2005.857410 (cit. on pp. 158, 160, 161).

[36] Isaac Horowitz. "Quantitative feedback theory". In: IEE Proceedings DControl Theory and Applications. Vol. 129. 6. IET. 1982, pp. 215-226 (cit. on pp. 158, 167, 170).

[37] Oded Yaniv. Quantitative feedback design of linear and nonlinear control systems. Springer Science \& Business Media, 1999 (cit. on p. 158).

[38] Wen Hua Chen and Donald J Ballance. "QFT design for uncertain nonminimum phase and unstable plants revisited". In: International journal of control 74.9 (2001), pp. 957-965 (cit. on p. 158).

[39] MA Franchek, P Herman, and ODI Nwokah. "Robust nondiagonal controller design for uncertain multivariable regulating systems". In: Journal of dynamic systems, measurement, and control 119.1 (1997), pp. 80-85 (cit. on p. 158).

[40] Alfonso Baños and Antonio Barreiro. "Stability of non-linear QFT designs based on robust absolute stability criteria". In: International Journal of Control 73.1 (2000), pp. 74-88 (cit. on p. 158).

[41] J. Salt and A. Sala. "A new algorithm for dual-rate systems frequency response computation in discrete control systems". In: Applied Mathematical Modelling 38.23 (2014), pp. 5692-5704 (cit. on pp. 159, 167, 168). 
[42] Julián Salt et al. "A Multirate Control Strategy to the Slow Sensors Problem: An Interactive Simulation Tool for Controller Assisted Design". In: Sensors 14.3 (2014), pp. 4086-4110. ISSN: 1424-8220. DOI: 10.3390/ s140304086 (cit. on p. 160).

[43] G. Kranc. "Input-output analysis of multirate feedback systems". In: $A u$ tomatic Control, IRE Transactions on 3.1 (1957), pp. 21-28 (cit. on p. 162).

[44] B. Bamieh et al. "A lifting technique for linear periodic systems with applications to sampled-data control". In: Systems \& Control Letters 17.2 (1991), pp. 79-88 (cit. on pp. 162, 164).

[45] P. Khargonekar, K. Poolla, and A. Tannenbaum. "Robust control of linear time-invariant plants using periodic compensation". In: IEEE Transactions on Automatic Control 30.11 (Nov. 1985), pp. 1088-1096. ISSN: 0018-9286. DOI: 10.1109/TAC.1985.1103841 (cit. on pp. 162, 165).

[46] B.A. Francis and T.T. Georgiou. "Stability theory for linear time-invariant plants with periodic digital controllers". In: Automatic Control, IEEE Transactions on 33.9 (1988), pp. 820-832 (cit. on pp. 162, 165).

[47] Richard F Whitbeck and DCJ Didaleusky. "Multirate digital control systems in simulation applications". In: Report AFWAL-TR-80-3101, Vols. I, II and III, 1980 (1980) (cit. on p. 163).

[48] DAVID G Meyer. "Shift-invariant equivalents for a new class of shiftvarying operators with applications to multi-rate digital control". In: Decision and Control, 1988., Proceedings of the 27th IEEE Conference on. IEEE. 1988, pp. 1697-1701 (cit. on p. 165).

[49] P. M. Thompson. "Gain and phase margins of multi-rate sampled-data feedback systems". In: International Journal of control 44.3 (1986), pp. 833846 (cit. on pp. 165, 166).

[50] Isaac M Horowitz. Synthesis of feedback systems. Elsevier, 2013 (cit. on pp. 167, 170).

[51] Julián Salt and José Alcaina. "Dual-rate sampled-data systems. Some interesting consequences from its frequency response analysis". In: International Journal of General Systems 48.5 (2019), pp. 554-574. DOI: https://doi.org/10.1080/03081079.2019.1608984 (cit. on p. 168).

[52] Oded Yaniv and Yossi Chait. "Direct control design in sampled-data uncertain systems". In: Automatica 29.2 (1993), pp. 365-372 (cit. on p. 170).

[53] R Iserman. Digital Control. 1990 (cit. on pp. 181, 190). 
[54] N Cohen et al. "Stability Analysis using Nichols Charts". In: International Journal of Robust and Nonlinear Control 4.1 (1994), pp. 21-46 (cit. on p. 182).

[55] Craig Borghesani, Yossi Chait, and Oded Yaniv. The QFT Frequency Domain Control Design Toolbox for use with Matlab. Citeseer, 1993 (cit. on p. 182).

[56] Martin Lundgren. "Path tracking and obstacle avoidance for a miniature robot". In: Umeå University, Umeå, Master Thesis (2003) (cit. on p. 189).

[57] Moveh Samuel, Mohamed Hussein, and Maziah Binti Mohamad. "A review of some pure-pursuit based path tracking techniques for control of autonomous vehicle". In: International Journal of Computer Applications 135.1 (2016), pp. 35-38 (cit. on p. 189).

[58] Gregory Dudek and Michael Jenkin. Computational principles of mobile robotics. Cambridge university press, 2010 (cit. on p. 189). 


\section{Chapter 7}

\section{Paper 6. Energy-efficient control for an un- manned ground vehicle in a wireless sensor network}

José Alcaina, Ángel Cuenca, Julián Salt, Minghui Zheng, Masayoshi Tomizuka

In this paper, an energy-efficient control solution for an Unmanned Ground Vehicle (UGV) in a Wireless Sensor Network is proposed. This novel control approach integrates periodic event-triggered control, packet-based control, time-varying dual-rate Kalman filter based prediction techniques, and dual-rate control. The systematic combination of these control techniques allows the UGV to track the desired path preserving performance properties, despite: i) existing scarce data due to the reduced usage of the wireless sensor device, which results in less number of transmissions through the network, and hence, bandwidth and battery saving, ii) appearing some wireless communication problems such as time-varying delays, packet dropouts and packet disorder, and iii) coping with a realistic scenario where external disturbance and sensor noise can arise. The main benefits of the control solution are illustrated via simulation.

Keywords: Wireless sensor network; Energy efficiency; Kalman filter; dual-rate control; event-triggered control; packet-based control.

Journal of Sensors, volume 2019, oct 2019, ID 7085915, 16 pages, https://doi.org/10.1155/2019/7085915 


\subsection{Introduction}

A Wireless Sensor Network (WSN) [1, 2] can be defined as a group of sensors for monitoring and recording system conditions, which are wirelessly transferred to a central location. In recent years, the development of smart sensors has led WSNs to gaining worldwide attention. Some trending applications on WSNs are target tracking [3, 4], health monitoring [5, 6], and environment exploration [7, 8]. Smart sensor nodes are low power devices equipped with one or more sensors, a digital processor, memory, a power module, a wireless communication module, and an actuator. Sensors of different nature (mechanical, optical, magnetic, etc) may be attached to the sensor node to measure system variables. Since memory of the sensor nodes is limited, the wireless communication enables to transfer the measured data to a base station (e.g., a laptop), which is usually provided with powerful processing and storage capabilities. In this way, the base station may be able to compute complex control algorithms.

Differently from traditional networks, a WSN has its own design and resource constraints. Resource constraints include short communication range, a limited amount of energy (which is supplied by battery), low bandwidth, and limited processing and storage capabilities (being not capable to run complex control algorithms). As sensor nodes operate on limited battery power, and data transmission is very expensive in terms of energy consumption [9], a central aspect in WSNs is the optimization of communication in order to reduce energy usage. In other words, one of the main aims in WSNs is to get efficient reliable wireless communications in order to maximize network lifetime. This aspect has been widely treated in literature therein from different points of view. In general, three main techniques are typically used [10]: duty cycling, data-driven approaches, and mobility. Duty cycling allows nodes to assume a low-power, idle state whenever they are not transmitting or receiving (see, e.g., in $[11,12])$. Data-driven approaches are designed to reduce the amount of sampled and transmitted data (see, e.g., [13, 14]). Mobility is focused on minimizing the communication distance and altering traffic flow (see, e.g., $[15,16])$. In the present paper, a novel data-driven approach based on the systematic combination of periodic event-triggered control, packet-based control, time-varying dual-rate Kalman filter based prediction techniques, and dual-rate control is proposed. As a data-driven approach, the control solution enables to reduce the sensor node usage, which implies less packet deliveries, and hence, battery and bandwidth saving. This reduction of the resource utilization can be reached while preserving performance properties when tracking a desired target. In addition, the control proposal is able to guarantee reliable 
packet delivery by dealing with some wireless communication problems such as network-induced delays, packet dropouts, and packet disorder. Finally, the control approach is also able to cope with realistic scenarios, where external disturbance and sensor noise can arise.

In Periodic Event-Triggered Control (PETC) [17, 18], system data are transferred only when output variables satisfy certain event conditions, which are periodically evaluated. Compared to the traditional time-triggered control, PETC enables to further reduce resource utilization such as bandwidth and energy consumption [19, 20]. As the controller (located at the base station) is provided with less system data, event-based state prediction techniques must be additionally included in order to estimate the not available data and keep performance properties. Both continuous-time (see, e.g., [17]) and discretetime frameworks (see, e.g., [21]) are found in literature on PETC. In this paper, the last case is adopted by integrating a time-varying dual-rate Kalman filter with a dual-rate controller.

Regarding event-based state prediction techniques, different approaches address this aspect in literature. For instance, distributed event-triggered filtering in $[13,14,22]$, model-based predictor in [18, 23], time-varying Kalman filters in [24-26]. Following this line of research on time-varying Kalman filters, in this paper a Time-Varying Dual-Rate Kalman Filter (TVDRKF) is used in order to provide not available data and compute an $h$-step ahead state prediction stage. The nature of this filter is: i) dual-rate, due to the consideration of a slow-rate state correction stage along with a fast-rate prediction stage; and ii) time-varying, because the correction stage is carried out in a non-uniform fashion as a consequence of the event-triggered sampling scheme. Unlike [26], the Kalman filter is now integrated in a WSN, where communication problems such as network-induced delays and packet dropouts can appear. Hence, the TVDRKF is able to estimate the not available data not only due to the event-triggering sampling but also due to the packet dropouts. In addition, the TVDRKF is also able to compensate for the time-varying network-induced delays. To cope with both problems, the integration of packet-based control strategies in the control solution is needed.

Packet-based control $[27,28]$ is a technique which enables to decrease the communication rate by simultaneously sending a set of data in each transmission. In our work, this strategy is not only useful for resource saving purposes but also for complementing the TVDRKF, since the controller (located at the base station, and including the TVDRKF) is allowed to send a set of $h$-step ahead control signal estimations in a packet through the network to the actuator (located at the sensor node). The computation of the control signal estima- 
tions is carried out from the set of $h$-step ahead state predictions obtained by the TVDRKF, and following a delay-free control algorithm. This is a relevant aspect of this work, since, for implementation purposes, the round-trip time delay is not required to be measured and compensated for, which makes the solution applicable to a wide range of WSN where the time delay is difficult to measure. In this way, at the actuator, when no packet arrives, estimated control actions received in the previous transmission are applied to the plant, not being influenced by the time-varying network-induced delay. When the packet arrives after the delay, the estimated control signal is replaced with the actual one. The difference between actual and estimated control signals should be negligible, since, i) an accurate system model is assumed, and ii) although a realistic scenario is considered, where possible external disturbance and measurement noise can arise, these aspects are faced by the TVDRKF. To the best of the authors' knowledge, the working mode of the proposed control algorithm is novel in this kind of frameworks.

In dual-rate control [29-31], a slower sensing rate in comparison to a faster actuation can be assumed. The event-triggered conditions at the sensor node are periodically evaluated at the slow rate. Despite sensing in this way, the fast actuation enables to achieve acceptable control properties. In addition, dual-rate control techniques provide twofold benefits: i) to lessen the amount of transmissions through the network, which results in energy and bandwidth saving, ii) to elude packet disorder, selecting the sampling period to be larger than the largest round-trip time delay. As a statistical distribution for the networkinduced delay is assumed to be known [32], the largest delay can be easily found. In the present work, due to the broad knowledge of PID controllers in academic and industrial environments, a dual-rate PID control scheme is considered. The estimated disturbance signal obtained by the TVDRKF is used to finally tune the PID control signal.

In order to show the potential of the ideas posed in this work, the control solution is employed in the context of a popular control application, that of Unmanned Ground Vehicles (UGVs). In fact, the large number of UGV applications such as target tracking, inspection, mapping, etc, has attracted attention of the scientific community (see, e.g, $[33,34]$ ). In our work, a target tracking application in a WSN is developed, where the Pure Pursuit path tracking algorithm $[35,36]$ is used in order to make the UGV follow a pre-defined path approximately. The algorithm computes velocity commands from velocity estimations provided by the TVDRKF. The UGV chosen will be a differential robot. 
In summary, the main contribution of the present work is the development of a novel and complete approach for WSNs, where a TVDRKF, a dual-rate controller and a Pure Pursuit path tracking algorithm are systematically brought together in a PETC scenario in order to considerably lessen resource usage (energy and bandwidth), and dealing with some wireless communication problems, and disturbance and noise, while keeping satisfactory control properties.

Finally, the work is structured as follows. section 7.2 presents the energyefficient control solution. section 7.3 introduces the event-triggered conditions and control structures used in the control solution. section 7.4 presents some cost indexes to analyze the trade-off between control performance and resource utilization. In section 7.5, a realistic application of the control solution to UGVs is simulated, and its effectiveness validated, via a simulation tool known as Truetime [37], which is based on Matlab/Simulink ${ }^{\circledR}$. section 7.6 summarizes the main conclusions of the paper.

\subsection{Problem scenario}

The energy-efficient control solution taken into account in this work is illustrated in Figure 7.1. The control system presents two components: the sensor node, where the sensor, actuator, and plant are included, and the base station, where the control algorithm is implemented. In both components, some trigger mechanisms are used to decide when the data is going to travel through the network. The wireless network connects both sides and introduces some communication problems such as time-varying delays, packet dropouts, and packet disorder. In the next subsections, these problems are formally described, and the working mode of the control structure is presented.

\subsubsection{Time-varying network-induced delays, packet dropouts, and packet disorder}

As said in the previous section, dual-rate control is used in the proposed approach. Then, two different periods are considered: $T$ as the actuation period, and $N T$ as the sensing period, being $N \in \mathbb{N}^{+}$the multiplicity between the two periods of the dual-rate control scheme [30]. Let us respectively denote $(.)_{k}^{T}$ and $(.)_{k}^{N T}$ as a $T$-period and an $N T$-period signal or variable, where $k \in \mathbb{N}$ are iterations at the corresponding period. For a packet sampled at instant $k N T$, being effectively sent (i.e., the trigger conditions hold) and not lost, the round-trip time delay is defined as 


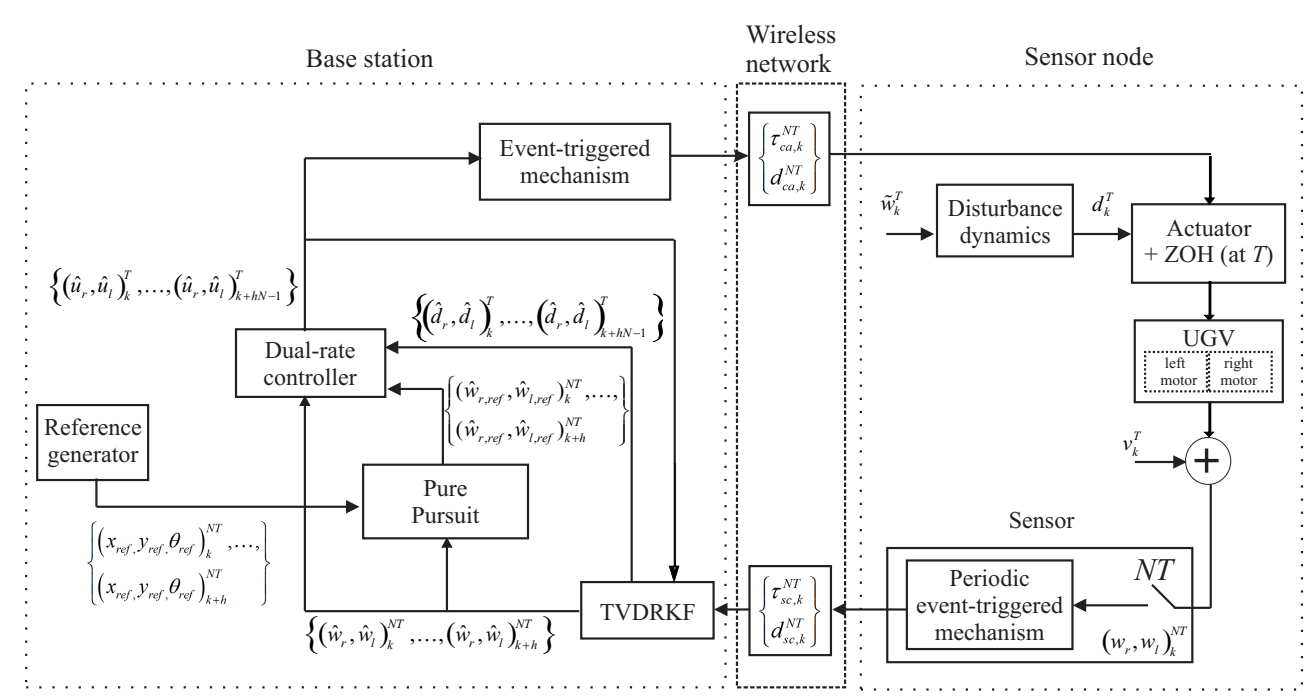

Figure 7.1: Energy-efficient control system for a WSN

$$
\tau_{k}^{N T}=\tau_{s c, k}^{N T}+\tau_{c a, k}^{N T}+\tau_{c, k}^{N T}
$$

being $\tau_{s c, k}^{N T}$ the sensor-to-controller network-induced delay, $\tau_{c a, k}^{N T}$ the controllerto-actuator delay, and $\tau_{c . k}^{N T}$ a computation time delay. As a local clock is assumed to govern the different devices located at the sensor node, they can be completely synchronized. As a consequence, in order to measure the roundtrip time delay, no additional time-stamping techniques and synchronization are needed. The delay can be measured by subtracting packet sending and receiving times. Note that, although a shared clock can be supposed in some WSN applications (as in this work), it might be hard to satisfy for other applications, for example, in distributed wireless sensor nodes, where an option may be to synchronize nodes [38].

To avoid packet disorder, in this work it is strictly necessary to know the maximum round-trip time delay $\tau_{\max }=\max \left(\tau_{k}^{N T}\right)$ such that $\tau_{\max }<N T$. This condition may be relaxed by taking advantage of the Kalman filter based estimation techniques used in this work, but additionally requiring the implementation of time-stamping techniques to detect the disorder. From off-line experiences on the WSN, the statistical distribution of the round-trip time delay can be obtained, and hence, $\tau_{\max }$. Since, in the present work, UDP is used as the transport layer protocol, the delay distribution may be approximated as 
a generalized exponential distribution [39]. The consequent probability density function can be expressed as follows

$$
P\left[\tau_{k}^{N T}\right]= \begin{cases}\frac{1}{\phi} e^{\frac{-\left(\tau_{k}^{N T}-\eta\right)}{\phi}} & , \quad \text { if } \tau_{k}^{N T} \geq \eta \\ 0 & , \quad \text { if } \tau_{k}^{N T}<\eta\end{cases}
$$

being the variance of the delay $V\left[\tau_{k}^{N T}\right]=\phi^{2}$, and its expected value $E\left[\tau_{k}^{N T}\right]=$ $\phi+\eta$. The median of the delay may be a viable choice for $\eta$, and $\phi$ can be deduced from $\eta$ and an experimental value of $E\left[\tau_{k}^{N T}\right]$.

As well-known, if UDP is used as the transport layer protocol, packet dropouts may appear. Since this phenomenon is basically random [32], it can be modeled as a Bernoulli distribution [40]. Let us respectively indicate the possible sensorto-controller and controller-to-actuator packet dropouts by means of $d_{s c, k}^{N T}$ and $d_{c a, k}^{N T}$. In the present work, both cases are contemplated as a Bernoulli process, whose probability of dropout is respectively given by $p_{s c}$ and $p_{c a}$ :

$$
\begin{aligned}
& p_{s c}=\operatorname{Pr}\left[d_{s c, k}^{N T}=0\right] \in[0,1) \\
& p_{c a}=\operatorname{Pr}\left[d_{c a, k}^{N T}=0\right] \in[0,1)
\end{aligned}
$$

\subsubsection{Control structure}

Next, the two components (sensor node and base station) and the signals involved in the energy-efficient control system are detailed.

As shown in Figure 7.1, the sensor node includes a UGV as the plant to be controlled, which is composed of two motors (for right and left wheels) and is subject to noise $v_{k}^{T}$ and disturbance $d_{k}^{T}$ signals. The control action to be applied to the right and left motors is respectively $\left(\hat{u}_{r}, \hat{u}_{l}\right)_{k}^{T}$. The output to be sensed is the rotational velocity of both motors $\left(w_{r}, w_{l}\right)_{k}^{N T}$. This output is sent to the base station when the periodic event-triggered condition defined at the sensor device holds.

The base station includes the control algorithm, which integrates a TimeVarying Dual-Rate Kalman Filter (TVDRKF), a Pure Pursuit path tracking algorithm (which receives the desired path from the reference generator), and a dual-rate controller. In addition, an event-triggered condition is defined in order to decide when the control signal is sent to the actuator located at the sensor node. This condition is assessed only when a sampled output is 
received from the sensor. Thus, unlike the condition included at the sensor, the condition defined at the base station is not periodically evaluated.

Next, the proposed scenario is described (more details can be found in section 7.3):

- When the periodic event-triggered condition is satisfied at the sensor, a new output $\left(w_{r}, w_{l}\right)_{k}^{N T}$ is sent to the base station. If no dropout occurs in the sensor-to-controller link, $d_{s c, k}^{N T}=1$, the output is received at the base station after a delay $\tau_{s c, k}^{N T}$. Then, the control algorithm computes the number of $N T$-periods elapsed between the previous and current outputs that are received by the base station. Let us name this amount of $N T$-periods as the multiplicity $m$ of the TVDRKF, which relates the sensing rate $1 / N T$ to the receiving rate at the base station $1 / \bar{N} T$, where $\bar{N}=m N$, being $N$ the multiplicity of the dual-rate sampled-data system. Since the data pattern received by the base station follows a non-uniform scheme, the value $m$, and in consequence $\bar{N}$, are time-varying.

- From $\bar{N}$, the gain $K(\bar{N})$ of the TVDRKF can be calculated in order to perform the correction of the estimator, resulting in the estimate of the state, $\hat{x}_{(k \mid k)}^{T}$, where, in general, the notation $\hat{x}_{(i \mid j)}^{T}$ will be used to define the estimate of the state $x_{i}^{T}$ based on the sensed process output up to time instant $j$, being $j \leq i$.

- From $\hat{x}_{(k \mid k)}^{T}$, the estimated, corrected output $\left(\hat{w}_{r}, \hat{w}_{l}\right)_{k}^{N T}$ and a set of $N$ estimated values inside the period $N T$ for the disturbance signal that affects each motor $\left\{\left(\hat{d}_{r}, \hat{d}_{l}\right)_{k}^{T},\left(\hat{d}_{r}, \hat{d}_{l}\right)_{k+1}^{T}, \cdots,\left(\hat{d}_{r}, \hat{d}_{l}\right)_{k+N-1}^{T}\right\}$ can be obtained. The set of estimated values of the disturbance will be later used by the dual-rate controller to finally tune the control action.

- From $\left(\hat{w}_{r}, \hat{w}_{l}\right)_{k}^{N T}$, and $\left(x_{r e f}, y_{r e f}, \theta_{r e f}\right)_{k}^{N T}$, which is the path reference, the Pure Pursuit path tracking algorithm calculates the rotational velocity command (dynamic reference) $\left(\hat{w}_{r, r e f}, \hat{w}_{l, r e f}\right)_{k}^{N T}$.

- From $\left(\hat{w}_{r}, \hat{w}_{l}\right)_{k}^{N T}$ and $\left(\hat{w}_{r, r e f}, \hat{w}_{l, r e f}\right)_{k}^{N T}$, the dual-rate controller is utilized to generate the set of $N$ current, estimated control actions $\left\{\left(\hat{u}_{r}, \hat{u}_{l}\right)_{k}^{T}\right.$, $\left.\left(\hat{u}_{r}, \hat{u}_{l}\right)_{k+1}^{T}, \cdots,\left(\hat{u}_{r}, \hat{u}_{l}\right)_{k+N-1}^{T}\right\}$ in order to achieve the desired control performance. The control signal is finally tuned by means of the set of $N$ estimated values of the disturbance $\left\{\left(\hat{d}_{r}, \hat{d}_{l}\right)_{k}^{T},\left(\hat{d}_{r}, \hat{d}_{l}\right)_{k+1}^{T}, \cdots\right.$, $\left.\left(\hat{d}_{r}, \hat{d}_{l}\right)_{k+N-1}^{T}\right\}$ in order to compensate for the actual ones $\left\{d_{k}^{T}, d_{k+1}^{T}, \cdots\right.$ ,$\left.d_{k+N-1}^{T}\right\}$ (where a unique signal is considered for both motors). 
- From $\left\{\left(\hat{u}_{r}, \hat{u}_{l}\right)_{k}^{T},\left(\hat{u}_{r}, \hat{u}_{l}\right)_{k+1}^{T}, \cdots,\left(\hat{u}_{r}, \hat{u}_{l}\right)_{k+N-1}^{T}\right\}$ and the model of the plant, the TVDRKF's prediction stage can be carried out, giving $\hat{x}_{(k+N \mid k)}^{T}$. Then, the next estimated output $\left(\hat{w}_{r}, \hat{w}_{l}\right)_{k+1}^{N T}$ and the next set of estimated disturbances $\left\{\left(\hat{d}_{r}, \hat{d}_{l}\right)_{k+N}^{T},\left(\hat{d}_{r}, \hat{d}_{l}\right)_{k+N+1}^{T}, \cdots,\left(\hat{d}_{r}, \hat{d}_{l}\right)_{k+2 N-1}^{T}\right\}$ can be calculated. From the estimated output, and taking into account the next path reference $\left(x_{r e f}, y_{r e f}, \theta_{r e f}\right)_{k+1}^{N T}$, the Pure Pursuit algorithm gets the next dynamic reference $\left(\hat{w}_{r, r e f}, \hat{w}_{l, r e f}\right)_{k+1}^{N T}$. Finally, the dual-rate controller is able to compute the next set of $N$ control actions $\left\{\left(\hat{u}_{r}, \hat{u}_{l}\right)_{k+N}^{T}\right.$ $\left.,\left(\hat{u}_{r}, \hat{u}_{l}\right)_{k+N+1}^{T}, \cdots,\left(\hat{u}_{r}, \hat{u}_{l}\right)_{k+2 N-1}^{T}\right\}$ from the estimated output, the dynamic reference, and the set of estimated disturbances. By means of this $h$-step ahead prediction algorithm, a set of $h N$ future, estimated values of the control signal $\left\{\left(\hat{u}_{r}, \hat{u}_{l}\right)_{k+N}^{T},\left(\hat{u}_{r}, \hat{u}_{l}\right)_{k+N+1}^{T}, \cdots,\left(\hat{u}_{r}, \hat{u}_{l}\right)_{k+h N-1}^{T}\right\}$ can be computed.

- When the event-triggered condition at the base station is satisfied, the current control signal $\left\{\left(\hat{u}_{r}, \hat{u}_{l}\right)_{k}^{T},\left(\hat{u}_{r}, \hat{u}_{l}\right)_{k+1}^{T}, \cdots,\left(\hat{u}_{r}, \hat{u}_{l}\right)_{k+N-1}^{T}\right\}$ and the $h N$ future ones $\left\{\left(\hat{u}_{r}, \hat{u}_{l}\right)_{k+N}^{T},\left(\hat{u}_{r}, \hat{u}_{l}\right)_{k+N+1}^{T}, \cdots,\left(\hat{u}_{r}, \hat{u}_{l}\right)_{k+h N-1}^{T}\right\}$ are sent to the actuator in a packet. If no dropout occurs in the controllerto-actuator link, $d_{c a, k}^{N T}=1$, the whole set of control actions is received at the sensor node after a delay $\tau_{c a, k}^{N T}$. Therefore, the actuator injects the current control action $\left\{\left(\hat{u}_{r}, \hat{u}_{l}\right)_{k}^{T},\left(\hat{u}_{r}, \hat{u}_{l}\right)_{k+1}^{T}, \cdots,\left(\hat{u}_{r}, \hat{u}_{l}\right)_{k+N-1}^{T}\right\}$, and the future ones $\left\{\left(\hat{u}_{r}, \hat{u}_{l}\right)_{k+N}^{T},\left(\hat{u}_{r}, \hat{u}_{l}\right)_{k+N+1}^{T}, \cdots,\left(\hat{u}_{r}, \hat{u}_{l}\right)_{k+h N-1}^{T}\right\}$ while no new packet is received for the $h N T$ future periods. In each $N T$ period, the actuation occurs at uniformly spaced instants $k N T+l T(l=$ $0,1, \cdots, N-1)$ under Zero Order Hold $(\mathrm{ZOH})$ conditions (i.e., $\left(\hat{u}_{r}, \hat{u}_{l}\right)_{k}^{T}$ is applied at $k N T,\left(\hat{u}_{r}, \hat{u}_{l}\right)_{k+1}^{T}$ is injected at $k N T+T$, and so on up to $\left(\hat{u}_{r}, \hat{u}_{l}\right)_{k+N-1}^{T}$, which is actuated at $\left.k N T+(N-1) T\right)$. That results in a uniform actuation pattern $\{0, T, \cdots,(N-1) T\}$ inside the sensor period $N T$. In conclusion, the working mode is to use estimated control actions when no new, actual ones are received by the sensor node, and replace the estimations with the actual actions when the packet is received. As external disturbance and measurement noise are faced by the TVDRKF, and an accurate system model is assumed, estimated and actual values are usually very similar. 


\subsection{Energy-efficient control solution design}

Next, each component of the control system is defined in detail.

\subsubsection{Plant modeling}

Considering state-space representation, the model of the plant at sampling period $T$ takes this form

$$
\begin{cases}x_{p, k+1}^{T} & =A_{p} x_{p, k}^{T}+B_{p} \hat{u}_{k}^{T}+B_{p} d_{k}^{T} \\ y_{k}^{T} & =C_{p} x_{p, k}^{T}+v_{k}^{T}\end{cases}
$$

where, for the sake of simplicity, let us name

- $y_{k}^{T}$ as the measurement, that is, the rotational velocity either for the right motor $w_{r, k}^{T}$ or for the left motor $w_{l, k}^{T}$, and

- $\hat{u}_{k}^{T}$ as the control signal, regardless of the motor $\left(\hat{u}_{r, k}^{T}\right.$ or $\left.\hat{u}_{l, k}^{T}\right)$.

In addition, $v_{k}^{T}$ is the measurement noise, $d_{k}^{T}$ is the disturbance signal, $x_{p, k}^{T}$ is the process state (regardless of the motor), and $A_{p}, B_{p}, C_{p}$ are matrices with suitable dimensions.

Using Z-transform at period $T$, the input-output plant model for the control signal (input) and the measurement (output) is represented as a discrete-time transfer function

$$
G_{p}(z)=Y^{T}(z) / U^{T}(z)
$$

being $z$ the $T$-unit operator.

Given a broadband noise $\tilde{w}_{k}^{T}$, the disturbance $d_{k}^{T}$ can be generated through the following dynamics

$$
\begin{cases}x_{d, k+1}^{T} & =A_{d} x_{d, k}^{T}+B_{d} \tilde{w}_{k}^{T} \\ d_{k}^{T} & =C_{d} x_{d, k}^{T}\end{cases}
$$


where $x_{d, k}^{T}$ is the disturbance state, and $A_{d}, B_{d}, C_{d}$ are matrices with proper dimensions. Considering (7.4) and (7.6), the system can be augmented as follows

$$
\begin{cases}{\left[\begin{array}{l}
x_{p, k+1}^{T} \\
x_{d, k+1}^{T}
\end{array}\right]} & =\left[\begin{array}{cc}
A_{p} & B_{p} C_{d} \\
0 & A_{d}
\end{array}\right]\left[\begin{array}{c}
x_{p, k}^{T} \\
x_{d, k}^{T}
\end{array}\right]+\left[\begin{array}{c}
B_{p} \\
0
\end{array}\right] \hat{u}_{k}^{T}+\left[\begin{array}{c}
0 \\
B_{d}
\end{array}\right] \tilde{w}_{k}^{T} \\
y_{k}^{T} & =\left[\begin{array}{ll}
C_{p} & 0
\end{array}\right]\left[\begin{array}{l}
x_{p, k}^{T} \\
x_{d, k}^{T}
\end{array}\right]+v_{k}^{T} \\
d_{k}^{T} & =\left[\begin{array}{ll}
0 & C_{d}
\end{array}\right]\left[\begin{array}{l}
x_{p, k}^{T} \\
x_{d, k}^{T}
\end{array}\right]\end{cases}
$$

Assuming the following notation

$$
\begin{aligned}
A & =\left[\begin{array}{cc}
A_{p} & B_{p} C_{d} \\
0 & A_{d}
\end{array}\right], B=\left[\begin{array}{c}
B_{p} \\
0
\end{array}\right], B_{\tilde{w}}=\left[\begin{array}{c}
0 \\
B_{d}
\end{array}\right] \\
C & =\left[\begin{array}{ll}
C_{p} & 0
\end{array}\right], \bar{C}=\left[\begin{array}{ll}
0 & C_{d}
\end{array}\right], x_{k}^{T}=\left[\begin{array}{l}
x_{p, k}^{T} \\
x_{d, k}^{T}
\end{array}\right]
\end{aligned}
$$

the augmented system in (7.7) can be expressed as

$$
\begin{cases}x_{k+1}^{T} & =A x_{k}^{T}+B \hat{u}_{k}^{T}+B_{\tilde{w}} \tilde{w}_{k}^{T} \\ y_{k}^{T} & =C x_{k}^{T}+v_{k}^{T} \\ d_{k}^{T} & =\bar{C} x_{k}^{T}\end{cases}
$$

that admits a lifted representation [41] such as

$$
\begin{cases}x_{k+N}^{T} & =A^{N} x_{k}^{T}+\sum_{c=0}^{N-1} A^{N-1-c} B \hat{u}_{k+c}^{T}+\sum_{c=0}^{N-1} A^{N-1-c} B_{\tilde{w}} \tilde{w}_{k+c}^{T} \\ y_{k}^{T} & =C x_{k}^{T}+v_{k}^{T}\end{cases}
$$

which can be equivalently seen as a dual-rate sampled-data system, where the output at period $N T, y_{k}^{N T}$, is obtained from a sequence of inputs at period $T$, $\left\{\hat{u}_{k}^{T}, \hat{u}_{k+1}^{T}, \cdots, \hat{u}_{k+N-1}^{T}\right\}$. 


\subsubsection{Event-triggered conditions}

Let us denote $\beta_{k}^{N T} \in\{0,1\}$ as the scheduling variable at the sensor. When $\beta_{k}^{N T}=1$, the sensor data $y_{k}^{N T}$ is transmitted, and when $\beta_{k}^{N T}=0$, no transmission is carried out. The variable $\bar{y}_{k}^{N T}$ is used to store the latest sensor data in such a way that:

$$
\bar{y}_{k}^{N T}=\beta_{k}^{N T} y_{k}^{N T}+\left(1-\beta_{k}^{N T}\right) \bar{y}_{k-1}^{N T}, \text { for } k \in \mathbb{N}_{\geq 1}
$$

and where $\bar{y}_{0}^{N T}=y_{0}^{N T}$. By means of a discrete-time version of the so-called mixed triggered mechanism [42] based on the process output $y_{k}^{N T}$, the periodic event-triggered condition at the sensor can be implemented. In this way, the measurement $y_{k}^{N T}$ is sent to the base station through the network (i.e., $\beta_{k}^{N T}=$ 1) when

$$
\left\|\bar{y}_{k-1}^{N T}-y_{k}^{N T}\right\|^{2}>\sigma_{s}\left\|y_{k}^{N T}\right\|^{2}+\delta_{s}, \text { for } k \in \mathbb{N}_{\geq 1}
$$

where $\bar{y}_{0}^{N T}=y_{0}^{N T}$, and $\sigma_{s}$ and $\delta_{s}$ are positive constants. Note that usually, $\sigma_{s}$ is chosen to take values close to zero or even zero; in any case, smaller than one [43].

When $\beta_{k}=1$, the control algorithm calculates the control signal $\left\{\hat{u}_{k}^{T}, \hat{u}_{k+1}^{T}\right.$, $\left.\cdots, \hat{u}_{k+h N-1}^{T}\right\}$. Let us denote $\gamma_{k} \in\{0,1\}$ as the scheduling variable for the control signal. When $\gamma_{k}=1$, the signal is transmitted, and when $\gamma_{k}=0$, no transmission is carried out. The variable $\overline{\hat{u}}_{k}^{T}$ is used to store the first value $\hat{u}_{k}^{T}$ of the last sent control signal in such a way that:

$$
\overline{\hat{u}}_{k}^{T}=\gamma_{k} \hat{u}_{k}^{T}+\left(1-\gamma_{k}\right) \overline{\hat{u}}_{k-1}^{T}, \text { for } k \in \mathbb{N}_{\geq 1}
$$

where $\overline{\hat{u}}_{0}^{T}=\hat{u}_{0}^{T}$. By means of the following mixed triggered mechanism, the control signal is transmitted to the sensor node:

$$
\left\|\overline{\hat{u}}_{k-1}^{T}-\hat{u}_{k}^{T}\right\|^{2}>\sigma_{c}\left\|\hat{u}_{k}^{T}\right\|^{2}+\delta_{c}, \text { for } k \in \mathbb{N}_{\geq 1}
$$

where $\overline{\hat{u}}_{0}^{T}=\hat{u}_{0}^{T}$, and $\sigma_{c}$ and $\delta_{c}$ are positive constants. As previously commented with regard to the choice of $\sigma_{s}$, note that typically $\sigma_{c}$ is chosen to be less than one. 
Observe that the feedback loop is only closed from sensor node to base station, and back to sensor node, when the conditions (7.11) and (7.13) hold (and hence, $\beta_{k}=\gamma_{k}=1$ ). If one of them is not satisfied (i.e. $\beta_{k}=0$ or $\gamma_{k}=0$ ), then the control signal is not updated. Nevertheless, the future control actions $\left\{\hat{u}_{k+N}^{T}, \ldots, \hat{u}_{k+h N-1}^{T}\right\}$, which were previously sent, can be used by the actuator in order to keep satisfactory control properties.

\subsubsection{Time-varying dual-rate Kalman filter}

Considering $\tilde{w}_{k}^{T}$ and $v_{k}^{T}$ as zero-mean white noises for the single-rate system in (7.8), the best linear estimation for $x_{k}^{T}$ in the sense of mean square error is provided by conventional Kalman filter [44]. When different rates are involved in the control system, a multi-rate Kalman filter containing corrections and predictions [45] is required. In our work, dual-rate control and Periodic Event-Triggered Control (PETC) are integrated in order to save system resources (energy and bandwidth). As a consequence of using PETC, not all the measurements are sent to the base station, which results in a Time-Varying Dual-Rate Kalman Filter (TVDRKF) [24-26]. From Extended State Observer (ESO) [46] and multi-rate Kalman filter [45, 47] techniques, the proposed TVDRKF is able to estimate the plant measurement and disturbance, following a non-uniform dual-rate scheme.

Taking into account (7.11), the outputs sampled at the slow rate $1 / N T$ arrive at the base station at a slower rate $1 / \bar{N} T$ in a non-uniform fashion. The multiplicity $m=\bar{N} / N$ of the TVDRKF was previously introduced in section 7.2. It can be now enunciated from the previous output received by the base station $\bar{y}_{k-1}^{N T}$ (which was sampled in time, say, $k_{l s} N T$ ) and the current received output $\bar{y}_{k}^{N T}=y_{k}^{N T}$. Hence

$$
m=k-k_{l s}
$$

Due to the non-uniform nature of the pattern, $m$ and in consequence $\bar{N}$ will be time-varying. From the current received output, the TVDRKF can perform the correction (and filtering) stage, and then, the $h N$-step ahead state predictions at the fast rate $1 / T$. In order to do it, the lifted representation in (7.9) can be taken, replacing $N$ with $\bar{N}=m N$.

From the notation $\hat{x}_{(i \mid j)}^{T}$ introduced in section 7.2 , which is used to denote the estimate of the state $x_{i}^{T}$ based on the output previously sensed at instant $j$, the 
correction and prediction stages of the TVDRKF can be designed as follows (illustrated in Figure 7.2):

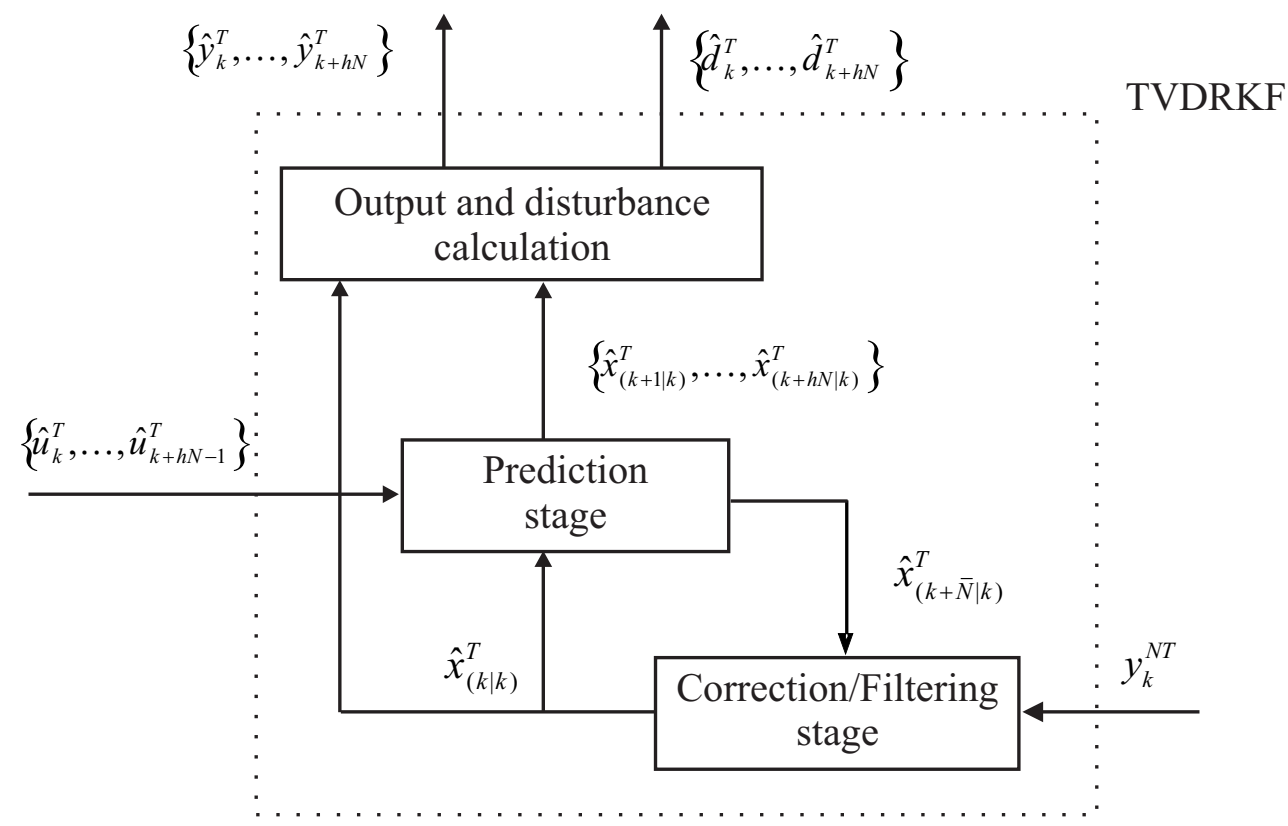

Figure 7.2: Structure of the Time-Varying Dual-Rate Kalman Filter

- Correction stage: when a new measurement $y_{k}^{N T}$ is available at time $k N T$, it can be used to make correction for the prediction $\hat{x}_{(k \mid k)}^{N T}$. Expressing this stage at period $T$ results in

$$
\begin{aligned}
\hat{x}_{(k \mid k)}^{T} & =\hat{x}_{(k \mid k-\bar{N})}^{T} \\
& +K(\bar{N})\left[y_{k}^{T}-C \hat{x}_{(k \mid k-\bar{N})}^{T}\right]
\end{aligned}
$$

where, from multi-rate Kalman filter design techniques, the time-varying $K(\bar{N})$ can be calculated

$$
\begin{aligned}
K(\bar{N}) & =M_{k+1} C^{\mathrm{t}}\left[C M_{k+1} C^{\mathrm{t}}+V\right]^{-1} \\
M_{k+1} & =A^{\bar{N}} M_{k}\left(A^{\bar{N}}\right)^{\mathrm{t}}+W_{e} \\
& -A^{\bar{N}} M_{k} C^{\mathrm{t}}\left[C M_{k} C^{\mathrm{t}}+V\right]^{-1} C M_{k}\left(A^{\bar{N}}\right)^{\mathrm{t}}
\end{aligned}
$$


where $(\cdot)^{\mathrm{t}}$ denotes transpose function, $V=\operatorname{Cov}(v)$ and

$$
\begin{aligned}
W_{e} & =\operatorname{Cov}\left(\sum_{c=0}^{\bar{N}-1} A^{\bar{N}-1-c} B_{\tilde{w}} \tilde{w}_{k+c}\right)=\operatorname{Cov}\left(w_{e}\right) \\
& =E\left\{w_{e} w_{e}^{\mathrm{t}}\right\} \\
& =\left(\sum_{c=0}^{\bar{N}-1} A^{\bar{N}-1-c} B_{\tilde{w}}\right) W\left(\sum_{c=0}^{\bar{N}-1} A^{\bar{N}-1-c} B_{\tilde{w}}\right)^{\mathrm{t}}
\end{aligned}
$$

where $E\{\cdot\}$ denotes the expectation, and $W=\operatorname{Cov}(\tilde{w})$.

- Prediction stage: when no new measurement is available between the previous sensed output $y_{k}^{N T}$ and the next sensed output $y_{k+m}^{N T}$ (or, equivalently at period $T$, between the previous sensed output $y_{k}^{T}$ and the next sensed output $\left.y_{k+\bar{N}}^{T}\right)$, the best estimates $\hat{x}_{(k+l \mid k)}^{T}(l=1, \ldots, h N)$ are obtained from the open-loop dynamics based prediction:

$$
\hat{x}_{(k+l \mid k)}^{T}=A^{l} \hat{x}_{(k \mid k)}^{T}+\sum_{c=0}^{l-1} A^{l-1-c} B \hat{u}_{k+c}^{T}
$$

where $h$ must be defined to fulfill $h N \geq \bar{N}_{\text {max }}$, being $\bar{N}_{\text {max }}$ the maximum value of $\bar{N}$. This definition ensures the computation of the TVDRKF's correction stage, since the required ahead state $\hat{x}_{(k+\bar{N} \mid k)}^{T}$ will be available. Moreover, from (7.18), the set of $h N$ estimated outputs and disturbances (regardless of the motor) can be calculated:

$$
\begin{aligned}
& \hat{y}_{k+l}^{T}=C \hat{x}_{(k+l \mid k)}^{T} \\
& \hat{d}_{k+l}^{T}=\bar{C} \hat{x}_{(k+l \mid k)}^{T}
\end{aligned}
$$

\subsubsection{Pure Pursuit path tracking algorithm}

From the desired kinematic reference $\left(x_{r e f}, y_{r e f}, \theta_{r e f}\right)_{k}^{N T}$ and the plant output generated by the TVDRKF at period $N T, \hat{y}_{k}^{N T}$, which is composed of corrected estimations obtained when measurements are received by the base station (i.e., when the condition (7.11) holds, and hence, $\beta_{k}=1$ ), the Unmanned Ground Vehicle (UGV) is able to infer its current position using a 
path tracking algorithm. In this work, Pure Pursuit via an odometry technique is chosen. Basically, the Pure Pursuit algorithm tries to determine the velocity and turning conditions every time interval so that the UGV follows a prescribed trajectory [48]. Odometry is the simplest way for dead reckoning implementation, which is a method used to compute the vehicle's current position from a previous position, knowing additionally in advance robot speed and path in a certain period of time. Odometry is usually based on motors rotation considering their encoders measurements.

The path tracking algorithm generates the dynamic reference based on the rotational velocity for both wheels, i.e., $\left(\hat{w}_{r, r e f}, \hat{w}_{l, r e f}\right)_{k}^{N T} \equiv \hat{y}_{r e f, k}^{N T}$, in order to properly reach the next point of the desired trajectory. The UGV path tracking is composed of a set of $h$ future dynamic references, $\left\{\hat{y}_{r e f, k}^{N T}, \hat{y}_{r e f, k+1}^{N T}\right.$ $\left., \cdots, \hat{y}_{r e f, k+h}^{N T}\right\}$. In order to establish these references, the trajectory that must be followed by the UGV is required to be known in advance. This task is developed by the reference generator, which is in charge of providing the Pure Pursuit algorithm with the sequence of $h$-step ahead kinematic references, $\left\{\left(x_{r e f}, y_{r e f}, \theta_{r e f}\right)_{k}^{N T},\left(x_{r e f}, y_{r e f}, \theta_{r e f}\right)_{k+1}^{N T}, \cdots,\left(x_{r e f}, y_{r e f}, \theta_{r e f}\right)_{k+h}^{N T}\right\}$.

\subsubsection{Differential kinematics}

While direct kinematics specifies the positions that the UGV is able to reach by giving the wheel speed, differential kinematics establishes relations between motion (velocity) in joint space and motion (linear/angular velocity) in task space (e.g., Cartesian space). A two-wheel UGV rotates with respect to a point located in some place of the axis, which is shared by the two wheels. This point is known as Instantaneous Rotation Center (IRC), that is the point with zero velocity at a particular instant of time in a body undergoing planar movement. IRC varies according to wheel speed variations.

Each wheel follows a trajectory with the same rotational velocity $\omega_{R}$ with respect to the IRC. This fact implies that the linear velocity of the wheels are

$$
\begin{aligned}
& v_{r}=\omega_{R}(R+l / 2) \\
& v_{l}=\omega_{R}(R-l / 2)
\end{aligned}
$$

where $v_{r}$ and $v_{l}$ are respectively the linear velocity for the right and left motors, $l$ is the distance between the two wheels, and $R$ is the distance between the IRC and the middle point of the distance between the wheels. Therefore 


$$
R=\frac{l}{2} \cdot \frac{v_{l}+v_{r}}{v_{r}-v_{l}}
$$

and the rotational velocity of the robot $\omega_{R}$ is

$$
\omega_{R}=\frac{v_{r}-v_{l}}{l}
$$

\subsubsection{Path tracking algorithm}

The Pure Pursuit algorithm is based on the computation of the curvature $\bar{\gamma}$ that a vehicle must adopted from its current position $(x, y)$ to a target position $(x+\Delta x, y+\Delta y)$. As depicted in Figure 7.3, for this purpose, a circle of radius $r$ that joints both points is defined. The center of the circle is placed in $x+\Delta x+d$. In addition, the distance to the target point is $L$. Therefore, it is possible to express

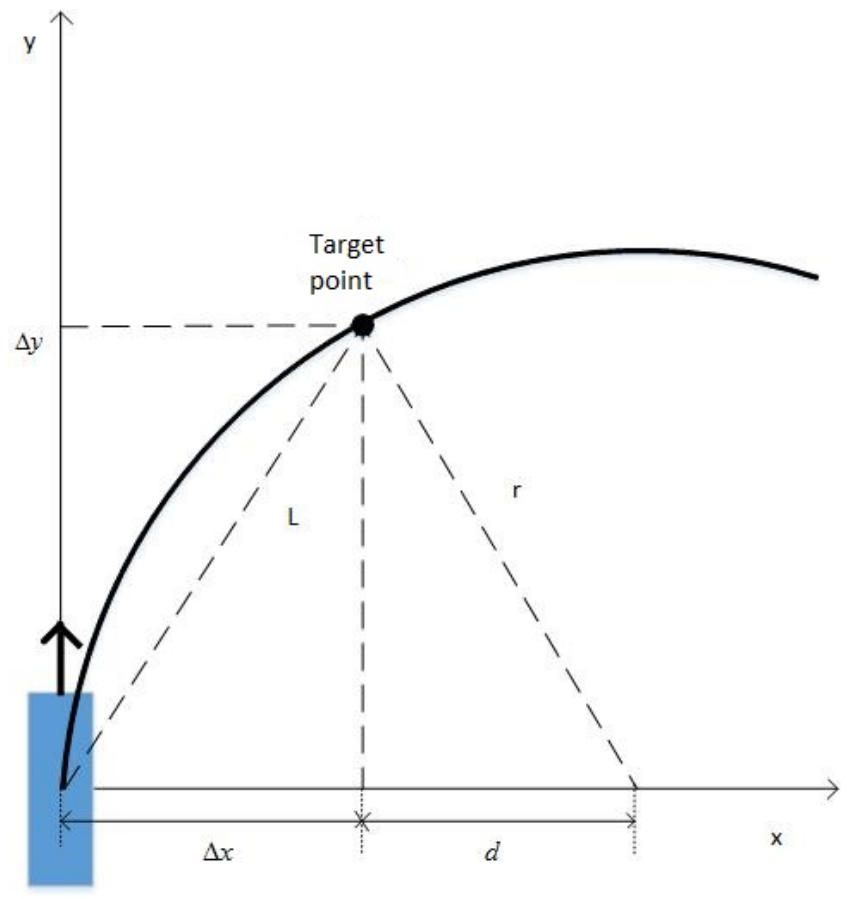

Figure 7.3: Circumference between current point and target point 


$$
\begin{aligned}
r & =\Delta x+d \\
L^{2} & =(\Delta x)^{2}+(\Delta y)^{2}
\end{aligned}
$$

being the curvature

$$
\bar{\gamma}=\frac{(\Delta x)^{2}+(\Delta y)^{2}}{2 \Delta x}
$$

and the so-called Pure Pursuit control law

$$
\bar{k}=\frac{1}{\bar{\gamma}}=\frac{2 \Delta x}{L^{2}}
$$

As it can be seen, the control law $\bar{k}$ is proportional to the lateral shift, and inversely proportional to the square of $L$. Assuming robot coordinates for the current position $\left(x_{R}, y_{R}\right)$ and for the target, reference position $\left(x_{R, r e f}, y_{R, r e f}\right)$, and the angle $\theta_{R}$ as the current vehicle heading, the Pure Pursuit control law $\bar{k}$ can be expressed as follows

$$
\bar{k}=2 \frac{\left(y_{R, r e f}-y_{R}\right) \cos \left(\theta_{R}\right)-\left(x_{R, r e f}-x_{R}\right) \sin \left(\theta_{R}\right)}{\left(x_{R, r e f}-x_{R}\right)^{2}+\left(y_{R, r e f}-y_{R}\right)^{2}}
$$

For a desired linear velocity $v_{r e f}$, the rotational velocity reference $w_{r e f}$ can be calculated as

$$
\omega_{\text {ref }}=v_{\text {ref }} \bar{k}
$$

The path tracking algorithm requires to determine one point located at a minimum distance from the current point, i.e., the so-called Look Ahead Distance (LAD), not considering the nearest points in the prescribed trajectory. This procedure avoids a severe correction, and hence, it leads to a soft movement.

Finally, these are the steps to be followed when the main loop of the Pure Pursuit algorithm is implemented (depicted in more detail in Figure 7.4):

1. UGV rotational and linear velocity calculation from system output estimations and corrections provided by the TVDRKF at period NT (i.e., calculation of $\omega_{R, k}^{N T}$ and $v_{R, k}^{N T}$, respectively, from $\hat{w}_{r, k}^{N T}$ and $\hat{w}_{l, k}^{N T}$, that is, 


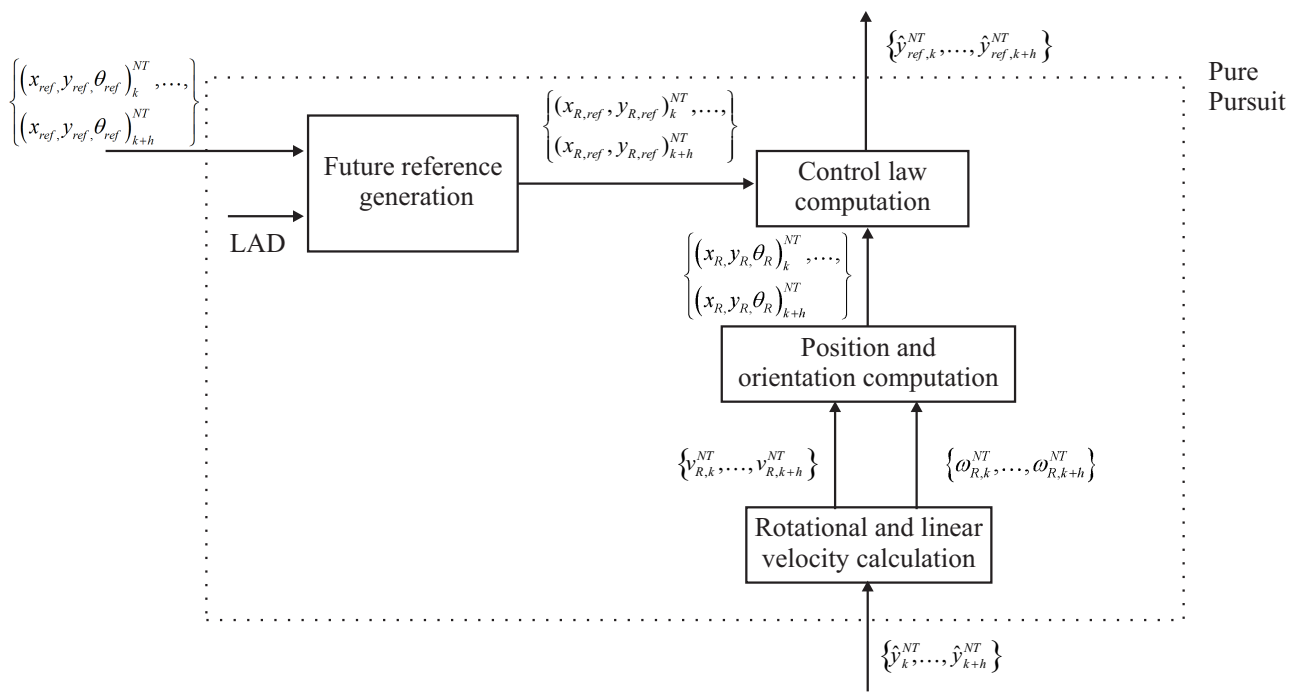

Figure 7.4: Structure of the Pure Pursuit path tracking algorithm

$\hat{y}_{k}^{N T}$ ). This is obtained from (7.20), where $R+l / 2 \approx r$, being $r$ the wheel radius, and (7.22)

$$
\begin{aligned}
v_{r, k}^{N T} & =\hat{w}_{r, k}^{N T} r \\
v_{l, k}^{N T} & =\hat{w}_{l, k}^{N T} r \\
v_{R, k}^{N T} & =\frac{v_{r, k}^{N T}+v_{l, k}^{N T}}{2} \\
\omega_{R, k}^{N T} & =\frac{v_{r, k}^{N T}-v_{l, k}^{N T}}{l}
\end{aligned}
$$

2. UGV position and orientation computation in the time period NT

$$
\begin{aligned}
x_{R, k}^{N T} & =x_{R, k-1}^{N T}+v_{R, k}^{N T} N T \cos \left(\theta_{R, k-1}^{N T}+\omega_{R, k}^{N T} N T\right) \\
y_{R, k}^{N T} & =y_{R, k-1}^{N T}+v_{R, k}^{N T} N T \sin \left(\theta_{R, k-1}^{N T}+\omega_{R, k}^{N T} N T\right) \\
\theta_{R, k}^{N T} & =\theta_{R, k-1}^{N T}+\omega_{R, k}^{N T} N T
\end{aligned}
$$

for $k \in \mathbb{N}_{\geq 1}$, where $\left(x_{R, 0}^{N T}, y_{R, 0}^{N T}, \theta_{R, 0}^{N T}\right)$ is the initial position and orientation. 
3. Generation of the future reference for the robot, $\left(x_{R, r e f}, y_{R, r e f}\right)_{k}^{N T}$ : From the desired kinematic reference and the Look Ahead Distance (LAD), the nearest point to the future path tracking that is located far away from the LAD is calculated.

4. Control law computation: from $\left(x_{R, r e f}, y_{R, r e f}\right)_{k}^{N T}$ and $\left(x_{R}, y_{R}, \theta_{R}\right)_{k}^{N T}$, the control law $\bar{k}$ is computed at period $N T$ by using $((7.26))$, and then, $\omega_{r e f}^{N T}$ is calculated for each wheel by using $((7.27))$ and from a desired $v_{r e f}^{N T}$. Finally, from these data, $\left(\hat{w}_{r, r e f}, \hat{w}_{l, r e f}\right)_{k}^{N T} \equiv \hat{y}_{r e f, k}^{N T}$ can be calculated

$$
\begin{aligned}
\left(\hat{w}_{r, r e f}\right)_{k}^{N T} & =\frac{v_{r e f}^{N T}+\omega_{r e f}^{N T} b}{r} \\
\left(\hat{w}_{l, r e f}\right)_{k}^{N T} & =\frac{v_{r e f}^{N T}-\omega_{r e f}^{N T} b}{r}
\end{aligned}
$$

\subsubsection{Dual-rate controller}

In this work, in order to reach the desired control performance, a dual-rate controller is used. From $N T$-period signals such as the dynamic reference $\hat{y}_{r e f, k}^{N T}$ generated by the Pure Pursuit path tracking algorithm, and the estimated, corrected output $\hat{y}_{k}^{N T}$ obtained by the TVDRKF, the dynamic dual-rate controller computes $N$ control actions at period $T$ for each wheel, $U_{k}^{T}=\left\{\hat{u}_{k}^{T}\right.$, $\left.\hat{u}_{k+1}^{T}, \cdots, \hat{u}_{k+N-1}^{T}\right\}$. This control signal is finally tuned by adding the disturbance estimations generated by the TVDRKF at period $T,\left\{\hat{d}_{k}^{T}, \hat{d}_{k+1}^{T}, \cdots\right.$ , $\left.\hat{d}_{k+N-1}^{T}\right\}$. Following this operation mode for the next $h$ dynamic references and outputs, $\left\{\hat{y}_{r e f, k+1}^{N T}, \cdots, \hat{y}_{r e f, k+h}^{N T}\right\}$ and $\left\{\hat{y}_{k+1}^{N T}, \cdots, \hat{y}_{k+h}^{N T}\right\}$, respectively, and the next $h N$ disturbances $\left\{\hat{d}_{k+N}^{T}, \cdots, \hat{d}_{k+h N-1}^{T}\right\}$, the set of future control actions $\left\{\hat{u}_{k+N}^{T}, \cdots, \hat{u}_{k+h N-1}^{T}\right\}$ can be obtained.

Different alternatives can be followed to design a dual-rate controller (see, e.g., in $[29,30])$. In this case, the model-based dual-rate controller design described in [29] is chosen, where the structure of the controller includes (see in Figure 7.5)

- a slow-rate subcontroller $G_{1}^{N T}\left(z^{N}\right)=u_{1, k}^{N T} / e_{k}^{N T}$,

- a digital hold $H^{N T, T}(z)=u_{1, k}^{T} /\left[u_{1, k}^{N T}\right]^{T}$, and

- a fast-rate subcontroller $G_{2}^{T}(z)=U_{k}^{T} / u_{1, k}^{T}$. 


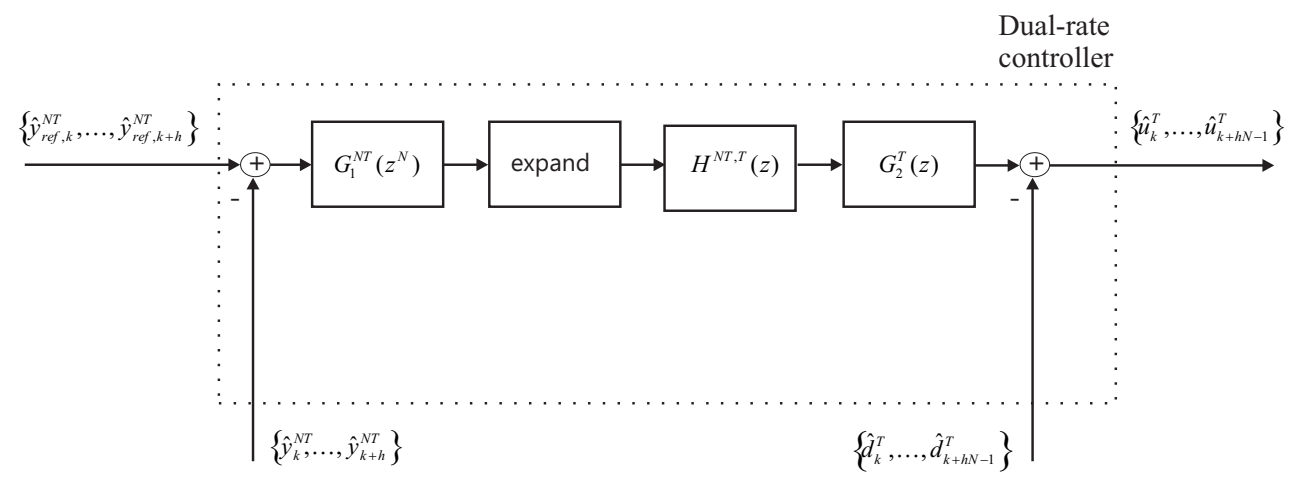

Figure 7.5: Structure of the dual-rate controller

where the input of $G_{1}^{N T}\left(z^{N}\right)$ is the error signal $e_{k}^{N T}=\hat{y}_{r e f, k}^{N T}-\hat{y}_{k}^{N T}$, and note that the output of $G_{1}^{N T}\left(z^{N}\right)$ (i.e, $u_{1, k}^{N T}$ ) is expanded $\left[u_{1, k}^{N T}\right]^{T}$ before being injected to the digital hold $H^{N T, T}(z)$. The expand operation implies to fill the slow-rate signal with zeros at the fast-rate instants (more details can be found in [29]). Then, the digital hold obtains its output $u_{1, k}^{T}$ by means of

$$
H^{N T, T}(z)=\frac{1-z^{-N}}{1-z^{-1}}
$$

which in conclusion results in the subcontroller output $u_{1, k}^{N T}$ repeated $N$ times. From the consideration of $M(s)$ as the desired closed-loop control performance of the original continuous-time system design, the subcontrollers $G_{1}^{N T}\left(z^{N}\right)$ and $G_{2}^{T}(z)$ will be designed as follows

$$
\begin{aligned}
G_{1}^{N T}\left(z^{N}\right) & =\frac{1}{1-M\left(z^{N}\right)} \\
G_{2}^{T}(z) & =\frac{M(z)}{G_{p}(z)}
\end{aligned}
$$

where $G_{p}(z)$ was presented in (7.5) and comes from the discretization of the continuous-time plant model at period $T$ under $\mathrm{ZOH}$ conditions, and $M(z)$ and $M\left(z^{N}\right)$ are the discretization of $M(s)$ at periods $T$ and $N T$, respectively, using $\mathrm{ZOH}$ techniques as well. This procedure leads to a behavior that perfectly matches $M(s)$ in the sample points at period $N T$, but sometimes (if $N T$ is too large) it can introduce a ripple between samples. The way to overcome 
this ripple is also described in [29], and it is based on modifying the fast-rate subcontroller design in this way

$$
G_{2}^{T}(z)=\frac{G_{R}(z)}{1+G_{R}(z) G_{p}(z)}
$$

being $G_{R}(z)$ the discrete-time version at period $T$ of the original continuoustime controller design. By using (7.39), the dual-rate controller does not cancel the numerator of the process transfer function $G_{p}(z)$, avoiding the ripple.

\subsection{Cost indexes for control performance and resource usage}

In this section, certain cost indexes closely related to control performance and resource usage will be presented. By means of these indexes, the energyefficient control proposal may be compared with the conventional time-triggered control strategy. Regarding control performance, similarly to [49], these three cost indexes will be used:

- $J_{1}$, which is based on the $\ell_{2}$-norm, and its goal is to provide a measure about how accurately the path is followed

$$
J_{1}=\sum_{k=1}^{l} \min _{1 \leq k^{\prime} \leq l} \sqrt{\left(x_{R, k}^{N T}-x_{r e f, k^{\prime}}^{N T}\right)^{2}+\left(y_{R, k}^{N T}-y_{r e f, k^{\prime}}^{N T}\right)^{2}}
$$

where $l$ is the number of iterations required by the UGV to reach the final point of the path, $\left(x_{R}, y_{R}\right)_{k}^{N T}$ is the current UGV position, which was defined in (7.32)-(7.34), and $\left(x_{r e f}, y_{r e f}\right)_{k^{\prime}}^{N T}$ is the nearest kinematic position reference to the current UGV position.

- $J_{2}$, which is based on the $\ell_{\infty}$-norm, and is defined to know the maximum difference between the desired path and the current UGV position

$$
J_{2}=\max _{1 \leq k \leq l}\left\{\min _{1 \leq k^{\prime} \leq l} \sqrt{\left(x_{R, k}^{N T}-x_{r e f, k^{\prime}}^{N T}\right)^{2}+\left(y_{R, k}^{N T}-y_{r e f, k^{\prime}}^{N T}\right)^{2}}\right\}
$$

- $J_{3}$, which measures the total amount of time (in seconds) elapsed to arrive at the final destination: 


$$
J_{3}=l N T
$$

To analyze the reduction of the resource usage in the energy-efficient control solution compared to the traditional time-triggered control, the cost index $J_{4}$ is defined by making use of the number of transmitted packets (NoT) in each case: $N o T_{\mathrm{EEC}}$ for the energy-efficient control, and $N o T_{\mathrm{TTC}}$ for the timetriggered control. In this way, $J_{4}$ (in \%) can be expressed as

$$
J_{4}=\frac{N o T_{\mathrm{EEC}}}{N o T_{\mathrm{TTC}}} \cdot 100 \% .
$$

\subsection{Simulation results via Truetime}

In this section, the main advantages of the energy-efficient control solution compared to the time-triggered one will be shown. The study will be focused on the tradeoff between resource usage and control properties. The section is split into two parts. Firstly, important data used for the simulation will be presented (transfer function, delay distribution, control parameters, and so on). Secondly, the cost indexes introduced in section 7.4 will be evaluated by means of a Truetime application [37].

\subsubsection{Application data}

Considering a similar model for both wheel motors, the model is described by means of this transfer function

$$
G_{p}(s)=\frac{0.1276}{0.1235 s+1}
$$

where the output is in $\mathrm{rad} / \mathrm{s}$, and the input in $\mathrm{V}$.

From previous off-line experiences on this WSN framework [49], different roundtrip time delay distributions, which can be modeled such as in (7.2), lead to consider a maximum time delay $\tau_{\max }$ slightly less than $200 \mathrm{~ms}$. Then, as commented in subsection 7.2.1, the sensor period is chosen as $N T=0.2 \mathrm{~s}$ in order to ensure no packet disorder. As it will be later detailed, in this case, choosing $N=2$ allows the UGV to accurately track the path. 
In this simulation, let us assume the packet dropout probability as $p_{s c}=0.1$ and $p_{c a}=0.3$ in (7.3).

The discrete-time controller design comes from the discretization at different periods of this continuous-time PID controller, which is designed following classical procedures $[50,51]$ and this typical configuration

$$
u(t)=K_{p}\left[e(t)+\frac{1}{T_{i}} \int_{0}^{t} e(\tau) d \tau\right]
$$

where $K_{p}=6$ and $T_{i}=0.12$ in order to achieve some specifications. The single-rate controllers are

$$
\begin{aligned}
G_{r}^{N T}\left(z^{N}\right) & =\frac{6 z+4}{z-1} \\
G_{r}^{T}(z) & =\frac{6 z-1}{z-1}
\end{aligned}
$$

The dual-rate controller is obtained by means of $((7.37))$ and $((7.39))$, bringing about

$$
\begin{aligned}
G_{1}^{N T}\left(z^{N}\right) & =\frac{z^{2}-0.4734 z+0.05731}{z^{2}-1.191 z+0.1914} \\
G_{2}^{T}(z) & =\frac{6.576 z^{2}-5.78 z+1.27}{z^{2}-0.9578 z+0.2394}
\end{aligned}
$$

The disturbance, which is defined at period $T$ as in (7.6), is

$$
\begin{aligned}
A_{d} & =\left[\begin{array}{cc}
0.9993 & 0.09994 \\
-0.0142 & 0.9985
\end{array}\right] \\
B_{d} & =\left[\begin{array}{c}
3.769 \cdot 10^{-5} \\
0.7535 \cdot 10^{-3}
\end{array}\right] \\
C_{d} & =\left[\begin{array}{ll}
0 & 1 \cdot 10^{5}
\end{array}\right]
\end{aligned}
$$

The Time-Varying Dual-Rate Kalman Filter (TVDRKF) is designed considering the augmented state resulting from the consequent state space realization (7.4) for (7.44), and from the disturbance (7.49). 
The positive constants in (7.11) and (7.13) for the event-triggered conditions will be respectively $\delta_{s}=0.01, \sigma_{s}=0$, and $\delta_{c}=0.5, \sigma_{c}=0$.

Finally, the reference to be followed includes a sequence of four right angles.

\subsubsection{Truetime simulation and cost function evaluation}

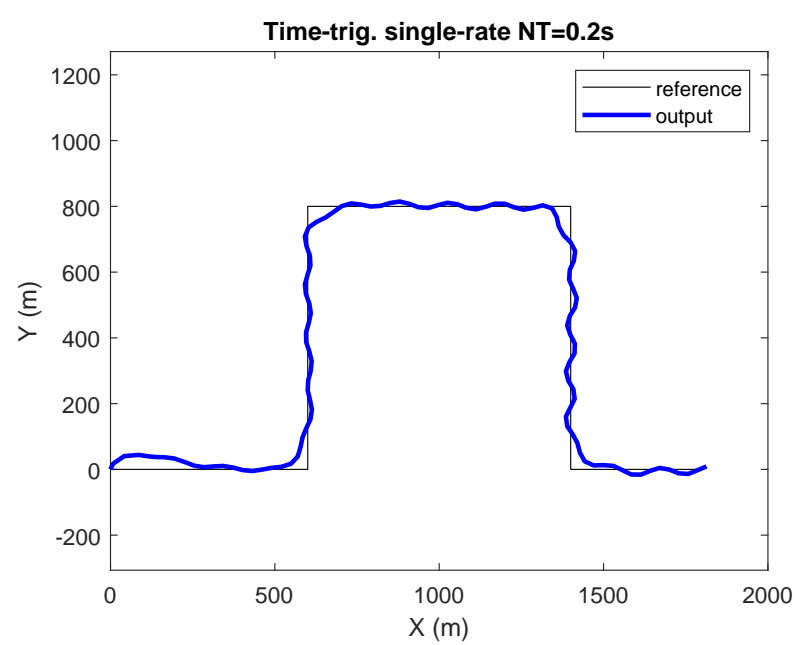

Figure 7.6: Time-triggered single-rate control at period $N T$ (no noise, no disturbance, no delays, no dropouts)

Starting from a time-triggered single-rate control scenario with neither noise nor disturbance, and neither time-varying delays nor packet dropouts, and using the controller at period $N T=0.2 \mathrm{~s}$ in $(7.45)$, some performance worsening is observed when the UGV tries to track the path (depicted in Figure 7.6) compared to the single-rate version at period $T=0.1 \mathrm{~s}$ in (7.46) (shown in Figure 7.7). Let us consider the performance reached at period $T$ as the nominal, desired one. Taking into account the dual-rate controller in (7.47)-(7.48), the time-triggered dual-rate control system is able to maintain a satisfactory control performance, very similar to the nominal one (as shown in Figure 7.8). But, including time-varying delays and packet dropouts, the performance is clearly worsened, becoming unstable (see in Figure 7.9).

If the TVDRKF is incorporated into the time-triggered dual-rate control system, where packet-based control is also integrated, the performance is clearly improved (illustrated in Figure 7.10), being very similar to the nominal one, despite additionally including both measurement noise and external distur- 


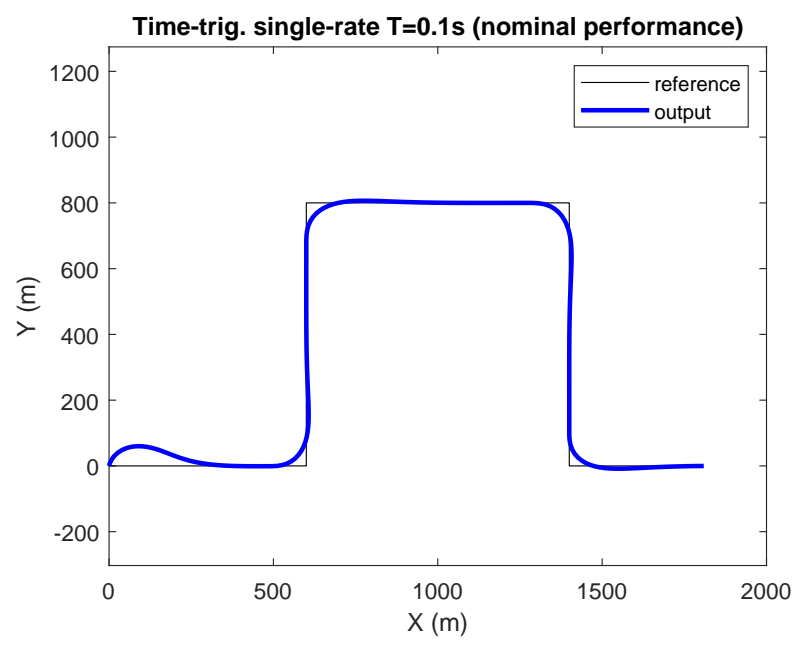

Figure 7.7: Time-triggered single-rate control at period $T$ (no noise, no disturbance, no delays, no dropouts). Nominal performance

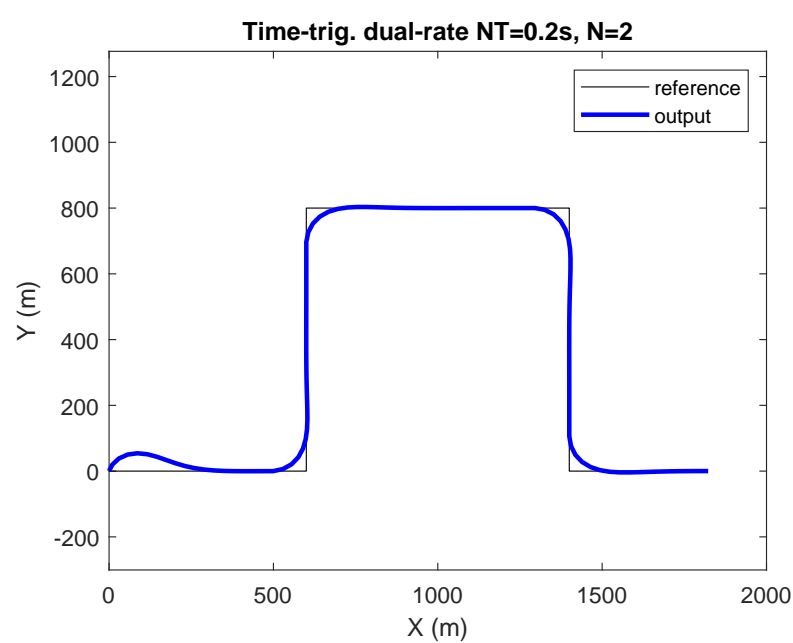

Figure 7.8: Time-triggered dual-rate control (no noise, no disturbance, no delays, no dropouts)

bance. Figure 7.11 shows that the disturbance estimation accurately follows the actual disturbance. 


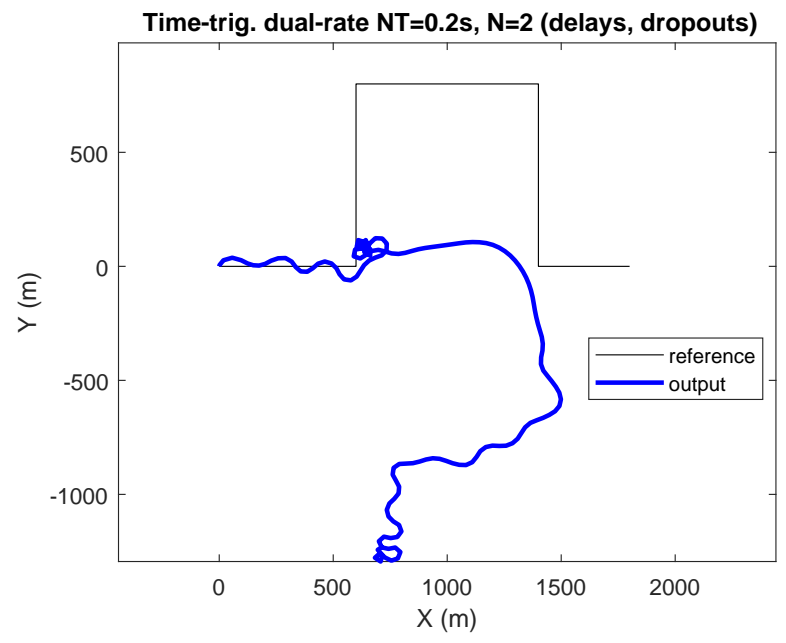

Figure 7.9: Time-triggered dual-rate control (no noise, no disturbance, delays, dropouts)

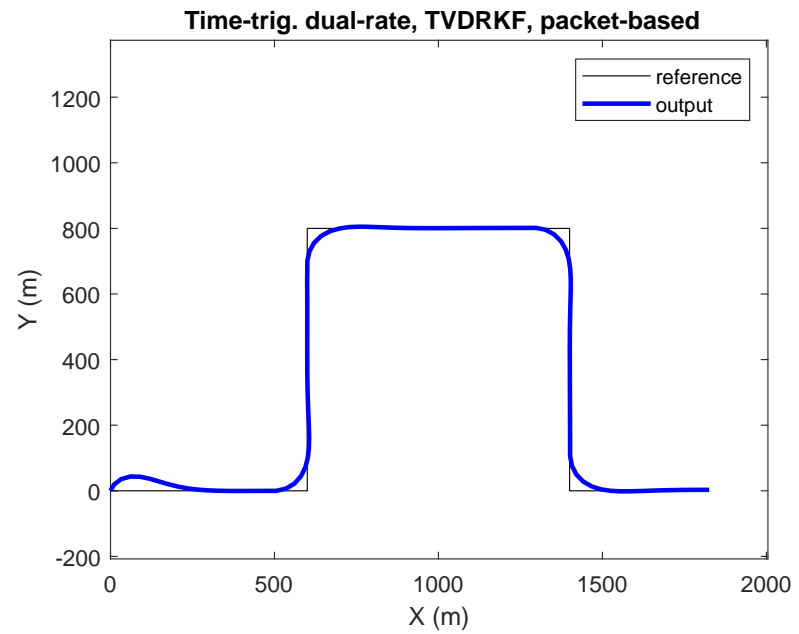

Figure 7.10: Time-triggered dual-rate control, with TVDRKF, and packet-based control (noise, disturbance, delays, dropouts)

Finally, event-triggered conditions are added to the WSN, and then, the system becomes a periodic event-triggered dual-rate control system. The performance obtained is similar to that reached by the time-triggered version of the system (see in Figure 7.12), but now a clear reduction of the number of transmit- 


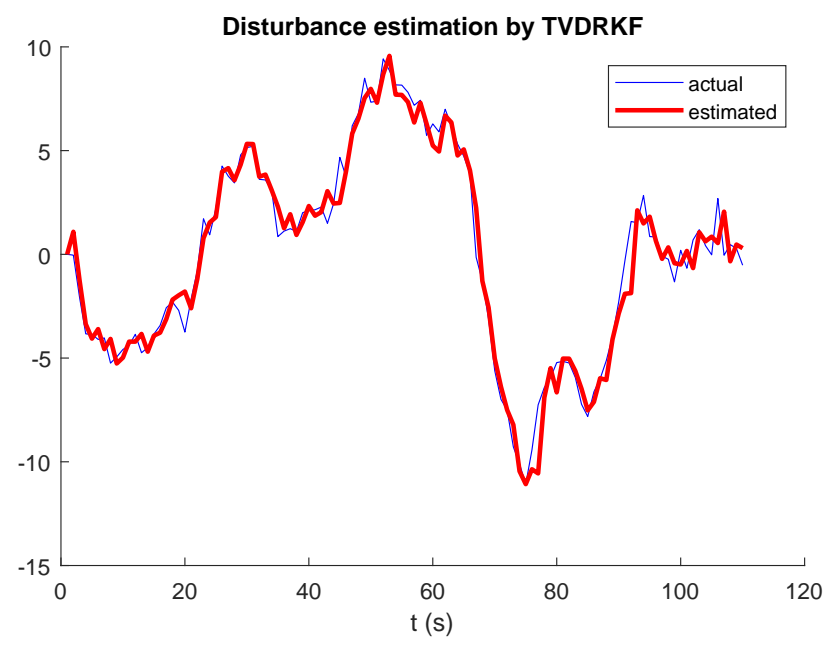

Figure 7.11: Disturbance estimation by TVDRKF

ted packets is achieved, which leads to reducing resource usage (bandwidth, energy).

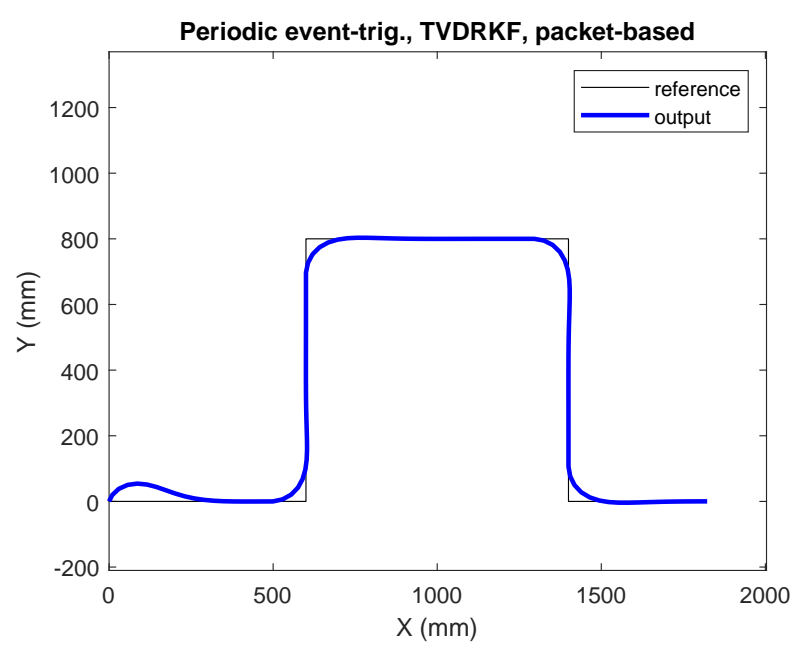

Figure 7.12: Periodic event-triggered dual-rate control, with TVDRKF, and packet-based control (noise, disturbance, delays, dropouts) 


\begin{tabular}{|l|lllll|}
\hline index & $a$ & $b$ & $c$ & $d$ & $e$ \\
\hline$J_{1}$ & 1671.8 & 1043.4 & 1029.9 & 1030.0 & 1184.4 \\
$J_{2}$ & 44.55 & 38.76 & 44.33 & 44.33 & 44.97 \\
$J_{3}$ & $22.4 \mathrm{~s}$ & $22.0 \mathrm{~s}$ & $22.0 \mathrm{~s}$ & $22.0 \mathrm{~s}$ & $22.0 \mathrm{~s}$ \\
$J_{4}$ & $50 \%$ & $100 \%$ & $50 \%$ & $48.18 \%$ & $26.13 \%$ \\
\hline
\end{tabular}

Table 7.1: Cost indexes

To analyze the previous conclusions in more detail, the cost indexes presented in section 7.4 are calculated for each scenario. Table 7.1 shows these results, where each scenario is represented by the following letters:

- $a$ : time-triggered single-rate control scenario at period $N T$.

- $b$ : time-triggered single-rate control scenario at period $T$.

- $c$ : time-triggered dual-rate control scenario.

- $d$ : time-triggered dual-rate control scenario, adding TVDRKF, and packetbased control.

- $e$ : periodic event-triggered dual-rate control scenario, with TVDRKF, and packet-based control.

As previously commented, the desired, nominal performance is presented by scenario $b$, and hence, $J_{1}, J_{2}, J_{3}$ show the reference values to carry out the comparison. As expected, scenario a shows the worst $J_{1}$, since the desired trajectory is inaccurately followed by the UGV. Scenarios $c$ and $d$ show a similar $J_{1}$ than scenario $b$ (even $1 \%$ better). Scenario $e$ worsens this index $J_{1}$ around $13 \%$. Regarding index $J_{2}$, it is worsened around $16 \%$ by every scenario compared to the nominal performance. However, $J_{3}$ presents the same value for cases $c, d$, and $e$, being $1 \%$ worse for scenario $a$. Finally, as expected, $J_{4}$ presents the worst case in scenario $b$, being $50 \%$ reduced by scenarios $a$ and $c$ because of sampling the network two times slower, and even a bit more than $50 \%$ of reduction (around 52\%) in scenario $d$, due to the additional packet dropouts. Scenario $e$ reaches the best value for index $J_{4}$ with around $75 \%$ of reduction.

As a summary, the proposed control approach is able to significantly reduce resource usage (around $75 \%$ ) while keeping satisfactory control properties, which is only worsened around $15 \%$ on average, and despite the existence of wireless communication problems such as time-varying delays and packet dropouts. 


\subsection{Conclusions}

The proposed energy-efficient control solution for a UGV in a WSN enables to lessen the amount of transmissions through the network, which results in bandwidth and battery saving, despite keeping a satisfactory system performance. The solution integrates dual-rate control, periodic event-triggered control, packed-based control, and time-varying dual-rate Kalman filter based prediction techniques. Additionally, the approach deals with wireless communication problems (such as time-varying delays, packet dropouts, and packet disorder) and copes with a realistic scenario, where measurement noise and external disturbance can occur.

\section{Acknowledgements}

This research work has been developed as a result of a mobility stay funded by the Spain Visiting Fulbright Scholar Programme of the Fulbright Commission and the Spanish Ministry of Education under "Programa Estatal de Promoción del Talento y su Empleabilidad en $\mathrm{I}+\mathrm{D}+\mathrm{i}$, Subprograma Estatal de Movilidad, del Plan Estatal de Investigación Científica y Técnica y de Innovación 2013$2016 "$.

In addition, the work is funded by European Commission as part of Project H2020-SEC-2016-2017 - Topic: SEC-20-BES-2016 - Id: 740736 - "C2 Advanced Multi-domain Environment and Live Observation Technologies" (CAMELOT). Part WP5 supported by Tekever ASDS, Thales Research and Technology, Viasat Antenna Systems, Universitat Politècnica de València, Fundação da Faculdade de Ciências da Universidade de Lisboa, Ministério da Defensa Nacional - Marinha Portuguesa, Ministério da Administração Interna Guarda Nacional Republicana. 


\section{References}

[1] Jennifer Yick, Biswanath Mukherjee, and Dipak Ghosal. "Wireless sensor network survey". In: Computer Networks 52.12 (2008), pp. 2292-2330. ISSN: 1389-1286. DOI: https ://doi .org/10.1016/j . comnet . 2008.04 . 002 (cit. on p. 202).

[2] Ian F Akyildiz et al. "Wireless sensor networks: a survey". In: Computer Networks 38.4 (2002), pp. 393-422. ISSN: 1389-1286. DOI: https://doi. org/10.1016/S1389-1286(01)00302-4 (cit. on p. 202).

[3] Asmaa Ez-Zaidi and Said Rakrak. "A Comparative Study of Target Tracking Approaches in Wireless Sensor Networks". In: Journal of Sensors 2016 (Jan. 2016), pp. 1-11. DOI: 10.1155/2016/3270659 (cit. on p. 202).

[4] Asmaa Ez-zaidi and Said Rakrak. "Energy efficient approach for target tracking in Wireless Sensor Networks". In: Int. J. Computer Scie. Network Security 17.9 (2017), pp. 95-101 (cit. on p. 202).

[5] Ping Wang et al. "Investigation of wireless sensor networks for structural health monitoring". In: Journal of Sensors 2012 (2012) (cit. on p. 202).

[6] Adam B Noel et al. "Structural Health Monitoring Using Wireless Sensor Networks: A Comprehensive Survey". In: IEEE Communications Surveys Tutorials 19.3 (Apr. 2017), pp. 1403-1423. ISSN: 1553-877X. DOI: 10. 1109/COMST . 2017.2691551 (cit. on p. 202).

[7] Gongbo Zhou et al. "Harvesting ambient environmental energy for wireless sensor networks: a survey". In: Journal of Sensors 2014 (2014) (cit. on p. 202).

[8] P Visconti et al. "Development and Characterization of a solar-based energy harvesting and power management system for a WSN node applied to optimized goods transport and storage". In: Int. J. Smart Sensing and Intelligent Systems 9.4 (2016), pp. 1637-1667 (cit. on p. 202). 
[9] V. Raghunathan et al. "Energy-aware wireless microsensor networks". In: IEEE Signal Processing Magazine 19.2 (Mar. 2002), pp. 40-50. ISSN: 1053-5888. DOI: 10.1109/79.985679 (cit. on p. 202).

[10] Giuseppe Anastasi et al. "Energy conservation in wireless sensor networks: A survey". In: Ad Hoc Networks 7.3 (2009), pp. 537-568. ISSN: 1570-8705. DOI: https : //doi .org/10.1016/j . adhoc . 2008.06.003 (cit. on p. 202).

[11] Venkatesh Rajendran, Katia Obraczka, and Jose Joaquin Garcia-LunaAceves. "Energy-Efficient, Collision-Free Medium Access Control for Wireless Sensor Networks". In: Wireless Networks 12.1 (Feb. 2006), pp. 63-78. ISSN: 1572-8196. DOI: 10.1007/s11276-006-6151-z (cit. on p. 202).

[12] Yanjun Sun, Omer Gurewitz, and David B Johnson. "RI-MAC: a receiverinitiated asynchronous duty cycle MAC protocol for dynamic traffic loads in wireless sensor networks". In: Proc. 6th ACM Conf. Embedded network sensor systems. ACM. 2008, pp. 1-14 (cit. on p. 202).

[13] Q. Liu et al. "Event-Based Recursive Distributed Filtering Over Wireless Sensor Networks". In: IEEE Transactions on Automatic Control 60.9 (Sept. 2015), pp. 2470-2475. ISSN: 0018-9286. DOI: 10.1109/TAC. 2015. 2390554 (cit. on pp. 202, 203).

[14] Xiaohua Ge, Qing-Long Han, and Zidong Wang. "A threshold-parameterdependent approach to designing distributed event-triggered $H_{\infty}$ consensus filters over sensor networks". In: IEEE Trans. Cybern. (2018). DOI: 10.1109/TCYB. 2017.2789296 (cit. on pp. 202, 203).

[15] Noor Zaman, Low Tang Jung, and Muhammad Mehboob Yasin. "Enhancing energy efficiency of wireless sensor network through the design of energy efficient routing protocol". In: Journal of Sensors 2016 (2016) (cit. on p. 202).

[16] Mario Di Francesco, Sajal K. Das, and Giuseppe Anastasi. "Data Collection in Wireless Sensor Networks with Mobile Elements: A Survey". In: ACM Trans. Sen. Netw. 8.1 (Aug. 2011), 7:1-7:31. ISSN: 1550-4859. DOI: 10.1145/1993042.1993049 (cit. on p. 202).

[17] W. P. M. H. Heemels, M. C. F. Donkers, and A. R. Teel. "Periodic EventTriggered Control for Linear Systems". In: IEEE Transactions on Automatic Control 58.4 (Apr. 2013), pp. 847-861. ISSN: 0018-9286. DOI: 10.1109/TAC.2012.2220443 (cit. on p. 203).

[18] W. P. M. H. Heemels and M. C. F. Donkers. "Model-based periodic eventtriggered control for linear systems". In: Automatica 49.3 (2013), pp. 698711. ISSN: 0005-1098. DOI: https://doi.org/10.1016/j . automatica. 2012.11.025 (cit. on p. 203). 
[19] Á. Cuenca et al. "Periodic Event-Triggered Sampling and Dual-Rate Control for a Wireless Networked Control System With Applications to UAVs". In: IEEE Transactions on Industrial Electronics 66.4 (Apr. 2019), pp. 3157-3166. ISSN: 0278-0046. DOI: 10.1109/TIE. 2018.2850018 (cit. on p. 203).

[20] C. Peng, D. Yue, and M. Fei. "A Higher Energy-Efficient Sampling Scheme for Networked Control Systems over IEEE 802.15.4 Wireless Networks". In: IEEE Transactions on Industrial Informatics 12.5 (Oct. 2016), pp. 17661774. ISSN: 1551-3203. DOI: 10.1109/TII .2015.2481821 (cit. on p. 203).

[21] Jan Lunze and Daniel Lehmann. "A state-feedback approach to eventbased control". In: Automatica 46.1 (2010), pp. 211-215. ISSN: 0005-1098. DOI: https://doi.org/10.1016/j.automatica.2009.10.035 (cit. on p. 203).

[22] Xiaohua Ge and Qing-Long Han. "Distributed event-triggered $H_{\infty}$ filtering over sensor networks with communication delays". In: Information Sciences 291 (2015), pp. 128-142. ISSN: 0020-0255. DOI: https : //doi.org/10.1016/j.ins.2014.08.047 (cit. on p. 203).

[23] Eloy Garcia and Panos J Antsaklis. "Model-Based Event-Triggered Control for Systems With Quantization and Time-Varying Network Delays". In: IEEE Transactions on Automatic Control 58.2 (Feb. 2013), pp. 422434. ISSN: 0018-9286. DOI: 10.1109/TAC.2012.2211411 (cit. on p. 203).

[24] Roy McCann and Anh T Le. "Lebesgue Sampling with a Kalman Filter in Wireless Sensors for Smart Appliance Networks". In: 2008 IEEE Industry Applications Society Annual Meeting. Oct. 2008, pp. 1-5. DOI: 10.1109/ 08IAS. 2008.9 (cit. on pp. 203, 213).

[25] S. Trimpe and R. D'Andrea. "Event-Based State Estimation With VarianceBased Triggering". In: IEEE Transactions on Automatic Control 59.12 (Dec. 2014), pp. 3266-3281. ISSN: 0018-9286. DOI: 10.1109/TAC. 2014. 2351951 (cit. on pp. 203, 213).

[26] Ángel Cuenca et al. "Non-uniform Multi-rate Estimator based Periodic Event-Triggered Control for resource saving". In: Information Sciences 459 (2018), pp. 86-102. ISSN: 0020-0255. DOI: https ://doi .org/10. 1016/j.ins.2018.05.038 (cit. on pp. 203, 213).

[27] Yun-Bo Zhao, Guo-Ping Liu, and David Rees. "Design of a Packet-Based Control Framework for Networked Control Systems". In: IEEE Transactions on Control Systems Technology 17.4 (July 2009), pp. 859-865. ISSN: 1063-6536. DOI: 10.1109/TCST . 2008. 2010946 (cit. on p. 203). 
[28] Yun-Bo Zhao et al. "Packet-Based Control Design for Networked Control Systems". In: Packet-Based Control for Networked Control Systems. Springer, 2018, pp. 15-32 (cit. on p. 203).

[29] Julián Salt and Pedro Albertos. "Model-based multirate controllers design". In: IEEE Transactions on Control Systems Technology 13.6 (Nov. 2005), pp. 988-997. ISSN: 1063-6536. DOI: 10.1109/TCST . 2005.857410 (cit. on pp. 204, 220-222).

[30] Julián Salt et al. "A Multirate Control Strategy to the Slow Sensors Problem: An Interactive Simulation Tool for Controller Assisted Design". In: Sensors 14.3 (2014), pp. 4086-4110. ISSN: 1424-8220. DOI: 10.3390/ s140304086 (cit. on pp. 204, 205, 220).

[31] Wei Chen and Li Qiu. "Stabilization of networked control systems with multirate sampling". In: Automatica 49.6 (2013), pp. 1528-1537. ISSN: 0005-1098. DOI: https://doi .org/10.1016/j . automatica. 2013.02 . 010 (cit. on p. 204).

[32] Jean-Chrysotome Bolot. "End-to-end packet delay and loss behavior in the Internet". In: ACM SIGCOMM Computer Communication Review. Vol. 23. 4. ACM. 1993, pp. 289-298 (cit. on pp. 204, 207).

[33] Kenzo Nonami et al. "Autonomous control systems and vehicles". In: Intelligent Systems, Control and Automation: Science and Engineering 65 (2013) (cit. on p. 204).

[34] Barbara Arbanas et al. "Decentralized planning and control for UAVUGV cooperative teams". In: Autonomous Robots 42.8 (Dec. 2018), pp. 16011618. ISSN: 1573-7527. DOI: 10.1007 / s10514-018-9712-y (cit. on p. 204).

[35] Thomas Hellstrom and Ola Ringdahl. "Follow the Past: a path-tracking algorithm for autonomous vehicles". In: International journal of vehicle autonomous systems 4.2 (2006), pp. 216-224 (cit. on p. 204).

[36] Zirui Li et al. "Development and Evaluation of Two Learning-Based Personalized Driver Models for Pure Pursuit Path-Tracking Behaviors". In: 2018 IEEE Intelligent Vehicles Symposium (IV). June 2018, pp. 79-84. DOI: $10.1109 /$ IVS.2018.8500618 (cit. on p. 204).

[37] A. Cervin et al. "How does control timing affect performance? Analysis and simulation of timing using Jitterbug and TrueTime". In: IEEE Control Systems Magazine 23.3 (June 2003), pp. 16-30. ISSN: 1066-033X. DOI: 10.1109/MCS.2003.1200240 (cit. on pp. 205, 223). 
[38] T. Cooklev, J. C. Eidson, and A. Pakdaman. "An Implementation of IEEE 1588 Over IEEE 802.11b for Synchronization of Wireless Local Area Network Nodes". In: IEEE Transactions on Instrumentation and Measurement 56.5 (Oct. 2007), pp. 1632-1639. ISSN: 0018-9456. DOI: 10. 1109/TIM. 2007.903640 (cit. on p. 206).

[39] Y. Tipsuwan and Mo-Yuen Chow. "Gain scheduler middleware: a methodology to enable existing controllers for networked control and teleoperation - part I: networked control". In: IEEE Transactions on Industrial Electronics 51.6 (Dec. 2004), pp. 1218-1227. ISSN: 0278-0046. DOI: 10.1109/TIE. 2004.837866 (cit. on p. 207).

[40] Xian-Ming Zhang, Qing-Long Han, and Xinghuo Yu. "Survey on Recent Advances in Networked Control Systems". In: IEEE Transactions on Industrial Informatics 12.5 (Oct. 2016), pp. 1740-1752. ISSN: 1551-3203. DOI: 10.1109/TII.2015.2506545 (cit. on p. 207).

[41] P. Khargonekar, K. Poolla, and A. Tannenbaum. "Robust control of linear time-invariant plants using periodic compensation". In: IEEE Transactions on Automatic Control 30.11 (Nov. 1985), pp. 1088-1096. ISSN: 0018-9286. DOI: 10.1109/TAC.1985.1103841 (cit. on p. 211).

[42] D. P. (. Borgers and W. P. M. H. (. Heemels. "Event-Separation Properties of Event-Triggered Control Systems". In: IEEE Transactions on Automatic Control 59.10 (Oct. 2014), pp. 2644-2656. ISSN: 0018-9286. DOI: $10.1109 /$ TAC.2014.2325272 (cit. on p. 212).

[43] L. Xing et al. "Event-Triggered Adaptive Control for a Class of Uncertain Nonlinear Systems". In: IEEE Transactions on Automatic Control 62.4 (Apr. 2017), pp. 2071-2076. ISSN: 0018-9286. DOI: 10.1109/TAC. 2016. 2594204 (cit. on p. 212).

[44] Dan Simon. Optimal state estimation: Kalman, $H_{\infty}$, and nonlinear approaches. John Wiley \& Sons, 2006. ISBN: 9780470045336 (cit. on p. 213).

[45] Minghui Zheng, Liting Sun, and Masayoshi Tomizuka. "Multi-rate Observer Based Sliding Mode Control with Frequency Shaping for Vibration Suppression Beyond Nyquist Frequency**This work was sponsored by Western Digital Corporation." In: IFAC-PapersOnLine 49.21 (2016). 7th IFAC Symposium on Mechatronic Systems MECHATRONICS 2016, pp. 13-18. ISSN: 2405-8963. DOI: https://doi.org/10.1016/j.ifacol. 2016.10.504 (cit. on p. 213).

[46] Minghui Zheng, Xu Chen, and Masayoshi Tomizuka. "Extended state observer with phase compensation to estimate and suppress high-frequency disturbances". In: 2016 American Control Conference (ACC). July 2016, pp. 3521-3526. DOI: 10.1109/ACC. 2016.7525459 (cit. on p. 213). 
[47] Andrew Smyth and Meiliang Wu. "Multi-rate Kalman filtering for the data fusion of displacement and acceleration response measurements in dynamic system monitoring". In: Mechanical Systems and Signal Processing 21.2 (2007), pp. 706-723. ISSN: 0888-3270. DOI: https://doi.org/ 10.1016/j.ymssp.2006.03.005 (cit. on p. 213).

[48] Martin Lundgren. "Path tracking and obstacle avoidance for a miniature robot". In: Umeå University, Umeå, Master Thesis (2003) (cit. on p. 216).

[49] Ángel Cuenca et al. "A non-uniform multi-rate control strategy for a Markov chain-driven Networked Control System". In: Information Sciences 321 (2015). Security and privacy information technologies and applications for wireless pervasive computing environments, pp. 31-47. ISSN: 0020-0255. DOI: https://doi.org/10.1016/j.ins .2015.05.035 (cit. on pp. 222, 223).

[50] Katsuhiko Ogata. Discrete-time control systems. Vol. 2. Prentice Hall Englewood Cliffs, NJ, 1995 (cit. on p. 224).

[51] Karl J Aström and Tore Hägglund. "PID controllers: theory, design, and tuning". In: Instrument Society of America 67 (1995) (cit. on p. 224). 


\title{
Chapter 8
}

\section{Paper 7. A remote control strategy for an au- tonomous vehicle with slow sensor using Kalman filtering and dual-rate control}

\author{
Ángel Cuenca, Wei Zhan, Julián Salt, José Alcaina, Chen \\ Tang, Masayoshi Tomizuka
}

This work presents a novel remote control solution for an Autonomous Vehicle $(A V)$, where the system structure is split into two sides. Both sides are assumed to be synchronized and linked through a communication network, which introduces time-varying delays and packet disorder. An Extended Kalman Filter (EKF) is used to cope with the non-linearities that appear in the global model of the AV. The EKF fuses the data provided by the sensing devices of the $A V$ in order to estimate the AV state, reducing the noise effect. Additionally, the EKF includes an h-step-ahead state prediction stage, which, together with the consideration of a packet-based control strategy, enables facing the network-induced delays. Since the $A V$ position is provided by a camera, which is a slow sensing device, a dual-rate controller is required to achieve certain desired (nominal) dynamic control performance. The use of a dual-rate control framework additionally enables saving network bandwidth and deals with packet disorder. As the path-tracking control algorithm, pure pursuit is used. Application results show that, despite existing communication problems and slow-rate measurements, the $A V$ is able to track the desired path, keeping the nominal control performance.

Keywords: Autonomous vehicle; slow sensor; Kalman filter; networked control; dual-rate control.

Sensors, volume 19 - issue 13, jul 2019, page 2983, https: // doi. org/10. 3390/s19132983 


\subsection{Introduction}

In this work, a remote control solution is proposed for Autonomous Vehicle (AV) path tracking. The solution fits in the field of Networked Control Systems (NCSs) [1-4], which is a prolific control area that addresses control scenarios where different devices share a common communication link. The use of a shared communication network introduces some advantages like cost reduction, reconfigurability, and ease of installation and maintenance, but also some drawbacks such as the possible occurrence of network-induced delays [510], packet dropouts [11-13], packet disorder [14-16], and network bandwidth constraints [17-19]. In this work, the drawbacks considered in the network are time-varying delays and packet disorder.

An AV can be defined as a vehicle that is capable of intelligent motion and action without human input [20]. AVs have attracted the attention of the scientific community due to the large number of applications in which they can be involved (for instance, target tracking [21], surveillance [22], transportation [23], self-driving cars [24], etc.). In our work, a target tracking application is developed, where the AV concretely is a differential robot. As the path tracking algorithm, pure pursuit [25-27] has been chosen. The hierarchical structure of the AV (see, e.g., [28]) is split into two sides, with the communication network between them. In particular, the motion planning and vehicle control layers are located on-board, whereas upper levels of the structure such as decisionmaking, path generation, and monitoring are located at the remote server. In this way, safety-critical tasks are executed on-board in real-time, whereas longer term computations, which mostly affect performance and are generally not real-time critical, are executed in the remote server.

The present work is mainly focused on designing a control solution at the local side, which will integrate state estimation, packet-based control, and dual-rate control. The main idea is to generate a set of path references from way points at the remote server, and send them to the AV through the network. Then, the control level is able to compute a delay-free control signal to be applied to the $\mathrm{AV}$ and generate a set of future state estimations, which is sent to the remote server for upper level purposes such as displaying variables, making decisions (e.g., trajectory replanning), etc.

As is well known, AV path tracking involves non-linearities [29]. Sensed variables must be accurately estimated and corrected in order to provide the motion planning and control layers with reliable data. For this purpose, different filtering approaches can be used, such as the Kalman filter (see, for example, [30-32]) and the $H_{\infty}$ filter (see, for instance, [32, 33]), among others. The 
attractiveness of the Kalman filter lies in the fact that it is the one estimator that results in the smallest possible standard deviation of the estimator error. However, when the goal is to minimize the worst-case estimation error, the $H_{\infty}$ filter is a better option. The $H_{\infty}$ filter provides a rigorous method for dealing with systems that have model uncertainty, but is more sensitive to the design parameters (weighting functions) than the Kalman filter. As a conclusion, $H_{\infty}$ theory shows us the optimal way to robustify the Kalman filter, this theory being more abstract and complicated than the one underlying the Kalman filtering.

Focusing on the Kalman filter, it is widely used in its extended and unscented versions (see, e.g., [29-31, 34]). In this work, the Extended Kalman Filter (EKF) has been chosen not only to estimate the non-linear behavior of the $\mathrm{AV}$, reducing the process and measurement noise effect, but also to fuse all the data provided by the different sensors in order to be used by the control algorithm. The sensing devices used in our path tracking application are two encoders, an Inertial Measurement Unit (IMU) and a zenithal camera, which respectively provide the angular velocities of each wheel, the AV orientation, and the AV position. In our proposal, the EKF is complemented with an $h$-step-ahead state prediction stage, which, together with the integration of packet-based control strategies in the control solution, enables compensating for time-varying network-induced delays.

Packet-based control $[35,36]$ is usually employed to decrease the communication rate by simultaneously sending a set of data in each transmission. In our work, the main aim of including this technique in the control solution is two-fold:

1. To provide the motion planning and control layers with a set of $h$-stepahead path references. From this set and after successively iterating the different components of these layers in the current sensing period, a set of $h$-step-ahead control action estimates can be computed by following a delay-free control algorithm.

2. To supply the upper layers of the AV structure included at the remote server with a set of $h$-step-ahead state predictions (computed by the motion planning and control layers in every sensing period), which is updated irrespective of the delay when a new packet is received.

Then, for implementation purposes, the time-varying network-induced delay is not required to be measured and compensated for. This is a relevant aspect of this work, since it makes the solution applicable to a wide range of wireless 
control applications where the time delay is difficult to measure. This working mode is possible because an accurate system model and an acceptable level of noise are assumed, and hence, the difference between actual and estimated signals should be negligible. In addition, the possible divergences between both signals can be corrected by the EKF.

In dual-rate control [37-40], a slower sensing rate in comparison to a faster actuation can be assumed. As mentioned, in this work, a zenithal camera is used to sense the AV position, which is provided at a slow rate due to technical constraints. Despite sensing in this way, the fast actuation enables achieving acceptable control properties. In addition, dual-rate control techniques provide two-fold benefits: (i) to reduce the amount of transmissions through the network, which results in bandwidth saving; (ii) to elude packet disorder, selecting the sampling period to be larger than the largest network-induced time delay. As a statistical distribution for the delay is assumed to be known [41], the largest delay can be easily found. In the present work, due to the broad knowledge of PID controllers in academic and industrial environments, a dual-rate PID control scheme is considered.

In summary, the main contribution of the present work is the development of a novel and complete remote control approach for an AV, where an EKF (including an $h$-step-ahead state prediction stage), packet-based control, the pure pursuit path tracking algorithm, and a dual-rate dynamic controller are systematically brought together in order to keep the nominal control properties when path tracking and considerably lessen resource usage such as network bandwidth. These facts are reached despite existing wireless communication problems such as time-varying delays and packet disorder, and the appearing process and measurement noise signals. The nominal control performance is defined by means of an ideal fast-rate control framework, where no noise and delay are considered.

In section 8.2, the novel control structure is presented. In addition, some details about how the network-induced delays can be faced are posed. In section 8.3, the non-linear model of the AV is introduced, and then, the control design of the motion planning and control layers of the AV structure is formulated. section 8.4 presents some cost indexes to measure and compare control performance among the proposed control solution and other approaches. section 8.5 shows the main benefits of the control proposal by means of a Truetime application [42]. Finally, section 8.6 summarizes the main conclusions of the work. 


\subsection{Problem Scenario}

The overall control scheme is depicted in Figure 8.1. The proposed structure includes two sides: (i) the local side, where the AV is located together with different actuators and sensors (which can introduce measurement noise) and where motion planning and vehicle control layers are implemented; and (ii) the remote side, where upper level layers of the hierarchical structure of the AV are included, in such a way that the remote server is in charge of generating the path reference based on way points, displaying and monitoring system data, and making decisions from this information. A wireless network is used to connect both sides, which introduces some communication problems such as time-varying delays and packet disorder. In the next subsections, these problems are formally described, and the working mode of the control structure is presented.

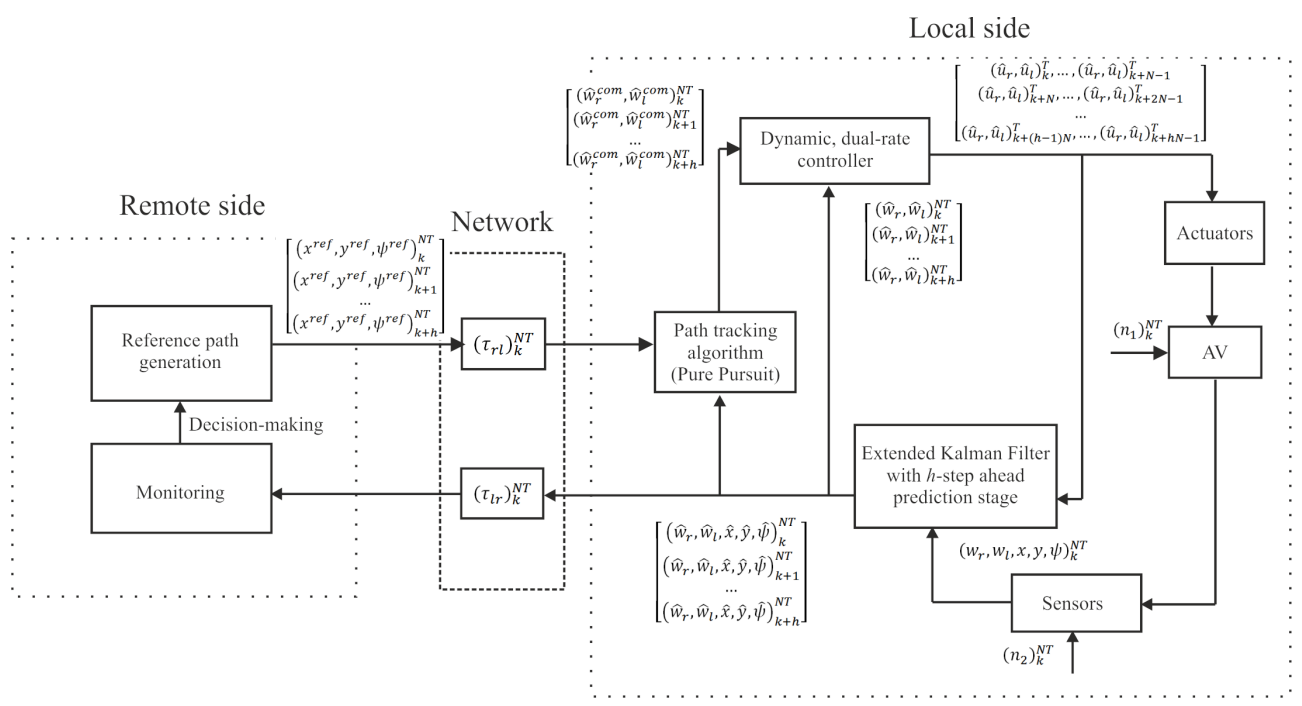

Figure 8.1: Remote control structure for an autonomous vehicle

\subsubsection{Control Structure}

There are three main keys to the control solution:

1. Fusing all the data provided by the different sensors (encoders, IMU, camera) by means of an Extended Kalman Filter (EKF) in order to estimate 
the state of the AV, reducing the noise effect. The extended version of the Kalman Filter is needed due to the non-linear nature of the AV.

2. Including an $h$-step-ahead state prediction stage in the EKF, using a packet-based control strategy, for the purpose of dealing with networkinduced delays, and providing the remote side with future, estimated data.

3. Integrating dual-rate control with a view toward achieving the desired (nominal) control specifications, coping with slow sensing and packet disorder.

The control structure uses two different periods: $T$ as the actuation period and $N T$ as the sensing period, $N \in \mathbb{N}^{+}$being the multiplicity between the two periods of the dual-rate control scheme [38]. Let us respectively denote $(.)_{k}^{T}$ and $(.)_{k}^{N T}$ as a $T$-period and an $N T$-period signal or variable, where $k \in \mathbb{N}$ are iterations in the corresponding period. Communication between the two network sides is carried out in the sensing period $N T$, i.e., at instants $k N T$, which helps save network bandwidth; concretely, the use of the network is $\mathrm{N}$ times lower than that produced by a conventional single-rate control in period $T$.

In more detail, the control structure works as follows:

- At the current instant $k N T$, the remote side generates a set of $h$ path references, which includes from the reference to be used at instant $k$, i.e., $\left(x^{r e f}, y^{r e f}, \psi^{r e f}\right)_{k}^{N T}$, to the reference to be used at instant $(k+h) N T$, i.e., $\left(x^{r e f}, y^{r e f}, \psi^{r e f}\right)_{k+h}^{N T}$. The set is composed of reference positions $\left(x^{r e f}, y^{r e f}\right)$ and reference yaw angle $\psi^{r e f}$, and it is sent to the local side in a packet.

- The local side gets the current system state $\left(w_{r}, w_{l}, x, y, \psi\right)_{k}^{N T}$, which coincides with the system output in this work, being affected by the process and measurement noises, $\left(n_{1}\right)_{k}^{N T}$ and $\left(n_{2}\right)_{k}^{N T}$, respectively. The state is composed of angular velocities $\left(w_{r}, w_{l}\right)$, positions $(x, y)$, and yaw angle $\psi$.

- $\quad$ The next estimation of the system state $\left(\hat{w}_{r}, \hat{w}_{l}, \hat{x}, \hat{y}, \hat{\psi}\right)_{k}^{N T}$ is computed via an Extended Kalman Filter (EKF). This estimation carried out by the EKF is actually the correction of the state.

- $\quad$ From this state and the path reference for the instant $k N T$, i.e., $\left(x^{\text {ref }}, y^{\text {ref }}\right.$ ,$\left.\psi^{r e f}\right)_{k}^{N T}$, received in the previous packet after the remote-to-local delay 
$\left(\tau_{r l}\right)_{k}^{N T}$, the path tracking algorithm (pure pursuit in this case) computes the dynamic reference, or command, for the instant $k N T$, i.e., $\left(\hat{w}_{r}^{\text {com }}\right.$, $\left.\hat{w}_{l}^{\text {com }}\right)_{k}^{N T}$.

- From this dynamic reference and the estimated angular velocities $\left(\hat{w}_{r}\right.$, $\left.\hat{w}_{l}\right)_{k}^{N T}$, the dynamic, dual-rate controller computes the control signal to be applied to the AV, i.e., $\left\{\left(\hat{u}_{r}, \hat{u}_{l}\right)_{k}^{T}, \cdots,\left(\hat{u}_{r}, \hat{u}_{l}\right)_{k+N-1}^{T}\right\}$, which are the control actions in period $T$ for the right and left motors, respectively, inside the sensing period $k N T$. As a uniform actuation pattern, the actuation occurs at uniformly-spaced instants $k N T+l T(l=0,1, \cdots, N-1)$ under Zero Order Hold $(\mathrm{ZOH})$ conditions inside the sensing period. That is, $\left(\hat{u}_{r}, \hat{u}_{l}\right)_{k}^{T}$ is applied at $k N T,\left(\hat{u}_{r}, \hat{u}_{l}\right)_{k+1}^{T}$ is injected at $k N T+T$, and so on, up to $\left(\hat{u}_{r}, \hat{u}_{l}\right)_{k+N-1}^{T}$, which is actuated at $k N T+(N-1) T$.

- From this control signal and the estimated state for the instant $k N T$, the $h$-step-ahead state prediction stage is able to compute the estimation of the state for the instant $(k+1) N T$, i.e., $\left(\hat{w}_{r}, \hat{w}_{l}, \hat{x}, \hat{y}, \hat{\psi}\right)_{k+1}^{N T}$. This computation is carried out in an open loop. Iterating the control loop $h$ times, the local side can obtain the $h$ state estimations to be sent in a packet to the remote side. Therefore, when the remote server receives the packet at the current time instant $k N T$ and after the local-to-remote delay $\left(\tau_{l r}\right)_{k}^{N T}$, it can manage future system information, for example, to be displayed and to make decisions.

\subsubsection{Time-Varying Delays, and Packet Disorder}

Assuming synchronization between both network sides [43], time-varying delays can be measured for both communication links. From off-line experiences on the communication network, a statistical distribution of the delay can be obtained and, hence, the maximum delay $\tau_{\max }$. Packet disorder may appear when $N T<\tau_{\max }$. In the present work, by taking advantage of the use of dual-rate control to deal with slow sensing, a straightforward solution is considered to avoid packet disorder, that is setting up the sampling period $N T$ to be larger than the maximum time delay $\tau_{\max }$, i.e., $N T>\tau_{\max }$.

In order to cope with time-varying delays, the control solution integrates a packet-based strategy and predictor-based control. Next, details about how the delays can be faced are given:

- Remote-to-local delays $\left(\tau_{r l}\right)_{k}^{N T}$ : By implementing the packet-based strategy, the packet with the set of references generated by the remote server from the instant $k N T$ to the instant $(k+h) N T$ arrives after the delay 


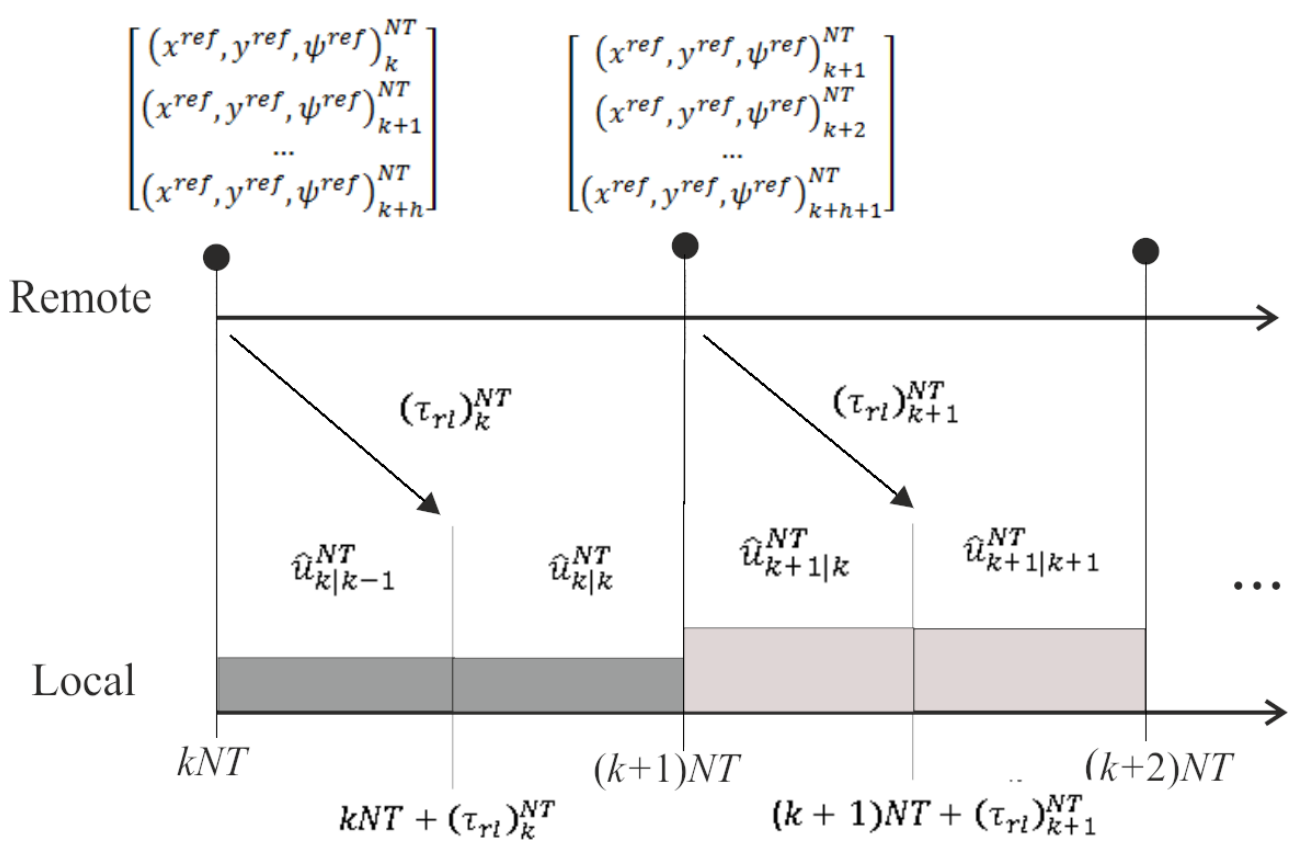

Figure 8.2: Time axis example for the remote-to-local channel

$\left(\tau_{r l}\right)_{k}^{N T}$ at the local side. As a consequence of integrating predictor-based control and assuming an accurate system model and an acceptable level of noise, this delay will not affect the control system. The reason is that, since the reference for the current instant was received in the previous delivery, the consequent estimated control action is already being injected from the beginning of the current period. When the packet is received, a new control action is computed from the corrected state. This control action will be very similar to the estimated one, and hence, an insignificant change is produced in the control signal. Note that possible changes in the reference due to decision-making tasks are recommended to be included at least from instant $(k+1) N T$ of the set of references in order to keep the described working mode, avoiding the delay effect. Figure 8.2 depicts a time axis example of this communication channel, where, for the sake of simplicity, a single-rate control at NT is considered. Notation $\hat{u}_{k+1 \mid k}^{N T}$ means the estimated control action to be applied at instant $(k+1) N T$, which is calculated at instant $k N T$ from the reference $\left(x^{r e f}, y^{r e f}, \psi^{r e f}\right)_{k+1}^{N T}$. As shown, when the packet is received, a new control action is computed, which is practically the same as that pre- 


$\left.\begin{array}{|c|c|}\hline\left[\begin{array}{c}\hat{\xi}_{k \mid k}^{N T} \\ \hline \hline \hat{\xi}_{k+1 \mid k}^{N T} \\ \cdots \\ \hat{\xi}_{k+h \mid k}^{N T}\end{array}\right] & {\left[\hat{\xi}_{k+1 \mid k+1}^{N T}\right.} \\ \end{array}\right]$

corrected by EKF

estimated by prediction stage

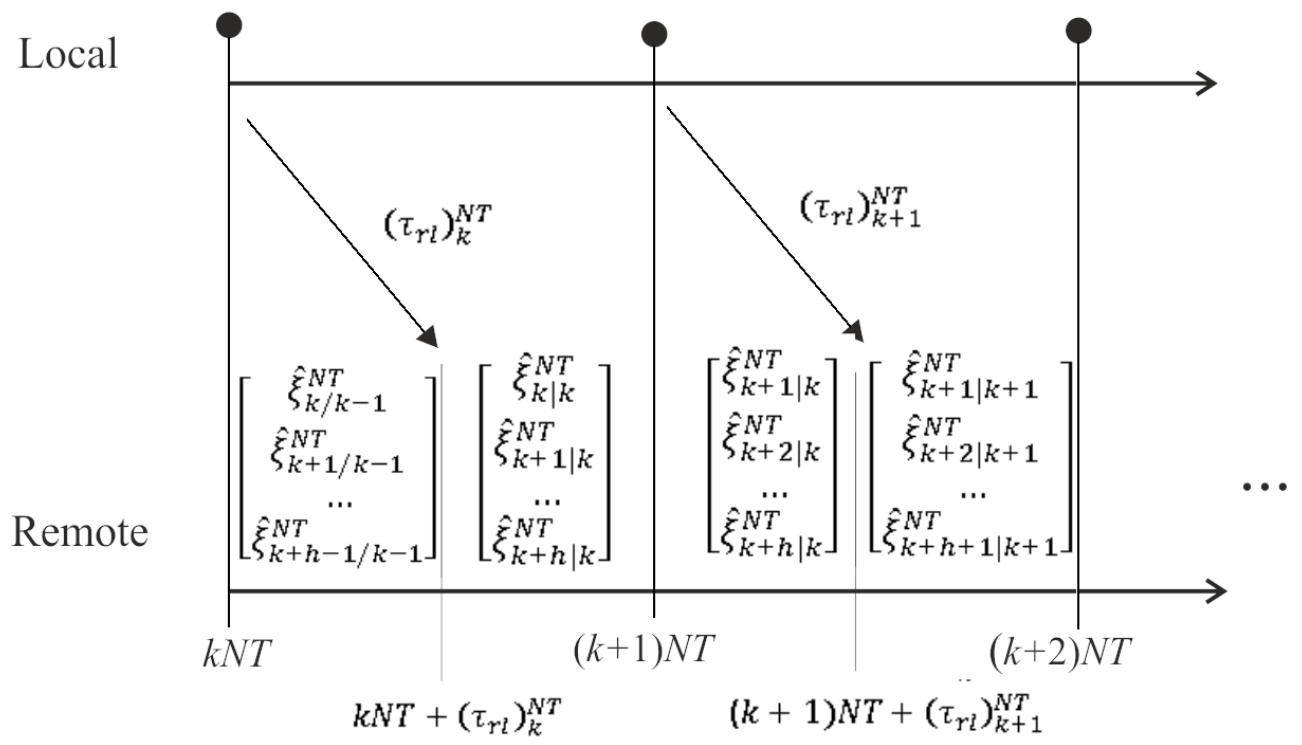

Figure 8.3: Time axis example for the local-to-remote channel

viously calculated from the preceding delivery, i.e., $\hat{u}_{k / k-1}^{N T} \cong \hat{u}_{k / k}^{N T}$ and $\hat{u}_{k+1 / k}^{N T} \cong \hat{u}_{k+1 / k+1}^{N T}$.

- Local-to-remote delays $\left(\tau_{l r}\right)_{k}^{N T}$ : Similar to the other link, by implementing the packet-based strategy, the packet with the set of estimations generated by the EKF and the $h$-step-ahead state prediction stage from instant $k N T$ to instant $(k+h) N T$ arrives after the delay $\left(\tau_{l r}\right)_{k}^{N T}$ at the remote side. The working mode is as follows: from the beginning of the period, a set of state estimates is used, and when a new packet arrives, this set is replaced with the received one, which includes state correction. Figure 8.3 depicts a time axis example of this communication channel. The notation $\hat{\xi}_{k+1 \mid k}^{N T}$ means the state estimated for the instant $(k+1) N T$ at the instant $k N T$, i.e., $\left(\hat{w}_{r}, \hat{w}_{l}, \hat{x}, \hat{y}, \hat{\psi}\right)_{k+1 \mid k}^{N T}$. The estimation $\hat{\xi}_{k \mid k}^{N T}$ is 
actually the state corrected by the EKF, and hence, the state estimate $\hat{\xi}_{k \mid k-1}^{N T}$ can be replaced with its correction $\hat{\xi}_{k \mid k}^{N T}$. From this correction, the $h$-step-ahead state prediction stage estimates the rest of the state values $\left\{\hat{\xi}_{k+1 \mid k}^{N T}, \cdots, \hat{\xi}_{k+h-1 \mid k}^{N T}\right\}$ in order to replace the previous estimations $\left\{\hat{\xi}_{k+1 \mid k-1}^{N T}, \cdots, \hat{\xi}_{k+h-1 \mid k-1}^{N T}\right\}$. Additionally, the prediction stage generates a new state estimate $\hat{\xi}_{k+h \mid k}^{N T}$ to complete the set of predicted states. Excluding this new value and considering an accurate model and an acceptable level of noise, the difference between the previous and the current set of state values should be negligible.

\subsection{Motion Planning and Control Solution Design}

In the following subsections, each component of the motion planning and control layers of the AV structure will be defined in detail. The systematic combination of these components (EKF including $h$-step-ahead state prediction stage, pure pursuit path tracking algorithm, and dual-rate dynamic controller) is the most relevant contribution of this work. It enables maintaining the nominal control performance when path tracking and reduces the network bandwidth usage, despite the existence of time-varying network-induced delays and packet disorder and the appearance of process and measurement noise signals. Next, first of all, the AV model will be presented.

\subsubsection{Plant Modeling}

In this work, a non-linear kinematic model was utilized by the EKF in order to fulfill state estimation. Additionally, a dynamic relation between the control signal and the rotational velocity of the wheel was needed in order to design the dual-rate dynamic controller.

\subsubsection{Kinematic Model}

The kinematic model represents the AV velocity evolution in a fixed inertial frame. From the AV linear and rotational velocities in sampling period NT, i.e., $v_{k}^{N T}$ and $\omega_{k}^{N T}$, respectively, the $\mathrm{AV}$ position and orientation in the time period $N T$, i.e., $(x, y, \psi)_{k}^{N T}$, can be deduced as follows [44]: 


$$
\left(\begin{array}{l}
x \\
y \\
\psi
\end{array}\right)_{k}^{N T}=\left(\begin{array}{c}
x \\
y \\
\psi
\end{array}\right)_{k-1}^{N T}+\left(\begin{array}{c}
v_{k}^{N T} N T \cos \left(\psi_{k-1}^{N T}+\omega_{k}^{N T} N T\right) \\
v_{k}^{N T} N T \sin \left(\psi_{k-1}^{N T}+\omega_{k}^{N T} N T\right) \\
\omega_{k}^{N T} N T
\end{array}\right)
$$

for $k \in \mathbb{N}_{\geq 1}$, where $(x, y, \psi)_{0}^{N T}$ is the initial position and orientation and where the AV linear and rotational velocities come from:

$$
\left(\begin{array}{c}
v \\
w
\end{array}\right)_{k}^{N T}=\left(\begin{array}{cc}
1 / 2 & 1 / 2 \\
1 / 2 b & -1 / 2 b
\end{array}\right)\left(\begin{array}{c}
v_{r} \\
v_{l}
\end{array}\right)_{k}^{N T}
$$

being $b$ half of the distance between the wheels and $\left(v_{r}, v_{l}\right)_{k}^{N T}$ the linear velocities for each wheel, which are defined from the radius of the wheels, $r_{r}$ and $r_{l}$, and the consequent rotational velocities sensed in period $N T,\left(w_{r}, w_{l}\right)_{k}^{N T}$, as follows:

$$
\left(\begin{array}{l}
v_{r} \\
v_{l}
\end{array}\right)_{k}^{N T}=\left(\begin{array}{cc}
r_{r} & 0 \\
0 & r_{l}
\end{array}\right)\left(\begin{array}{c}
w_{r} \\
w_{l}
\end{array}\right)_{k}^{N T}
$$

\subsubsection{Dynamic Model}

The dynamic model represents the relation between the control signal and the rotational velocity for each wheel. Considering state-space representation, the model in sampling period $T$ takes this form:

$$
\left\{\begin{array}{l}
\left(x_{p}\right)_{k+1}^{T}=A \cdot\left(x_{p}\right)_{k}^{T}+B \cdot \hat{u}_{k}^{T}+\left(n_{1}\right)_{k}^{T} \\
\left(y_{p}\right)_{k}^{T}=C \cdot\left(x_{p}\right)_{k}^{T}+\left(n_{2}\right)_{k}^{T}
\end{array}\right.
$$

where, for the sake of simplicity, let us name:

- $\left(y_{p}\right)_{k}^{T}$ as the output, that is the rotational velocity either for the right motor $\left(w_{r}\right)_{k}^{T}$ or for the left motor $\left(w_{l}\right)_{k}^{T}$ and

- $\hat{u}_{k}^{T}$ as the input, that is the control signal, regardless of the motor, $\left(\hat{u}_{r}\right)_{k}^{T}$ or $\left(\hat{u}_{l}\right)_{k}^{T}$.

In addition, $\left(n_{1}\right)_{k}^{T}$ is the process noise, $\left(n_{2}\right)_{k}^{T}$ the measurement noise, $\left(x_{p}\right)_{k}^{T}$ the process state (regardless of the motor), and $A, B, C$ matrices with suitable dimensions. No unknown input is assumed. 
Using Z-transform in period $T$, the input-output plant model for the control signal and the rotational velocity is represented as a discrete-time transfer function:

$$
G_{p}^{T}(z)=Y_{p}^{T}(z) / \hat{U}^{T}(z)
$$

$z$ being the $T$-unit operator.

The plant model in (8.4) admits a lifted representation [45] such as:

$$
\left\{\begin{array}{l}
\left(x_{p}\right)_{k+N}^{T}=A^{N} \cdot\left(x_{p}\right)_{k}^{T}+\sum_{c=0}^{N-1} A^{N-1-c} \cdot B \cdot \hat{u}_{k+c}^{T}+\left(n_{1}\right)_{k}^{T} \\
\left(y_{p}\right)_{k}^{T}=C \cdot\left(x_{p}\right)_{k}^{T}+\left(n_{2}\right)_{k}^{T}
\end{array}\right.
$$

which can be equivalently seen as a dual-rate sampled-data system, where the output in period $N T,\left(y_{p}\right)_{k}^{N T}$, i.e., either $\left(w_{r}\right)_{k}^{N T}$ or $\left(w_{l}\right)_{k}^{N T}$, is obtained from a sequence of inputs in period $T$ included in the sensor period $N T$, i.e., $\hat{U}_{k}^{N T}=\left(\hat{u}_{k}^{T}, \hat{u}_{k+1}^{T}, \cdots, \hat{u}_{k+N-1}^{T}\right)^{\top}$, where $(\cdot)^{\top}$ means the transpose function, such as:

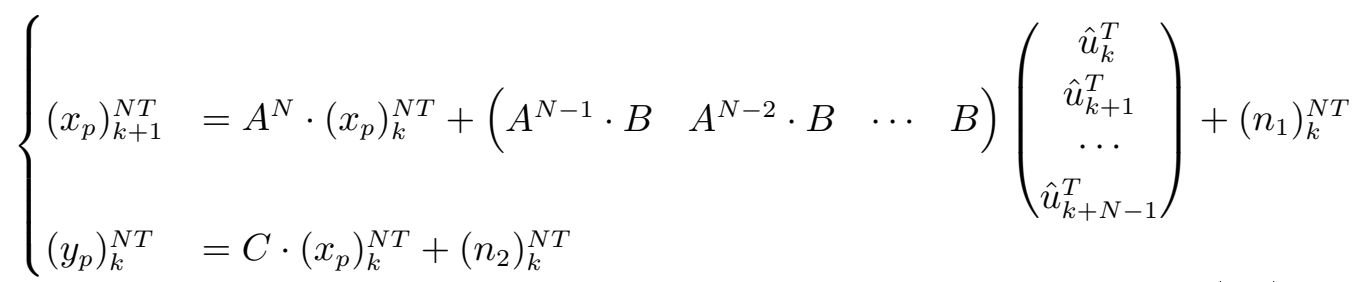

From (8.1) - (8.3) and (8.7), the global dynamic model for the AV was obtained. This model was used by the EKF to estimate the AV position and orientation.

\subsubsection{Extended Kalman Filter, Including an h-Step-ahead State Prediction Stage}

Since the AV model is non-linear, the use of Kalman filtering for state estimation may be considered through a linearization procedure, which is based on the use of a matrix of partial derivatives, that is a Jacobian matrix. At each time step, the Jacobian matrix is evaluated with current predicted states. This procedure results in an EKF (see, e.g., [30-32]). 
Let us denote the global non-linear dynamic model previously introduced by means of (8.1)-(8.3) and (8.7) as the next state-space representation:

$$
\begin{cases}\xi_{k}^{N T} & =f\left(\xi_{k-1}^{N T},\left(n_{1}\right)_{k-1}^{N T}, \hat{U}_{k-1}^{N T}\right) \\ z_{k}^{N T} & =h\left(\xi_{k}^{N T},\left(n_{2}\right)_{k}^{N T}\right)\end{cases}
$$

where we use the $\mathrm{AV}$ state $\xi_{k}^{N T}$ as $\left(\left(w_{r}, w_{l}, x, y, \psi\right)_{k}^{N T}\right)^{\top}$. As previously defined, $\hat{U}_{k}^{N T}$ is the control signal, and $\left(n_{1}\right)_{k}^{N T}$ and $\left(n_{2}\right)_{k}^{N T}$ are the process and measurement noises, respectively, which were both assumed to be zero mean multivariate Gaussian noises with covariance $Q_{k}^{N T}$ and $R_{k}^{N T}$, respectively.

Taking into account the notation introduced in subsection 8.2.1, where $\hat{\xi}_{j \mid i}^{N T}$ means the state estimated for the instant $j N T$ at the instant $i N T$, the prediction and correction steps of the EKF are defined as follows:

- Prediction of the next state $\hat{\xi}_{k \mid k-1}^{N T}$ and propagation of the covariance $P_{k \mid k-1}^{N T}$ :

$$
\begin{aligned}
& \hat{\xi}_{k \mid k-1}^{N T}=f\left(\hat{\xi}_{k-1 \mid k-1}^{N T},\left(n_{1}\right)_{k-1}^{N T}, \hat{U}_{k-1}^{N T}\right) \\
& P_{k \mid k-1}^{N T}=A_{k}^{N T} P_{k-1 \mid k-1}^{N T}\left(A_{k}^{N T}\right)^{\top}+L_{k}^{N T} Q_{k-1}^{N T}\left(L_{k}^{N T}\right)^{\top}
\end{aligned}
$$

for $k \in \mathbb{N}_{\geq 1}$, where $\hat{\xi}_{0}^{N T}=E\left[\xi_{0}^{N T}\right]$ and $P_{0}^{N T}=E\left[\left(\xi_{0}^{N T}-E\left[\xi_{0}^{N T}\right]\right)\left(\xi_{0}^{N T}-\right.\right.$ $\left.\left.E\left[\xi_{0}^{N T}\right]\right)^{\top}\right], E[\cdot]$ being the expectation, and where $A_{k}^{N T}$ and $L_{k}^{N T}$ are Jacobian matrices computed in order to linearize respectively the process model about the current state and about the process noise:

$$
\begin{aligned}
& A_{k}^{N T}=\left.\frac{\partial f}{\partial \xi}\right|_{\hat{\xi}_{k-1 \mid k-1}^{N T},\left(n_{1}\right)_{k-1}^{N T}, \hat{U}_{k-1}^{N T}} \\
& L_{k}^{N T}=\left.\frac{\partial f}{\partial n_{1}}\right|_{\hat{\xi}_{k-1 \mid k-1}^{N T},\left(n_{1}\right)_{k-1}^{N T}, \hat{U}_{k-1}^{N T}}
\end{aligned}
$$

- Prediction of the future output $\hat{z}_{k}^{N T}$ and computation of the Kalman filter gain $K_{k}^{N T}$ : 


$$
\begin{aligned}
\hat{z}_{k}^{N T} & =h\left(\hat{\xi}_{k \mid k-1}^{N T},\left(n_{2}\right)_{k}^{N T}\right) \\
K_{k}^{N T} & =P_{k \mid k-1}^{N T}\left(H_{k}^{N T}\right)^{\top}\left(H_{k}^{N T} P_{k \mid k-1}^{N T}\left(H_{k}^{N T}\right)^{\top}+M_{k}^{N T} R_{k}^{N T}\left(M_{k}^{N T}\right)^{\top}\right)^{-1}
\end{aligned}
$$

where $H_{k}^{N T}$ and $M_{k}^{N T}$ are Jacobian matrices calculated in order to linearize respectively the output model about the predicted next state and about the measurement noise:

$$
\begin{aligned}
H_{k}^{N T} & =\left.\frac{\partial h}{\partial \xi}\right|_{\hat{\xi}_{k \mid k-1}^{N T},\left(n_{2}\right)_{k}^{N T}} \\
M_{k}^{N T} & =\left.\frac{\partial h}{\partial n_{2}}\right|_{\hat{\xi}_{k \mid k-1}^{N T},\left(n_{2}\right)_{k}^{N T}}
\end{aligned}
$$

- Correction of the state $\hat{\xi}_{k \mid k}^{N T}$ and correction of the covariance $P_{k \mid k}^{N T}$ :

$$
\begin{aligned}
\hat{\xi}_{k \mid k}^{N T} & =\hat{\xi}_{k \mid k-1}^{N T}+K_{k}^{N T}\left(z_{k}^{N T}-\hat{z}_{k}^{N T}\right) \\
P_{k \mid k}^{N T} & =K_{k}^{N T} R_{k}^{N T}\left(K_{k}^{N T}\right)^{\top}+\left(I-K_{k}^{N T} H_{k}^{N T}\right) P_{k \mid k-1}^{N T}\left(I-K_{k}^{N T} H_{k}^{N T}\right)^{\top}
\end{aligned}
$$

Additionally, the EKF integrated an $h$-step-ahead state prediction stage, whose working mode is described in the next items:

1. The state corrected in (8.13) was used, together with the kinematic reference $\left(x^{r e f}, y^{r e f}, \psi^{r e f}\right)_{k}^{N T}$, by the path tracking algorithm in order to calculate the dynamic reference to be followed by each wheel, $\left(\hat{w}_{r}^{\text {com }}, \hat{w}_{l}^{\text {com }}\right)_{k}^{N T}$. More details about this calculation will be given in subsection 8.3.3.

2. From these dynamic references and the corrected rotational velocities $\left(\hat{w}_{r}, \hat{w}_{l}\right)_{k}^{N T}$, the dynamic controller was able to compute the control signal $\hat{U}_{k}^{N T}$ for the current sensing period. More details about this computation will be provided in subsection 8.3.4.

3. Following an open-loop dynamics-based prediction, the non-linear model of the AV in (8.8) was iterated from the estimated state and the control signal in order to obtain the next state and output estimations, $\hat{\xi}_{k+1}^{N T}$ and $\hat{z}_{k+1}^{N T}$, respectively: 


$$
\left\{\begin{array}{l}
\hat{\xi}_{k+1}^{N T}=f\left(\hat{\xi}_{k}^{N T},\left(n_{1}\right)_{k}^{N T}, \hat{U}_{k}^{N T}\right) \\
\hat{z}_{k+1}^{N T}=h\left(\hat{\xi}_{k+1}^{N T},\left(n_{2}\right)_{k+1}^{N T}\right)
\end{array}\right.
$$

4. Finally, Steps $1-3$ were repeated $h-1$ times to compute the rest of the values of the set of estimates $\left\{\hat{\xi}_{k+2}^{N T}, \cdots, \hat{\xi}_{k+h}^{N T}\right\}$.

\subsubsection{Pure Pursuit Path Tracking Algorithm}

From the desired kinematic reference $\left(x^{r e f}, y^{r e f}, \psi^{r e f}\right)_{k}^{N T}$ and the state corrected by the EKF in period $N T, \hat{\xi}_{k}^{N T}$, the AV was able to infer its current position using a path tracking algorithm. As commented, in this work, pure pursuit was chosen (see, e.g., [25-27]), which generated the dynamic reference based on the rotational velocity for both wheels, i.e., $\left(\hat{w}_{r}^{\text {com }}, \hat{w}_{l}^{c o m}\right)_{k}^{N T} \equiv\left(\hat{y}_{p}^{c o m}\right)_{k}^{N T}$, in order to reach properly the next point of the desired trajectory.

The reference generator was in charge of providing the pure pursuit algorithm with a sequence of $h$-step-ahead kinematic references, $\left\{\left(x^{r e f}, y^{r e f}, \psi^{r e f}\right)_{k}^{N T}\right.$, $\left.\left(x^{r e f}, y^{r e f}, \psi^{r e f}\right)_{k+1}^{N T}, \cdots,\left(x^{r e f}, y^{r e f}, \psi^{r e f}\right)_{k+h}^{N T}\right\}$. From these references and the $h$-step-ahead estimations, the AV path tracking was able to establish the set of $h$ future dynamic references, $\left\{\left(\hat{y}_{p}^{\text {com }}\right)_{k}^{N T},\left(\hat{y}_{p}^{\text {com }}\right)_{k+1}^{N T}, \cdots,\left(\hat{y}_{p}^{\text {com }}\right)_{k+h}^{N T}\right\}$.

The pure pursuit algorithm was based on the computation of the curvature $\bar{\gamma}$ that a vehicle must adopt from its current position $(x, y)$ to a target position $(x+\Delta x, y+\Delta y)$. A circle of radius $r$ that joins both points can be considered, the center of the circle being in $x+\Delta x+d$. In addition, the distance to the target point is $L$.

Therefore, it is possible to express:

$$
\begin{aligned}
r & =\Delta x+d \\
L^{2} & =(\Delta x)^{2}+(\Delta y)^{2}
\end{aligned}
$$

being the curvature:

$$
\bar{\gamma}=\frac{(\Delta x)^{2}+(\Delta y)^{2}}{2 \Delta x}
$$

and the so-called pure pursuit control law: 


$$
\bar{k}=\frac{1}{\bar{\gamma}}=\frac{2 \Delta x}{L^{2}}
$$

Therefore, the control law $\bar{k}$ is proportional to the lateral shift and inversely proportional to the square of $L$.

The pure pursuit path tracking algorithm requires determining the target, reference position $\left(x_{P P}^{r e f}, y_{P P}^{r e f}\right)_{k}^{N T}$ located at a minimum distance from the current position $(\hat{x}, \hat{y})_{k}^{N T}$, i.e., the so-called Look Ahead Distance (LAD), not considering the nearest points in the prescribed trajectory. This procedure avoids a severe correction, and hence, it leads to a soft movement. Assuming $\left(x_{P P}^{r e f}, y_{P P}^{r e f}\right)_{k}^{N T}$ and the current position and orientation $(\hat{x}, \hat{y}, \hat{\psi})_{k}^{N T}$, the pure pursuit control law $\bar{k}$ can be expressed as follows:

$$
\bar{k}=2 \frac{\left(y_{P P}^{r e f}-\hat{y}\right) \cos (\hat{\psi})-\left(x_{P P}^{r e f}-\hat{x}\right) \sin (\hat{\psi})}{\left(x_{P P}^{r e f}-\hat{x}\right)^{2}+\left(y_{P P}^{r e f}-\hat{y}\right)^{2}}
$$

For a desired linear velocity $\left(v^{r e f}\right)_{k}^{N T}$, the rotational velocity reference $\left(\omega^{r e f}\right)_{k}^{N T}$ can be calculated as:

$$
\left(\omega^{r e f}\right)_{k}^{N T}=\left(v^{r e f}\right)_{k}^{N T} \bar{k}
$$

As a summary, these are the steps to be followed when the main loop of the pure pursuit algorithm is implemented:

1. Generation of the future reference for the robot, $\left(x_{P P}^{r e f}, y_{P P}^{r e f}\right)_{k}^{N T}$ : From the desired kinematic reference and the Look Ahead Distance (LAD), the nearest point to the future path tracking that was located far away from the LAD was calculated.

2. Control law computation: From $\left(x_{P P}^{r e f}, y_{P P}^{r e f}\right)_{k}^{N T}$ and the position and orientation estimate provided by the EKF, $(\hat{x}, \hat{y}, \hat{\psi})_{k}^{N T}$, the control law $\bar{k}$ was computed by using (8.18), and then, $\left(\omega^{r e f}\right)_{k}^{N T}$ was calculated for each wheel by using (8.19), requiring a desired $\left(v^{r e f}\right)_{k}^{N T}$. Finally, from these data, $\left(\hat{w}_{r}^{\text {com }}, \hat{w}_{l}^{\text {com }}\right)_{k}^{N T} \equiv\left(\hat{y}_{p}^{\text {com }}\right)_{k}^{N T}$ could be calculated:

$$
\left(\begin{array}{c}
\hat{w}_{r}^{\text {com }} \\
\hat{w}_{l}^{\text {com }}
\end{array}\right)_{k}^{N T}=\left(\begin{array}{cc}
1 / r_{r} & b / r_{r} \\
1 / r_{l} & -b / r_{l}
\end{array}\right)\left(\begin{array}{c}
v^{r e f} \\
\omega^{r e f}
\end{array}\right)_{k}^{N T}
$$


where $b$ was previously defined as half of the distance between the wheels and $r_{r}$ and $r_{l}$ as the radius of each wheel.

\subsubsection{Dual-Rate Controller}

In this work, in order to reach the desired control performance, a dual-rate controller was used. From NT-period signals such as the dynamic reference $\left(\hat{y}_{p}^{\text {com }}\right)_{k}^{N T}$ generated by the pure pursuit path tracking algorithm and the estimated, corrected rotational velocities $\left(\hat{y}_{p}\right)_{k}^{N T}$ obtained by the EKF, the dynamic dual-rate controller computed $N$ control actions in period $T$ for each wheel inside the current sensor period NT. This control signal was previously defined as $\hat{U}_{k}^{N T}=\left(\hat{u}_{k}^{T}, \hat{u}_{k+1}^{T}, \ldots, \hat{u}_{k+N-1}^{T}\right)^{\top}$. Following this operation mode for the next $h$ dynamic references and rotational velocities, $\left\{\left(\hat{y}_{p}^{c o m}\right)_{k+1}^{N T}, \ldots,\left(\hat{y}_{p}^{c o m}\right)_{k+h}^{N T}\right\}$ and $\left\{\left(\hat{y}_{p}\right)_{k+1}^{N T}, \ldots,\left(\hat{y}_{p}\right)_{k+h}^{N T}\right\}$, respectively, the set of future control actions $\left\{\hat{u}_{k+N}^{T}, \ldots, \hat{u}_{k+h N-1}^{T}\right\}$ can be obtained.

Different alternatives can be followed to design a dual-rate controller (see, e.g., in $[37,38])$. In this case, the model-based dual-rate controller design described in [37] was chosen, where the cascade structure of the controller included:

- A slow-rate sub-controller: $G_{1}^{N T}\left(z^{N}\right)=u_{1, k}^{N T} / e_{k}^{N T}$.

- A digital hold: $H^{N T, T}(z)=u_{1, k}^{T} /\left[u_{1, k}^{N T}\right]^{T}$.

- A fast-rate sub-controller: $G_{2}^{T}(z)=\hat{U}_{k}^{T} / u_{1, k}^{T}$.

The input of $G_{1}^{N T}\left(z^{N}\right)$ was the error signal $e_{k}^{N T}=\left(\hat{y}_{p}^{c o m}\right)_{k}^{N T}-\left(\hat{y}_{p}\right)_{k}^{N T}$. Note that the output of $G_{1}^{N T}\left(z^{N}\right)$ (i.e., $\left.u_{1, k}^{N T}\right)$ was expanded, $\left[u_{1, k}^{N T}\right]^{T}$, before being injected into the digital hold $H^{N T, T}(z)$. The expand operation implied filling the slow-rate signal with zeros at the fast-rate instants (more details can be found in [37]). Then, the digital hold obtained its output $u_{1, k}^{T}$ by means of:

$$
H^{N T, T}(z)=\frac{1-z^{-N}}{1-z^{-1}}
$$

which in conclusion resulted in the sub-controller output $u_{1, k}^{N T}$ being repeated $N$ times. From the consideration of $M(s)$ as the desired closed-loop control performance of the original continuous-time system design, the sub-controllers $G_{1}^{N T}\left(z^{N}\right)$ and $G_{2}^{T}(z)$ were designed as follows: 


$$
\begin{aligned}
G_{1}^{N T}\left(z^{N}\right) & =\frac{1}{1-M^{N T}\left(z^{N}\right)} \\
G_{2}^{T}(z) & =\frac{M^{T}(z)}{G_{p}^{T}(z)}
\end{aligned}
$$

where $G_{p}^{T}(z)$ was presented in (8.5) and came from the discretization of the continuous-time plant model in period $T$ under $\mathrm{ZOH}$ conditions and $M^{T}(z)$ and $M^{N T}\left(z^{N}\right)$ are the discretization of $M(s)$ in periods $T$ and $N T$, respectively, using $\mathrm{ZOH}$ techniques, as well.

\subsection{Cost Indexes for Control Performance}

In this section, three cost indexes closely req:paper7:6o control performance will be presented. By means of these indexes, the remote control solution may be compared with a conventional, single-rate control strategy. Similarly to [46], the indexes used were:

- $J_{1}$, which is based on the $\ell_{2}$-norm, and its goal is to provide a measure about how accurately the path was followed:

$$
J_{1}=\sum_{k=1}^{l} \min _{1 \leq k^{\prime} \leq l} \sqrt{\left\{x_{k}^{N T}-\bar{x}_{k^{\prime}}^{N T}\right\}^{2}+\left\{y_{k}^{N T}-\bar{y}_{k^{\prime}}^{N T}\right\}^{2}}
$$

where $l$ is the number of iterations required by the AV to reach the final point of the path, $(x, y)_{k}^{N T}$ is the current AV position, and $(\bar{x}, \bar{y})_{k^{\prime}}^{N T}$ is the nearest kinematic position reference to the current AV position.

- $J_{2}$, which is based on the $\ell_{\infty}$-norm and is defined to know the maximum difference between the desired path and the current AV position:

$$
J_{2}=\max _{1 \leq k \leq l}\left\{\min _{1 \leq k^{\prime} \leq l} \sqrt{\left\{x_{k}^{N T}-\bar{x}_{k^{\prime}}^{N T}\right\}^{2}+\left\{y_{k}^{N T}-\bar{y}_{k^{\prime}}^{N T}\right\}^{2}}\right\}
$$

- $J_{3}$, which measures the total amount of time (in seconds) elapsed to arrive at the final destination:

$$
J_{3}=l N T
$$




\subsection{Application}

In this section, control properties reached by the remote control solution will be compared to those achieved by several single-rate, conventional control approaches defined at different rates. The section is split into two parts. Firstly, the application data will be presented, that is the different parameters for the AV model, network, and control systems. Secondly, the cost indexes introduced in section 8.4 will be evaluated for the different control approaches by means of a Truetime application [42]. The comparison results will show that the proposed remote control solution was able to keep the desired control properties for the AV path tracking, despite sensing at a slow rate, the existence of process and measurement noises, and considering time-varying network-induced delays and packet disorder. In addition, the control proposal achieved a significant reduction of the network bandwidth.

\subsubsection{Data}

These were the data used in the application:

- The AV was a Lego robot with two wheel motors (shown in Figure 8.4). Considering a similar model for both motors, the dynamic model for the relation between rotational velocity of the wheel and control signal is:

$$
G_{p}(s)=\frac{Y_{p}(s)}{\hat{U}(s)}=\frac{0.1276}{0.1235 s+1}
$$

where the output is in rad/s and the input in V. From (8.26), the consequent state space realization (8.4) can be obtained in order to be used in (8.7) to calculate the rotational velocities and then the rest of the elements of the state estimated by the EKF and the $h$-step-ahead state prediction stage.

- As typical in Ethernet environments [47], a generalized exponential distribution for the time-varying network-induced delays was assumed, in this case being the maximum time delay $\tau_{\max }=0.17 \mathrm{~s}$. In order to avoid packet disorder, the sampling time $N T$ was chosen to be $N T=0.2 \mathrm{~s}$.

- From (8.26) and following classical procedures [48, 49], a PI controller can be designed in order to achieve certain specifications. Taking into consideration this typical PI configuration: 


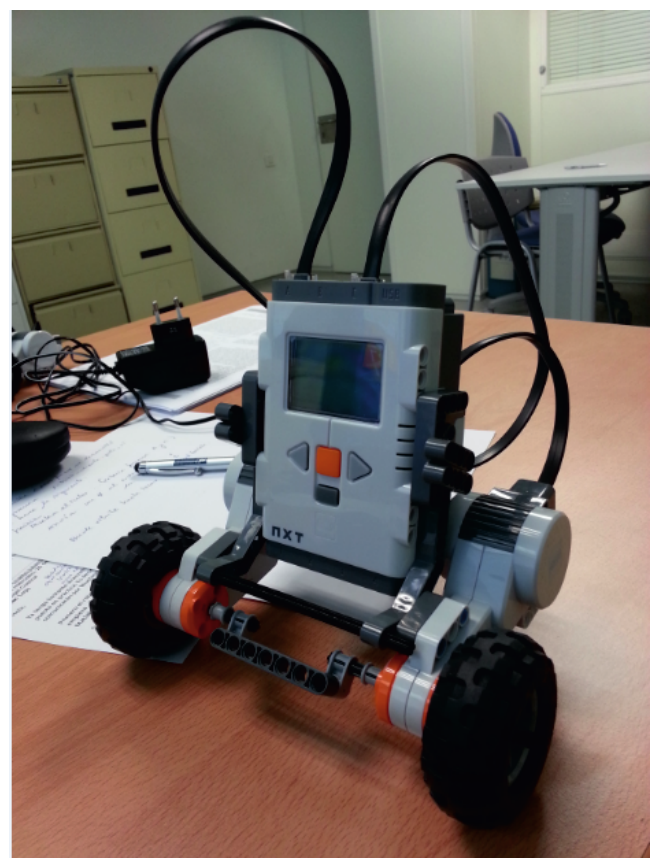

Figure 8.4: Lego robot

$$
u(t)=K_{p}\left[e(t)+\frac{1}{T_{i}} \int_{0}^{t} e(\tau) d \tau\right]
$$

the controller's gains will be tuned such as $K_{p}=6$ and $T_{i}=0.12$. In order to obtain a satisfactory control performance, the actuation period was selected to be $T=0.1 \mathrm{~s}$, and hence, the multiplicity was $N=2$. From these values and the discretization of (8.26) in period $T$, a dual-rate PI controller can be designed by means of (8.21) and (8.22), bringing about:

$$
\begin{aligned}
G_{1}^{N T}\left(z^{N}\right) & =\frac{z^{2}-0.4734 z+0.05731}{z^{2}-1.191 z+0.1914} \\
G_{2}^{T}(z) & =\frac{6.576 z^{2}-5.78 z+1.27}{z^{2}-0.9578 z+0.2394}
\end{aligned}
$$


- For the comparison between dual-rate and single-rate control approaches, the continuous-time PI controller in (8.27) was discretized in the different periods $T$ and $N T$. The single-rate controllers are:

$$
\begin{gathered}
G_{r}^{T}(z)=\frac{6 z-1}{z-1} \\
G_{r}^{N T}\left(z^{N}\right)=\frac{6 z+4}{z-1}
\end{gathered}
$$

- The control solution was evaluated under different levels of noise in order to study the effect of the process and sensor noises on the performance. Let us consider a lower level of noise, where both noise signals are multiplied by a lower factor $F=0.1$, and a higher level of noise, where $F=$ 0.45 . By simulation, it was checked that, from $F=0.45$, the robustness of the control proposal may be compromised.

- Finally, the reference to be followed included a sequence of four right angles.

\subsubsection{Results}

In the next figures, the path reference is depicted in black, and the trajectory followed by the AV is shown in blue.

Figure 8.5 shows the results for the single-rate control in period $T=0.1 \mathrm{~s}$ in (8.30). In this simulation, neither delays and noise signals, nor additional control techniques (such as EKF, $h$-step-ahead prediction stage, and packet-based control) were considered. Let us define the behavior depicted in Figure 8.5 as the nominal behavior.

In Figure 8.6, the sampling period was increased twice, and then, the single-rate control in period $N T=0.2 \mathrm{~s}$ in (8.31) was considered. The rest of simulation conditions were the same as in the previous case. Now, compared to the singlerate version in period $T=0.1 \mathrm{~s}$, the control performance clearly worsened when the AV tried to track the path.

If the sensing period was kept at $N T=0.2 \mathrm{~s}$, but the actuation period was $N=2$-times faster (i.e., in period $T=0.1 \mathrm{~s}$ ), the dual-rate control scheme in (8.28) and (8.29) could be taken into account, which was able to maintain a satisfactory control performance, being very similar to the nominal one (see 


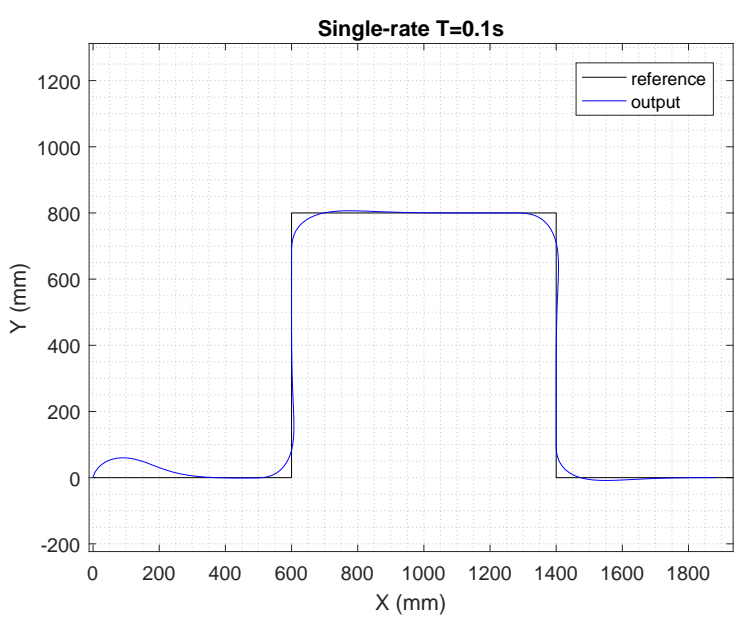

Figure 8.5: Results for the single-rate control at $T=0.1 \mathrm{~s}$

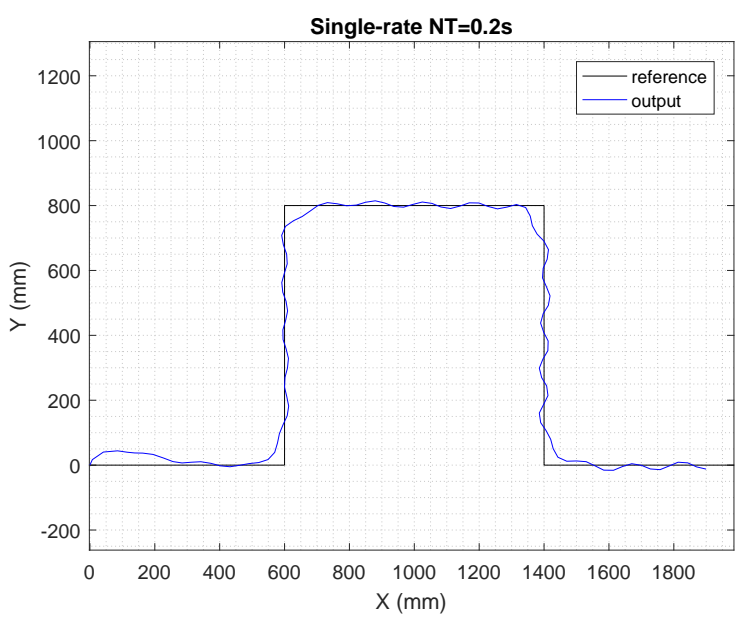

Figure 8.6: Results for the single-rate control at $N T=0.2 \mathrm{~s}$

Figure 8.7). However, if time-varying network-induced delays were included in the dual-rate control framework, the AV's behavior clearly worsened, as depicted in Figure 8.8.

Finally, despite existing time-varying delays and noise, if the additional control techniques proposed in this work such as EKF, $h$-step-ahead prediction stage, 


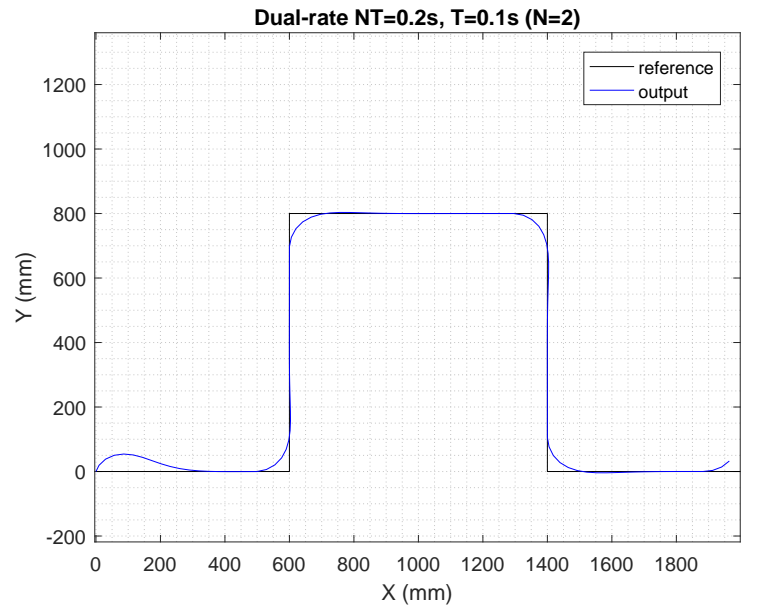

Figure 8.7: Results for the dual-rate control at $N T=0.2 \mathrm{~s}$ and $T=0.1 \mathrm{~s}$

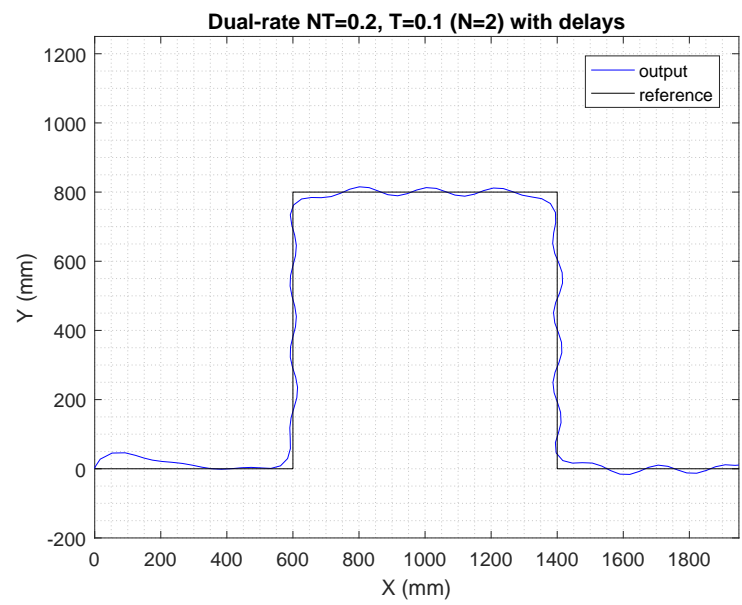

Figure 8.8: Results for the dual-rate control with delays

and packet-based control were integrated in the dual-rate control scheme, control performance was clearly improved, keeping the nominal behavior (see in Figure 8.9, where the case for the lower level of noise was considered; Figure 8.10 shows the control signal for this case). In addition, state estimates can be received at the remote server for further upper level tasks. In Table 8.1 , an example considering $h=1$ is given, where the set of estimations 
was $\left[\left(\hat{w}_{r}, \hat{w}_{l}, \hat{x}, \hat{y}, \hat{\psi}\right)_{k},\left(\hat{w}_{r}, \hat{w}_{l}, \hat{x}, \hat{y}, \hat{\psi}\right)_{k+1}\right]$. Note that the first value of the set of estimations was the corrected system state, and the second value was the estimation of the next state. A slight correction of the state can be observed iteration by iteration.

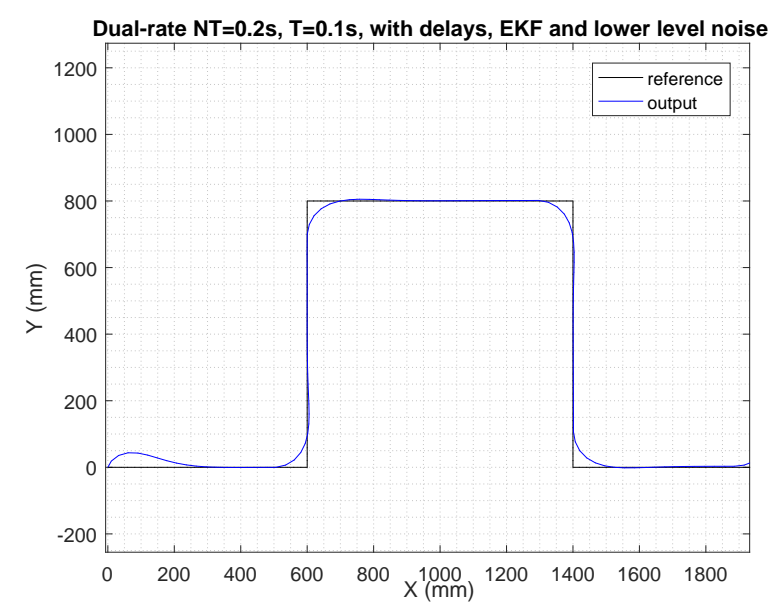

Figure 8.9: Results for the control proposal (EKF, prediction stage, and packet-based control) with delays and a lower level of noise
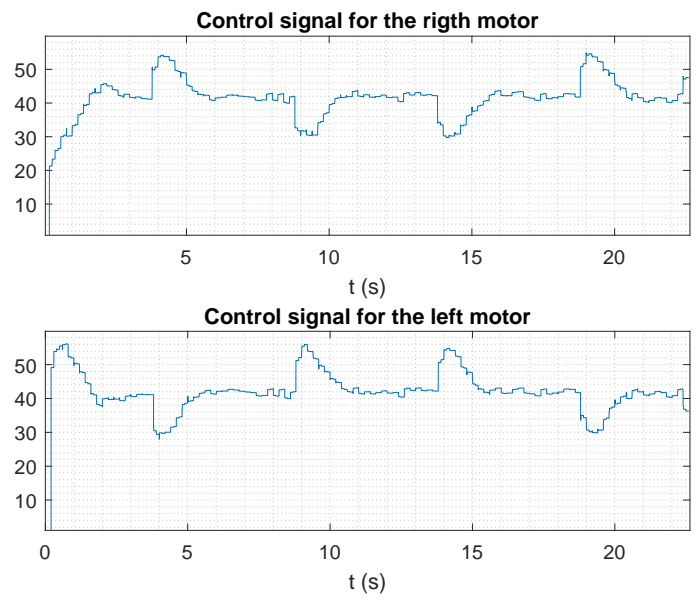

Figure 8.10: Control signal for the control proposal (EKF, prediction stage, and packetbased control) with delays and a lower level of noise 


\begin{tabular}{|l|ll|}
\hline$k$ & $\left(\hat{w}_{r}, \hat{w}_{l}, \hat{x}, \hat{y}, \hat{\psi}\right)_{k}$ & $\left(\hat{w}_{r}, \hat{w}_{l}, \hat{x}, \hat{y}, \hat{\psi}\right)_{k+1}$ \\
\hline 0 & $\left((0.5349,0.5349,0.4621,2.9599) \cdot 10^{-5},-30\right)$ & $(2.3264,5.3668,9.5421,19.3122,-30.3040)$ \\
1 & $(2.3264,5.3668,9.5421,19.3122,-30.3040)$ & $(3.1705,6.7276,29.7038,38.3284,-30.6598)$ \\
2 & $(3.1700,6.7265,29.6994,38.3267,-30.6597)$ & $(3.5737,6.9281,56.5393,50.3383,-30.9951)$ \\
3 & $(3.5739,6.9284,56.5408,50.3384,-30.9951)$ & $(3.8830,6.7774,86.1327,54.2475,-31.2846)$ \\
\hline
\end{tabular}

Table 8.1: Example for $h=1$

The two last figures are presented (Figure 8.11 and Figure 8.12), where the same previous control solution was simulated, but now considering the case for the higher level of noise. As depicted, the control signal was affected by this significant magnitude of noise, which negatively affected path tracking control. Since this case represents an extreme level of noise, it will not be considered in the next study.

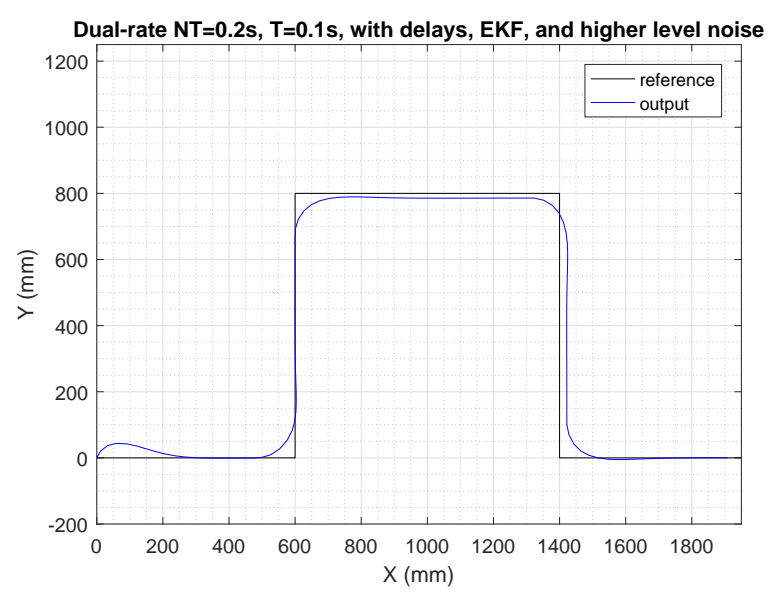

Figure 8.11: Results for the control proposal (EKF, prediction stage, and packet-based control) with delays and a higher level of noise

To analyze the previous conclusions in more detail, the cost indexes presented in section 8.4 were calculated for every scenario. Table 8.2 shows these results, where each scenario is represented by the following letters:

- a: single-rate control scenario in period $T$.

- b: single-rate control scenario in period $N T$.

- c: dual-rate control scenario. 

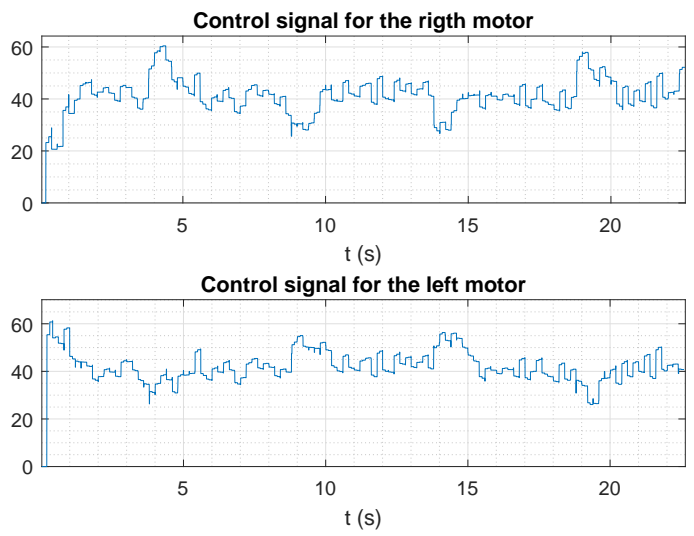

Figure 8.12: Control signal for the control proposal (EKF, prediction stage, and packetbased control) with delays and a higher level of noise

\begin{tabular}{|l|lllll|}
\hline index & $\mathrm{a}$ & $\mathrm{b}$ & $\mathrm{c}$ & $\mathrm{d}$ & $\mathrm{e}$ \\
\hline$J_{1}$ & 1043.4 & 1671.8 & 1029.9 & 1684.4 & 1030.0 \\
$J_{2}$ & 38.76 & 44.55 & 38.33 & 44.33 & 38.97 \\
$J_{3}$ & $22.0 \mathrm{~s}$ & $22.4 \mathrm{~s}$ & $22.0 \mathrm{~s}$ & $21.6 \mathrm{~s}$ & $21.6 \mathrm{~s}$ \\
\hline
\end{tabular}

Table 8.2: Cost indexes for each scenario

- d: dual-rate control scenario with delays.

- e: dual-rate control scenario, adding EKF, $h$-step-ahead prediction stage, and packet-based control. Delays and a lower level of noise were considered.

As previously mentioned, the desired, nominal performance was presented by Scenario a, and hence, its $J_{1}, J_{2}, J_{3}$ showed the reference values to carry out the comparison. As expected, Scenarios b and d showed the worst $J_{1}(62 \%$ worse than the nominal value), since the desired trajectory was inaccurately followed by the AV. Scenarios c and e showed a similar $J_{1}$ as Scenario a (even $1 \%$ better). Regarding index $J_{2}$, it was worsened around $16 \%$ by Scenarios $\mathrm{b}$ and d compared to the nominal performance, which was practically reached by Scenarios c and e. Index $J_{3}$ presented similar values for every case, differing only by $\pm 1 \%$ with respect to the nominal value. Finally, it is worthy to note that Scenarios b, c, d, and e were able to reduce the use of the network by $50 \%$, due to sampling it twice slower. This fact implies considerable bandwidth saving. 
In summary, the proposed remote control solution was able to keep the desired control properties for the AV path tracking, despite slow sensing and the existence of noise and wireless communication problems such as time-varying delays. In addition, the control proposal significantly reduced network resource usage $(50 \%)$, avoided packet disorder, and provided the remote server with future system information.

\subsection{Conclusions}

A novel remote control solution for AV path tracking was presented, which integrated dual-rate control, packed-based control, extended Kalman filtering, and prediction-based control techniques. A desired, nominal control performance was defined by a single-rate control at a fast rate in an ideal framework, where no wireless communication problems (such as time-varying delays and packet disorder) and no noise effect were considered. The proposal was able to reach the nominal behavior, despite communication problems and noise existing and despite sensing at a slow rate due to technical constraints introduced by the zenithal camera. In addition, the control approach enabled lessening the amount of transmission through the network, which can be decreased $N$ times in comparison with the ideal framework, resulting in significant bandwidth savings.

\section{Author Contributions}

Conceptualization, A.C., W.Z., and C.T.; methodology, A.C., W.Z., J.S., and M.T.; software, A.C., C.T., J.S., and J.A.; formal analysis, A.C. and J.A.; writing, original draft preparation, A.C.; writing, review and editing, A.C., W.Z., J.S., J.A., C.T., and M.T.; supervision, J.S. and M.T.; funding acquisition, A.C. and J.S.

\section{Funding}

This research work has been developed as a result of a mobility stay funded by Spanish Ministry of Education under "Programa Estatal de Promoción del Talento y su Empleabilidad en $\mathrm{I}+\mathrm{D}+\mathrm{i}$, Subprograma Estatal de Movilidad, del Plan Estatal de Investigación Científica y Técnica y de Innovación 2013-2016". In addition, the research was funded in part by Grant RTI2018096590-B-I00 from the Spanish government and by the European Commis- 
sion as part of Project H2020-SEC-2016-2017, Topic: SEC-20-BES-2016, ID: 740736, "C2 Advanced Multi-domain Environment and Live Observation Technologies" (CAMELOT). Part WP5 supported by Tekever ASDS, Thales Research and Technology, Viasat Antenna Systems, Universitat Politècnica de València, Fundação da Faculdade de Ciências da Universidade de Lisboa, Ministério da Defensa Nacional, Marinha Portuguesa, and Ministério da Administração Interna Guarda Nacional Republicana.

\section{Conflicts of interest}

The authors declare no conflict of interest.

\section{Abbreviations}

The following abbreviations are used in this manuscript:

AV Autonomous Vehicle

IMU Inertial Measurement Unit

EKF Extended Kalman Filter

ZOH Zero Order Hold

LAD Look Ahead Distance 


\section{References}

[1] Rachana Ashok Gupta and Mo-Yuen Chow. "Networked control system: Overview and research trends". In: 57.7 (2010), pp. 2527-2535 (cit. on p. 238).

[2] L. Zhang, H. Gao, and O. Kaynak. "Network-Induced Constraints in Networked Control Systems - A Survey". In: IEEE Transactions on Industrial Informatics 9.1 (Feb. 2013), pp. 403-416. ISSN: 1551-3203. DOI: 10.1109/TII.2012.2219540 (cit. on p. 238).

[3] Xian-Ming Zhang, Qing-Long Han, and Xinghuo Yu. "Survey on Recent Advances in Networked Control Systems". In: IEEE Transactions on Industrial Informatics 12.5 (Oct. 2016), pp. 1740-1752. ISSN: 1551-3203. DOI: 10.1109/TII.2015.2506545 (cit. on p. 238).

[4] Dan Zhang et al. "Analysis and synthesis of networked control systems: A survey of recent advances and challenges". In: ISA Transactions 66 (2017), pp. 376-392. ISSN: 0019-0578. DOI: https://doi.org/10.1016/ j.isatra.2016.09.026 (cit. on p. 238).

[5] Yun-Bo Zhao et al. "Exploring the Different Delay Effects in Different Channels in Networked Control Systems". In: Packet-Based Control for Networked Control Systems: A Co-Design Approach. Singapore: Springer Singapore, 2018, pp. 99-113. ISBN: 978-981-10-6250-6. DOI: $10.1007 /$ 978-981-10-6250-6_8 (cit. on p. 238).

[6] Wenlong Zhang et al. "A double disturbance observer design for compensation of unknown time delay in a wireless motion control system". In: 26.2 (2018), pp. 675-683 (cit. on p. 238).

[7] Á. Cuenca et al. "Periodic Event-Triggered Sampling and Dual-Rate Control for a Wireless Networked Control System With Applications to UAVs". In: IEEE Transactions on Industrial Electronics 66.4 (Apr. 2019), pp. 3157-3166. ISSN: 0278-0046. DOI: 10.1109/TIE. 2018.2850018 (cit. on p. 238). 
[8] Xian-Ming Zhang and Qing-Long Han. "Event-triggered dynamic output feedback control for networked control systems". In: IET Control Theory Appl. 8.4 (2014), pp. 226-234 (cit. on p. 238).

[9] Arman Sargolzaei, Kang K Yen, and Mohamed N Abdelghani. "Preventing time-delay switch attack on load frequency control in distributed power systems". In: IEEE Trans. Smart Grid 7.2 (2015), pp. 1176-1185 (cit. on p. 238).

[10] Arman Sargolzaei et al. "Resilient design of networked control systems under time delay switch attacks, application in smart grid". In: IEEE Access 5 (2017), pp. 15901-15912 (cit. on p. 238).

[11] Wenlong Zhang, Joonbum Bae, and Masayoshi Tomizuka. "Modified preview control for a wireless tracking control system with packet loss". In: IEEE/ASME Trans. Mechatronics 20.1 (2015), pp. 299-307 (cit. on p. 238).

[12] Yu-Long Wang and Qing-Long Han. "Network-based modelling and dynamic output feedback control for unmanned marine vehicles in network environments". In: Automatica 91 (2018), pp. 43-53. ISSN: 0005-1098. DOI: https://doi.org/10.1016/j.automatica.2018.01.026 (cit. on p. 238).

[13] J. Alcaina et al. "Delay-independent dual-rate PID controller for a packetbased networked control system". In: Information Sciences 484 (2019), pp. 27-43. ISSN: 0020-0255. DOI: https ://doi.org/10.1016/j.ins . 2019.01.059 (cit. on p. 238).

[14] A. Liu et al. "Networked filtering with Markov transmission delays and packet disordering". In: IET Control Theory Applications 12.5 (2018), pp. 687-693. ISSN: 1751-8644. DOI: 10.1049/iet-cta. 2017.0755 (cit. on p. 238).

[15] Andong Liu et al. "New results on stabilization of networked control systems with packet disordering". In: Automatica 52 (2015), pp. 255259. ISSN: 0005-1098. DOI: https://doi.org/10.1016/j.automatica. 2014.12.006 (cit. on p. 238).

[16] Bosen Lian, Qingling Zhang, and Jinna Li. "Sliding mode control and sampling rate strategy for Networked control systems with packet disordering via Markov chain prediction". In: ISA Trans. 83 (2018), pp. 1-12 (cit. on p. 238).

[17] Ling-Li Cheng et al. "An Optimal Tracking Performance of MIMO NCS with Quantization and Bandwidth Constraints". In: Asian J. Control 21.4 (2019), pp. 1-12 (cit. on p. 238). 
[18] Ricardo Emerson Julio and Guilherme Sousa Bastos. "A ROS Package for Dynamic Bandwidth Management in Multi-robot Systems". In: Robot Operating System (ROS): The Complete Reference (Volume 2). Ed. by Anis Koubaa. Cham: Springer International Publishing, 2017, pp. 309341. ISBN: 978-3-319-54927-9. DOI: 10 . 1007/978-3-319-54927-9_10 (cit. on p. 238).

[19] Vicente Casanova et al. "Networked Control Systems: control structures with bandwidth limitations". In: International Journal of Systems, Control and Communications 1.3 (2009), pp. 267-296. DOI: 10.1504/IJSCC. 2009 . 024556. eprint: https : / /www. inderscienceonline.com/doi / pdf/10.1504/IJSCC. 2009.024556 (cit. on p. 238).

[20] Tomás Lozano-Perez. Autonomous robot vehicles. Springer Science \& Business Media, 2012 (cit. on p. 238).

[21] Citlalli Gámez Serna and Yassine Ruichek. "Dynamic speed adaptation for path tracking based on curvature information and speed limits". In: Sensors 17.6 (2017), p. 1383 (cit. on p. 238).

[22] José E Naranjo et al. "Autonomous vehicle for surveillance missions in off-road environment". In: IEEE Intell. Veh. Symp. 2016, pp. 98-103 (cit. on p. 238).

[23] Victor Vaquero, Ely Repiso, and Alberto Sanfeliu. "Robust and realtime detection and tracking of moving objects with minimum 2D LIDAR information to advance autonomous cargo handling in ports". In: Sensors 19.1 (2019), p. 107 (cit. on p. 238).

[24] Jianyu Chen, Wei Zhan, and Masayoshi Tomizuka. "Autonomous Driving Motion Planning with Constrained Iterative LQR". In: IEEE Trans. Intell. Veh. (2019) (cit. on p. 238).

[25] Thomas Hellstrom and Ola Ringdahl. "Follow the Past: a path-tracking algorithm for autonomous vehicles". In: International journal of vehicle autonomous systems 4.2 (2006), pp. 216-224 (cit. on pp. 238, 251).

[26] Zirui Li et al. "Development and Evaluation of Two Learning-Based Personalized Driver Models for Pure Pursuit Path-Tracking Behaviors". In: 2018 IEEE Intelligent Vehicles Symposium (IV). June 2018, pp. 79-84. DOI: 10.1109/IVS.2018.8500618 (cit. on pp. 238, 251).

[27] Martin Lundgren. "Path tracking and obstacle avoidance for a miniature robot". In: Umeå University, Umeå, Master Thesis (2003) (cit. on pp. 238,251$)$. 
[28] Paolo Falcone et al. "A hierarchical model predictive control framework for autonomous ground vehicles". In: American Control Conf. 2008, pp. 37193724 (cit. on p. 238).

[29] Simon J Julier and Jeffrey K Uhlmann. "Unscented filtering and nonlinear estimation". In: Proc. IEEE 92.3 (2004), pp. 401-422 (cit. on pp. 238, 239).

[30] S. Haykin. Kalman filtering and neural networks. Wiley Online Library, 2001 (cit. on pp. 238, 239, 248).

[31] Greg Welch and Gary Bishop. "An introduction to the Kalman filter". In: University of North Carolina: Chapel Hill, North Carolina, US 378 (2006) (cit. on pp. 238, 239, 248).

[32] Dan Simon. Optimal state estimation: Kalman, $H_{\infty}$, and nonlinear approaches. John Wiley \& Sons, 2006. ISBN: 9780470045336 (cit. on pp. 238, 248).

[33] Xian-Ming Zhang and Qing-Long Han. "Network-based $H_{\infty}$ filtering using a logic jumping-like trigger". In: Automatica 49.5 (2013), pp. 14281435. ISSN: 0005-1098. DOI: https://doi.org/10.1016/j.automatica. 2013.01.060 (cit. on p. 238).

[34] Yizhou Wang, Wenjie Chen, and Masayoshi Tomizuka. "Extended kalman filtering for robot joint angle estimation using mems inertial sensors". In: IFAC Proc. Vol. 46.5 (2013), pp. 406-413 (cit. on p. 239).

[35] Yun-Bo Zhao, Guo-Ping Liu, and David Rees. "Design of a Packet-Based Control Framework for Networked Control Systems". In: IEEE Transactions on Control Systems Technology 17.4 (July 2009), pp. 859-865. ISSN: 1063-6536. DOI: 10.1109/TCST. 2008. 2010946 (cit. on p. 239).

[36] Ángel. Cuenca et al. "A packet-based dual-rate PID control strategy for a slow-rate sensing Networked Control System". In: ISA Transactions 76 (2018), pp. 155-166. ISSN: 0019-0578. DOI: https://doi.org/10.1016/ j.isatra.2018.02.022 (cit. on p. 239).

[37] Julián Salt and Pedro Albertos. "Model-based multirate controllers design". In: IEEE Transactions on Control Systems Technology 13.6 (Nov. 2005), pp. 988-997. ISSN: 1063-6536. DOI: 10.1109/TCST . 2005.857410 (cit. on pp. 240, 253).

[38] Julián Salt et al. "A Multirate Control Strategy to the Slow Sensors Problem: An Interactive Simulation Tool for Controller Assisted Design". In: Sensors 14.3 (2014), pp. 4086-4110. ISSN: 1424-8220. DOI: 10.3390/ s140304086 (cit. on pp. 240, 242, 253). 
[39] Raul Mansano, Eduardo Godoy, and Arthur Porto. "The benefits of soft sensor and multi-rate control for the implementation of wireless networked control systems". In: Sensors 14.12 (2014), pp. 24441-24461 (cit. on p. 240).

[40] Mazayoshi Tomizuka. "Multi-rate control for motion control applications". In: 8th IEEE Int. Workshop on Advanced Motion Control. 2004, pp. 21-29 (cit. on p. 240).

[41] Jean-Chrysotome Bolot. "End-to-end packet delay and loss behavior in the Internet". In: ACM SIGCOMM Computer Communication Review. Vol. 23. 4. ACM. 1993, pp. 289-298 (cit. on p. 240).

[42] A. Cervin et al. "How does control timing affect performance? Analysis and simulation of timing using Jitterbug and TrueTime". In: IEEE Control Systems Magazine 23.3 (June 2003), pp. 16-30. ISSN: 1066-033X. DOI: 10.1109/MCS. 2003.1200240 (cit. on pp. 240, 255).

[43] T. Cooklev, J. C. Eidson, and A. Pakdaman. "An Implementation of IEEE 1588 Over IEEE 802.11b for Synchronization of Wireless Local Area Network Nodes". In: IEEE Transactions on Instrumentation and Measurement 56.5 (Oct. 2007), pp. 1632-1639. ISSN: 0018-9456. DOI: 10. 1109/TIM. 2007.903640 (cit. on p. 243).

[44] T. Fukao, H. Nakagawa, and N. Adachi. "Adaptive tracking control of a nonholonomic mobile robot". In: IEEE Trans. Robot. Autom. 16.5 (2000), pp. 609-615 (cit. on p. 246).

[45] P. Khargonekar, K. Poolla, and A. Tannenbaum. "Robust control of linear time-invariant plants using periodic compensation". In: IEEE Transactions on Automatic Control 30.11 (Nov. 1985), pp. 1088-1096. ISSN: 0018-9286. DOI: 10.1109/TAC. 1985.1103841 (cit. on p. 248).

[46] Ángel Cuenca et al. "A non-uniform multi-rate control strategy for a Markov chain-driven Networked Control System". In: Information Sciences 321 (2015). Security and privacy information technologies and applications for wireless pervasive computing environments, pp. 31-47. ISSN: 0020-0255. DOI: https://doi.org/10.1016/j.ins.2015.05.035 (cit. on p. 254).

[47] Y. Tipsuwan and Mo-Yuen Chow. "Gain scheduler middleware: a methodology to enable existing controllers for networked control and teleoperation - part I: networked control". In: IEEE Transactions on Industrial Electronics 51.6 (Dec. 2004), pp. 1218-1227. ISSN: 0278-0046. DOI: 10.1109/TIE. 2004.837866 (cit. on p. 255).

[48] Katsuhiko Ogata. Discrete-time control systems. Vol. 2. Prentice Hall Englewood Cliffs, NJ, 1995 (cit. on p. 255). 
[49] Karl J Aström and Tore Hägglund. "PID controllers: theory, design, and tuning". In: Instrument Society of America 67 (1995) (cit. on p. 255). 


\section{Chapter 9}

\section{Conclusions}

This chapter concludes the work done throughout this doctoral work. From the NCS framework, event-triggered and packet-based control systems have been studied using both theoretical and practical points of view. Additionally, multi-rate and predictor-based control techniques have been used to deal with some kind of typical problems that appear in NCS.

In this conclusion section, the main contributions and future works are presented. Seven papers have been published from the work developed in the thesis. The impact factor of these papers is analyzed, and a list of co-authors is shown.

\subsection{Main contribution}

This doctoral work has completely achieved the goals initially proposed. Eventbased control, packet-based control, multi-rate control and predictor-based control techniques have been integrated to deal with certain problems that usually arise in NCS, such as, time-varying delays, packet dropouts, and packet disorder. In addition, by means of this control technique integration, the use of NCS resources has been reduced while maintaining satisfactory control performance.

Control system stability has been analyzed in terms of LMIs, and also by the QFT tool from the frequency point of view.

The benefits provided by the developed designs have been tested at the simulation level using a self-made simulator and Truetime, both of them based on Matlab/Simulink. In addition, they have been experimentally validated through several platforms such as an inverted pendulum, a portico robotic structure, and low-cost mobile robots. 
What follows is a summary of the contributions of each of the articles:

- Paper 1 deals with the real implementation of a periodic event-triggered control in a Networked Control System. The bandwidth used by the control loop in the shared communication medium is reduced by using a threshold-based communication.

- Paper 2 faces time-varying network-induced delays, packet dropouts and packet disorder in a Networked Control System by means of a delaydependent packet-based dual-rate control strategy. Slow-rate sensing enables to achieve energy saving and to avoid packet disorder. Fast-rate actuation makes reaching the desired control performance possible. The proposed control solution is able to approximately reach the nominal (no-delay, no-dropout) performance despite the existence of time-varying delays and packet dropouts. Control system stability is ensured in terms of probabilistic Linear Matrix Inequalities (LMIs).

- Paper 3 introduces a new procedure in order to properly analyze SISO dual-rate systems (DRS) and provides straightforward answers to some common general questions about this kind of systems. Frequency response analysis based on DRS lifting modeling can lead to interesting results about stability margins or performance prediction. As a novelty, it is explained how to understand DRS frequency response and how to handle it for an easy computation of magnitude and phase margins keeping classical frequency domain methods.

- In paper 4, a novel delay-independent packet-based dual-rate control structure for a networked control system is proposed. This work is a better variation of the solution presented in paper 2 . As a delay-independent control solution, no network-induced delay measurement is needed for controller implementation. In addition, the control scheme is applicable to open-loop unstable plants. As in paper 2, control system stability is ensured in terms of LMI and simulation results are experimentally validated.

- Paper 5 analyzes and compares dual-rate inferential control and modelbased dual-rate control trying to assess their behavior under different perspectives. The algorithm explained in paper 3 is used for computing a MR system frequency response for these control structures. The robust performance and disturbance effects are studied in detail under sample losses and process uncertainty and some considerations are reported. A 
new QFT (quantitative feedback theory) procedure for dual-rate systems analysis is also described.

- In paper 6, a novel control approach integrates solutions from papers 1, 2 and 4 and a time-varying dual-rate Kalman filter. The systematic combination of these control techniques allows a UGV to track the desired path preserving performance properties, despite:

- existing scarce data due to the reduced usage of the wireless sensor device, which results in less number of transmissions through the network, and hence, bandwidth and battery saving,

- appearing some wireless communication problems such as time-varying delays, packet dropouts and packet disorder, and

- coping with a realistic scenario where external disturbance and sensor noise can arise.

- Paper 7 presents a novel remote control solution for an Autonomous Vehicle, where the system structure is split into two sides through a communication network, which introduces time-varying delays and packet disorder. An extended Kalman filter is used to cope with the non-linearities that appear in the global model of the AV. The EKF fuses the data provided by the sensing devices of the AV in order to estimate the AV state, reducing the noise effect. Additionally, the EKF includes an $h$-step-ahead state prediction stage, which, together with the consideration of a packet-based control strategy, enables facing the network-induced delays. Since the AV position is provided by a camera, which is a slow sensing device, a dualrate controller is required to achieve certain desired (nominal) dynamic control performance. The use of a dual-rate control framework additionally enables saving network bandwidth and deals with packet disorder.

\subsection{Future work}

The following section proposes three different suggestions for future works using the results presented in the three parts of the thesis.

- Regarding event-triggered control (Paper 1), a detailed analysis of the results when using threshold-based communication shows that the need for bandwidth is not constant during the life of the system. It depends on the state of the controlled plant which in turn depends on the reference signal 
and on the presence of significant disturbances. The pre-arranged values for the thresholds have been obtained in certain environment conditions (reference signal to be followed, communication delays, signals noise, performance index, etc). If these conditions change, maybe the pre-arranged values are not the most appropriate. This suggests an adaptive, variable threshold-based communication in which the values for the thresholds are dynamically modified depending on the plant state. This adaptive policy is expected to improve the results obtained.

In addition, when using constant thresholds the system does not reach an equilibrium position. A certain ripple around the desired value appears when the reference signal is constant. The presence of this undesired characteristic is difficult to be seen in the plant used in this thesis because the upwards position of the inverted pendulum is an unstable equilibrium point and the same ripple is always present, even when conventional control is applied. Using a stable plant the ripple caused by the threshold can be considered in the performance index as a new factor to measure the system behavior. Variable thresholds are expected to help in solving this problem without increasing the consumed bandwidth.

Furthermore, some parts of the control system, such as an integral action, accumulate control action even when the signals haven't been sent. This produces a ripple in the control of \pm Control threshold which leads to a worsening of the ripple in the system output. A possible improvement could involve studying what to do with these parts of the control algorithm when the threshold isn't overcome.

Another improvement to be considered is to use a different sample period in the threshold comparison, leading to a dual-rate structure that could improve the performance without increasing the consumed bandwidth.

- The sections on packet-based and multi-rate control solves problems pertaining to NCS. Paper 7 also presents a non-linear model using these solutions for an autonomous vehicle that, due to the future information obtained through these techniques, can remotely perform high-level tasks such as decision making and variable monitoring. This final paper is clearly focused towards the application of these strategies to autonomous driving systems. The paper briefly describes trajectory decision-making strategies, which can be developed and therefore improved in future works. 
- The third section presents a method for obtaining and analyzing the frequency response of SISO dual-rate systems. It also studies their behavior when faced with uncertainties or network problems using QFT procedures. The examples depicting these techniques are inferential control compared to multirate control. A similar QFT study for the cases of packet-based or event-triggered control presented in this thesis could stem from this research, as their stability has been analyzed through LMIs.

\subsection{Impact factor report}

The first paper, "Control of the rotary inverted pendulum through thresholdbased communication"; the second paper, "A packet-based dual-rate PID control strategy for a slow-rate sensing networked control system" and the fifth paper, "Multirate Control Strategies for avoiding samples losses. Application to UGV path tracking" have been published in ISA Transactions. The first page of these articles are seen in figures 9.1, 9.2 and 9.5, respectively.

ISA Transactions is in the first quartile from 2018 Journal Citation Reports with an Impact Factor of 4.343 and a 5-year Impact Factor of 4.472. The journal is classified in 14th place out of 62 in the field of automation \& control systems, 8 th out of 88 in the field of engineering, multidisciplinary and 6 th out of 61 in the filed of instruments \& instrumentation.

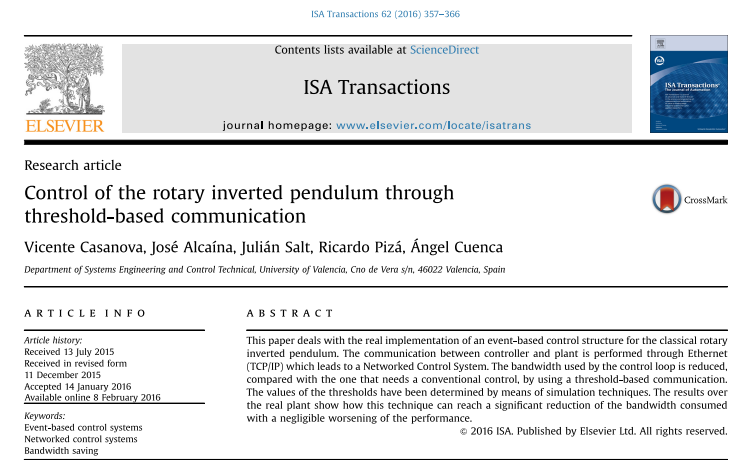

Figure 9.1: First page of paper 1 published in ISA Transactions

The third paper, "Dual-Rate Sampled-Data Systems Frequency Response: Interpretation and Some interesting consequences" has been published in the International Journal of General Systems and the first page of the article is seen in Figure 9.3. 


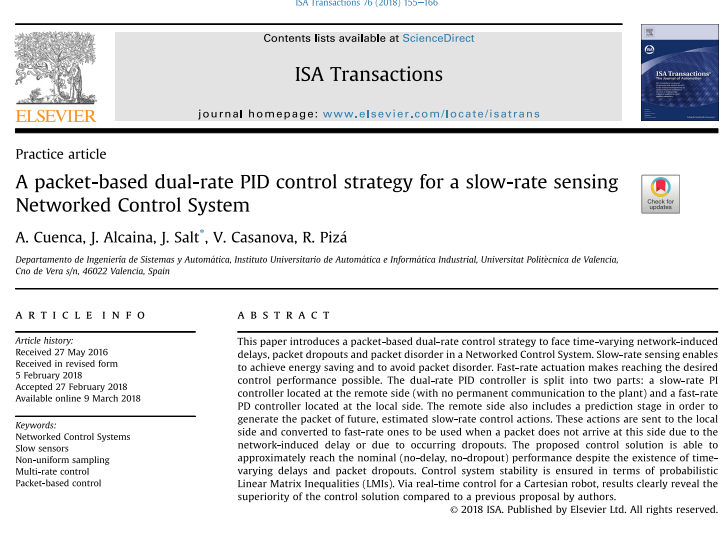

Figure 9.2: First page of paper 2 published in ISA Transactions

International Journal of General Systems is in the second quartile from 2018 Journal Citation Reports with an Impact Factor of 2.259 and a 5-year Impact Factor of 2.169. The journal is classified in 33d place out of 105 in the field of computer science, theory \& methods.

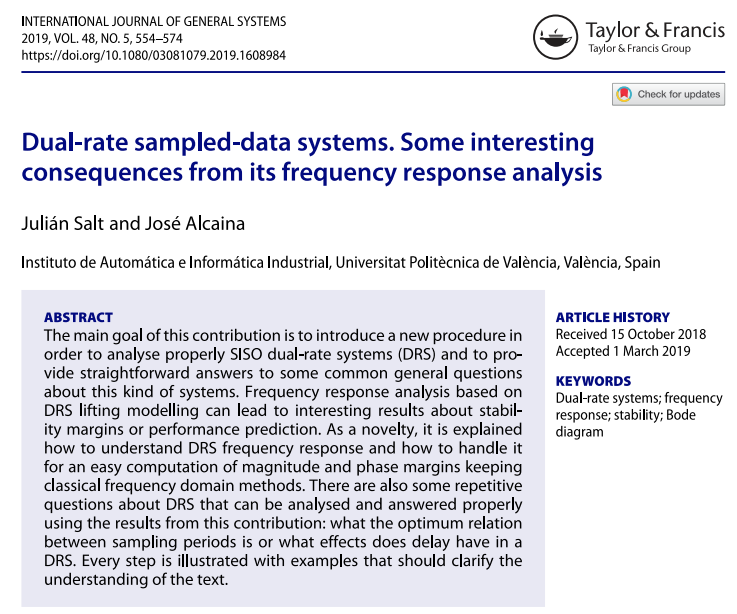

Figure 9.3: First page of paper 3 published in International Journal of General Systems

The fourth paper, "Delay-independent dual-rate PID controller for a packetbased networked control system" has been published in Information Sciences and the first page of the article is seen in Figure 9.4. 
Information Sciences is in the first quartile from 2018 Journal Citation Reports with an Impact Factor of 5.524 and a 5-year Impact Factor of 5.305. The journal is classified in 9th place out of 155 in the field of computer science, information systems.

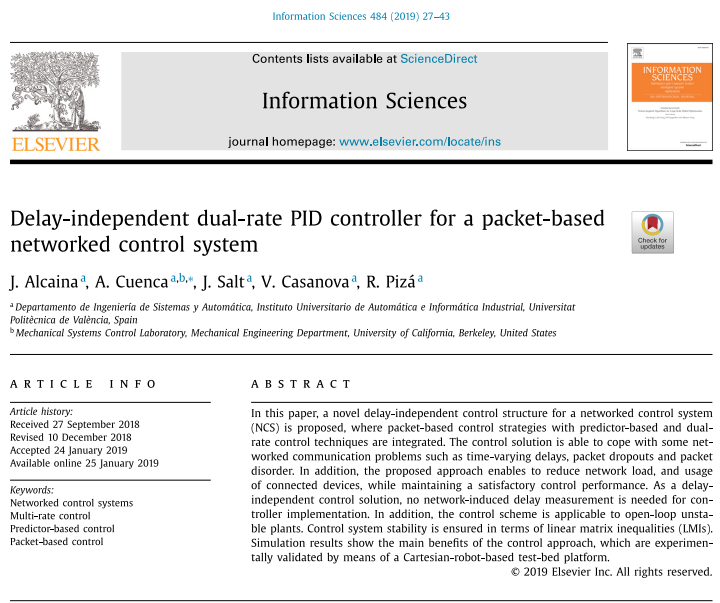

Figure 9.4: First page of paper 4 published in Information Sciences

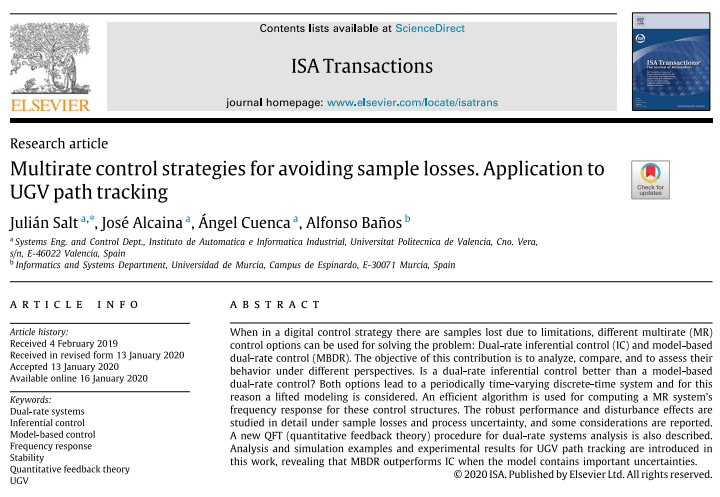

Figure 9.5: First page of paper 5 published in ISA Transactions

The sixth paper, "Energy-efficient Control for an Unmanned Ground Vehicle in a Wireless Sensor Network" has been published in Journal of Sensors and the first page of the article is seen in Figure 9.6.

Journal of Sensors is in the second quartile from 2018 Journal Citation Reports with an Impact Factor of 2.024 and a 5-year Impact Factor of 2.090. 
The journal is classified in 140th place out of 266 in the field of engineering, electrical \& electronic and in 30th place out of 61 in the field of instruments $\&$ instrumentation.

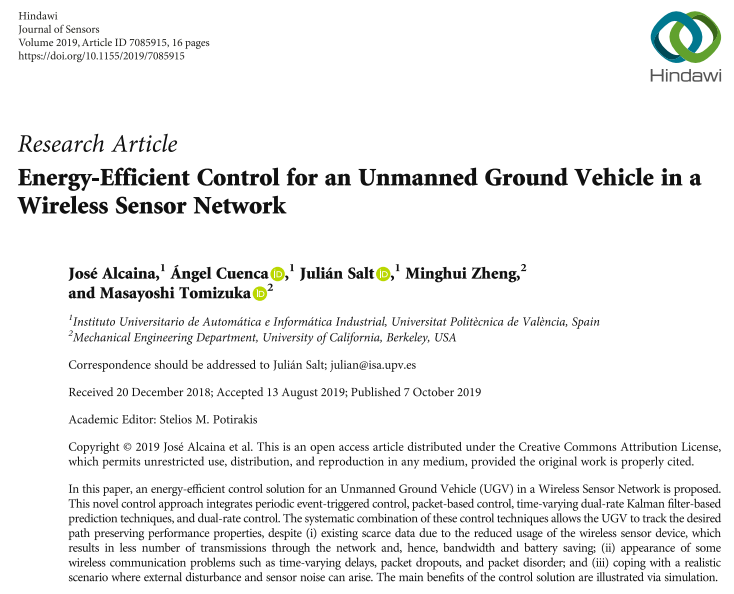

Figure 9.6: First page of paper 6 published in Journal of Sensors

The seventh paper, "A Remote Control Strategy for an Autonomous Vehicle with Slow Sensor using Kalman Filtering and Dual-rate Control" has been published in Sensors and the first page of the article is seen in Figure 9.7.

Sensors is in the first quartile from 2018 Journal Citation Reports with an Impact Factor of 3.031 and a 5-year Impact Factor of 3.302. The journal is classified in 23rd place out of 84 in the field of chemistry, analytical, in 12th place out of 26 in the field of electrochemistry, and in 15th out of 61 in the field of instruments \& instrumentation.

These impact indices and others are summarized in Table 9.1. Journal Impact Factor (JIF), 5-year JIF, Immediacy index, best JIF considering all categories and the corresponding Q quartile are all obtained from Journal Citation Reports. From Journal Metrics of Scopus the SCImago Journal Rank (SJR) and the Source Normalized Impact for Paper (SNIP) are taken. Finally, H5-index and H5-median are obtained from Google Scholar Metrics. 
Article

A Remote Control Strategy for an Autonomous

Vehicle with Slow Sensor Using Kalman Filtering and Dual-Rate Control

Ángel Cuenca ${ }^{1, *} \odot$, Wei Zhan ${ }^{2}$, Julián Salt ${ }^{1} \odot$, José Alcaina ${ }^{1}$, Chen Tang ${ }^{2}$ and Masayoshi Tomizuka

Instituto Universitario de Automática e Informática Industrial, Universitat Politècnica de València,

46022 València, Spain

Mechanical Engineering Department, University of California, Berkeley, CA 94720, USA

acuencaeasa.upves

Received: 24 May 2019; Accepted: 4 July 2019; Published: 6 July 2019

Abstract: This work presents a novel remote control solution for an Autonomous Vehicle (AV) where the system structure is split into two sides. Both sides are assumed to be synchronized and linked through a communication network, which introduces time-varying delays and packet disorder An Extended Kalman Filter (EKF) is used to cope with the non-linearities that appear in the global model of the AV. The EKF fuses the data provided by the sensing devices of the AV in order to estimate the AV state, reducing the noise effect. Additionally, the EKF includes an $h$-step-ahead state prediction stage, which, together with the consideration of a packet-based control strategy. enables facing the network-induced delays. Since the AV position is provided by a camera, which is a slow sensing device, a dual-rate controler is required to achieve certain desired (nominal) dynamic bandwidth and deals with packet disorder As the peth-traking controt alborithmy pure pursut is used. Application results show that despite existing measurements, the $\mathrm{AV}$ is able to track the desired path keeping the nominal control performance.

Keywords: autonomous vehicle; slow sensor; Kalman filter; networked control; dual-rate control

Figure 9.7: First page of paper 7 published in Sensors 


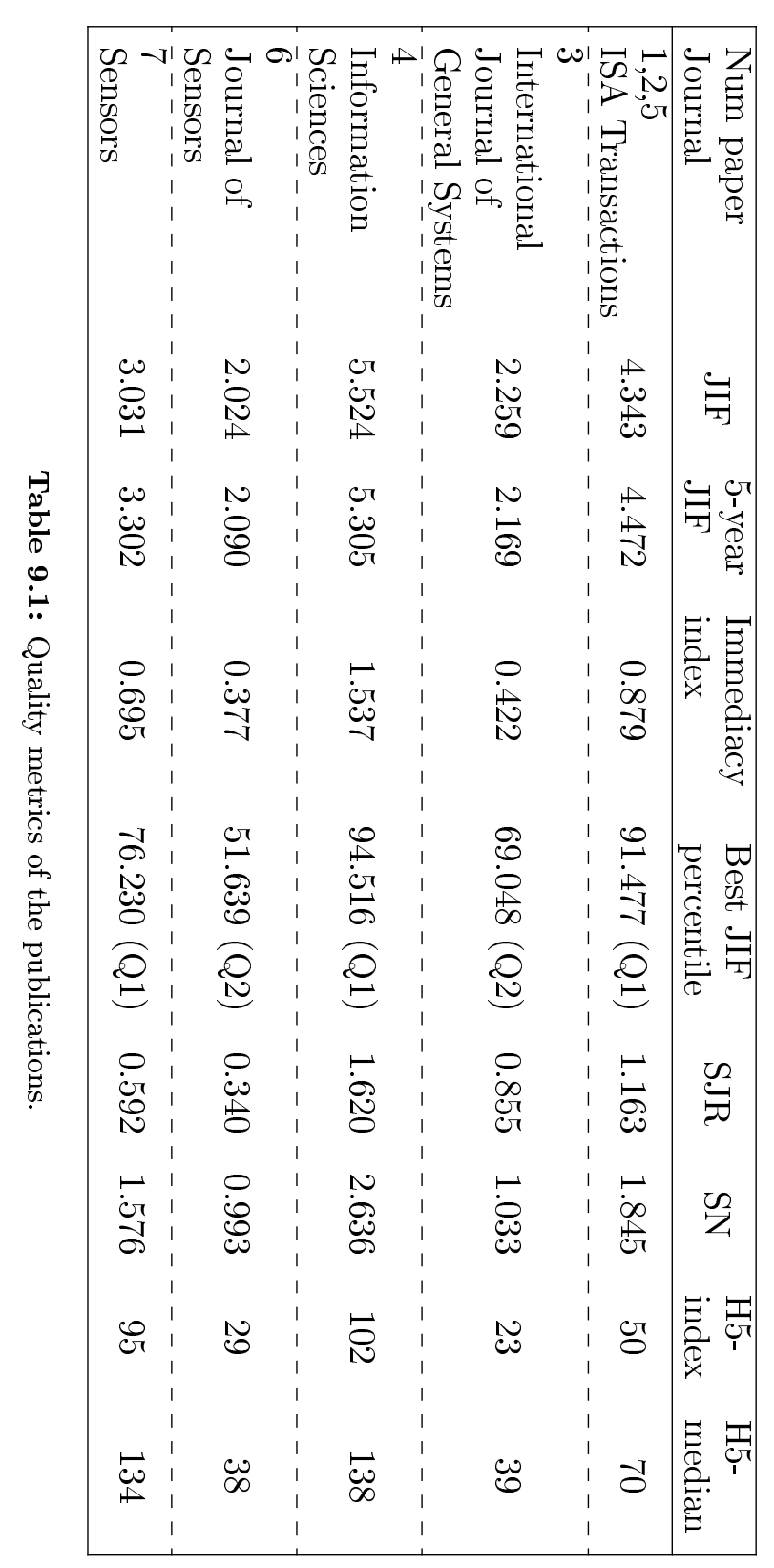




\subsection{Coauthors affiliation}

Ángel Cuenca

Instituto Universitario de Automática e Informática Industrial Universitat Politècnica de València

acuenca@isa.upv.es

Papers 1, 2, 4, 5, 6, 7

Julián Salt

Instituto Universitario de Automática e Informática Industrial Universitat Politècnica de València

julian@isa.upv.es

Papers 1, 2, 3, 4, 5, 6, 7

Vicente Casanova

Instituto Universitario de Automática e Informática Industrial Universitat Politècnica de València

vcasanov@isa.upv.es

Papers 1, 2, 4

Ricardo Pizá

Instituto Universitario de Automática e Informática Industrial Universitat Politècnica de València

rpiza@isa.upv.es

Papers 1, 2, 4

Alfonso Baños

Informatics and Systems Department

Universidad de Murcia, Campus de Espinardo

abanos@um.es

Paper 5

Minghui Zheng

Mechanical Engineering Department

University of California, Berkeley

minghuizheng@berkeley.edu 
Paper 6

Masayoshi Tomizuka

Mechanical Engineering Department

University of California, Berkeley

tomizuka@berkeley.edu

Papers 6, 7

Wei Zhan

Mechanical Engineering Department

University of California, Berkeley

wzhan@berkeley.edu

Paper 7

Chen Tang

Mechanical Engineering Department

University of California, Berkeley

chen_tang@berkeley.edu

Paper 7 